\title{
The molecular mechanisms of myelin disassembly
}

\author{
Dissertation \\ for the award of the degree \\ "Doctor rerum naturalium" (Dr.rer.nat.) \\ of the Georg August University Göttingen, Faculty of Biology \\ within the doctoral program \\ "Molecular Biology of Cells"
}

submitted by

Marie-Theres Weil

from

Mainz, Germany

Göttingen,

10th February 2016 


\section{Examination board}

Prof. Dr. Mikael Simons (Reviewer)

Research Group of Cellular Neuroscience

Max Planck Institute for Experimental Medicine

Prof. Dr. Alexander Flügel (Reviewer)

Department of Neuroimmunology

Institute for Multiple Sclerosis Research

University Medical Center Göttingen

Prof. Dr. Holger Reichardt

Department of Cellular and Molecular Immunology

University Medical Center Göttingen

Prof. Dr. Klaus-Armin Nave

Department of Neurogenetics

Max Planck Institute for Experimental Medicine

Prof. Dr. Dr. Hannelore Ehrenreich

Clinical Neuroscience

Max Planck Institute for Experimental Medicine

Prof. Dr. med. Wolfgang Brück

Institute of Neuropathology

University Medical Center Göttingen

Date of oral examination:

28.04.2016 


\section{Affidavit}

I hereby declare that the $\mathrm{PhD}$ thesis entitled "The molecular mechanisms of myelin disassembly" has been written independently by myself with no other contributory sources and aids than quoted.

Göttingen, 10th February 2016

Marie-Theres Weil 

Für Yasmin. 



\section{Acknowledgement}

Never blame anyone in your life. Good people give you happiness. Bad people give you experience. Worst people give you a lesson \& Best people give you memories.

First of all, I would like to thank my supervisor Prof. Mikael Simons for allowing me to conduct my research in his laboratory. His scientific way of thinking was an inspiration and provided important guidance for the project. I would also like to thank him for the scientific discussions during my $\mathrm{PhD}$ project and teaching my a lot about science apart from experiments.

I want to thank my thesis committee members Prof. Dr. Alexander Flügel and Prof. Dr. Holger Reichardt for their interest in my project and their contribution during my progress reports. Furthermore, I would like to acknowledge Prof. Dr. Alexander Flügel for agreeing to act as a second reviewer. I also thank Prof. Hannelore Ehrenreich, Prof. Wolfang Brück and Prof. Klaus-Armin Nave for being part of my examination board.

I thank my collaborators, Dr. Steffen Frey, Prof. Petri Kursula, Dr. Anne Winkler, Dr. Claudia Wrzos, Prof. Christine Stadelmann-Nessler, Elisa Romanelli, Prof. Martin Kerschensteiner, David Enz, Prof. Nicole SchaerenWiemers for their supportive contribution towards my project.

I would like to highlight how much I appreciate the invaluable help of the EM facility with Wiebke, Torben, Martin, Boguscha and Christian that I will always remember as my happy place in the MPI. A special thank you to Wiebke and Torben not only for the awesome scientific support, but also for the emotional support. I will never forget the funny freezing days, the support with coffee and chocolate and all the laughs that we shared! You prove that one can do good science and most importantly, have fun doing it and stay true to yourself.

Many thanks to my colleagues Basti, Natalia, Mosi, Aniket, Schanila, Nils, 
Shweta, Nic, Lena, Dirk, Ludo, Caro, Tina, Shima, Maryam, Paula, Minhui, Uli and Sebastian for the nice working atmosphere. Giselheid - Danke für die nette Atmosphere in der Zellkultur und die tollen Gespräche, wobei die tollen Zellen immer Nebensache schienen.

Karla - Danke für deine oft unsichtbare Unterstützung im Labor. Man merkt immer wie dankbar man für jemand sein sollte, wenn die Person im Urlaub ist.

Especially, I would like to thank Basti for helping me to get started in the laboratory and for the enjoyable scientific guidance. Without you, I would not have survived my first year of PhD! Furthermore, I want to acknowledge Ludo's crucial scientific support during coffee breaks and in the laboratory. I will always remember the strong coffee, the good conversations and the funny moments we shared.

Moreover, I want to thank all my friends for the great time we spent together. You made life in Göttingen and the MPI so much for fun and memorable that we could also enjoy very though times together. Without the fun coffee breaks planning our free time, the late night dinners in and outside the MPI, beers and cocktail sessions, life would have been much more harder. The memories from this time will always be very special to me.

Franz, I am grateful for your constant belief in me and for providing invaluable support even in very tough times.

Foremost, I am very blessed with the constant support of my parents Christian and Cornelia and my sister Yasmin. Your constant understanding and persistent love was crucial to persevere. Thank you for always encouraging me to follow my beliefs and my morals. Without your belief in me, it would have been possible! 


\section{Contents}

List of publications $\quad$ xviii

Abstract $\quad$ xix

1 Introduction 1

1.1 Process of myelination . . . . . . . . . . . . 2

1.2 Myelin structure . . . . . . . . . . . . . . . 3

1.3 Myelin function . . . . . . . . . . . . . . . 5

1.4 Molecular composition of myelin . . . . . . . . . 5

1.4.1 Lipid composition of myelin . . . . . . . . . . . 5

1.4.2 Protein composition of myelin . . . . . . . . . . . 9

1.5 Demyelinating diseases . . . . . . . . . . . . . . . . 19

1.5.1 Multiple sclerosis . . . . . . . . . . . . . . . . 19

1.5.2 Neuromyelitis optica . . . . . . . . . . . . . 27

1.6 Project aim . . . . . . . . . . . . . . . . 31

2 Material and Methods 33

2.1 Material . . . . . . . . . . . . . . . . . . . 33

2.1.1 Chemicals and consumables . . . . . . . . . . 33

2.1.2 Antibodies used in this thesis . . . . . . . . . . 33

2.1 .3 Kits used . . . . . . . . . . . . . . . . 34

2.1.4 Enzymes used in this studies . . . . . . . . . . 35

2.1.5 General Buffers and Solutions . . . . . . . . . . 35

2.1 .6 Primer . . . . . . . . . . . . . . . 39

2.1 .7 Software . . . . . . . . . . . . . . 39

2.2 Methods . . . . . . . . . . . . . . . . . 40

2.2 .1 Cell culture . . . . . . . . . . . . . . . 40

2.2 .2 In vitro assays . . . . . . . . . . . . . . . 42

2.2.3 Perfusion and Immunohistochemistry . . . . . . . . 46

2.2.4 Molecular Biology . . . . . . . . . . . . . . . 47

2.2 .5 Biochemistry . . . . . . . . . . . . . . . 49

2.2 .6 Biomimetic assays . . . . . . . . . . . . . 52

2.2 .7 Spectroscopy . . . . . . . . . . . 56 
2.2 .8 Animal studies . . . . . . . . . . . . . . . 56

2.2 .9 Electron microscopy . . . . . . . . . . . . . 58

2.2.10 Image Processing and Statistical Analysis . . . . . . . 61

3 Results $\quad 62$

3.1 Myelin pathology in demyelinating diseases . . . . . . . . . 62

3.1.1 Ultrastructural characterization of myelin pathology in early NMO lesions . . . . . . . . . . . . . . . 62

3.1.2 Loss of MAG staining in focal NMO lesions . . . . . 65

3.1.3 Myelin pathology in Lewis rats injected with MOG antibody ................. 66

3.1.4 Myelin degradation in Biozzi experimental autoimmune encephalomyelitis . . . . . . . . . . 71

3.1.5 Loss of MBP from vesiculated myelin in early NMO lesions . . . . . . . . . . . . . . . 73

3.2 Reverse phase transition of MBP . . . . . . . . . 74

3.2.1 Liposomes binding assay with $\mathrm{MBP} \mathrm{F} \rightarrow \mathrm{S}$ mutants . . 76

3.2.2 Spectroscopy of WT peptides of amphipathic helices . 78

3.2.3 Lipid droplet assay using MBP F $\rightarrow$ S mutants . . . . 79

3.2.4 SLB-MBP GUV assay with MBP F $\rightarrow$ S mutants . . . 80

3.2.5 Transfection of PtK2 cells with MBP F $\rightarrow$ S mutants . 81

3.2.6 Transfection of primary mouse oligodendrocytes with MBP F $\rightarrow$ S mutants . . . . . . . . . . . . . 82

3.2.7 Tools to detect reverse phase transition . . . . . . 84

3.3 In vitro reverse phase transition model . . . . . . . . . . . 87

3.3.1 Disruption of electrostatic interaction between MBP and the membrane . . . . . . . . . 87

3.3.2 Disruption of hydrophobic interaction between MBP and the membrane . . . . . . . . . . . . 90

3.4 Characterization of reverse phase transition in vitro . . . . . 92

3.4.1 Detachment of MBP from the membrane upon treatment with antibodies against $\mathrm{O} 1$ and $\mathrm{O} 4 \ldots . . .95$

3.4.2 Self-interaction of MBP upon O1 and O4 treatment . 97

3.4.3 Compaction and extrusion of MBP upon $\mathrm{O} 1$ and $\mathrm{O} 4$ treatment or primary oligodendrocytes . . . . . . . . 100

3.5 Characterization of reverse phase transition of MBP in the cuprizone model in vivo . . . . . . . . . . . . . . . . . 101

3.5.1 QD9 immunohistochemistry of cuprizone time course . 101

3.5.2 Early loss of MAG staining in cuprizone treated mice 104

3.5.3 Myelin pathology in cuprizone time course . . . . . . . 104

3.5.4 QD9 histology in early NMO lesions . . . . . . . . 106

3.6 QD9 staining in lesions of Multiple sclerosis patients . . . . 108

3.7 Calcium as underlying cause for reverse phase transition of MBP ......................... 109 
3.7.1 Treatment of acute brain slices with Ionomycin . . . . 109

3.7.2 Treatment of ex vivo brain slices with EGTA after AQP4 antibody injection in Lewis rats . . . . . . . 111

4 Discussion

4.1 Myelin pathology in demyelinating diseases . . . . . . . . . 114

4.1.1 Myelin pathology in oligodendropathies . . . . . . . . 114

4.1.2 Immune-mediated myelin pathology . . . . . . . . . . 116

4.1.3 Mechanism of myelin fragmentation . . . . . . . . . 117

4.1.4 Inner tongue as initiation site for myelin pathology . . 120

4.2 Causes of Myelin Degradation . . . . . . . . . . . . . . . . 121

4.3 Downstream effects of calcium infl ux . . . . . . . . . . 122

4.4 Reverse phase transition of MBP . . . . . . . . . . . . . 124

$\begin{array}{lll}5 & \text { Summary } & 127\end{array}$

$\begin{array}{lr}\text { Appendix } & 129\end{array}$

$\begin{array}{ll}\text { Bibliography } & 136\end{array}$

$\begin{array}{ll}\text { Curriculum vitae } & 161\end{array}$ 



\section{List of Figures}

1.1 Myelin structure and protein distribution at the axon-glia junction in the CNS ................ 4

1.2 Overview of the most abundant proteins and lipids and their subcellular localisation in myelin . . . . . . . . . 6

1.3 Simplified overview of the post-translational modifications and their implication on the localisation and function of MBP 13

1.4 Electron microscopy-based reconstruction of $18.5 \mathrm{kDa} \mathrm{MBP}$ from bovine brain reconstituted with gangliosides . . . . . 16

1.5 History of Multiple sclerosis . . . . . . . . . . . . 20

1.6 Molecular and cellular changes upon cuprizone treatment . . 25

1.7 Induction and disease course of experimental autoimmune encephalomyelitis . . . . . . . . . . . . 27

1.8 Suggested mechanism for Neuromyelitis optica pathology . . 30

3.1 Myelin pathology in early NMO lesions . . . . . . . . . 65

3.2 Early NMO lesions are devoid of MAG staining . . . . . . . 67

3.3 Early loss of MAG staining prior to MBP loss in human NMO lesions . . . . . . . . . . . . . . . . 6 68

3.4 Early lesions of MOG antibody injection display enlarged inner tongue and vesiculation of inner myelin layers . . . . . . 70

3.5 Biozzi EAE lesions during first relapse show signs of myelin pathology with enlarged inner tongue and vesiculation of the innermost layers of myelin . . . . . . . . . . . . 72

3.6 Vesicules of inner myelin layers in early NMO lesions consist of myelin membrane despite lack MBP . . . . . . . . . . . 74

3.7 Reverse phase transition of MBP . . . . . . . . . 75

3.8 All $\mathrm{F} \rightarrow \mathrm{S}$ mutants are capable of binding the membrane and crosslinking liposomes . . . . . . . . . . . . . 77

3.9 Phenylalanines mediate structural change of MBP peptides . 79

3.10 Mutations of four phenylalanines abolishes MBP droplet formation . . . . . . . . . . . . . . 80

3.11 Four phenylalanines in MBP are necessary to mediate GUV bursting . . . . . . . . . . . . . . 81 
3.12 Four phenylalanines in MBP mediate domain formation in PtK2 cells . . . . . . . . . . . . . . . 82

3.13 Primary mouse oligodendrocytes as in vitro model system . . 83

3.14 Four phenylalanines are necessary for the integration of MBP in sheets of oligodendrocytes . . . . . . . . . 84

3.15 QD9 recognizes full length monomeric MBP . . . . . . . . 86

3.16 QD9 recognizes structurally aberrant $\mathrm{MBP}$ like $\mathrm{F} \rightarrow \mathrm{S} \mathrm{MBP}$ mutant . . . . . . . . . . . . . . 87

3.17 Ionomycin detaches MBP from the membrane and leads to decompaction . . . . . . . . . . . . . . 89

3.18 Sphingosine treatment of oligodendrocytes lead to QD9 unmasking . . . . . . . . . . . . . . 90

3.19 Fumonisin B1 induces QD9 unmasking and decompaction in primary mouse oligodendrocytes . . . . . . . . . . 91

3.20 Methyl- $\beta$-cyclodextrin increases QD9 staining in the sheets of mouse oligodendrocytes . . . . . . . . . . . . . . 92

3.21 Clustering of myelin surface epitopes lead to rapid QD9 unmasking in the absence of complement . . . . . . . . . . . 94

3.22 Treatment with myelin surface antibodies leads to MBP detachment from membrane . . . . . . . . . . . . 96

$3.23 \mathrm{O} 1$ and $\mathrm{O} 4$ treatment of cells increases the CHAPS-solubility

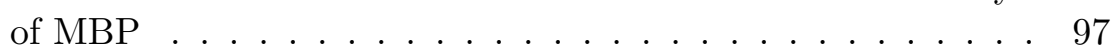

3.24 Antibody-mediated FRET assay indicates loss of self-interaction upon detachment of MBP from the membrane . . . . . . . . 99

3.25 Decompaction of primary mouse oligodendrocytes upon myelin surface antibody treatment . . . . . . . . . . . 101

3.26 QD9 unmasking in early stages of cuprizone time course . . . 103

3.27 Loss of MAG staining upon short cuprizone treatment . . . . 104

3.28 Ultrastructural changes in myelin upon cuprizone treatment . 106

3.29 Early NMO lesions in Lewis rats display QD9 unmasking . . 107

3.30 QD9 stains border zone around chronic white matter lesions of Multiple Sclerosis patients . . . . . . . . . . . 108

3.31 Vesiculation of the innermost myelin sheath upon Ionomycin treatment of acute brain slices . . . . . . . . . . . . . . 110

3.32 EGTA treatment of acute brain slices after AQP4 antibody injection prevents QD9 unmasking . . . . . . . . . 112

4.1 Myelin fragmentation in demyelination . . . . . . . . . 118

4.2 Microglia engulf QD9 positive material and are in association with QD9 positive fibers in the corpus callosum of cuprizone treated mice . . . . . . . . . . . . . . . . . 119

4.3 Cytoplasmic domains of mature oligodendrocytes . . . . . . 121

4.4 Molecular disassembly of myelin . . . . . . . . . . . . . . 124 
5.1 Correlation of myelin ultrastructure with QD9 staining revealing different structural states of MBP under normal and pathological conditions . . . . . . . . . . . . . . 129

5.2 Acute axonal damage in focal NMO . . . . . . . . . . . . 131

5.3 Acute axonal damage in focal NMO . . . . . . . . . . . 131

5.4 Acute axonal damage in MOG-antibody induced lesions . . . 132

5.5 Acute axonal damage in EAE lesions . . . . . . . . . . . 132

5.6 Luxol Fast blue staining of cuprizone time course . . . . . . 134

5.7 Myelin pathology in Lysolecithin injections . . . . . . . . 135

5.8 Fragmentation of myelin in shiverer mice . . . . . . . 136 



\section{List of Tables}

2.1 Antibodies used in this thesis . . . . . . . . . . . . 34

2.2 Commercial kits . . . . . . . . . . . . . . 35

2.3 Enzymes used in this study . . . . . . . . . . . . . 35

2.4 Primers . . . . . . . . . . . . . . . . . . 39

2.5 Software used in this study . . . . . . . . . . . . . 40

2.6 Composition of SuperSATO medium . . . . . . . . . . . 41

2.7 Treatment of primary mouse oligodendrocytes . . . . . . . . 44

2.8 Settings on confocal microscope to measure FRET efficiency . 46

2.9 Cycling conditions for site-directed mutagenesis . . . . . . . 48

2.10 Cycling conditions for molecular cloning . . . . . . . . . 49

2.11 Composition of Tris-PAGE gels . . . . . . . . . . . 52

2.12 Lipids used for biomimetic studies . . . . . . . . . . 53

2.13 Liposome compositions used in this thesis . . . . . . . 53

2.14 GUV compositions used in this thesis . . . . . . . . 54

2.15 Automated freeze substitution protocol . . . . . . . . 60

2.16 Antibodies for Cryo-immunoelectron microscopy . . . . . . . 61

5.1 Generated plasmids . . . . . . . . . . . . . . . 133 



\section{List of abbreviations}

A

aa

$\mathrm{ABH}$

ACSF

APS

ATR-FT-IR spectroscopy

AQP4

BBB

$\mathrm{BME}$

bp

BSA

${ }^{\circ} \mathrm{C}$

CC

CD

CHAPS

Chol

Cit

CNP

CNS

CSF

$\mathrm{Cy}$

d

DAPI

$\mathrm{ddH}_{2} \mathrm{O}$

DDSA

DMEM

DMSO

dNTP

DPC

DTSSP

EAE
Ampere

Amino acid

Antibody high

Artifical cerebrospinal fluid

Ammonium persulfate

Attenuated Total Reflectance-

Fourier transformation infrared spectroscopy

Aquaporin 4

Blood brain barrier

Basal Medium Eagle

Base pair

Bovine serum albumin

Degree celsius

Corpus callosum

Circular dichroism

3-[(3-Cholamidopropyl)dimethylammonio]-1-

propanesulfonate

Cholesterol

Citrunille

2',3'-cyclic nucleotide 3'-phosphodiesterase

Central nervous system

Cerebrospinal fluid

Cyanine

Day

4'-6'-Diamidino-2-phenylindole

double distilled water

2-Dodecenylsuccinic acid anhydride

Dulbecco's modified eagle's medium

Dimethylsulfoxide

Deoxynucleotide triphosphate

Dodecyl phosphatidyl choline

$3,3^{6}$ dithiobis[sulfosuccinimidylpropionate]

Experimental autoimmune encephalomyelitis 


\begin{tabular}{|c|c|}
\hline EDTA & Ethylenediaminetetraacetic acid \\
\hline EAAT2 & Excitatory amino acid transporter \\
\hline EM & Electron microscopy \\
\hline Epon & Epoxy resin \\
\hline EPR & electron paramagnetic resonance spectroscopy \\
\hline ER & endoplasmatic reticulum \\
\hline FB1 & Fumonisin B1 \\
\hline FCS & Fetal calf serum \\
\hline FRET & Fluorescence resonance energy transfer \\
\hline $\mathrm{g}$ & Gram \\
\hline Galc & galactosylcerebroside \\
\hline GAPDH & Glyceraldehyde 3-phosphate dehydrogenase \\
\hline Golli & genes of the oligodendrocyte lineage \\
\hline GS & Goat serum \\
\hline Gua-HCl & Guanidinium-hydrochloride \\
\hline GUV & Giant unilamellar vesicle \\
\hline $\mathrm{h}$ & Hour \\
\hline HBSS & Hank's buffered saline \\
\hline HS & Horse serum \\
\hline ICC & Immunocytochemistry \\
\hline $\mathrm{IHC}$ & Immunohistochemistry \\
\hline ip & Intraperitoneal \\
\hline IPTG & Isopropyl $\beta$-D-1-thiogalactopyranoside \\
\hline ITO & Indium-tin oxide \\
\hline IUP & Intrinsically unstructured proteins \\
\hline $\mathrm{kD}$ & Kilodalton \\
\hline $\mathrm{KO}$ & Knock-out, constitutive mutant \\
\hline $\mathrm{Kr}$ & Krebs-Ringer-solution \\
\hline 1 & Liter \\
\hline LB & Luria broth \\
\hline LFB & Luxol Fast Blue \\
\hline LUV & large unilamellar vesicle \\
\hline $\mathrm{m}$ & Meter \\
\hline M & Molar \\
\hline MAG & Myelin associated glycoprotein \\
\hline MAPK & Mitogen-activated protein kinase \\
\hline MARCKS & Myristoylated alanine-rich C-kinase substrate \\
\hline MBP & Myelin basic protein \\
\hline $\mathrm{MCD}$ & Methyl- $\beta$-cyclodextrin \\
\hline MDL & Major dense line \\
\hline $\min$ & Minute \\
\hline MNA & Methylnadic anhydride \\
\hline MOG & Myelin oligodendrocyte glycoprotein \\
\hline MRI & Magnetic resonance imaging \\
\hline
\end{tabular}




\begin{tabular}{|c|c|}
\hline MS & Multiple Sclerosis \\
\hline MW & Molecular weight \\
\hline $\mathrm{n}$ & Number of samples \\
\hline NAWM & Normal appearing white matter \\
\hline NMO & Neuromyelitis optica \\
\hline NO & Nitric oxide \\
\hline n.s. & Non-significant \\
\hline OCD & oriented circular dichroism spectroscopy \\
\hline ODC & Oligodendrocyte \\
\hline OPC & Oligodendrocyte progenitor cell \\
\hline OSP & Oligodendrocytes-specific protein \\
\hline $\mathrm{p}$ & $\mathrm{P}$-value \\
\hline $\mathrm{P}$ & Postnatal day \\
\hline PAD & peptidylarginine deiminase \\
\hline PAGE & Polyacrylamide gel electrophoresis \\
\hline $\mathrm{PB}$ & Phosphate buffer \\
\hline PBS & Phosphate buffered saline \\
\hline $\mathrm{PC}$ & L- $\alpha$-phosphatidylcholine \\
\hline PCR & Polymerase chain reaction \\
\hline $\mathrm{PE}$ & L- $\alpha$-phosphatidylethanolamine \\
\hline PFA & Paraformaldeyhde \\
\hline PG & Dimyristoyl-sn-glcero-3-phosphoglycerol \\
\hline PIP2 & L- $\alpha$-phosphatidylinositol- 4,5 -bisphosphate \\
\hline PIP3 & L- $\alpha$-phosphatidylinositol-3,4,5-trisphosphate \\
\hline PLL & Poly-L-Lysine \\
\hline PLP & Proteolipid protein \\
\hline PNS & Peripheral nervous system \\
\hline PS & L- $\alpha$-lysophosphatidylserine \\
\hline PTEN & phosphatase and tensin homologue \\
\hline PTX & Pertussis toxin \\
\hline $\mathrm{rpm}$ & Rotations per minute \\
\hline RPMI & Roswell Park Memorial Institute Medium \\
\hline $\mathrm{RT}$ & Room temperature \\
\hline $\mathrm{SCH}$ & Spinal cord homogenate \\
\hline SD & Standard deviation \\
\hline SDS & Sodiumdodecylsulfate \\
\hline sec & Seconds \\
\hline SEM & Standard error of the mean \\
\hline SLB & supported lipid bilayer \\
\hline $\mathrm{SM}$ & Sphingomyelin \\
\hline SRCD & Synchrotron radiation circular dichroism spectroscopy \\
\hline SUV & Small unilamellar vesicle \\
\hline TB & Terrific broth \\
\hline TBS & TRIS-buffered saline \\
\hline
\end{tabular}




$\begin{array}{ll}\text { TEMED } & \text { Tetramethylenediamine } \\ \text { TFA } & \text { Trifluoroacetic acid } \\ \mathrm{T}_{\mathrm{m}} & \text { Melting temperature } \\ \text { Tris } & \text { 2-Amino-2-(hydroxymethyl)-1,3-propanediol } \\ \mathrm{U} & \text { Unit } \\ \mathrm{UV} & \text { Ultraviolet } \\ \mathrm{V} & \text { Volt } \\ \mathrm{WT} & \text { wild type } \\ \mathrm{w} / \mathrm{v} & \text { weight per volume }\end{array}$

DNA nucleotides purine or pyrimidine bases were coded as follows to describe DNA sequences:

A Adenine
C Cytosine
G Guanine
T Thymine 


\section{List of publications}

Weil MT., Möbius W., Winkler A., Ruhwedel T., Wrozs C., Raasakka A., Romanelli E., Bennett J., Enz L., Brück W., Nave KA., Kursula P., Kursula P., Kerschensteiner M., Schaeren-Wiemers N., Stadelmann-Nessler C., Simons M. (2016). Myelin basic protein network depolymerisation triggers vesicualr disruption of myelin sheath in demyelinating diseases, submitted

Romanelli E., Merkler D., Weil MT., Weber M., Nikic I., Potz S., Matznick F., Kreutzfeldt M., Ghanem A., Conzelmann K., Brück W., Routh M., Simons M., Bishop D., Misgeld T., Kerschensteiner M. (2016). Myelinosome formation represents an early stage of oligodendrocyte damage in multiple sclerosis and its animal model, under revision in Nature communications

Aggarwal S1., Snaidero N., Pähler G., Frey S., Sanchez P., Zweckstetter M., Janshoff A., Schneider A., Weil MT., Schaap IA., Görlich D., Simons M. (2013). Myelin membrane assembly is driven by a phase transition of myelin basic proteins into a cohesive protein meshwork, PLOS Biol, 11(6) 



\section{Abstract}

Myelin is characterized by stacking of multiple layers of membrane compacted by MBP. The breakdown of myelin is a pathological hallmark of several autoimmune diseases of the nervous system. To assess the myelin fragmentation patterns in early stages of demyelinating disease, I employed antibody- and toxin-mediated animal models of Multiple Sclerosis and Neuromyelitis optica (NMO). Using electron microscopy of high pressure frozen samples, I could show that the temporary sequence of myelin degeneration in all the models employed starts with the vesiculation of the innermost myelin lamellae. The myelin fragmentation leads to progressive vesiculation of previously compacted myelin until the whole myelin sheath is degenerated. Furthermore, in outside-in models of demyelination, such as experimental autoimmune encephalomyelitis (EAE) and anti-MOG antibody injections, we found additional patterns of fragmentation to a lower percentage that include splitt and vesiculated myelin, as well as bulb formation.

The ultrastructural findings on the myelin breakdown are supported by the initial loss of the adaxonal protein myelin associated glycoprotein (MAG) in immunohistochemistry from the demyelinating areas prior to a loss of myelin basic protein (MBP).

To elucidate the underlying mechanism of myelin vesiculation, I focused on MBP, a protein essential for myelin assembly. An increase in intracellular calcium levels leads to the disassembly of the MBP network. The pathological phase transition by MBP molecules from a cohesive network to a soluble, non-adhesive state triggers the myelin breakdown. The QD9 antibody detects the pool of MBP molecules that are detached from the membrane and is not able to self-interact any longer. I propose that the aberrant phase transition of MBP leads to a destabilization of the myelin membrane in NMO, and possibly other demyelinating diseases.

Therefore, our data sheds light on the mechanisms of myelin disassembly and might prove useful in understanding how myelin is affected in several, yet incurable diseases, like NMO and Multiple Sclerosis. 



\title{
Chapter 1
}

\section{Introduction}

\author{
"Science is an imaginative \\ adventure of the mind-seeking \\ truth in a world of mystery."
}

Sir Cyril Herman Hinshelwood

The mammalian central nervous system (CNS) orchestrates vital biological functions, such as processing stimuli from the out- and inside of the body, coordinating motor function or regulating body functions, e.g. blood pressure or release of hormones. This vital system is composed of the brain and the spinal cord and is made up of different types of cells: the neurons and glia cells.

Neurons generate and transmit informations in the form of changes of electric potential in order to carry out the various functions of the CNS. The supportive glia cells can be further divided into microglia, astrocytes and myelin-forming cells. Microglia are the resident inflammatory cells of the brain in the defence against pathogens, as well as in the turnover of dying cells and synapses [1]. Astrocytes encircle endothelial cells that make up the blood brain barrier (BBB) and carry out a variety of tasks, such as providing trophic support for other cells, being a recycling partner of synapses [1] and maintaining ionic and water balance [2].

In vertebrates, the conduction of electrical impulses is accelerated through the use of the myelin membrane. Myelin is a spezialized membrane with a high content of lipids (70-80\%) produced by myelinating cells [3]. This membrane confers insulating properties due to a decrease in transfiber capacitance and an increase in membrane resistance. Oligodendrocytes (ODC) in the CNS and the Schwann cells in the peripheral nervous system (PNS) are the cells in charge of producing and maintaining the myelin sheath [4]. By wrapping the axon with this insulating myelin membrane, the propagation of the action potential is increased 50 to 100-fold (saltatory nerve conduc- 
tion). Myelinated neurons consume about 1000-fold less energy and space compared to unmyelinated axons conducting at the same speed [5] [6]. Furthermore, it was shown in the recent years that ODC's provide trophic support for neurons [5].

\subsection{Process of myelination}

Oligodendrocytes (ODC's) are terminally differentiated cells that derive from migratory and mitotic precursors. The precursor cells (OPC's) progress into oligodendrocyte progenitor cells that progressively mature into postmitotic myelinating oligodendrocytes.

OPC's originate from the subventricular zone in the brain and the ventral region in the spinal cord during development [7]. They undergo differentiation through several stages. Each stage is characterized by expression of different developmental markers.

OPC's display the gangliosides GD3 and A2B5 and express the intermediate filament vimentin [7] [8], transmembrane proteoglycan nerve-glia antigen 2 (NG2) or the platelet-derived growth factor receptor- $\alpha$. They proliferate and migrate in the brain and upon contact with the axon, they turn into a pre-myelinating state displaying the lipids galactosylceramide (Galc, also $\mathrm{O} 1)$ or sulfatide (O4) and expressing the proteolipid protein (PLP) and its shorter isoform DM20 [9]. Upon ensheathment of the axon, the myelinating cells first form several layers of loosely wrapped membrane around the axon and then compact the membranes upon the expression of myelin basic protein (MBP) by extrusion of the cytoplasm [10]. These mature ODC's also express 2',3'-cyclic nucleotide 3'-phosphodiesterase (CNP) and myelinassociated glycoprotein (MAG) as markers [11].

It was shown recently that OPC's can continue to divide till adulthood in mice, but resulting adult-born ODC's generate more and shorter myelinated segments, so called internodes [12] [13].

In mice, myelination starts after birth and peaks at post-natal day 20 (p20) and ends around p60. In humans, the first fibers are myelinated in the spinal cord in the second half of the fetal life and the peak of myelination occurs during the first postnatal year. Myelination is still ongoing till the second decade of life [14] [15]. It was shown that in general first thicker caliber axons become myelinated and later, also fibers with smaller diameter above $200 \mathrm{~nm}$ can acquire a myelin sheath [16] [17]. Between 200 to $800 \mathrm{~nm}$ of axonal diameter both myelinated and unmyelinated axons can be observed [18] [19]. 


\section{$1.2 \quad$ Myelin structure}

In electron microscopy (EM), the myelin sheath displays alternating electron dense and electron light layers. The dense line is also called major dense line and is formed by MBP that compacts the inner leaflets of myelin from the cytoplasmic side. On the other hand, PLP adheres the outer membrane surfaces together and these opposing membranes then form the electron light or intraperiod line [20]. The periodicity of the lamellae was determined to be $12 \mathrm{~nm}$ [14] and an optimal degree of myelination is marked by the g-ratio of 0.6 to 0.7 determined by the inner axonal diameter divided by the outer diameter of the myelinated axon [21] [22]. Changes in the g-ratio can result in a decreased conduction velocity [23].

Axons in the PNS are myelinated by one Schwann cell, whereas in the CNS, mutliple axons can be myelinated by a single ODC. In fact, an ODC can form up to 80 internodes on small caliber axons. On thicker axons, fewer, but longer internodes are formed with thicker myelin sheaths [16] [24] [25]. Hence, there is a correlation between the axonal diameter, the number of lamellae and length of the internode and this vast amount of myelin produced by ODC's renders them the most powerful membrane producer in the body [26]. The myelin sheath is an extremely stable structure with a slow turn-over rate of membrane as myelin proteins are among the longest-lived proteins in mice as shown by an in vivo pulse chase labeling [27].

Myelin is polarized in two domains: compact and non-compact myelin. Noncompact myelin contains the cytoplasm and the vesicular machinery of the cell, as well as the proteins CNP and MAG among others. In contrast, the compact myelin is devoid of cytoplasm due to the compaction of the cytoplasmic leaflets by the peripheral MBP protein. The protein segregation results at least in vitro from the extrusion of proteins with big cytosolic domains from the MBP-positive areas [28].

The internodes are separated from non-myelinated segments that constitute the nodes of Ranvier (see Figure 1.1). At the node of Ranvier, the axonal membrane is in direct contact with the extracellular space and contains a high density of voltage-gated sodium channels and other channels, as well as transmembrane and cytoskeletal proteins like Neurofascin-186, ankyrin $\mathrm{G}$ and spectrin [29]. Here, the axon potential is propagated from one node to the next sparing the internode (saltatory signal conduction).

At the edge of the internode is the paranode that contains adhesion proteins to maintain the contact to the axons. The paranode is filled with cytoplasm and winds around the axon. By forming tight junctions of contactinassociated protein (Caspr) and contactin on axonal side and Neurofascin155 on the glia membrane [30], the paranode maintains a strong axon-glia 


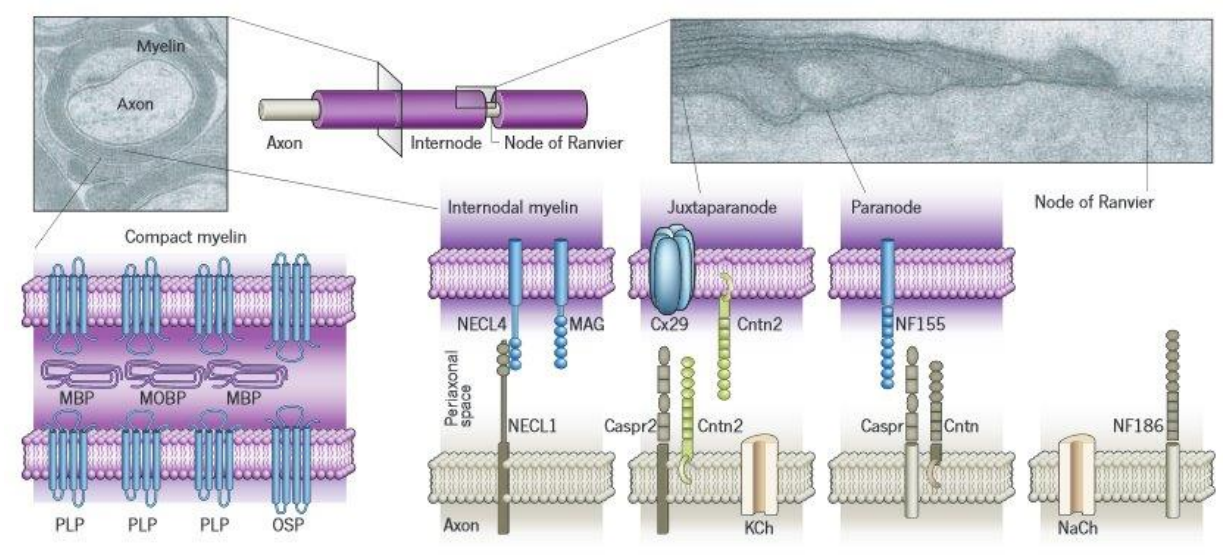

Figure 1.1: Myelin structure and protein distribution at the axon-glia junction in the CNS The upper panel shows the ultrastructure of a cross-section of a myelinated axon, schematically depicted in the middle with the internode and node of ranvier, and the paranode on the right side. The lower panel depictes the distribution of proteins at the axon-glia interface along the different myelin regions: compact myelin, periaxonal region, juxtaparanode and paranode. Abbreviations: Caspr, contactin-associated protein; Cntn, contactin (Cntn2 / Tag1); Cx29, connexin $29 \mathrm{kDa}$; KCh, fast potassium channels; MAG, myelinassociated glycoprotein; MBP, myelin basic protein; MOBP, myelin oligodendrocyte basic protein; NaCh, voltage-gated sodium channels; NECL, nectin-like protein/synCAM; NF155/186, neurofascin $155 \mathrm{kDa} / 186 \mathrm{kDa}$; OSP, oligodendrocyte specic protein or Claudin 11; PLP, proteolipid protein. The figure is adapted from [5]. Reprint by permission from Macmillan Publishers Ltd: Nature, copyright (2010).

adhesion. The importance of the adhesion molecules are highlighted by disorganized paranodes and subsequent myelin degeneration upon loss of neurofascin. This causes ataxia and pre-mature death in these knock-out mice [31].

The juxtaparanode is adjacent to the innermost paranodal junction and further maintains the axoglia adhesion by expression of immunoglobulinlike family of adhesion molecules, such as Tag-1 [32]. Glial Tag-1 was shown to interact homophilically with axonal Tag-1 that forms a complex with Caspr2 on the axonal side [33]. Furthermore, this macromolecular complex clusters potassium channels, which is crucial for repolarisation of the axonal membrane.

Another domain in myelin is the periaxonal region: The non-compacted innermost layer of myelin harbours most of the metabolic activity, as well as the membrane growth [34]. The proteins MAG and Necl-4 reside in this compartment [35]. The outermost layer, the abaxonal domain, contains MOG [36]. 


\subsection{Myelin function}

Myelin is crucial to ensure optimal signal propagation along myelinated axons by saltatory signal conduction, but it also provides trophic support to axons. The metabolic support of axons by ODC's was shown as pyruvate or lactate can be transferred from ODC's to neurons via monocarboxylate transporters [37]. This important function of ODC's is highlighted by the axonal damage and neuronal loss upon inhibition of the glial lactate transporter [5] [38].

These functions become evident as myelin loss causes reduced signal conduction velocity and eventually cause axonal degeneration and severe disabilities [5]. Studies on the knock-out mice for MAG, PLP or CNP show that the loss of these proteins first leads to a normal myelin ultrastructure, but myelin function seems to be impaired resulting in late-onset and progressive neurodegeneration [39] [40] [41] [42]. Additionally, in demyelinating lesions of Multiple Sclerosis (MS) patients, axonal swellings and impaired transport was reported.

Despite that, upon complete absence of compacted myelin in the naturally occuring MBP mutant shiverer, no axonal damage or degeneration could be observed, which suggests that the unmyelinated axon can survive on its own, although a myelinated axons needs an intact sheath for its maintenance.

\subsection{Molecular composition of myelin}

Myelin is highly enriched in lipids with $80 \%$ lipids and only about $20 \%$ of proteins (of dry weight), when compared to a normal plasma membranes with an equal ratio of proteins and lipids [26] [43]. This high lipid content of myelin is an essential feature for its insulating functions and due to its low density, allows an biochemical purification via density gradient centrifugation [44].

\subsubsection{Lipid composition of myelin}

Lipidomics of biochemically purified myelin showed that the most abundant lipids are glycosphingolipids - especially galactosylcerebrosides (Galc), sulfogalactosylceramides (sulfatides) and cholesterol (see Figure 1.2). Myelin has a very specific lipid composition as it contains only low amounts of polyunsaturated, but instead more very long fatty acids resulting in a tighter packing of lipids and stronger hydrophobic interactions [45]. In the following paragraphs, the different classes of lipids are presented with their biological role. 


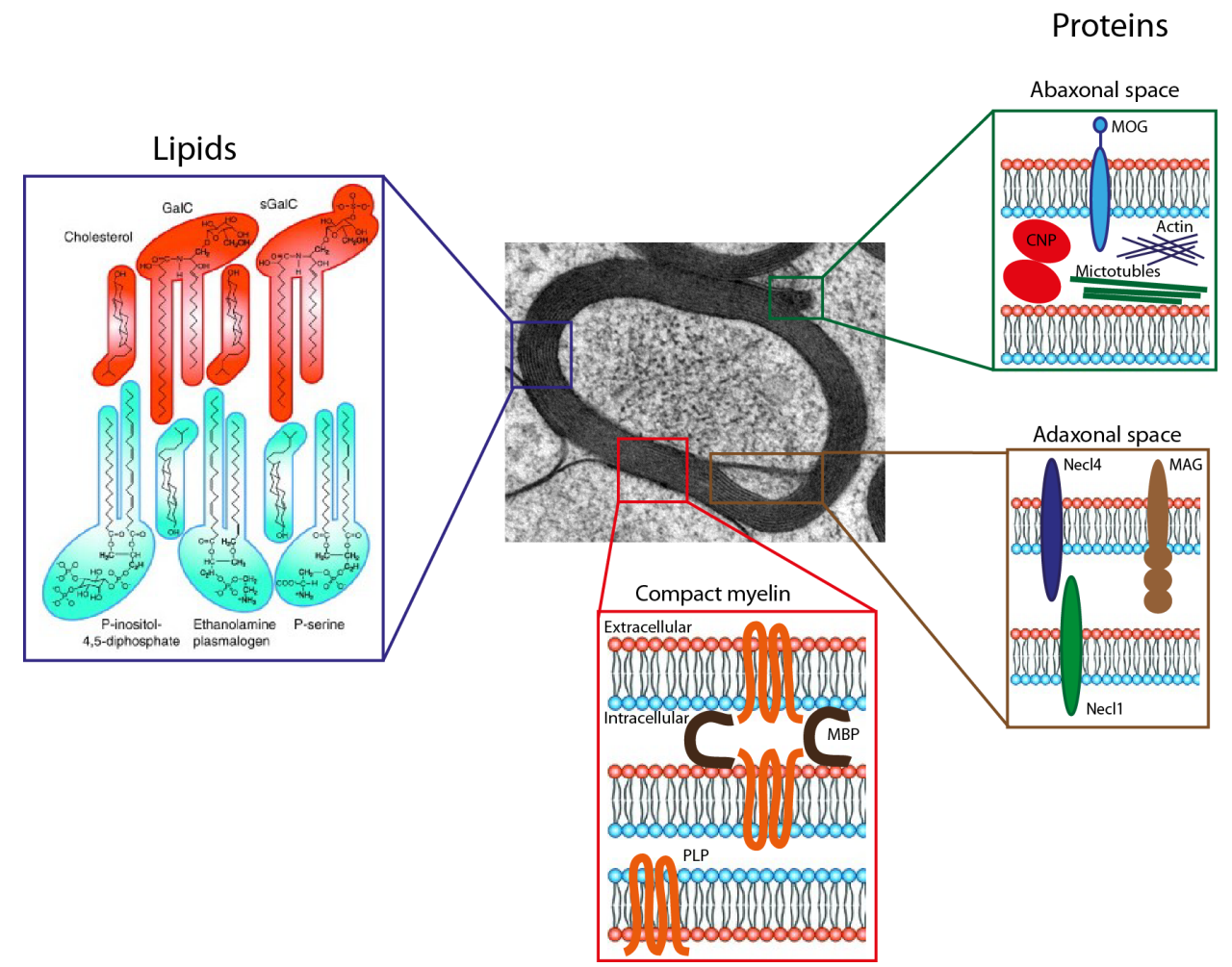

Figure 1.2: Overview of the most abundant proteins and lipids and their subcellular localisation in myelin

On the left side, the most abundant lipids of myelin are depicted, whereas the right side shows the most common proteins of the myelin sheath in the different subcellular regions. Abbreviations: Galc, Galctosylceramide; sGalc, sulfogalactosylceramides; MOG, myelin oligodendrocyte glycoprotein; CNP, 2',3'-cyclic nucleotide 3'-phosphodiesterase; MBP, myelin basic protein; PLP, proteolipid protein; NECL, nectin-like protein/synCAM; MAG, myelin-associated glycoprotein. 


\subsubsection{Glycerolipids}

Glycerolipids are the main constituents of membranes and derivates of phospathic acid that are either linked to an aliphatic alcohol or an unsaturated alcohol (plasmalogens). The fatty acid ester (at sn1 and sn2) forms diacylglycerol that is the basic structure for phosphodiester linkage to other bases for the generation of this diverse lipid class.

Phosphatidylcholine (PC) is a zwitterionic phospholipid with choline as head group that is the main component of membranes and frequently found in the outer leaflet. Phophatidylethanolamine (PE) is a zwitterionic lipid with glycerol and two fatty acids linked to phosphoric acid [20]. Due to its conical shape with the small head group, it can induce negative curvature [46]. Furthermore, PE is a marker for autophagy and the basis for the synthesis of most other phospholipid classes.

Phosphatidylserine (PS) is a anionic phospholipid that can also act as a second messenger. This lipid is exclusively located in the inner leaflet due to aminophospholipid translocases. Upon apoptosis, PS is exposed towards the extracellular matrix by calcium-sensitive scramblases and impaired activity of aminophospholipid translocase. If PS is exposed on the outer leaflet, it is acting as an "eat me"-signal for macrophages and phagocytes [47] [46]. Additionally, PS is an important interaction partner for cationic proteins, such as MBP, GTPases and K-Ras [48] [49].

Plasmalogens are glycerophospholipids with an fatty acid moiety at sn1, which segregate lipids into lateral domains [50]. This lipid class exhibits an increased synthesis upon development and myelination [51]. Mice lacking dihydroxylacetone phosphate acyltransferase, a key enzyme in the plasmalogen synthesis display a decreased myelin synthesis in the optic nerve and cerebellum [52].

\subsubsection{Phospatidylinositols}

Phospatidylinositols are anionic lipids enriched in the inner leaflet that regulate a wide range of metabolic processes in cells and undergo reversible and transient changes in their head group [46]. The two most important lipids of this class, namely phosphatidylinositol-4,5-bisphosphate (PIP2) and phosphatidylinositol- 3,4,5-trisphosphate (PIP3) are usually kept in a balance inside the ODC's and can be interconverted via phosphorylation or dephosphorylation.

PIP2 is produced by dephosphorylation of PIP3 by phosphatase and tensin homologue (PTEN). This phospholipid has a negative charge of -4 at physiological $\mathrm{pH}$ [53] and binds positively charged proteins, such as Myristoylated alanine-rich C-kinase substrate (MARCKS) proteins like MBP and K-Ras. Thus, PIP2 recruits proteins to the membrane and hence, regulate enzyme 
activity, ion channels [54] and binding of scaffolding proteins [53]. Moreover, membrane trafficking [55] and cytoskeleton dynamics [56] [57] are controlled by this lipid.

PIP3 is a major signaling molecule in the plasma membrane that can lead to cell growth and survival by activation of the Akt pathway, as well as the polymerization of actin. In ODC's, activation of the Akt pathway results in enhanced myelination [58]. It can be generated by the phosphorylation of PIP2 via the PI3 kinase [46].

\subsubsection{Sphingolipids}

Sphingolipids are versatile lipids consisting of ceramide, sphingoid base and an amide-linked fatty acid that reside in the outer leaflets. Their hydrophobic ceramide moiety is embedded in the membrane, whereas the sugar heads face towards the extracellular matrix [59].

Glycosphingolipids have a sugar head group linked to ceramide (cerebrosides) and are precursors for globosides that are neutral lipids containing more than two sugar residues; or precursors for gangliosides linked to sialic acid (acylneuraminic acid). This lipids class -particularly galactosylceramide (galactocerebroside) and its derivative sulfatide -are highly enriched in the brain, especially in the outer myelin leaflets [60]. These lipids self-aggregate to form an ordered phase. They are usually linked to very long chain fatty acids (22 to 24 carbons) that are added by the ceramide synthase 2 to the sphingosine. Ceramide synthase 2-deficient animals exhibit an progressive loss of MBP and myelin [61]. It was further shown that the myelin formation of these animals was not impaired, but the myelin maintenance severely affected [62]. Transgenic mice lacking key enzymes in the metabolic pathway for these lipids display abnormal myelin with disrupted paranodes [63] [64] [65].

In humans, a disrupted degradation of Galc and/or sulfatides can cause globoid leukodystrophy (Krabbe's disease) or metachromatic leukodystrophy [25]. The former is caused by a deficiency in the enzyme galactocerebroside$\beta$-galactosidase that carries out the degradation of Galc and the toxic side product galactosylsphingosine (psychosine). The resulting accumulation is toxic for neurons, ODC's and microglia. Together with the apoptosis of ODC's and disrupted myelination, this pathology can be held responsible for the fatal disease in infants [66]. The fatal metachromatic leukodystrophy is caused by a accumulation of sulfatides due to the deficiency in arylsulfatase A and subsequent progressive demyelination and neurological symptoms [67].

Sphingomyelin contains a phosphocholine head group and is together with cholesterol the main constituent in lipid rafts [68]. Hence, this lipids medi- 
ates a decreased fluidity and an increased membrane stability [69]. Among the biological function shown to be mediated by this lipid are signal transduction, lipid and protein trafficking [70].

\subsubsection{Cholesterol}

Cholesterol is an essential membrane component that is necessary for membrane permeability, as well as fluidity [71] and regulates the activity of transmembrane proteins [72] and ion permeability [73]. This relatively small, amphiphilic lipid interacts with the polar heads of other lipids via its hydroxylgroup, whereas the hydrophobic ring structure interacts with hydrophobic lipid side chains and leads to increased rigidity of the membrane. The presence of cholesterol forces hydrocarbon chains in an extended conformation, hence leads to a thickened membrane. Furthermore, this steroid lipid segregates other lipids laterally through hydrophobic mismatch in a thicker ordered and thinner disordered phase [74].

Cholesterol is produced in large amounts during myelination and the synthesis is decreased after completion of myelination that possibly due to a slow turnover and long half-life of 4 to 6 months [75]. As cholesterol cannot be cross BBB, it needs to be synthesized locally [73] [75] or horizontally transferred from surrounding cells [76]. ODC's are capable of de novo synthesis or uptake [76]. Disturbances in cholesterol levels are associated with neurodegenerative diseases, such as familial Alzheimer's disease [77], SmithLemli-Optiz syndrome and desmosterolosis [78]. Neurological symptoms like ataxia and tremor, as well as myelin deficiency were also observed for knockout animals of squalene synthase that catalyzes the first committed step to the sterol biosynthesis [76]. This highlights the importance of cholesterol as an essential component of healthy myelin.

Every single lipid class has a biological function in the healthy brain and upon disruption of the homeostasis, frequently myelin pathology is acquired highlighting the importance of maintaining a certain lipid composition in myelin.

\subsubsection{Protein composition of myelin}

The myelin membrane consists of $20-30 \%$ of proteins and the most abundant proteins are proteolipid protein (PLP) and myelin basic protein (MBP) representing $17 \%$ and $8 \%$ of total myelin proteins respectively. Other abundant proteins in the CNS are MAG, MOG, CNP, MAL, claudin-11, neurofascin 155 and opalin/Tmem 10 [3] (see Figure 1.2). Many of proteins of the myelin sheath have four transmembrane domains and the alterations of these tetraspanins themselves or their expression levels cause neurological diseases 
such as Charcot-Marie-Tooth disease, which is caused by changes in PLP. This highlight once more that the correct myelin assembly and maintenance is crucial for axonal function.

In the following paragraphs, I introduce the proteins studied in this thesis.

\subsubsection{Proteolipid protein}

Proteolipid protein (PLP) is the most abundant protein and the main function of this tetraspanin is the stabilization of the interperiodal line via its extracellular domains [79]. The PLP gene is localized on the X chromosome and is alternatively spliced to generate two isoforms. The shorter isoform named DM20 lacks 35 amino acids on its intracellular domain [80]. This $30 \mathrm{kDa}$ protein contains four $\alpha$-helices, as well as a highly basic region and interacts with various lipids [81], especially cholesterol and Galc [82].

Mutations causing misfolding of PLP lead to severe myelin deficiency in the CNS of the "Jimpy" mice [83]. Knock-out animals for PLP have a normally compacted myelin sheath with condensed intraperiodal line and display axonal swellings and degeneration [40] [84]. In humans, gene duplication or deletion in PLP are associated with Pelizaeus-Merzbacher disease, CharcotMarie-Tooth disease and X-linked spastic paraplegia [85]. In vitro, the overexpression of PLP results in an accumulation together with cholesterol in the endosomes [86]. In vivo, feeding of cholesterol-rich chow to the PLP transgenic mice reduces the intracellular accumulations of PLP and cholesterol and thus, suggest that cholesterol is promoting the PLP incorporation in the membrane [87].

\subsubsection{Myelin basic protein}

Myelin basic protein (MBP) is the second most abundant protein of myelin and necessary for the compaction of the inner leaflets of the myelin membrane[88]. At physiological $\mathrm{pH}, \mathrm{MBP}$ has a +19 charge and electrostatically interacts with negatively charged lipids, mainly PS and PIP2 [89]. This interaction triggers the self-assembly of MBP and therefore, allows the formation of an MBP network. This network allows MBP to extrude the cytoplasm and act like a molecular sieve and hence, restricts the entry of proteins with a spacious cytosolic domain in the compacted areas [28]. MBP interacts with SH3-domain containing proteins (e.g. Fyn kinase), but also with actin and tubulin [90] [91] [92]. MBP was also shown to be the major calcium-dependent calmodulin binding protein [93] via its C-terminus [94] [95]. 


\subsection{Transcriptional and translational regulation of MBP ex- pression}

The MBP gene complex called GOLLI (genes of the oligodendrocyte lineage) encodes two families of proteins resulting from differential splicing of a single mRNA with three different start sites, namely the classical MBP and the golli proteins [96] [97] [98] [99].

The golli proteins consits of BG21, J37 and TP8 [100]. These proteins regulate the calcium influx in ODC's [101]. They are expressed in myelin-forming cells, neurons of CNS and PNS [102], macrophage cell line, B cells and in tissues of the immune system like the thymus and spleen [103].

The classical MBP proteins in human CNS are the MBP isoforms of 21.5, $20.2,18.5,17.24,17.22 \mathrm{kDa}$ with $18 \mathrm{kDa}$ being the most abundant one [99] [98] [104]. In mice and rats, the following isoforms are expressed: 21.5, 20.2, $18.5,17.24,17.22,14 \mathrm{kDa}$ with the $14 \mathrm{kDa}$ isoform as most abundantly expressed one [105]. The most common isoforms, 14 and $18 \mathrm{kDa}$ containing the exons 1, 3, 4, 6 and 7 are expressed later in myelination and associate to the membrane [106]. The other exon 2-containing isoforms are expressed early during development [97] and are located in the nucleus [106]. Their function is not yet understood.

Due to the highly basic properties of MBP, the translated protein will immediately bind to the membrane and start compacting the leaflets. Hence, the transport and local translation of the mRNA in the myelin compartment is crucial for an appropriate spatiotemporal pattern of compaction. The MBP mRNA was shown to be transported in RNA granules and enriched in crude myelin fractions [107] and at the sites of myelin membranes [108] allowing local translation [109] [110]. The 3'-untranslated region contains a 21 nucleotides long domain that is sufficient for RNA trafficking to the myelin compartment [111]. The translation of the mRNA is repressed due to the interaction with hetereogeneous nuclear ribonucleoprotein A2. The translational repression is released upon phosphorylation of the ribonucleprotein by Fyn kinase [112]. Conclusively, signalling via Fyn kinase regulates expression of the MBP protein.

\subsection{Post-translational modifications of MBP}

MBP exhibits a high degree of post-translational modifications, such as phosphorylation, citrullination (deimination), deamidation, N-terminal acylation, oxidation of methionine and arginine methylation. These modifications decrease the charge of the protein and hence, have an impact on the structure of MBP, but also its adhesive capacity. Moreover, acetylated [113] and citrullinated MBP [114] was shown to elicit an immune-response (see Figure 1.3).

Most studies focus on citrullination and phosphorylation of MBP. Phosphorylation of serine 7 by protein kinase $\mathrm{C}$ leads to an increase in secondary 
structure [115] resulting in an increased interaction with tubulin [116] and decreased association with actin [116] [117]. Phosphorylation of serine and threonine residues of MBP was shown to regulate the attachment to the membrane and is implicated during development [118] [115], ageing and MS [116] [119]. During demyelination in MS or in the spontaneously demyelinating mouse mutant ND4, the phosphorylation of MBP is largely decreased. The protein is then targeted to a different microdomain in the myelin membrane suggesting that phosphorylation affects its adhesive properties [119] [120] [121] [122]. Due to the decrease of phosphorylation, the three dimensional structure of MBP becomes less compact due to the lack of $\beta$-sheet stabilization and hence, more vulnerable for proteases [123]. Kim et al. found that the lack of phosphorylation in MS samples and those of the ND4 mutant, can be correlated with an increase in mono-or dimethylation of arginine 107 in these samples. This suggests that some post-translational modifications confer resistance to degradation by proteases resulting in a longer half-life [119].

Citrullination or deimination of arginine residues by peptidyl arginine deiminase (PAD) results in the irrevesible generation of citrulline and results in the decrease of the overall change by 1 per citrulline residue. This was shown to result in significant changes of the adhesive properties of MBP and moreover, its ability to organize the myelin membrane [124] [125] [126]. The least modified version of MBP that contains the highest charge is termed $\mathrm{C} 1$, whereas the $\mathrm{C} 8$ variant exhibits 6 to 8 deiminated residues. The latter variant was shown to have a more open structure [127] with a shorter amphipathic helix (V83-T92) that was more exposed to the surface. Hence, it is vulnerable to digestion with cathepsin $\mathrm{D}$ [128] that cleaves between the two phenylalanines contained within the amphipathic helix. Cathepsin D is localised in myelin around active plaques [129] and its activity is increased in MS patients [130]. Citrunillation of MBP is increased during active disease and correlates with MS severity [131] [132] and the increase in PAD 2 expression [133] [134]. Hence, citrullination of MBP by PAD 2 and the subsequent degradation of MBP by cathepsin D seems to play a role in MS. In fact, it was shown that $90 \%$ of MBP is deiminated in the Marburg's syndrome, whereas $45 \%$ of MBP are citrullinated in chronic MS compared to $20 \%$ in normal brain [135].

Electrostatic interaction of positively charged arginine and lysine residues with negatively charged lipids, such as PS or PIP2 are crucial for the compaction of myelin. Post-translational modifications decrease the charge of MBP and hence disrupt the adhesion to the membrane. For the charge isomers of MBP, the electrostatic interactions with the membrane decrease, while the hydrophobic interactions increase leading to a pertubation of the membrane stacks. This results in a changed conformation of MBP allowing proteolytic degradation and fragmentation of membrane stacks [125]. 


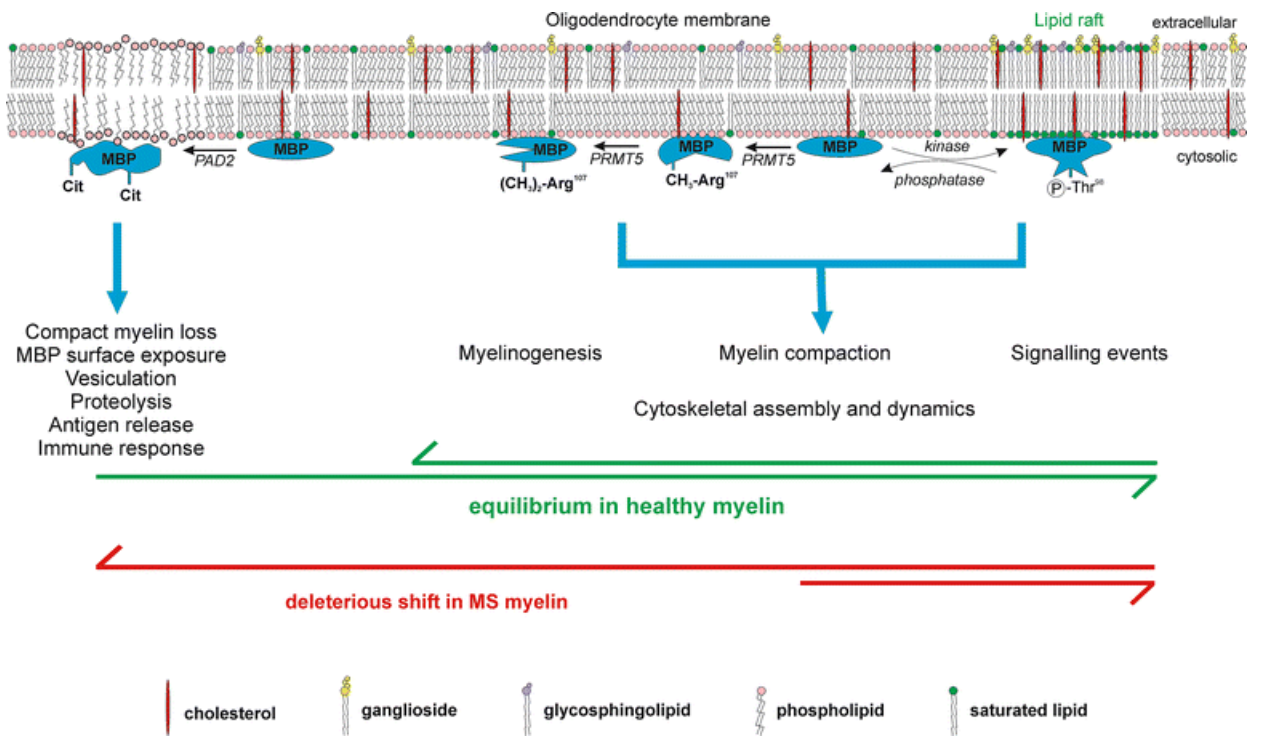

Figure 1.3: Simplified overview of the post-translational modifications and their implication on the localisation and function of MBP

In healthy myelin, there is a equilibrium of different post-translationally modified MBP variants. Methylation, such as mono- or dimethylation on arginine (Arg) 104 (murine sequence numbering of $18.5 \mathrm{kDa}$ ), as well as phosphorylation at various serine or threonine residues by several kinases are relevant for myelinogenesis, myelin compaction and resistance to proteolysis. Phosphorylated Thr95 by MAP-kinases leads to partitioning of $\mathrm{MBP}$ in lipid rafts and is important for signaling. Deimination at specific Arg to citrulline (cit) residues by peptidylarginine deiminase 2 (PAD2) is implicated in demyelination as it renders MBP vulnerable to proteolysis. Upon demyelination, the equilibrium of the different post-translationally modified versions of MBP is disrupted initiating a cascade of events leading to demyelination. Figure taken from [131]. 


\subsection{Interaction of MBP with lipids}

As already mentioned above, MBP binds negatively charged lipids via electrostatic interactions with the membrane. If MBP is added to liposomes with neutral and negatively charged lipids, it preferentially binds negatively charged lipids [89] resulting in aggregation of the liposomes and multilayer formation [136]. Furthermore, the $\mathrm{N}$ and $\mathrm{C}$ terminal part of the protein can independently associate with lipids as positively charged residues are distributed over the whole sequence [137]. MBP adsorption to a lipid monolayer leads to rearrangements and morphological changes [138], such as clustering of PIP2 on large unilamellar vesicles [49].

Post-translational modifications of MBP, such as increased citrulline content as detected in MS patients can decrease the affinity towards the membrane due to their decrease of the overall charge of the protein [124] [119]. Moreover, changes in the local $\mathrm{pH}$ or ionic strength can alter the adhesive properties of MBP in vivo [139] and in vitro. Interestingly, increased ionic strength causes MBP detachment from PC/PS containing vesicles, but not from liposomes of inner leaflet composition [140] indicating that hydrophobic interactions are also involved. This is further supported by the finding that MBP affects the movement of fatty acids buried deep within the membrane [141].

MBP was shown to increase the lipid order to a gel phase by clustering myelin lipids and modulating their packing [142] [143] [144]. Lateral organization of membrane domains was observed for PIP2, but shown to be due to nonspecific electrostatic interactions and partially dependent on cholesterol [49]. MBP is also a sensor for lipids present in the outer leaflet, such as Galc and sulfatides. Clustering of Galc or sulfatides with antibodies against these epitopes results in a redistribution of MBP and depolymerization of the cytoskeleton in ODC's [145] [146]. The bidirectional influence of MBP and Galc was further proven as the inhibition of Galc leads to a redistribution of MBP from rafts into non-raft fractions. Furthermore, if MBP levels are decreased using an MBP siRNA, the formation of Galc clusters is inhibited [147]. This provides strong prove that MBP and Galc localise to the same membrane domains (although Galc resides in the outer leaflet) that are CHAPS-insoluble microdomains.

\subsection{Structure of MBP}

MBP belongs to the family of intrinsically unstructured proteins (IUP) that usually contain a high degree of random coils [148], as well as a low hydrophobicity and a high overall charge due to their high content in glutamic acid, arginine, serine and lysine residues [148] [149]. Their high positive charge and leads to the formation of basic amino acid-rich effector domains that enable the binding of actin and calmodulin etc. These natively unfol- 
ded proteins are difficult to crystallize alone as their structure is stabilized upon interaction with other proteins. IUP's have an extended structure due to the high intramolecular repulsion of the charged residues [150], hence a large surface to interact with other proteins or lipids. Unlike other family members of the IUP's like myristoilated alanine-rich $\mathrm{C}$ kinase substrate and $\mathrm{K}$-Ras, MBP has its basic residues distributed evenly over the whole protein [53] and its elongated structure allows it to interact with the charged lipids of the membrane to compact the leaflets.

MBP has a disordered structure in solution and only upon binding to charged lipids, it acquires $\alpha$-helices and $\beta$-sheets [151] [152]. Computational three dimensional modeling of EM observations of MBP in presence of lipids generated an extended $\mathrm{C}$ shape (see Figure 1.4) with antiparallel $\beta$-sheets forming the central core and loop regions on either side [153] [127]. Adsorption experiments of MBP to a silicium dioxide surface suggest that MBP adsorbs to a lipid bilayer in a swollen state before slowly changing into a more compact structure of $3 \mathrm{~nm}$ thickness. The formation of adhesive bridges with nearby membranes seems to be the key for the strong adhesion of MBP [154]. Vassall and colleagues propose a three state mode (disordered/intermediate/ $\alpha$ helical MBP) for the structure of MBP: The disordered state of MBP in solution has low compaction with little regular secondary structure, whereas the intermediate state of MBP is still disordered, but contains a globally more compact structure. The $\alpha$ helical state of MBP is less compact, but more extended than the intermediate form. This model suggests a reversible, global change in conformation with energetically distinct conformations that have an effect on the multifunctionality of MBP [155]. These results suggest that the changes in structure of MBP have implications for its role in membrane compaction in vivo.

In membrane environment, three domains in the murine $18.5 \mathrm{kDa}$ isoform of MBP form an $\alpha$-helix as determined by electron paramagnetic resonance spectroscopy and site-directed spin labeling [94]: T33-D46; V83-T92 and T142-L154. The $\alpha$-helix made by these domains is considered to be an amphipathic helix that has a hydrophobic face penetrating the membrane and a hydrophilic face facing the cytoplasm. They can sense the physical properties of the membrane and can induce membrane curvature. Generally, these helices have a weak and reversible binding to the membrane that is dependent on hydrophobic interactions. Amphipathic helices require anionic lipids to bind the membrane and subsequently, undergo a conformational change, but hydrophobic interactions are important for the binding strength of the helix [156].

Interestingly, one of the amphipathic helices (V83-T92, murine sequence numbering) was shown to be an immunodominant epitope as it is the minimal epitope for $\mathrm{T}$ cell recognition of $\mathrm{MBP}$ with the highest affinity for the MHC class II haplotype [157] [158]. Therefore, this domain is the most 

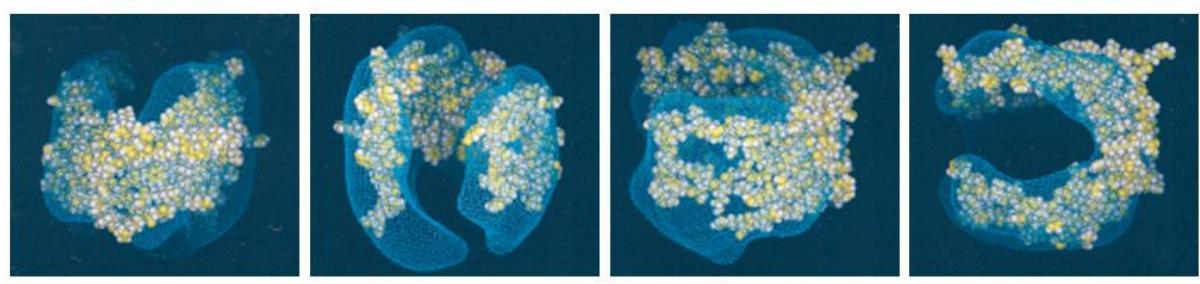

Figure 1.4: Electron microscopy-based reconstruction of $18.5 \mathrm{kDa}$ MBP from bovine brain reconstituted with gangliosides

The protein is rotated in a $90^{\circ}$ angle from left to right around the vertical axis. Figure taken from [164].

investigated and was shown to be highly conserved. The immunodominant domain only forms an amphipathic helix in the membrane-via its phosphoinositide binding site [159] [160]. Hence, this domains is buried within the membrane at a $9^{\circ}$ tilt with lysine 88 penetrating the deepest. This places the charged residues in the polar membrane region facing the cytosol. Furthermore, lysine 88 positions the hydrophobic part of the helix deeper in the membrane and leading to a stronger binding. The deep penetration thus results in strong membrane binding without perturbating the stability of the bilayer [161] [160]. The phenylalanines within the amphipathic helix interact hydrophobically with the membrane as their mutation lead to an increased mobility and an decreased membrane penetration [160] [162].

The amphiphatic helix also contains residues that can be modified posttranslationally, such as the phosphorylation of the threonine residue or the citrullination of the arginine residues that results in destabilisation of the helix and C-terminal displacement of the membrane [163]. Citrunillation of the immunodominant epitope results in an more exposed helix of shorter length and was shown to be more vulnerable to cleavage with cathepsin D implicated in MS [49].

Additional domains exist in the C-terminal part of the protein as it harbours a calmodulin binding site and a proline-rich region that is a classical Src homolog 3 binding site for Fyn kinase [92].

\subsection{Shiverer mice}

The importance of MBP for myelin formation is highlighted by the naturally occurring mouse mutant shiverer. These mice have an autosomal recessive deletion in exon 2 to 7 leading to a loss of MBP expression. Two weeks after birth at the peak of myelination, these mice start to shiver and later develop tonic convulsion, tremor and seizures before dying prematurely between 8 to 11 weeks [165]. While the myelin in the PNS is normal, CNS myelin lacks the major dense line [166] and shiverer mice have severe hypomyelination 
[167]. Fragmentation profiles of myelin can already be found at 2 to 3 weeks of age [168] indicating that the initial wrapping of the membrane around the axon is not affected in these mice, but the myelin cannot be maintained. In vitro, shiverer ODC's show abnormal process formation and have a perturbed cytoskeleton [169].

The shiverer phenotype can be rescued upon re-expression of the full length MBP gene encoding for all isoforms [170] or only the $14 \mathrm{kDa}$ MBP isoform [171]. In line with that, expression of an antisense cDNA induces a shivering response [172]. Another naturally occurring mouse mutant named myelin deficient that has an inversion of exon 2 in the duplicated MBP gene and hence, forms an antisense RNA that leads to an decreased MBP expression and hypomyelination [173] [174]. These findings highlight that MBP is not required for the intial wrapping, but crucial for compaction and maintenance.

\subsubsection{Myelin oligodendrocyte glycoprotein}

Myelin oligodendrocyte glycoprotein (MOG) is a transmembrane type I protein from the immunoglobulin superfamily [175] and highly conserved among species. The single immunoglobulin domain is located at the $\mathrm{N}$-terminal side of the protein and exposed towards the extracellular surface, whereas the hydrophobic part in the cytosolic domain is thought to bind to the membrane [176] [175]. MOG contains a single glycosylation site that is linked to oligosaccharides to a high extent. This minor constituent of myelin $(28 \mathrm{kDa})$ is located at the outer tongue of the myelin sheath [36] and on the surface of mature ODC's in culture [177]. Its easily accessibility from the extracellular space renders MOG a highly susceptible target and given a possible explanation for its implication as an autoantigen in MS or EAE [178] [175]. The biological function is still unknown, but MOG is thought to be an adhesive molecule that interacts with the cytoskeleton [146] and regulates microtubule stability [179]. In contrast to WT mice, Mice deficient in MOG that are immunised with whole myelin, display only a mild phenotype. This highlights the anti-MOG immune response contributes to the pathology of EAE induced with myelin [180].

MOG is not expressed in the thymus and hence, MOG-reactive lymphocytes may exist in the periphery [175] [180] and these can evoke the CNS inflammation. It is also the one of the few CNS autoantigen that was shown to induce EAE and have a demyelinating antibody response. Additionally, MOG antibodies are shown to augment the clinical symptoms and demyelination in EAE [181] and especially those recognizing conformation-dependent epitopes have demyelinating potential in EAE [182]. In the serum of MS patients, MOG antibodies are rarely detected, but were shown to be present 
in small subset of neuromyelitis optica (NMO) and acute demyelinating encephalomyelitis patients [183] [178].

Intracerebral injection of anti-MOG IgG causes myelin changes and an altered expression of MBP and axonal proteins (Caspr, Ankyrin G), albeit no immune reaction was elicited and the neurons and astrocytes were preserved [184].

\subsubsection{Myelin-associated glycoprotein}

Myelin-associated glycoprotein (MAG) is a minor component of myelin that is found in the periaxonal space of the internode [185] [186]. This transmembrane protein type I $(100 \mathrm{kDa})$ has five immunoglobulin-like domains and two alternatively spliced C-termini [187]. The short MAG isoform (67 $\mathrm{kDa}$ ) is broadly expressed later in development in all non-compact myelin membranes, whereas the long MAG $(71 \mathrm{kDa})$ is expressed earlier in development and restricted to the periaxonal space [35].

MAG is negatively charged due to the sialic acid or sulphates residues attached [35] and it can bind other sialic acid containing oligosaccharides on glycoproteins or gangliosides [188]. Further interaction partners of MAG are tubulin [189], protein kinase A and C [190], Fyn kinase, phospholipase C [191], Src kinase [192]. MAG has been implicated in mediating the axon-glia adhesion during myelinogenesis and signal transduction via Fyn kinase [193] [194].

Transgenic mice deficient in MAG show a delay in myelination [195] with normal myelin ultrastructure and an abnormal periaxonal region [196]. Upon ageing, progressive axonal degeneration was also shown for these animals [41].

\subsubsection{2',3'-cyclic nucleotide 3'-phosphodiesterase}

2',3'-cyclic nucleotide 3'-phosphodiesterase (CNP) is a membrane interaction protein that is present in the non-compact myelin at the inner and outer tongue, as well as the lateral loops [197]. This protein has a $2 \mathrm{H}-$ phosphodiesterase activity that allows the hydrolysis of 2',3'-nucleotides to 2 '-nucleotides. As these nucleotides are not present in the brain, the enzmyatic function is still not understood. Two isoforms (46 kDa and $48 \mathrm{kDa}$ ) are generated from CNP transcripts by alternative splicing or translation from two different start site [198]. The proteins display a wide range of post-translational modifications including acylation, phosphorylation and isoprenylation [199]. CNP is an ODC marker that is expressed early during development and is shown to interact with the cytoskeleton. Furthermore, CNP is involved in RNA binding and trafficking [42] and regulates process 
outgrowth by microtubule assembly [200]. CNP knock-out mice have normal myelin ultrastructure, but altered paranodes and display axonal swellings and degeneration leading to a premature death [42]. Hence, CNP also seems to be involved in the maintenance of the axon-glia interaction.

\subsection{Demyelinating diseases}

Demyelinating diseases can affect the CNS or the PNS. It can be further distinguished between diseases, in which myelin in not properly produced or maintained and diseases, where myelin is damaged by external means. The former category consists of genetic disorders such as leukodystrophies (e.g. Krabbe disease, Pelizaeus-Merzbacher disease), whereas leukoencephalopathies are caused by viruses (e.g. John Cunnigham virus, papovavirus). Furthermore, metabolic dysfunctions, such as central pontine myelinolysis contribute to this category.

The demyelinating diseases due to myelin damage by external means are often acquired idiopathic disorders marked by loss of myelin and inflammation. The most frequent autoimmune diseases targeting myelin are: Multiple Sclerosis (MS) and its acute variant, the Marburg's syndrome; neuromyelitis optica (NMO), Balo's concentric sclerosis, acute disseminated encephalomyelitis and its hyperacute variant hemorrhagic leukoencephalitis. The diagnosis of these diseases is taken upon the clinical history, neurological examination, cerebrospinal fluid (CSF), neuroimaging and neurophysiology [201].

In this thesis, I focus on two autoimmune diseases of the CNS, namely MS and NMO.

\subsubsection{Multiple sclerosis}

Multiple sclerosis (MS) is the most frequent autoimmune demyelinating disease of the CNS, which is characterized by myelin destruction and infiltration of lymphocytes and monocytes across the BBB [202].

Transient neurological deficits and discrete inflammatory events (relapse) occur in 80 to $90 \%$ of patients in the early phase of the disease. The relapse is usually followed by a period of resolution (remission). This relapsingremittent course of MS usually occurs in the second or third decade of life. Relapses are usually associated with new focal and permanent lesions detectable in magnetic resonance imaging (MRI) [202].

As the number of relapses decreases [203], many patients enter a phase with more progressive neurological dysfunctions marking the secondary progressive phase of MS [204]. Only 10 to $20 \%$ of patients do not have any relapses 


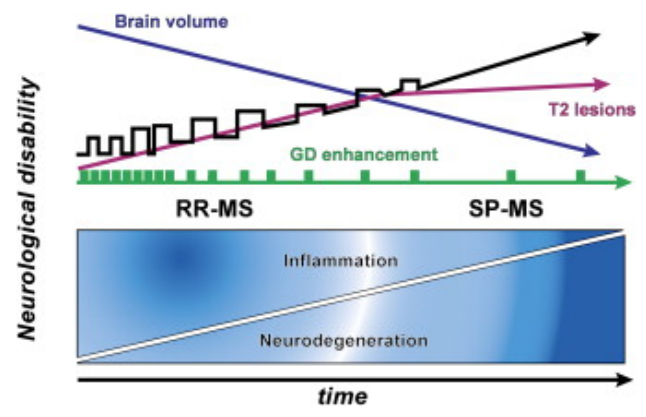

Figure 1.5: History of MS

There are different types of MS, namely the relapsing-remittent MS (RR-MS) and the progressive MS. Most patients exhibit the RR-MS with acute neurological symptoms before they convert to a secondary progressive MS (SP-MS) marked by gradual clinical worsening. The pathogenesis of MS is thought to be marked by strong initial inflammation and axonal degeneration leading to the neurodegenerative symptoms. The neurodegeneration seems to be progressive, whereas the inflammation decreases over time. Figure taken from $[202]$.

and display progressive neurological symptoms from the onset of the disease (primary progressive MS) [205] (see Figure 1.5).

Interestingly, the relapsing-remittent phase is highly variable among patients, although the progressive MS seems to progress similarly in different patients [206]. It was shown that the neurological dysfunction of the patients does not correlate with the inflammation, but rather with the accumulation of the axonal damage upon progression of the disease [207]. Axonal transsection was suggested to occur early in disease course [208] [209] [207] and seems to be directly mediated by inflammatory cells and their toxic cytokines.

\subsubsection{Pathology of Multiple sclerosis}

MS is presenting frequently with multiple white matter lesions at different locations - preferentially in the corpus callosum (CC) and the deep periventricular regions in the brain, but also in the spinal cord [202]. The lesion sites are marked by a disrupted BBB and perivenous inflammation with lymphocytes, astrocytes, activated microglia [210]. The tissue scar around the lesions was shown to be mainly composed of astrocytes, but also activated microglia and ODC's. Astrogliosis, axonal loss and global brain atrophy are the hallmarks of MS and its disease models [211] [212].

Due to the diversity of lesion pathology, Lucchinetti and colleagues characterized four lesion patterns based on complement activation, immunoglobulin deposition and MAG staining. Demyelinating lesions type I are marked by T cell- and macrophage-mediated demyelination without complement or immunoglobulin deposits, whereas the most common lesion pattern 
II shows antibody and complement deposition. Lesions of type III pattern are characterized by apoptotic ODC's, as well as signs of $\mathrm{T}$ cell, microglia and macrophages infiltration [213]. The loss of MAG and CNP staining in these lesions further indicates stressed ODC that are unable to support their distal processes [201]. Pattern IV lesions are marked by primary ODC damage and secondary demyelination that only occurs in a subset of patients with primary progressive MS and virus-mediated animal models [213].

Lesion heterogeneity seems to result from different pathogenic mechanisms and cannot be seen as a marker for different stages of the disease [214]. Until today, the complex mechanism of lesion formation is not unraveled, but it is known that $\mathrm{T}$ cells, myelin antibodies, complement and toxic macrophage products play a role [212].

Cortical demyelination and diffuse white matter lesions are more prominent in the progressive MS disease course; whereas for the relapsing-remittent phase, lesions are spread throughout the brain with new, focal inflammatory lesions [215]. The normal appearing white matter (NAWM) of the brain was also found to be altered in MRI and seems to accumulate axonal injury progressively [216]. Furthermore, cortical plaques were shown to contribute to neurological symptoms involving motor and sensory dysfunction, but also cognitive problems [217] [218].

In EM, MS lesions display complete demyelination with axonal preservation in the center of the lesions, whereas at the border of the lesions the myelin sheaths were found intact. At the rim of the lesions, some myelin sheaths were found to be split or contains vesicles at the inner tongue, but degenerated myelin sheaths were not observed frequently [219] [220].

\subsubsection{Immune cells in Muliple Sclerosis}

Various immune cells infiltrate MS lesions, such as T cells, macrophages, microglia and astrocytes and there is a complex interplay between these cells. In the blood and the CSF of MS patients, myelin-reactive T cells are present with an enhanced activation status [221] [222] [223] [224]. CD8 ${ }^{+}$ T cells clonally expand in the CSF [225] [226], can invade the CNS and encounter their antigen in an MHC class I context on glia [227] or neurons [228] [229]. These CD ${ }^{+} \mathrm{T}$ cells mediate lysis of these cell by release of cytotoxic proteins, such as perforins, granzymes and the Fas ligand. In animal models of MS, $\mathrm{CD} 8^{+} \mathrm{T}$ cells mediated progressive disease courses with demyelination and vast cell death around the blood vessels [230]. CD4 ${ }^{+} \mathrm{T}$ cells generate pro-inflammatory cyto- and chemokines that lead to the activation of microglia and the recruitment of peripheral macrophages to the site of inflammation [231] [232]. Inhibition the $\mathrm{CD} 4^{+} \mathrm{T}$ cell response was either inefficient or even worsened the course of the disease [233]. 
Microglia and macrophages on the other hand release several factors contributing to demyelination and further inflammation, such as the cytokines tumor necrosis factor $\alpha$, Fas ligand and proteases, reactive oxygen species and glutamate [202] [234] [235]. Astrocytes release a set of pro-inflammatory cytokines (tumor necrosis factor $\alpha$ and interleukin $1 \beta$ ) and prostaglandins [236] [237] and synthesize glycosaminoglycan hyaluron accumulating in the lesion that inhibits OPC proliferation [238]. Albeit their detrimental effect in promoting inflammation, destruction of ODC's and axons, astrocytes are also important for generating a permissive environment by forming a glial scar that allows repair, OPC differentiation and remyelination.

In order to develop strong autoimmune reactions, lymphocytes need to escape the control of the immune system to eliminate self-reactive lymphocytes by clonal deletion or anergy. Furthermore, activation of auto-reactive lymphocytes needs to occur and molecular mimicry or epitope spreading are discussed as potential mechanisms for initiation of autoimmunity (reviewed in [239]): A misguided immune response by molecular mimicry can be triggered by a pathogen infection with sufficient homology to autoantigens [240] [241] [242]. Epitope spreading could be elicited by bystander activation of auto-reactive $\mathrm{T}$ cells in the infected organ with pro-inflammatory environment [243] [244].

Despite intensive research, the mechanism for the pathogenesis of the different types of MS is not elucidated until today. It was even proposed that MS is a primary cytodegenerating disease that persists subclinically for prolonged time until the overt immune response of the patients is elicited as a secondary effect [220].

\subsubsection{Animal models of Multiple Sclerosis}

In order to mimic the different aspects of MS, a variety of animal models were developed. These can be categorized in virus-, toxin- or autoimmunemediated models for demyelination. Virus-mediated models are induced by neurotropic viruses, such as Semliki Forest virus, Theiler's murine encephalomyelitis virus and the John Cunningham virus. These viruses induce chronic demyelinating lesions either by oligodendropathy or by activation of the humoral response consistent via molecular mimicry or epitope spreading [245] [246]. The second group of demyelinating animal models are mediated by toxins, like the bioactive lipid lysolecithin and the copper chelator cuprizone (bis-(cyclo-hexanone)-oxaldihydrazone). The last group models of animals models mimic the autoimmune aspects of the disease by immunization of susceptible animals, such as mice, rats or non-human primates with myelin antigens, e.g. myelin, spinal cord homogenate or MOG protein or peptides together with complete Freund's adjuvants [247]. In the next 
paragraphs, the different animal models are presented in more details.

\subsection{Lysolecithin model}

Focal injection of the detergent-like lipid lysophosphatidylcholine (lysolecithin) in the spinal cord or cerebellar peduncle is part of the toxic, nonimmune mediated models resulting in focal demyelination with mild axonal loss [248] [249]. The lesions are marked by astrogliosis, as well as infiltration and activation of macrophages and microglia [250].

This demyelinating model is reversible, as remyelination starts within one week after injection in mice and the lesion is capable of complete remyelination due to OPC proliferation and differentiation [251]. Lysolecithin can also be used as an in vitro model by employing organotypic cerebellar brain slices. In this model, the expression of myelin proteins decrease before demyelination occurred after about 15 hours [252].

It is suggested that lysolecithin acts like a membrane-solubilizing agent by first targeting the myelin sheath. The degradation of myelin eventually leads to demise of ODC's [249]. Furthermore, lysolecithin activates mitogenactivated protein kinase (MAPK) pathway, as well as protein kinase $\mathrm{C}$ that further contribute to the apoptosis of myelinating cells [253] [254].

\subsection{Cuprizone model}

The cuprizone model for demyelination involves the oral administration of cuprizone that leads to highly reproducible demyelination in different brain regions, such as $\mathrm{CC}$ and the superior cerebellar peduncle [255], but also to lower extent in the cortex of mice [256]. The mechanism of action has not been elucidated. However, cuprizone is copper chelator that decreases mitochondrial respiration due to the inhibition of the copperdependent mitochondrial enzymes, cytochrome oxidase and monoaminase oxidase [255] [257]. Interestingly, the effect of cuprizone is not prevented upon co-administration of copper [258] and cuprizone has no direct effect on ODC's [259]. Nevertheless, it has a toxic effect on ODC's in vivo and on the liver of mice [259].

Acute demyelination is achieved by feeding susceptible mice with $0.2 \%$ $(\mathrm{w} / \mathrm{w})$ cuprizone in chow for at least 3 weeks [260]. After exchanging the food for normal chow, remyelination was observed. Chronic demyelination results from prolonged cuprizone treatment of up to 12 weeks. The susceptibility of animals towards cuprizone depends on the strain [261], age [211] and gender [262].

\subsection{Histopathology of cuprizone-treated mice}

The demyelinating areas are first observed in the caudal CC, before more 
frontal regions were affected. Before extensive demyelination and axonal loss [263] are observed, pronounced ODC apoptosis is evident that leads to severe astro- and microgliosis [264] (see Figure 1.6). The pathology presents with a lack of $\mathrm{T}$ cell infiltration and inflammed blood vessels, whereas BBB remained intact [255]. In line with that, immunodeficient RAG-deficient mice lacking $\mathrm{B}$ and $\mathrm{T}$ cells were equally affected by cuprizone administration as the wild type [255]. Albeit, inhibition of microglial activation (usually starting at 2 weeks of cuprizone exposure) using minocycline suggests their active involvement in ODC apoptosis and subsequent demyelination [259].

Oligodendropathology results in $80 \%$ decrease of expression of the myelin genes MBP, MAG and ceramide galactosyltransferase after three weeks of cuprizone exposure [266]. The stress-related protein p8 that is specific to ODC's is increased and its knock-out is less vulnerable to cuprizone treatment [267]. The severe perturbation of ODC's upon cuprizone treatment leads to demyelination without further exposure to cuprizone three weeks later [260]. Remyelination starts at peak of demyelination and removal of the toxin exacerbates the remyelination within a few weeks [268] [255] with an increased expression of myelin proteins, especially proteins related to mitochondrial function [269].

Ludwin and colleagues [270] suggest that cuprizone induces a dying-back pathology as degenerating ODC's with enlarged mitochondria were detected in EM already after one week of cuprizone exposure [271] and subsequently, two to three weeks later, demyelination was apparent [268]. Observed changes in the ultrastructure of myelin included vesiculated tubular material in the enlarged inner tongue and vacuolization of the myelin sheaths [268] [272]. At later time points, microglia were engulfing the vacuolated myelin [272].

The finding in EM can be correlated with MRI observations as the myelinated fraction in EM correlates T1-weighted images obtained from cuprizone treated mice. The magnetization transfer ratio correlates with MBP histology that is decreasing over cuprizone treatment in the CC. The bound pool fraction and the axial diffusivity correlates with the myelin sheath and the non-myelinated fraction respectively. Tissue structure changes are indicated by longitudinal relaxation rates and diffusivity measurements [273]. These findings make pharmaceutical treatment trails possible as the ultrastructural changes can be - at least in part - be deduced from MRI of treated mice.

\subsection{Experimental autoimmune encephalomyelitis}

Experimental autoimmune encephalomyelitis (EAE) is a model for the autoimmune aspects of MS due to inflammatory lesions with variable degree of 


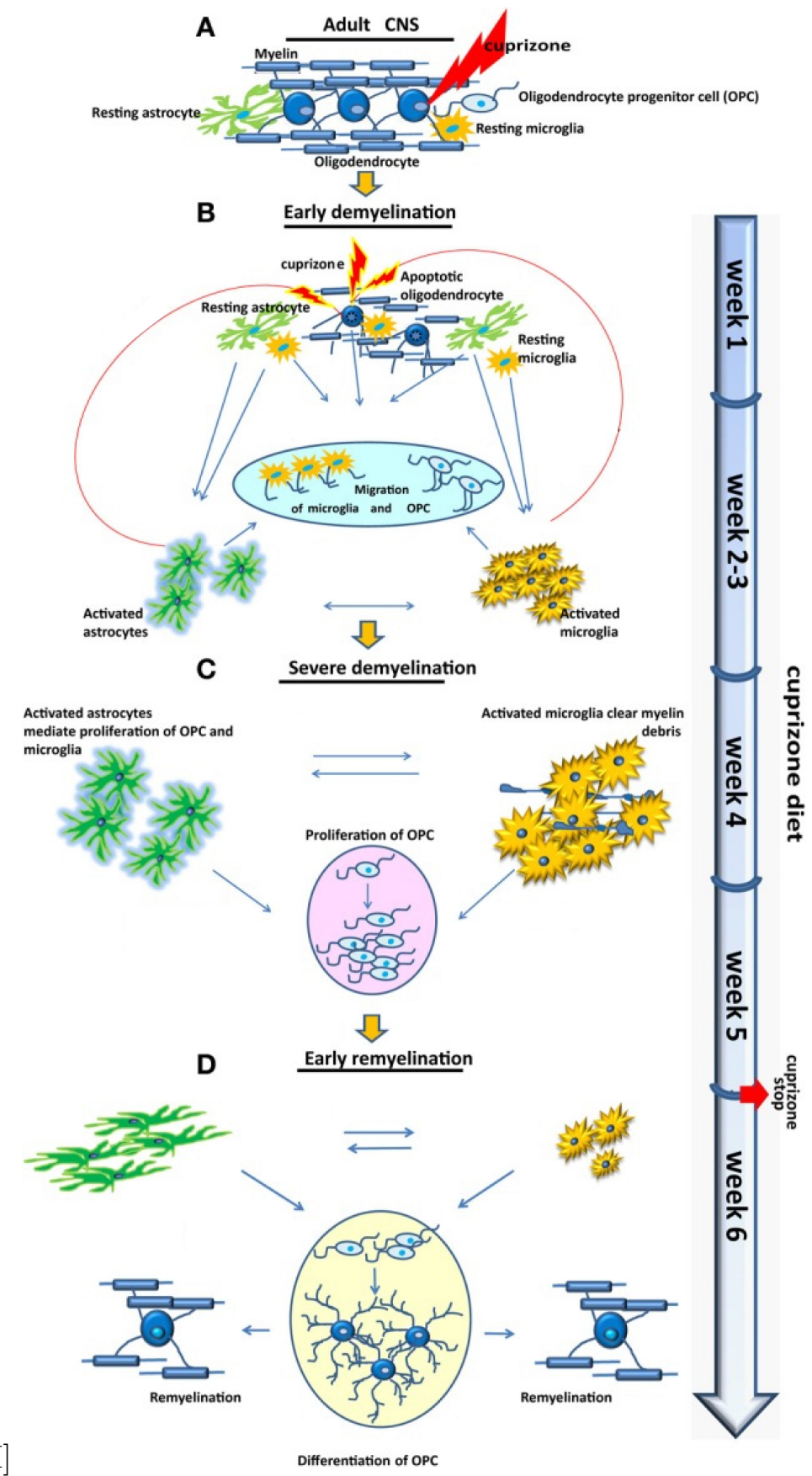

Figure 1.6: Molecular and cellular changes upon cuprizone treatment The glia cell distribution in a healthy brain is depicted in A. Upon short cuprizone treatment (B), oligodendrocytes apoptosis is induced leading to an activation of microglia and astrocytes. Their cytokines lead to migration and activation of microglia and oligodendrocyte precursor cells (OPC). C displays severe demyelination and activated microglia clearing myelin debris and OPC proliferation upon astrocyte activation. In D, OPC migrated and differentiated to initiate remyelination. Activated microglia are decreased, whereas astrocytes stay activated despite changes in their morphology. Figure adapted from [265]. 
demyelination. This model can be induced either actively via immunization of rodents or primates with myelin antigens emulsified in Freund's adjuvants or passively due to transfer of isolated myelin-reactive $\mathrm{T}$ cells into naïve animals [274] [275]. The immunization leads to neurological symptoms that can be used to track the disease course over time (see Figure 1.7). Depending on the induction protocol and animals employed, EAE can follow different clinical courses. Some mouse strains are even resistant to certain immunization protocols, whereas others exhibit a relapsing EAE.

EAE lesions are usually characterized by mononuclear infiltrates, demyelination and axonal loss, that is thought to be the underlying cause for the neurological symptoms. Although the lesions are very heterogenous, perivascular inflammation due to BBB dysfunction and demyelination are a prominent features. Furthermore, several microglia and macrophages clear the debris caused by demyelination [276] [277]. Due to the infiltration of these immune cells that seem to be mainly mediated by $\mathrm{CD} 4^{+} \mathrm{T}$ cells and macrophages, EAE lesion resemble type I or II lesion patterns in MS [213].

Irrespective of their antigen specificity, highly active lymphocytes can cross the BBB and encounter their antigens in the CNS [278]. EAE is mainly mediated by $\mathrm{CD} 4^{+} \mathrm{T}$ cells that recognise their antigen on MHC class IIpresenting microglia, resulting in their reactivation and release of their proinflammatory cytokines (Interferon $\gamma$, Interleukin 2, lymphotoxin and tumor necrosis factor $\alpha$ [279]). T cell-mediated activation of macrophages are thought be the underlying cause for inflammatory demyelination in EAE [280] as association of macrophages with the node of Ranvier were shown to induced demyelination. Interestingly, microglia were not actively participating in the demyelination, but rather clearing the myelin debris. However, Ransohoff and co-workers suggest that the role of $\mathrm{CD} 4^{+} \mathrm{T}$ cells in MS is overestimated when EAE is applied as animal model, as B and CD8 ${ }^{+} \mathrm{T}$ cells show only minor involvement in EAE. Contrarily to what is observed in MS [281].

in contrast to other mouse strains, Biozzi antibody high (ABH) mice exhibit a chronic relapsing disease course and lesions that are comparable to MS patients. EAE-inducing antigens are PLP, MOG, MBP, MAG and $\alpha \mathrm{B}-$ crystallin [283] [284] [285] [286], but the chronic relapsing disease is only triggered upon induction with spinal cord homogenate, MOG peptide aa 821 or PLP peptide aa 56-70 [283].

The acute phase is marked by intensive mononuclear cell infiltration and only a minor degree of demyelination, whereas in remission phases, the inflammatory load is reduced and white matter damage occurs [283]. The lesions also contain immunoglobulin deposits and their infiltrates consist of macrophages and to a lower degree of $\mathrm{CD} 4^{+} \mathrm{T}$ cells. Upon chronic relapsing EAE, lesions further acquire widespread demyelination, astrogliosis 


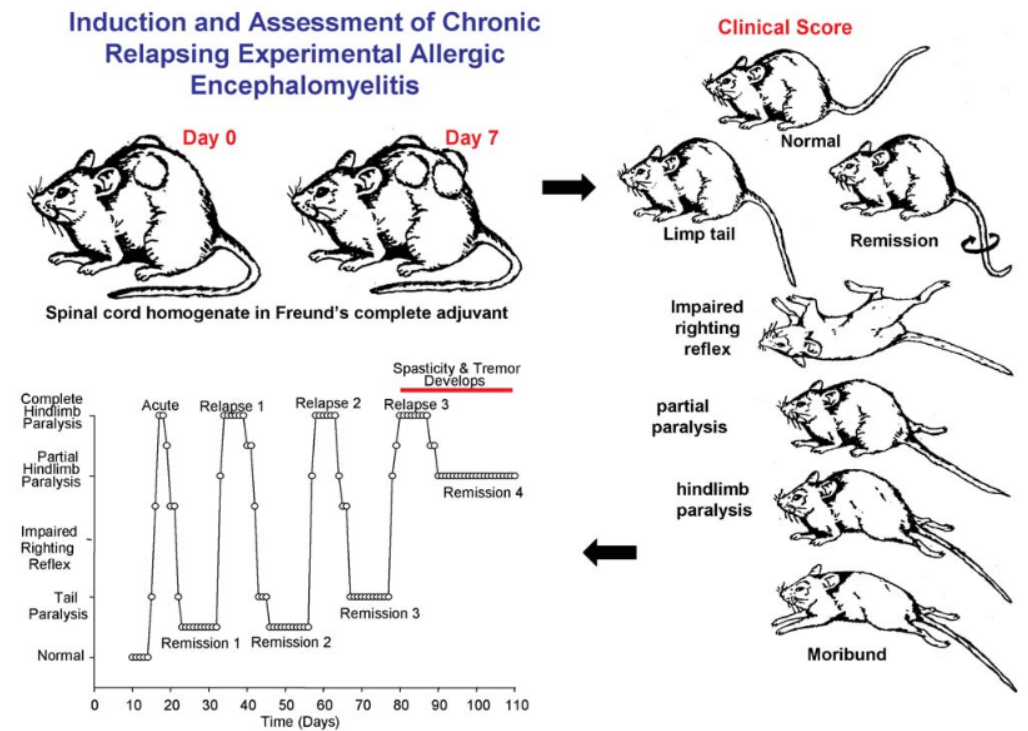

Figure 1.7: Induction and disease course of experimental autoimmune encephalomyelitis Experimental autoimmune encephalomyelitis (EAE) can be induced by spinal cord homogenate emulsified in complete Freund's adjuvants at d0 and d7. This initiates a relapsingremittent disease course in Biozzi mice leading to a chronic phase in later disease stages. Clinical scoring depicted on the right side is employed to evaluate the different phases of the disease as shown in the graph. Figure taken from [282].

and fibrosis of the blood vessel [287]. Baker et al. showed demyelination and reduced axonal transport that is thought to be a result of opsonisation of myelin with antibodies and complement [288] [289] in addition to the the receptor-mediated phagocytosis of macrophages [290]. The importance of antibodies for the development of the disease is well characterized for Biozzi mice as the same strain with low antibody titer is less susceptible to EAE [283]. This assumption is further supported by the finding that antibodies, especially against MOG, in the sera of Biozzi mice after the acute phase are present in high titer. Additionally, the monoclonal MOG antibody was found to augment EAE duration and severity resulting in large demyelinating areas in Biozzi [291] and rats [292].

\subsubsection{Neuromyelitis optica}

Neuromyelitis optica (NMO) or Devic's disease is a chronic, mostly relapsing demyelinating disease of the CNS that affect primarily the spinal cord and the optic nerves [293]. In order to diagnose this inflammatory disease, Wingerchuk et al. defined two absolute criteria, namely severe optic neuritis and extensive transverse myelitis. Furthermore, several supportive criteria were suggested: First, a brain MRI that is not meeting the criteria for the disease 
onset of MS. Second, a spinal cord MRI showing signal abnormalities in the T2-weighted images spanning over three vertebra. Finally, autoantibodies against Aquaporin 4 (AQP4) are detected in the blood [293].

The resemblance of lesions in terms of demyelination and infiltration of immune cells led to the hypothesis that NMO was a variant of MS [294] [295]. Similar to MS; complement and immunoglobulin deposits [296] [297] can be detected, as well as leucocyte, granulocyte and macrophage infiltrates due to enhanced permeability of the BBB [294] [295]. Recently, however, it was discovered that NMO is a "separate" disease with distinct pathological characteristics: NMO lesions are devoid of astrocytes and show features of apoptotic ODC's and axonal loss.

In comparison to MS, NMO patients do not display cortical demyelination, but have a complete destruction of astrocytes (or they only present with few, short processes) resulting in a reduced AQP4 staining in histology. In addition, NMO patients show perivascular inflammation with eosinophils and macrophage clusters around thickened blood vessels [201]. Although MS and NMO have very similar pathological features, it is crucial to distinguish them as their treatments are different and can even be detrimental in case of misdiagnosis. For example, Interferon $\beta$ that is beneficial in MS, was shown to increase the relapse rate in NMO [298].

One specific and sensitive biomarker is the AQP4 antibody in the CSF and the blood of the NMO patients [299] [300] [301]. AQP4 is a water channel expressed in the end feet of astrocytic processes abutting capillaries of the $\mathrm{BBB}[300]$ and the serum titer of the antibody was found to correlate with the disease severity of NMO [302] [303]. Staining with an AQP4 antibody in healthy CNS tissue shows rims or rosettes similar to immunoglobulin and complement deposition in NMO lesions [304]. Hence, it was suggested that NMO is a astrocytopathic disease caused by the pathogenic AQP4 antibody. The lesion development depends on AQP4 as AQP4-deficient mice did not show any lesions upon treatment of spinal cord slices with the pathogenic AQP4 antibody. Furthermore, it was shown that macrophages, activated microglia and their cytokines exacerbate the effect of the antibody when applied on astrocytes in spinal cord slice culture at lower concentrations [305].

In animal models of NMO, the AQP4 antibody exacerbates lesions of rats induced with EAE [301] [306] [307] or rats treated with CFA alone [308]. Intracranial injection of the antibody in naïve mice or rats resulted in NMOlike lesions with demyelination, astrocytopathy, complement deposition and infiltration of CD45 ${ }^{+}$cells [309] [310] [307]. Depletion of astrocytes was shown to be complement dependent and antibody mediated resulting in secondary demyelination and inflammation upon apoptosis of ODC's [307] (see 1.8). 
The mechanisms leading to the observed demyelination is not yet understood, but thought at least in part to be mediated by excitotoxicity of glutamate [311] [312]. Astrocytes mediate not only the water homeostasis, but are also responsible for removal of the neurotransmitter glutamate via the excitatory amino acid transporter 2 (EAAT2) [312]. Hinson and colleagues could show the internalization of the glutamate transporter EAAT2 with AQP4 upon treatment of cells with the AQP4 antibody and complement. Thus, they suggested that the EAAT2 known to be critical for sodium-dependent glutamate clearance from excitatory synapses [313] might lead to a disruption in glutamate levels and hence, to excitotoxicity of ODC's upon AQP4 antibody treatment of astrocytes [314] [312]. The pathological mechanism induced by AQP4 antibodies proposed by Hinson et al. is criticized as the internalization of the heterotetramer formed by $\mathrm{AQP} 4$ and the decreased water permeability, as well as the independence on complement could not be reproduced by other groups [315] [316] [317].

Despite the fact that the AQP4 antibody was shown to mediate NMO, there is a subset of patients that do not have the AQP4 antibody, but are seropositive for a MOG antibody and they have a better prognosis. NMO lesions of these patients display demyelination, but no astrocytic damage [318]. The molecular mechanism of the MOG antibody-mediated demyelination remains to be elucidated. 


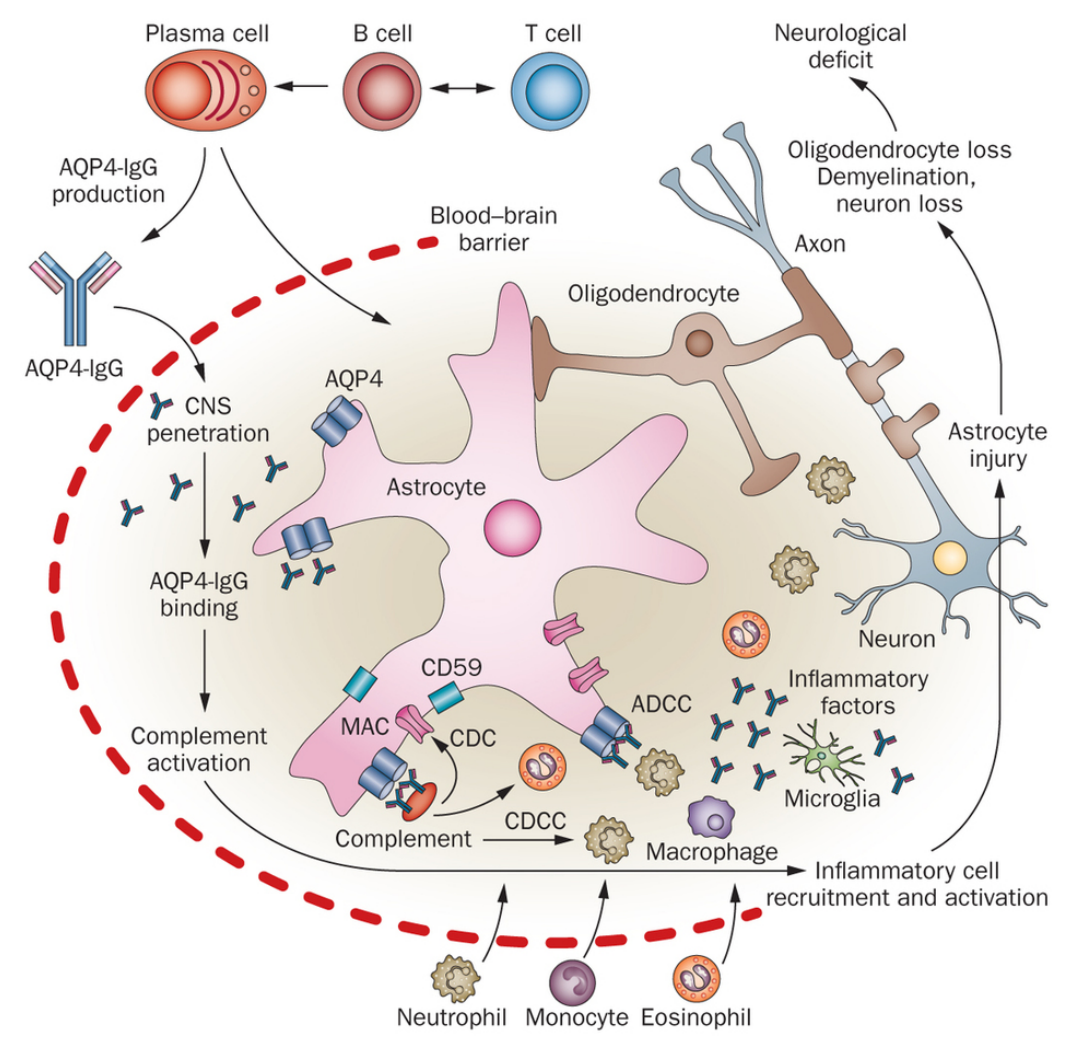

Figure 1.8: Suggested mechanism for NMO pathology

After the AQP4 antibody crosses the blood brain barrier, it can bind to AQP4 water channels on the astrocyte feet mediating antibody and complement-dependent cytotoxicity. The complement activation leads to recruitment of peripheral immune cells, oligodendrocyte injury, demyelination and neuronal loss. Abbreviations: ADCC, antibodydependent cellular cytotoxicity; AQP4, aquaporin 4; CDC, complement-dependent cytotoxicity; CDCC, complement-dependent cellular cytotoxicity; MAC, membrane attack complex; NMO, neuromyelitis optica. Figure taken from [319]. 


\subsection{Project aim}

Myelin breakdown is a pathological hallmark of several autoimmune diseases in the nervous system. MBP is so far the only known protein essential for myelin assembly [88]. Hence, I aimed to elucidate whether MBP plays a role in the underlying mechanism of myelin fragmentation in demyelinating diseases.

In this thesis, I aim to investigate whether a loss of MBP function is the key event initiating myelin fragmentation in vivo. As naturally unstructured protein with a high positive charge at physiological $\mathrm{pH}$ and very little secondary structure, the binding of MBP to negatively charged lipids leads to charge neutralization and allows it to self-interact. This leads to a transition of a soluble phase of free MBP molecules to a condensed phase formed by the cohesive network of MBP. The reversal of this transition of MBP from the membrane-bound phase to a dispersed state might in turn have a big impact on the ultrastructure of myelin leading to its collapse.

The detailed aims were:

1.) to perform an ultrastructural analysis of demyelinating models In particular, toxin- and immune-mediated models of demyelination in mice and rats were assessed. Time course studies of the demyelinating conditions were prepared by state-of-the-art sample preparation techniques for EM to ensure the best possible myelin preservation. Here, the myelin fragmentation profiles were assessed for the different demyelinating models.

2.) to identify tools to study reverse phase transition of MBP

MBP is responsible for the compaction of the membrane and according to our hypothesis, MBP is rendered dysfunctional leading to the initiation of myelin fragmentation. Hence, we needed to identify tools to study the reverse phase transition in vitro and in vivo.

\section{3.) to characterize the tools detecting dysfunctional MBP using} ODC cultures

In order to confirm our hypothesis and to characterize the tool established in 2., we needed to perform treatments of ODC that are known to cause a disruption of the MBP network. With the help of these treatments, the pool of MBP detected by these tools needed to be investigated in term of membrane binding, self-interaction and extrusion function.

4.) to apply this tool to early time points of demyelinating animal models

To assess whether the tools can also be used in immunohistochemistry and to test our hypothesis of reverse phase transition as key event for myelin disassembly, we stained early time points of demyelinating animal models where myelin pathology was present as confirmed in 1.

5.) to elucidate the mechanism that triggers reverse phase trans- 


\section{ition of MBP}

In the end, we aimed to further investigate the mechanism that leads to reverse phase transition of MBP and to determine, if this effect can be prevented by blocking the trigger for dysfunctional MBP.

Hence, in this thesis I investigate on whether changes in MBP can affect myelin stability and initiate myelin fragmentation in vivo. 


\section{Chapter 2}

\section{Material and Methods}

"Theory guides.

Experiment decides."

Izaak Kolthoff

\subsection{Material}

\subsubsection{Chemicals and consumables}

Chemicals used in this study were obtained from Sigma-Aldrich (SigmaAldrich, Munich, Germany), Merck (Merck, Darmstadt, Germany) or AppliChem (AppliChem, Darmstadt, Germany) unless stated otherwise. Lipids used were obtained from Avanti Polar Lipids (Alabaster, USA) as chloroform stocks. All basal media, supplements, antibiotics and sera for cell culture were purchased from Gibco/Invitrogen (Invitrogen, Darmstadt, Germany) unless specified. Dulbecco's Modified Eagle Medium (DMEM) with 4,5 g/L glucose, horse serum (HS), fetal calf serum (FCS) and phosphate buffered saline (PBS) were obtained from PAA (Cölbe, Germany).

Consumables were purchased from Falcon (Becton Dickinson, Le Pont De Claix, France) and Eppendorf (Eppendorf, Hamburg, Germany). Culture vessels were obtained from Greiner Bio-One (Greiner Bio-One, Frickenhausen, Germany).

\subsubsection{Antibodies used in this thesis}

Primary antibodies used in this thesis are listed in Table 2.1. Secondary antibodies were purchased from Dianova (Hamburg, Germany) and usually 
used 1:500 for immunohistological studies, whereas the horse radish peroxidase conjugates secondary antibodies used for Western Blotting were diluted $1: 2000$.

Table 2.1: Antibodies used in this thesis

\begin{tabular}{|c|c|c|c|}
\hline Antigen & Host species & Application & Manufacturer \\
\hline Actin (AC-40) & mouse IgG2a & WB (1:500) & $\begin{array}{l}\text { Sigma-Aldrich, } \mathrm{Mu}- \\
\text { nich. Germany }\end{array}$ \\
\hline APP & mouse IgG & IHC $(1: 100)$ & $\begin{array}{l}\text { Chemicon, Billerica, } \\
\text { USA }\end{array}$ \\
\hline Calnexin & rabbit & WB $(1: 1000)$ & $\begin{array}{lr}\text { Stressgen } & \text { Biore- } \\
\text { agent, } & \text { Victoria, } \\
\text { Canada } & \end{array}$ \\
\hline CD3 & rat & IHC (1:300) & $\begin{array}{l}\text { Serotec, Puchheim, } \\
\text { Germany }\end{array}$ \\
\hline CNP & mouse & IF $(1: 300)$ & $\begin{array}{l}\text { Sigma-Aldrich, Mu- } \\
\text { nich, Germany }\end{array}$ \\
\hline CNP & rabbit & IF $(1: 300)$ & $\begin{array}{l}\text { Sigma-Aldrich, Mu- } \\
\text { nich, Germany }\end{array}$ \\
\hline $\begin{array}{ll}\text { GalC } & \text { (clone } \\
\text { MAB342) }\end{array}$ & mouse IgG1 & IF $(1: 200)$ & $\begin{array}{l}\text { Millipore, Billerica, } \\
\text { USA }\end{array}$ \\
\hline $\mathrm{HA}$ & rabbit & IF $(1: 500)$ & $\begin{array}{l}\text { Abcam, Cambridge, } \\
\text { UK }\end{array}$ \\
\hline Iba1 & rabbit & IHC $(1: 200)$ & $\begin{array}{l}\text { Wako Chem., Neuss, } \\
\text { Germany }\end{array}$ \\
\hline Mac3 & rat & $\operatorname{IHC}(1: 500)$ & $\begin{array}{l}\text { Pharmingen, } \\
\text { Diego, USA }\end{array}$ \\
\hline MBP & rabbit & $\begin{array}{l}\mathrm{IF} / \mathrm{IHC}(1: 300), \mathrm{WB} \\
(1: 5000)\end{array}$ & $\begin{array}{l}\text { Dako Cytomat., } \\
\text { Carpinteria, USA }\end{array}$ \\
\hline MBP & mouse IgG1 & IF $(1: 1000)$ & $\begin{array}{l}\text { Sternberger, } \\
\text { Lutherville, USA }\end{array}$ \\
\hline $\begin{array}{l}\text { MOG (clone 8-18- } \\
\text { C5) }\end{array}$ & mouse IgG1 & $\begin{array}{l}\text { IF }(1: 100), \quad \text { WB } \\
(1: 1000)\end{array}$ & $\begin{array}{l}\text { Millipore, Billerica, } \\
\text { USA }\end{array}$ \\
\hline Мyc & rabbit IgG & IF $(1: 500)$ & $\begin{array}{l}\text { Upstate, Lake Pla- } \\
\text { cid, USA }\end{array}$ \\
\hline $\mathrm{O} 1$ & mouse IgM & IF $(1: 50)$ & $\begin{array}{l}\text { self-made from hy- } \\
\text { bridoma [320][321] }\end{array}$ \\
\hline $\mathrm{O} 4$ & mouse IgM & IF $(1: 50)$ & $\begin{array}{ll}\text { self-made from } \\
\text { hybridoma[322][320] }\end{array}$ \\
\hline PIP2 & mouse IgG2b & IF/IHC (1:200) & $\begin{array}{l}\text { Echelon Biosciences, } \\
\text { Salt Lake City, USA }\end{array}$ \\
\hline QD9 & mouse & $\begin{array}{l}\operatorname{IF} \quad(1: 1000), \quad \text { IHC } \\
(1: 2000)\end{array}$ & $\begin{array}{l}\text { Abcam, Cambridge, } \\
\text { UK }\end{array}$ \\
\hline Tubulin, $\alpha$ or $\beta$ & mouse IgG1 & $\begin{array}{l}\text { IF } \quad(1: 2000), \quad \text { WB } \\
(1: 5000)\end{array}$ & $\begin{array}{l}\text { Sigma-Aldrich, Mu- } \\
\text { nich, Germany }\end{array}$ \\
\hline Tubulin, $\beta$ III & mouse IgG1 & IF $(1: 3000)$ & $\begin{array}{l}\text { Promega, Man- } \\
\text { nheim, Germany }\end{array}$ \\
\hline
\end{tabular}

\subsubsection{Kits used}

Kits used in this study are listed in Table 2.2 and were used according to the manufacturer's guidelines. 
Table 2.2: Commercial kits

\begin{tabular}{|c|c|c|}
\hline Commercial kit & Application & Manufacturer \\
\hline NucleoSpin Plasmid kit & DNA isolation, small scale & $\begin{array}{l}\text { Macherey-Nagel, } \\
\text { Germany }\end{array}$ \\
\hline NucleoBond Xtra Midi kit & DNA isolation, medium scale & $\begin{array}{l}\text { Macherey-Nagel, } \\
\text { Germany }\end{array}$ \\
\hline $\begin{array}{l}\text { NucleoSpin Gel and PCR } \\
\text { Clean-up }\end{array}$ & $\begin{array}{l}\text { DNA extraction from agarose } \\
\text { gels }\end{array}$ & $\begin{array}{l}\text { Macherey-Nagel, Düren, } \\
\text { Germany }\end{array}$ \\
\hline Lipofectamine 2000 & transient transfection & $\begin{array}{l}\text { Invitrogen, Darmstadt, Ger- } \\
\text { many }\end{array}$ \\
\hline Pierce BCA protein assay & protein quantification & $\begin{array}{l}\text { Thermo Scientific, Waltham, } \\
\text { USA }\end{array}$ \\
\hline Pierce Direct IP kit & Immunoprecipitation & $\begin{array}{l}\text { Thermo Scientific, Waltham, } \\
\text { USA }\end{array}$ \\
\hline $\begin{array}{l}\text { SuperScript III First-Str. } \\
\text { Synth. }\end{array}$ & cDNA synthesis & $\begin{array}{l}\text { Invitrogen, Darmstadt, Ger- } \\
\text { many }\end{array}$ \\
\hline
\end{tabular}

\subsubsection{Enzymes used in this studies}

Table 2.3: Enzymes used in this study

\begin{tabular}{l|l|l} 
Enzyme & Application & Manufacturer \\
\hline $\begin{array}{l}\text { Restriction enzymes } \\
\text { T4 DNA ligase }\end{array}$ & $\begin{array}{l}\text { DNA digest } \\
\text { Ligation of DNA fragments } \\
\text { Taq DNA polymerase }\end{array}$ & $\begin{array}{l}\text { NEB or Fermentas } \\
\text { Fermentas, Waltham, USA } \\
\text { Promega, Mannheim, Ger- } \\
\text { many }\end{array}$ \\
Phusion DNA polymerase & Polymerase chain reaction & $\begin{array}{l}\text { Thermo Scientific, Waltham, } \\
\text { USA }\end{array}$ \\
Antartic phosphatase & 5'-phosphate removal & NEB, Frankfurt, Germany \\
T4 polynucleotide kinase & 5 -phosphorylation & NEB, Frankfurt, Germany
\end{tabular}

\subsubsection{General Buffers and Solutions}

\section{Phosphate Buffered Saline (PBS)}

10X PBS was prepared as follows:

$10 X P B S(1 L)$

$80.0 \mathrm{~g} \mathrm{NaCl}$

$2.0 \mathrm{~g} \mathrm{KCl}$

$14.4 \mathrm{~g} \mathrm{Na}_{2} \mathrm{HPO}_{4}$ (or $18.05 \mathrm{~g} \mathrm{Na} 2 \mathrm{HPO}_{4} \cdot 2 \mathrm{H}_{2} \mathrm{O}$ )

$2.4 \mathrm{~g} \mathrm{KH}_{2} \mathrm{PO}_{4}$

To obtain 1X PBS, 10X PBS was diluted 10 times with $\mathrm{ddH}_{2} \mathrm{O}, \mathrm{pH}$ value was adjusted to 7.4 and stored at RT.

\section{Krebs-Ringer solution}

Krebs-Ringer solution (KR) was obtained by diluting the following chemicals in $\mathrm{ddH}_{2} \mathrm{O}$ : 
Krebs-Ringer solution ( $50 \mathrm{~mL})$

$0.29 \mathrm{~g} \mathrm{NaCl}$

$17.39 \mathrm{mg} \mathrm{KCl}$

$90 \mathrm{mg}$ glucose

$238 \mathrm{mg}$ HEPES

The volume was added to $40 \mathrm{~mL}$, then the $\mathrm{pH}$ was adjusted to 7.4 and subsequently, the following solutions were added:

$120 \mu \mathrm{L} 0.5 \mathrm{M} \mathrm{CaCL}_{2}$

$70 \mu \mathrm{L} 0.5 \mathrm{M} \mathrm{MgSO}_{4}$ The volume was added up to $50 \mathrm{~mL}$ and the solution was stored at $4^{\circ} \mathrm{C}$ for 2 weeks at most.

\section{Paraformaldehyde (PFA) solution for fixation}

Paraformaldehyde (PFA) 16\% stock solution was prepared by dissolving $16 \mathrm{~g}$ PFA in $70 \mathrm{~mL}$ water, heating at $60^{\circ} \mathrm{C}$ and adding $\mathrm{NaOH}$ pellets until the solution became clear. Then, $10 \mathrm{~mL} 10 \mathrm{X}$ PBS were added and $\mathrm{pH}$ was adjusted to 7.4. After adjusting the volume with water to $100 \mathrm{~mL}$, the solution was aliquoted and stored at $-20^{\circ} \mathrm{C}$. Working solution of $4 \% \mathrm{PFA}$ in PBS was prepared freshly by diluting $50 \mathrm{~mL}$ of PFA stock solution in $150 \mathrm{~mL}$ of 1X PBS and stored at $4^{\circ} \mathrm{C}$ for at most a week.

\section{Blocking solution for immunocytochemistry}

The stock blocking solution (100\%) was obtained by diluting the following reagents in $100 \mathrm{~mL} 1 \mathrm{X}$ PBS:

$2 \mathrm{~mL}$ FCS

$2 \mathrm{~g}$ BSA

$2 \mathrm{~g}$ fish gelatin

\section{Mowiol solution for immunocytochemistry}

Mowiol was used as the mounting medium after immunocytochemistry and immunohistochemistry. The solution was prepared by stirring $2.4 \mathrm{~g}$ mowiol, $6 \mathrm{~g}$ glycerol and $6 \mathrm{~mL}$ water for several hours at RT. After addition of $12 \mathrm{~mL}$ $0.2 \mathrm{M}$ Tris- $\mathrm{HCl}(\mathrm{pH} 8.5)$, the solution was incubated at $60^{\circ} \mathrm{C}$ for $10 \mathrm{~min}$ and then, centrifuged at $4000 \mathrm{~g}$ for $15 \mathrm{~min}$. The mowiol was aliquoted an kept at $-20^{\circ} \mathrm{C}$ until further use.

\section{X Protease Inhibitor solution (PI)}

In order to inhibit a wide range of proteases, the protease inhibitors cocktail Complete Mini (Roche Applied Science, Mannheim, Germany) was used for biochemical studies. For that purpose, one tablet was dissolved in $1 \mathrm{~mL} 1 \mathrm{X}$ PBS and aliquots were stored at $-20^{\circ} \mathrm{C}$. A 1:50 dilution of this stock was added to the lysis buffer directly before use.

\section{Lysis buffer}

$2 \mathrm{X}$ lysis buffer was prepared as follows and stored at $4^{\circ} \mathrm{C}$ : 
$2 X$ Lysis buffer $(50 \mathrm{~mL})$

$2 \mathrm{~mL}$ NP-40

$2 \mathrm{~mL} 10 \%$ SDS

$10 \mathrm{~mL} 10 \mathrm{X}$ PBS

$19.01 \mathrm{mg}$ EGTA

add to $50 \mathrm{~mL}$ with $\mathrm{ddH}_{2} \mathrm{O}$ To obtain $1 \mathrm{X}$ lysis buffer, with $\mathrm{ddH}_{2} \mathrm{O}$ with the appropriate volume after addition of 1:50 dilution of PI.

\section{Sample buffer}

$4 \mathrm{X}$ sample buffer was prepared as follows and stored at RT:

$4 X$ sample buffer $(10 \mathrm{~mL})$

$1.67 \mathrm{~mL} 1.5 \mathrm{M}$ TRIS $\mathrm{pH} 6.8$

$80 \mu \mathrm{L} 0.5 \mathrm{M}$ EDTA $\mathrm{pH} 8.0$

$0.8 \mathrm{~g}$ SDS

$2 \mathrm{~mL} 0.2 \%$ bromphenol blue

$400 \mu \mathrm{L} \beta$-mercaptoethanol

$4 \mathrm{~mL}$ glycerol

add to $10 \mathrm{~mL}$ with $\mathrm{ddH}_{2} \mathrm{O}$.

Tris Buffered Saline (TBS)

10X TBS was prepared as follows:

$10 X T B S(1 L)$

$60.6 \mathrm{~g}$ Tris

$87.6 \mathrm{~g} \mathrm{NaCl}$

To obtain $1 \mathrm{X}$ TBS, 10X TBS was diluted 10 times with $\mathrm{ddH}_{2} \mathrm{O}, \mathrm{pH}$ value was adjusted to 7.4 and the solution was stored at RT.

\section{Avertin}

Avertin was prepared by mixing the following components at $40^{\circ} \mathrm{C}$, while stirring:

1 g 2,2,2-Tribromoethanol (99\%)

$0.81 \mathrm{~mL}$ tert-amylalcohol

$71.49 \mathrm{~mL} \mathrm{H}_{2} \mathrm{O}$

The solution was filtered and stored at $4^{\circ} \mathrm{C}$ for at most one week.

\section{Anesthetic}

$4 \mathrm{mg}$ Xylazine (2\% Rompun, Bayer Vita, Leverkusen, Germanyl)

$60 \mathrm{mg}$ Ketamine (10\% Ketamine, WDT, Garbsen, Germany)

In order to anaesthetize a mouse, the body weight was determined and $6 \mu \mathrm{g}$ Ketamine and $900 \mu \mathrm{g}$ Xylazine per $10 \mathrm{~g}$ body weight were injected intraperitoneally (ip).

\section{Artifical cerebrospinal fluid (aCSF)}

Artifical cerebrospinal fluid (aCSF) was prepared as follows: 
Artifical cerebrospinal fluid (1 L)

$8.66 \mathrm{~g} \mathrm{NaCl}$

$223.6 \mathrm{mg} \mathrm{KCl}$

$155.3 \mathrm{mg} \mathrm{CaCl} \mathrm{Cl}_{2}$

$76.1 \mathrm{mg} \mathrm{MgCl}_{2}$

$113.5 \mathrm{mg} \mathrm{Na} 2 \mathrm{HPO}_{4} 4$

$23.9 \mathrm{mg} \mathrm{NaH} \mathrm{PO}_{4} 4$

The $\mathrm{pH}$ was adjusted to 7.4 before $\mathrm{ddH}_{2} \mathrm{O}$ was added to $1 \mathrm{~L}$. The solution was stored at $4^{\circ} \mathrm{C}$.

\section{Phosphate Buffer (PB)}

$0.1 \mathrm{M}$ Phosphate buffer (PB) was prepared as follows:

Phosphate buffer solution (200 $\mathrm{mL}$ )

$0.36 \mathrm{~g} \mathrm{NaH}_{2} \mathrm{PO}_{4} \cdot \mathrm{H}_{2} \mathrm{O}$

$3.1 \mathrm{~g} \mathrm{Na}_{2} \mathrm{HPO}_{4} \cdot 2 \mathrm{H}_{2} \mathrm{O}$

The volume was added up to $200 \mathrm{~mL}$ with $\mathrm{ddH}_{2} \mathrm{O}$ and the solution was stored at RT. The working solution of $\mathrm{PB}$ was obtained by diluting the stock solution 1:5 in $\mathrm{ddH}_{2} \mathrm{O}$.

\section{Epoxy resin (Epon)}

$21.4 \mathrm{~g}$ Glycid ether 100 (Serva, Heidelberg, Germany)

$14.4 \mathrm{~g}$ 2-Dodecenylsuccinic acid anhydride (DDSA) (Serva, Heidelberg, Germany)

$11.3 \mathrm{~g}$ Methylnadic anhydride (MNA) (Serva, Heidelberg, Germany)

Solution was stirred for $10 \mathrm{mins}$ and after addition of $0.84 \mathrm{~mL} \mathrm{2,4,6-Tris(di-}$ methyl-aminomethyl-)-phenol (DMP-30) (Electron Microscopy Sciences, Munich, Germany), stirred for additional 20 mins before use.

\section{Formvar solution}

$625 \mathrm{mg}$ Formvar (Plano, Wetzlar, Germany)

$50 \mathrm{~mL}$ Chloroform

Stir for at least 30 mins. Store at RT protected from light.

\section{Richardson's Methylene blue / Azure II blue}

$1 \%[\mathrm{w} / \mathrm{v}]$ Azure II (Merck, Darmstadt, Germany) in $\mathrm{ddH}_{2} \mathrm{O}$ (stock solution I)

$1 \%[\mathrm{w} / \mathrm{v}]$ Methylene Blue (Merck, Darmstadt, Germany) in 1\% sodium borate (stock solution II)

The stock solutions were stored at RT. For staining of lipid-rich regions, equal volumes of the stock solutions were mixed and filtered directly before use. 


\subsubsection{Primer}

The primers used in this studies were synthesized in the DNA core facility, the AGCT-laboratory of the Max-Planck Institute for Experimental Medicine (Göttingen, Germany).

The primers shown in Table 2.4 were used to generate mutants of MBP by site-directed mutagenesis.

Table 2.4: Primers

\begin{tabular}{|c|c|}
\hline Construct / project & 5' to 3 ' primer sequence \\
\hline $\mathrm{MBP} F 1 \rightarrow \mathrm{S}$ & $\begin{array}{l}\text { for.:GAAAACCCAGTAGTCCATTAG } \\
\text { TTCAAGAACATTGTG } \\
\text { rev.: CACAATGTTCTTGAACT } \\
\text { AATGGACTACTGGGTTTTC }\end{array}$ \\
\hline $\mathrm{MBP}$ F2,3 $\rightarrow \mathrm{S}$ & $\begin{array}{l}\text { for.: CTTGACTCCATCGGGCGCTCATC- } \\
\text { GAGCGGTGACAGGGGTG } \\
\text { rev.: CACCCCTGTCACCGCT } \\
\text { CGATGAGCGCCCGATGGAGTCAAG }\end{array}$ \\
\hline $\mathrm{MBP}$ F $4,5 \rightarrow \mathrm{S}$ & $\begin{array}{l}\text { for.: GATGAAAACCCAGTAGTC } \\
\text { CATTCATCCAAGAACATTGTGACACC } \\
\text { rev.: GGTGTCACAATGTTCTT } \\
\text { GGATGAATGGACTACTGGGTTTTCATC }\end{array}$ \\
\hline $\mathrm{MBP}$ F6 $\rightarrow \mathrm{S}$ & $\begin{array}{l}\text { for.: GCCTGTCCCTCAGCAGATCTA- } \\
\text { GCTGGGGAGGAAGAGACAG } \\
\text { rev.: GCCTGTCCCTCAGCAGA } \\
\text { TCTAGCTGGGGAGGAAGAGACAG }\end{array}$ \\
\hline Subcloning in pSF expression vector & $\begin{array}{l}\text { for.: TATTTTCAAGGATCCATGGCAT- } \\
\text { CACAGAAGAGACCC } \\
\text { rev.: CAGCTAATTAAGCTTAACA } \\
\text { ACTAGTGCGTCTCGCCATGGGAG }\end{array}$ \\
\hline
\end{tabular}

\subsubsection{Software}

The software used in this thesis are listed together with their purpose of usage in Table 2.5. 
Table 2.5: Software used in this study

\begin{tabular}{|c|c|c|}
\hline Software & Application & Source/Manufacturer \\
\hline Adobe Illustrator CS3 & Image processing & Adobe Systems, Inc. \\
\hline $\mathrm{ApE}$ & DNA analysis and editing & $\begin{array}{c}\text { Wayne Davis, University of } \\
\text { Utah }\end{array}$ \\
\hline GraphPad Prism & $\begin{array}{l}\text { Statistical analysis and } \\
\text { graph production }\end{array}$ & http://www.graphpad.com/ \\
\hline ImageJ & $\begin{array}{l}\text { Image processing and } \\
\text { analysis }\end{array}$ & http://rsbweb.nih.gov/ij/ \\
\hline ImageSP & $\begin{array}{c}\text { electron micrograph } \\
\text { acquisition }\end{array}$ & TRS, SysProg \\
\hline Leica Confocal Software & Confocal images acquisition & $\begin{array}{l}\text { Leica Microsystems, } \\
\text { Mannheim, Germany }\end{array}$ \\
\hline LaTeX and TeXworks & $\begin{array}{c}\text { Document writing and } \\
\text { editing }\end{array}$ & $\begin{array}{l}\text { http://www.latex- } \\
\text { project.org/ }\end{array}$ \\
\hline LSM software & Confocal images acquisition & Zeiss, Inc. \\
\hline Mendeley & Bibliography manager & http://www.mendeley.com/ \\
\hline
\end{tabular}

\subsection{Methods}

\subsubsection{Cell culture}

\subsubsection{Primary mouse oligodendrocytes}

Primary cultures of mouse oligodendrocytes were prepared from postnatal day zero (P0) mice in a modified protocol as described previously [323]. In brief, the mice were decapitated, the meninges and the hindbrain were removed, the brains were then trypsinized $(0.25 \%)$ for 10 mins at $37^{\circ} \mathrm{C}$. The brains were washed with Hanks buffered saline solution (HBSS) and subsequently, gently dissociated in Basal Medium Eagle (BME) supplemented with 10\% horse serum (HS) (PAA Laboratories GmbH, Pasching, Austria), $2 \mathrm{mM}$ GlutaMAX and $50 \mathrm{U} / \mathrm{mL}$ each of penicillin and streptomycin (Gibco, Invitrogen, Darmstadt, Germany). After passing the cell suspension through a $0.2 \mu \mathrm{m}$ cell sieve, the cells were plated in $75 \mathrm{~cm}^{2}$ flasks pre-coated with $100 \mathrm{mg} / \mathrm{mL}$ poly-L-Lysine (PLL; MW>300000) and then grown at $37^{\circ} \mathrm{C}$ with $7.5 \% \mathrm{CO}_{2}$ and $90 \%$ humidity for 7-10 days with intermittent medium changes. The oligodendroglial progenitor cells growing on top of a monolayer of astrocytes were obtained via differential shaking. After centrifugation of the media at $900 \mathrm{~g}$ for $10 \mathrm{mins}$, the cells were resuspended in SuperSATO media (for composition see Table 2.6). An appropriate number of cells (for low density: 15.000 cells $/ \mathrm{cm}^{2}$, for high density: 30.000 cells $/ \mathrm{cm}^{2}$ ) was then seeded onto PLL-coated dishes or coverslips (R.Langenbrinck, Labor- und Medizintechnik, Emmendingen, Germany). After platting, the cells were 
maintained in culture for 2 - 6 days before using for experiments.

Table 2.6: Composition of SuperSATO medium

\begin{tabular}{c|c} 
Component in final concentration & vendor \\
\hline DMEM with $4.5 \mathrm{~g} / \mathrm{L}$ glucose & Gibco \\
$1 \mathrm{X}$ B-27 supplement & Gibco \\
$2 \mathrm{mM}$ GlutaMAX & Gibco \\
$50 \mathrm{U} / \mathrm{mL}$ penicillin/streptomycin & Gibco \\
$1 \mathrm{mM}$ sodium pyruvate & Gibco \\
$1 \%$ horse serum & PAA Laboratories \\
$50 \mathrm{U} / \mathrm{mL}$ each of penicillin and streptomycin & Gibco \\
0.52 M L-thyroxine & Calbiochem \\
\end{tabular}

\subsubsection{PtK2 cells}

PtK2 cells were kindly provided by Dr. Christian Eggeling, Max Planck Institute for Biophysical Chemistry, Göttingen, Germany. Cells were cultured in DMEM with $4.5 \mathrm{~g} / \mathrm{L}$ glucose supplemented with $10 \%$ FCS, $2 \mathrm{mM}$ GlutaMAX, $1 \mathrm{mM}$ pyruvate and $50 \mathrm{U} / \mathrm{mL}$ of penicillin and streptomycin. The PtK2 cells were cultured in $75 \mathrm{~cm}^{2}$ flasks at $37^{\circ} \mathrm{C}$ with $5 \% \mathrm{CO}_{2}$ and $90 \%$ humidity. When cells reached a confluency of $80-90 \%$, they were detached using $0.05 \%$ trypsin-EDTA and split 1:5 or 1:10. The detachment from the surface was stopped by adding fresh media with serum. For transfections, the cells were counted and an appropriate number was seeded onto pre-sterilized coverslips.

\subsubsection{Antibody Production with Hybridoma Cell Lines}

To generate vast amounts of antibodies immunoreactive againt Galactosylceramide (namely $\mathrm{O} 1$ epitope), sulfatides (subsequently O4 epitope) or myelin oligodendrocyte glycoprotein (MOG), the antibody producing hybridoma cell lines were obtained from Dr. Eva-Maria Krämer- Albers, Institute of Cellular Neurosciences, University of Mainz, Germany. The cells were grown in suspension and expanded in Roswell Park Memorial Institute medium (RPMI) media supplemented with 10\% FCS, 2 mM GlutaMAX and $50 \mathrm{U} / \mathrm{mL}$ of penicillin / streptomycin. After obtaining a sufficient amount of $75 \mathrm{~cm}^{2}$ flasks, the cells were further passaged by splitting them 1:1 diluting the conditioned media with fresh media without serum. When the serum 
content of the media was $1.25 \%$, the hybridoma cells started to produce the antibody. Upon confluency of the cells, their media was harvested and cleared by centrifugation. After aliquoting of the supernatant, the media was snap-frozen and stored at $-20^{\circ} \mathrm{C}$. In order to purify the antibody, the immunoglobulins were precipitated over night with $35 \%$ of saturated ammonium sulfate solution and then pelleted by centrifugation and dissolved in PBS of $10 \%$ of the original volume [324]. This antibody containing solution was further washed twice with PBS and then concentrated with Amicon Ultra15 Centrifugal Filter Units with 100 kDa membrane (Millipore, Darmstadt, Germany). The purified and concentrated antibody solution was aliquoted and stored at $-20^{\circ} \mathrm{C}$ for long-term storage.

\subsubsection{In vitro assays}

\subsubsection{Freezing and thawing of cell lines}

In order to store cell lines long-term in liquid nitrogen, the cells were grown until confluency in $75 \mathrm{~cm}^{2}$ flasks as described above. Cell suspensions were directly pelleted by centrifugation, whereas other cells lines were detached from the vessel before. The pellet of one $75 \mathrm{~cm}^{2}$ flask was resuspended in $1 \mathrm{~mL}$ of the appropriate basal media containing $50 \%$ FCS and $10 \%$ DMSO. The cells were then slowly frozen in Nalgene R Sterile Cryogenic Vials in a Nalgene R Cryo Freezing Container (both by Thermo Fisher Scientific, Roskilde, Denmark) over night at $-80^{\circ} \mathrm{C}$. Thereafter, the cells were stored long-term in the liquid nitrogen tank.

To thaw frozen cells, the cryovials were removed from the liquid nitrogen tank and transferred on dry ice. The vials were then thawed quickly in the water bath at $37^{\circ} \mathrm{C}$ and the content transferred in $30 \mathrm{~mL}$ fresh media in a $75 \mathrm{~cm}^{2}$ flasks. After attachment of the cells, the monolayer was washed with PBS and fresh media was added. The cells were further grown and passaged normally according to the cell type, as described in previous sections.

\subsubsection{Lipofection-based transient transfection}

Transient transfection of primary mouse oligodendrocytes with lipid vesicles containing DNA was carried out using Lipofectamine 2000 (Invitrogen, Carlsbad, CA, USA). Therefore, a high density of cells (120 000 cells) were used per well of a 12 well plates. In brief, $1.6 \mu \mathrm{g}$ plasmid DNA was diluted in $100 \mu \mathrm{L}$ Opti-MEM media and $3 \mu \mathrm{L}$ Lipofectamine 2000 reagent in another $100 \mu \mathrm{L}$ Opti-MEM media. The solutions were incubated for 5 mins at RT and then the Lipofectamine and DNA solutions were mixed. After another 
incubation at RT for 20 mins, the Lipofectamine DNA mix was added dropwise to the cells. After $16 \mathrm{~h}$ of transfection, the cells were fixed using $4 \%$ PFA with $0.25 \%$ glutaraldehyde.

\subsubsection{Treatment of primary mouse oligodendrocytes}

Primary mouse oligodendrocytes were treated with one of the following agents outlined in Table 2.7 at the indicated concentrations and times previously determined not to affect oligodendroglia viability as determined by MTT assay. After the treatment, the cells were usually fixed with 4\% PFA with $0.25 \%$ glutaraldehyde or lysed for Western blot analysis. 


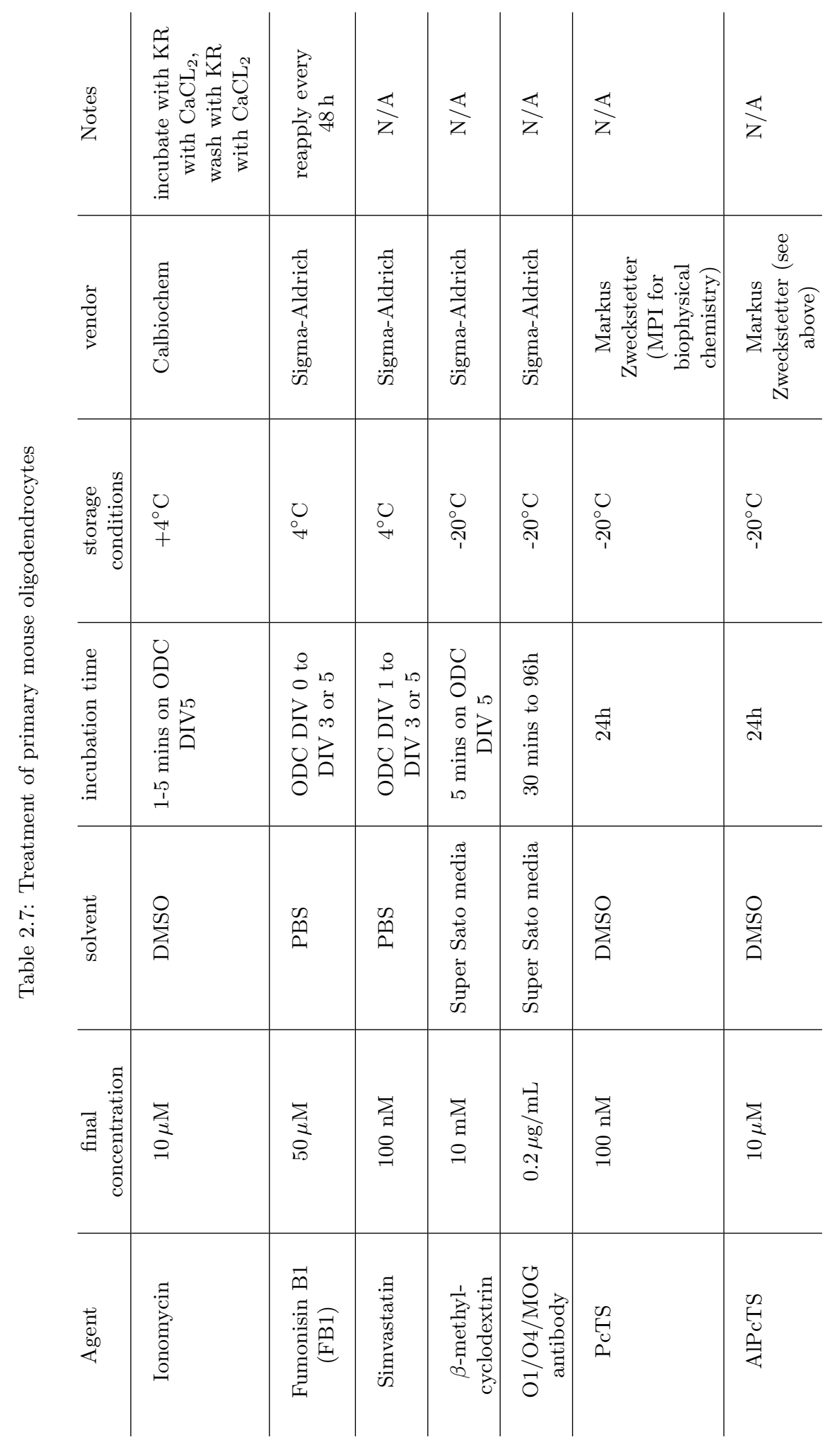




\subsubsection{Immunocytochemistry}

After fixation of cells with 4\%PFA and $0.25 \%$ glutaraldehyde, the monolayer was washed thrice with PBS and the cells were permeabilzed with $0.1 \%$ Triton X-100 for 2 mins. Thereafter, the cells were washed thrice with PBS and subsequently, blocked with $100 \%$ blocking solution for $1 \mathrm{~h}$ at RT. The cells were incubated with the desired primary antibodies in appropriate dilution in $10 \%$ blocking solution for $1 \mathrm{~h}$ at RT, washed three times for 5 mins with PBS, incubated with the secondary antibodies in required dilution in $10 \%$ blocking solution. After washing the monolayer extensively, the cells were mounted in Mowiol mounting medium. The list of antibodies used can be found in Table 2.1.

\subsubsection{Fluorescence resonance energy transfer}

To determine the self-interaction abilities of MBP after treatment with different chemicals or substances, fluorescence resonance energy transfer (FRET) was employed. Therefore, an antibody-mediated FRET approach was performed by coupling different fluorophores to the mouse monoclonal $\alpha$-MBP antibody (Covance, Lutherville, USA). Two set of antibody solution were prepared in $500 \mu \mathrm{L} 10 \%$ blocking solution and incubated on the roller for $1 \mathrm{~h}$ at RT: $1 \mu \mathrm{L}$ mouse $\alpha$-MBP antibody were incubated with 1:250 dilution of $\alpha$-mouse $(\mathrm{Fab})_{2}$ - Cyanine 3 (Cy3) or either with 1:250 dilution of $\alpha$-mouse $(\mathrm{Fab})_{2}$ - Cyanine 5 (Cy5). After the pre-incubation with the Fab-fragments, the mouse monoclonal antibody was fluorescently labeled. Hundred $\mu \mathrm{L}$ of the Cy3-MBP antibody solution was mixed with $300 \mu \mathrm{L}$ of Cy5-MBP antibody solution. This solution could be used for staining for $1 \mathrm{~h}$ at RT after permeabilization and blocking of the fixed cells as described previously.

The FRET measurements were carried out on the Leica sp2 using the FRET acceptor bleach wizard and the settings displayed in Table 2.8. The acceptor $(\mathrm{Cy} 5)$ was bleached about $70 \%$ using 20 frames and regions of interest of different sizes were drawn in the MBP positive areas of the cells (avoiding processes devoid of MBP). After bleaching, the fluorescent intensity of the donor was measured and the FRET efficiency was calculated according to the following formula:

$$
F R E T_{\text {eff }}=\frac{D_{P O S T}-D_{P R E}}{D_{P O S T}}, \text { for all } \mathrm{D}_{\mathrm{POST}}>\mathrm{D}_{\mathrm{PRE}}
$$

The FRET efficiencies were measured for control cells, as well as for treated cells and compared using one-way ANOVA (see sect. Statistics for details) 
Table 2.8: Settings on confocal microscope to measure FRET efficiency

\begin{tabular}{c|c|c|c} 
Fluorophore & $\begin{array}{c}\text { laser wavelenght in } \\
\mathrm{nm}\end{array}$ & laser power in $\%$ & $\begin{array}{c}\text { photomultiplier tube } \\
\text { settings in nm }\end{array}$ \\
\hline Donor $(\mathrm{Cy} 3)$ & 561 & 25 & $571-625$ \\
\hline Acceptor $(\mathrm{Cy} 5)$ & 633 & 30 & $645-700$
\end{tabular}

\subsubsection{Perfusion and Immunohistochemistry}

\subsubsection{Perfusion and Preparation of tissues for immunohisto- chemistry}

After the mouse was anaesthetized with an appropriate dose of avertin injection ip, the reflexes of the mouse was tested on the feet and eyes. Intracardiac perfusion was carried out by inserting a needle into the left ventricle and immediately cutting the right atrium after starting the perfusion with icecold filtered PBS with a Heraeus SR70 peristaltic pump $(0.2-0.5 \mathrm{~mL} / \mathrm{sec})$. After the blood was washed out as indicated by the yellow colour of the liver, the perfusion with freshly prepared filtered $4 \%$ PFA was carried out till animal stiffened (about 10 mins). The brain was removed immediately from the skull, whereas the whole spine was removed and immersion-fixed over night in $4 \%$ PFA. After another overnight fixation in 1\% PFA, the spinal cord was carefully removed from the spine and all tissues were incubated in 30\% sucrose till they sunk. Thereafter, the tissues were frozen in Tissue-Tek Cryo-OCT (Thermo Scientific, Epsom, United Kingdom) and stored at $-80^{\circ} \mathrm{C}$. The tissues were cut using the Leica cryostat CM1850 and stored in cryoprotective solution ( $25 \%$ glycerol, $25 \%$ ethylene glycol in PBS) at $-20^{\circ} \mathrm{C}$.

\subsubsection{Immunohistochemistry of cryosections}

In order to stain cryosections, the appropriate sections were washed in PBS thrice for at least $15 \mathrm{mins}$ and then mounted on slides before drying over night at RT or processed free-floating. The sections were then rehydrated in PBS followed by three washes with PBS for 15 mins and if necessary, antigen retrieval was carried out by boiling the sections thrice for 3 mins in $10 \mathrm{mM}$ citrate buffer ( $\mathrm{pH} 6.0$ ). After allowing the sections to cool down, blocking was carried out in 5\% HS, $5 \%$ GS and $5 \%$ FCS in $0.5 \%$ Triton-X 100 (blocking solution) for $1 \mathrm{~h}$ at RT. In case of using an mouse antibody on mouse tissue, an additional blocking step was performed using a 1:100 dilution of unlabeled goat anti-mouse IgG and IgM (Dianova, Hamburg, 
Germany) in blocking solution for $1 \mathrm{~h}$ at RT . The sections were incubated with the desired primary antibodies in appropriate dilution in $3 \% \mathrm{HS}$ in $0.5 \%$ Triton X-100 over night. The next day, the sections were washed three times extensively in PBS before incubated with the secondary antibodies in required dilution and the nuclear counterstain DAPI in $0.1 \mu \mathrm{g} / \mathrm{mL}$ in $3 \% \mathrm{HS}$ in $0.5 \%$ Triton X-100 for $1 \mathrm{~h}$ at RT. After washing the sections thrice with PBS, they were mounted using mowiol and dried over night before imaging.

\subsubsection{Luxol fast blue-cresyl violet staining}

For chemical staining of myelin, in particular glycolipids and glycoproteins for light microscopy, the Klüver-Barrera protocol for Luxol fast blue staining (LFB) was performed [325]. In brief, cryosection mounted on slides were dehydrated by subsequent 5 min incubation in the following solutions: $\mathrm{H}_{2} \mathrm{O}$, $70 \%$ ethanol, $90 \%$ ethanol, $100 \%$ ethanol. After dehydration, the sections were incubated over night in $0.1 \%$ luxol fast blue (Chroma, Münster, Germany) in $96 \%$ ethanol supplemented with $8.7 \mathrm{mM}$ acetic acid. The next day, the sections were washed in $96 \%$ ethanol and $\mathrm{ddH}_{2} \mathrm{O}$, followed by differentiation in $0.1 \%$ lithium carbonate (in $\mathrm{H}_{2} \mathrm{O}$ ) and subsequently, $70 \%$ ethanol till the background appeared clear. The cytoplasm of neurons was stained with $0.2 \%$ cresylviolet $(\mathrm{pH} 4.5$ ) in $20 \mathrm{mM}$ acetate buffer for 20 mins. After extensive washing of the sections in water, the tissue was dehydrated again and mounted with Eukitt (Kindler, Freiburg, Germany).

\subsubsection{Molecular Biology}

\subsubsection{Site-directed mutagenesis}

In order to exchange only a few amino acids of a protein, site-directed mutagenesis was employed by amplifying the whole plasmid using one set of primers containing the mutation and annealing to opposite strands. The newly synthesized DNA is not methylated, whereas the parental DNA is and can be digested with DpnI. The plasmid DNA containing the mutation can then be transformed.

For the primer design, it is important that the primer is between $25-45$ base pairs (bp) and the mutation should be in the middle of the primer. The primers should anneal to the same sequence on opposite strands and have a $\mathrm{GC}$ content of $>40 \%$. Ideally, the primers terminate with a $\mathrm{C}$ or $\mathrm{G}$ and the melting temperature $\left(\mathrm{T}_{\mathrm{m}}\right)$ should be above $78^{\circ} \mathrm{C}$ according to the following formula: 


$$
\mathrm{T}_{\mathrm{m}}=81.5+0.41(\% \mathrm{GC})-675 / \mathrm{N}-\% \text { mismatch }
$$

with

$\mathrm{n}=$ primer length in $\mathrm{bp}$

$\mathrm{GC}=\mathrm{GC}$ content of the primer

mismatch $=$ mismatch of $\mathrm{bp}$

For the polymerase chain reaction (PCR), $125 \mathrm{ng}$ of each primer, 30-50 ng plasmid DNA, $0.5 \mathrm{mM}$ deoxynucleotide triphosphates (dNTPs) and $10 \%$ dimethyl sulfoxide (DMSO) are diluted in $1 \mathrm{X}$ reaction buffer and 2.5 PfU DNA polymerase (Thermo Scientific, Waltham, USA) were added in a total reaction volume of $50 \mu \mathrm{L}$. The cycling conditions for the PCR for SDM are outlined in Table 2.9 .

After the PCR reaction, the parental (methylated) DNA was digested with $10 \mathrm{U}$ DpnI (New England Biolabs, Frankfurt, Germany) for $2 \mathrm{~h}$ at $37^{\circ} \mathrm{C}$ and subsequently, $10 \mu \mathrm{L}$ were transformed via heat-shock in chemically competent E.coli Dh5 $\alpha$ cells (Invitrogen, Darmstadt, Germany) according to the manufacturer's protocol.

Table 2.9: Cycling conditions for site-directed mutagenesis

\begin{tabular}{c|c|c|c} 
Segment & Cycles & Temperature in ${ }^{\circ} \mathrm{C}$ & Time \\
\hline 1 & 1 & 95 & 30 secs \\
\hline \multirow{2}{*}{2} & \multirow{2}{*}{18} & 95 & 30 secs \\
& & 55 & 60 secs \\
& 1 & 68 mins \\
\hline \multirow{2}{*}{3} & \multirow{2}{*}{18} & 5 mins
\end{tabular}

\subsubsection{Molecular Cloning}

For molecular cloning, the fragment of interest was first amplified by PCR, digested with restriction enzymes, ligated with T4 DNA ligase (Thermo Scientific, Waltham, USA) and transformed into chemically competent E.coli Dh5 $\alpha$ cells (Invitrogen, Darmstadt, Germany). For the PCR reaction, $100 \mathrm{ng}$ plasmid DNA, $0.25 \mu \mathrm{M}$ of each primer were added to 1X Phusion master mix (Thermo Scientific, Waltham, USA) and cycled according to the conditions outlined in Table 2.10 .

After the PCR reaction, the DNA fragment and the destination vector were digested with $10 \mathrm{U}$ of restriction enzymes in the appropriate buffer at the 
Table 2.10: Cycling conditions for molecular cloning

\begin{tabular}{c|c|c|c} 
Segment & Cycles & Temperature in ${ }^{\circ} \mathrm{C}$ & Time \\
\hline 1 & 1 & 98 & 3 mins \\
\hline 2 & 35 & 98 & 30 secs \\
& & $\mathrm{T}_{\mathrm{m}}+3^{\circ} \mathrm{C}$ & 60 secs \\
& & 72 & 30 secs per $\mathrm{kb}$ \\
\hline 3 & 1 & 72 & 5 mins
\end{tabular}

optimal working temperature of the enzymes. The digest was then subjected to gel electrophoresis and the bands of interest were cut and cleaned up from the gel using NucleoSpin Gel and PCR Clean-up (Macherey-Nagel, Düren, Germany) according to the manufacturer's protocol. Subsequently, the ligation reaction using $1 \mu \mathrm{L}$ of vector and $3 \mu \mathrm{L}$ of insert in a $1 \mathrm{X}$ T4 reaction buffer with $400 \mathrm{U}$ T4 DNA ligase (Thermo Scientific, Waltham, USA) over night at $24^{\circ} \mathrm{C}$. The next day, $3 \mu \mathrm{L}$ of the ligation reaction was transformed into chemically competent E.coli Dh $5 \alpha$ cells according to the manufacturer's protocol and plated on LB plates with the resistance of the destination vector. To screen for positive clones, DNA was prepared from an over night small scale culture and digested with the appropriate restriction enzymes. After obtaining positive sequencing results, larger scale preparations of DNA were obtained and stored at $-20^{\circ} \mathrm{C}$.

\subsubsection{DNA sequencing}

DNA sequencing was carried out by the AGCT laboratory of the Max Planck Institute for Experimental Medicine, Göttingen, Germany according to the Sanger method using the Big Dye Terminator Kit and analyzed on the 3730XL DNA-Analyzer (both Applied Biosystems, Foster City, USA).

\subsubsection{Biochemistry}

\subsubsection{Expression and Purification of recombinant MBP}

To obtain recombinant MBP, the desired construct containing wild type or mutated MBP $14 \mathrm{kD}$ DNA was cloned into pSF1625 with an N-terminal His-tag and C-terminal Cysteine for purification. The construct was then expressed in E. coli strain BLR harboring plasmid pRil and grown in Terrific broth (TB) media supplemented $50 \mathrm{mg} / \mathrm{mL}$ kanamycin and $37 \mathrm{mg} / \mathrm{mL}$ chloramphenicol at $37^{\circ} \mathrm{C}$. When the OD600 reached 0.6, the expression 
was induced with $1 \mathrm{mM}$ Isopropyl -D-1-thiogalactopyranoside (IPTG) and further shaken for $6 \mathrm{~h}$. The protein was purified essentially as described previously for the Nsp1 FG/FxFG repeat domain [326]. In brief, before cell harvest, $1 \mathrm{mM}$ PMSF and $5 \mathrm{mM}$ EDTA were added directly to the culture. The cells were resuspended in $8.3 \mathrm{M}$ guanidinium-hydrochloride (Gua- $\mathrm{HCl}$ ) containing $2 \mathrm{mM}$ EDTA and $20 \mathrm{mM}$ DTT and lysed by freezing and thawing once. After pelleting, the lysates was supplemented with $100 \mathrm{mM}$ Tris- $\mathrm{HCl}$ pH 8.5 and $1 \mathrm{mM}$ imidazole and applied to a nickel-column. After washing with $7.5 \mathrm{mM}$ Gua- $\mathrm{HCl}, 100 \mathrm{mM}$ Tris/HCl (pH 8.5), $1 \mathrm{mM}$ EDTA, $1 \mathrm{mM}$ imidazole, the beads were washed with $8 \mathrm{M}$ urea, $20 \mathrm{mM}$ Tris- $\mathrm{HCl} \mathrm{pH} 8.0$, $1 \mathrm{mM}$ EDTA, $1 \mathrm{mM}$ imidazole. The protein was eluted in the same buffer supplemented with $500 \mathrm{mM}$ imidazole. The eluate was then diluted 1:3 in water and applied to a thiopyridine-activated, SH-reactive matrix. These beads were washed with $6 \mathrm{M}$ Gua-HCl, $20 \mathrm{mM}$ Tris/HCl (pH 8.0), $1 \mathrm{mM}$ EDTA, $1 \mathrm{mM}$ imidazole and the bound protein was then eluted with $6 \mathrm{M}$ Gua-HCl, $20 \mathrm{mM}$ Tris-HCl pH 7.5, $10 \mathrm{mM}$ DTT. The eluate was applied to a C18 reverse phase HPLC column, eluted with increasing concentrations of acetonitrile in $0.15 \%$ trifluoroacetic acid. After lyophilization, the protein was stored at $-80^{\circ} \mathrm{C}$. For further biochemical assays, $1 \mathrm{mM}$ and $50 \mu \mathrm{M}$ stock solutions of each protein were obtained by dissolving the proteins in $\mathrm{ddH}_{2} \mathrm{O}$ and stored at $-80^{\circ} \mathrm{C}$.

\subsubsection{Preparation of myelin fractions}

Myelin in a pure preparation was obtained by sequential ultracentrifugation using a sucrose gradient. Brains used for obtaining myelin fractions were sonicated in 1 M HEPES, $500 \mathrm{mM}$ EDTA, 0.32 M sucrose and 1:50 dilution of protease inhibitor for 5 mins at $40 \%$ power in a tip sonicator till homogenized completely. The lysates were then subjected to ultracentrifugation using $0.32 \mathrm{M}$ and $0.85 \mathrm{M}$ sucrose (in $1 \mathrm{M}$ HEPES and $500 \mathrm{mM}$ EDTA) as discontinous gradient at $73.000 \mathrm{~g}$ for $30 \mathrm{mins}$. The interphase between the 0.32 and $0.85 \mathrm{M}$ sucrose phase was removed and washed thrice with water, first once at $73.000 \mathrm{~g}$ for $15 \mathrm{mins}$ and then, at $13.000 \mathrm{rpm}$. The pellet was then dissolved in water and subjected to another ultracentrifugation using 0.32 and $0.85 \mathrm{M}$ sucrose as gradient. After washing the pellet in the same way as after the first gradient centrifugation, the pellet was resuspended in $1 \mathrm{M}$ HEPES and $500 \mathrm{mM}$ EDTA, aliquoted and stored at $-80^{\circ} \mathrm{C}$.

\subsubsection{Preparation of Cells or Tissues for Biochemical Analysis}

In order to lyse cells for biochemical analysis, the cells were scrapped in an approriate volume of $1 \mathrm{X}$ lysis buffer supplemented with 1:50 dilution of the 
protease inhibitor stock solution on ice and incubated for 15 mins on ice. Then, the lysate was cleared by centrifugation at $27.000 \mathrm{rcf}$ for $10 \mathrm{mins}$ at $4^{\circ} \mathrm{C}$. The supernatant was collected and the protein content was determined using the Pierce BCA protein assay kit according to the manufacturer's protocol. For gel electrophoresis, the samples were boiled after addition of an approriate volume of $4 \mathrm{X}$ sample buffer at $60^{\circ} \mathrm{C}$ for $10 \mathrm{mins}$.

For lysis of tissues, the tissue was immersed in $1 \mathrm{X}$ lysis buffer supplemented with 1:50 dilution of the protease inhibitor stock solution and homogenised using an IKA UltraTurrax. After centrifugation at $10.000 \mathrm{rpm}$ for $10 \mathrm{mins}$ at $4^{\circ} \mathrm{C}$, the protein content of the supernatant was determined by using the Pierce BCA protein assay kit according to the manufacturer's protocol. The appropriate amount of protein was diluted with sample buffer and boiled before biochemical analysis.

\subsubsection{Detergent solubility assay}

To determine the detergent solubility of MBP after treatment with different compounds, the cells were scrapped and lysed for 30 mins on ice in $50 \mathrm{mM}$ Tris-HCl (pH 7.4), 5 mM EDTA, 20 mM 3-[(3-Cholamidopropyl)dimethylammonio]-1-propanesulfonate (CHAPS), 1:50 dilution of the protease inhibitor stock solution. After centrifugation at $17.000 \mathrm{rcf}$ for $30 \mathrm{mins}$ at $4^{\circ} \mathrm{C}$, the supernatant was taken off and the pellet was solubilized in an equal volume of the supernatant. After adding of the sample buffer, the lysates was boiled at $70^{\circ} \mathrm{C}$ for 10 mins and subsequently, western blotting was performed to assess a potential change in CHAPS solubility after treatment.

\subsubsection{Sodium Dodecyl Sulfate Polyacrylamide Gel Electrophoresis (SDS-PAGE)}

For separation of proteins according to their electrophoretic mobility, Sodium Dodecyl Sulfate Polyacrylamide Gel Electrophoresis (SDS-PAGE) was performed. The PAGE gels comprised of a $12.5 \%$ stacking and a resolving gel were self-casted with the composition outlined in Table 2.11. The samples with equal protein amount were loaded on the gel after boiling in sample buffer. On each gel, one lane was loaded with $5 \mu \mathrm{L}$ of the PageRuler Plus pre-stained protein ladder from Fermentas (St. Leon-Roth, Germany) to determine the size of the band of interest. Electrophoresis was performed at $100 \mathrm{~V}$ in the running buffer $(190 \mathrm{mM}$ glycine, $25 \mathrm{mM}$ Tris and $0.1 \%$ SDS ) till the dye front ran out of the gel. Subsequently, the PAGE gels were either coomassie stained to assess all protein bands or the gels were prepared for Western blotting to detect a specific protein using antibodies. 


\subsubsection{Western blot}

After the SDS-PAGE, the transfer of the proteins was carried out in a semidry approach to a Protran nitrocellulose membrane (GE Healthcare, Munich, Germany) for $1 \mathrm{~h}$ at $100 \mathrm{~V}$ in the transfer buffer $(20 \mathrm{mM}$ Tris, $153 \mathrm{mM}$ glycine, $20 \%$ methanol). The membrane was blocked in $5 \%$ non-fat milk in $1 \mathrm{X}$ TBS with $0.1 \%$ Tween 20 (TBST) for $1 \mathrm{~h}$ at RT and subsequently, incubated with the appropriate primary antibody over night in TBST. The next day, the membrane was washed thrice for at least 15 mins with TBST before the incubation with the appropriate horseradish peroxidase-conjugated secondary antibody diluted (usually in a 1:2000 dilution) in TBST for $1 \mathrm{~h}$ at RT. After washing the membrane three times for 15 mins with TBST, the membrane was developed by incubation with equal parts of peroxide solution and luminol enhancer solution detection reagents (Thermo Scientific, Schwerte, Germany) and subsequent, light sensitive CL-XPosure films (Thermo Scientific, Schwerte, Germany) were exposed to the membranes and developed in a Kodak X-OMAT 1000 image processor. The blots were scanned and analyzed using the ImageJ software by quantifying the intensity of the bands.

Table 2.11: Composition of Tris-PAGE gels

\begin{tabular}{c|c||c|c}
$12.5 \%$ resolving gel & volume & stacking gel & volume \\
\hline ddH ${ }_{2} \mathrm{O}$ & $3.0 \mathrm{~mL}$ & ddH ${ }_{2} \mathrm{O}$ & $2.0 \mathrm{~mL}$ \\
Tris-HCl $(1.5 \mathrm{M}, \mathrm{pH} 8.8)$ & $3.0 \mathrm{~mL}$ & Tris- $\mathrm{HCl}(0.5 \mathrm{M}, \mathrm{pH} 6.8)$ & $0.8 \mathrm{~mL}$ \\
$30 \%$ Acrylamide solution & $5.0 \mathrm{~mL}$ & $30 \%$ Acrylamide solution & $0.5 \mathrm{~mL}$ \\
$10 \%$ APS & $60 \mu \mathrm{L}$ & $10 \%$ APS & $30 \mu \mathrm{L}$ \\
TEMED & $30 \mu \mathrm{L}$ & TEMED & $15 \mu \mathrm{L}$
\end{tabular}

\subsubsection{Biomimetic assays}

\subsubsection{Liquid droplet assay}

Recombinant MBP was dissolved in deionized water at a concentration of $10 \mathrm{mg} / \mathrm{mL}$ and an equal volume of $2 \mathrm{mM} \mathrm{NaOH}$ was added. After thorough mixing, the opaque solution could be observed at $63 \mathrm{X}$ magnification and the about $10 \mu \mathrm{m}$ sized droplets were imaged in phase contrast microscopy.

\subsubsection{Preparation of lipid mixtures}

For all lipid vesicles, the desired lipid mixture and the appropriated volume to yield the desired mole percentage (composition and final concentration as 
indicated in Table 2.12) were added to chloroform in a pre-cleaned glass vial (Sigma-Aldrich, Munich, Germany). The lipid mixtures were dried at RT in the Vaccum Concentrator Speed Vac SC110 by Savant and then rehydrated and dissolved in the appropriated solvent (PBS or HBSS) by vortexing. In order to generate multilamellar vesicles, the opaque lipid solution was sonicated in a bath sonicator at $70^{\circ} \mathrm{C}$ until it became clear again.

Table 2.12: Lipids used for biomimetic studies

\begin{tabular}{|c|c|c|c|}
\hline lipid & vendor & Catalog number & molecular weight \\
\hline Cholesterol (Chol) & Sigma & C3045 & 386 \\
\hline $\begin{array}{l}\text { L- } \alpha \text {-lysophosphatidyl- } \\
\text { serine (PS) (brain) }\end{array}$ & Avanti & $840032 \mathrm{C}$ & 812 \\
\hline $\begin{array}{c}\text { L- } \alpha \text {-phosphatidyl- } \\
\text { ethanolamine (PE) (brain) }\end{array}$ & Avanti & $840022 \mathrm{C}$ & 746 \\
\hline $\begin{array}{l}\text { L- } \alpha \text {-phosphatidyl- } \\
\text { choline (PC) (brain) }\end{array}$ & Avanti & $840053 \mathrm{C}$ & 771 \\
\hline $\begin{array}{l}\text { L- } \alpha \text {-phosphatidylinositol } \\
\text { 4,5-bisphosphate (PIP2) (brain) }\end{array}$ & Avanti & $840046 \mathrm{X}$ & 1098 \\
\hline Sphingomyelin (SM) (brain) & Avanti & $860062 X$ & 731 \\
\hline
\end{tabular}

Table 2.13: Liposome compositions used in this thesis

\begin{tabular}{c|c|c}
$\begin{array}{c}\text { liposome } \\
\text { composition }\end{array}$ & lipid & $\begin{array}{c}\text { molecular part } \\
\text { in \% }\end{array}$ \\
\hline inner leaflet composition & Chol & 43,75 \\
PE & 27 \\
PIP2 & 2 \\
inner leaflet composition without Cholesterol & PC & 11.46 \\
& PS & 12.5 \\
& PIP2 & 3.13 \\
& PC & 27 \\
& PS & 12.5 \\
& SM & 3.13 \\
\hline inner leaflet composition without PS and PIP2 & Chol & 43,75 \\
& PE & 27 \\
& PC & 26.12 \\
& SM & 3.13
\end{tabular}


Table 2.14: GUV compositions used in this thesis

\begin{tabular}{c|c|c} 
GUV composition & lipid & molecular part in \% \\
\hline \multirow{2}{*}{ PC/PS } & PC & 66.6 \\
& PS & 33.3 \\
\hline \multirow{2}{*}{ PC/PS/SM } & PC & 50 \\
& PS & 12.5 \\
& SM & 37.5 \\
\hline \multirow{2}{*}{ PC/PS/SM/Chol } & PC & 25.1 \\
& PS & 18.75 \\
& SM & 18.75 \\
& Chol & 37.5
\end{tabular}

\subsubsection{Preparation of small unilamellar vesicles and supported lipid bilayer}

To generate small unilamellar vesicles (SUV's), the sonicated lipid mixture from 2.2.6.2 was tip sonicated at $4^{\circ} \mathrm{C}$ for 10 mins at $40 \%$ power. For spreading of the SUV's into supported lipid bilayers (SLB's), $250 \mu \mathrm{L}$ of the lipid mixtures was spread on pre-cleaned coverslips (see 2.2.6.4) for $1 \mathrm{~h}$ at RT and 15 mins at $37^{\circ} \mathrm{C}$.

\subsubsection{Preparation of glass slides for spreading of SUV's}

In order to obtain a uniform lipid monolayer, $18 \mathrm{~mm}$ glass coverslips were pre-cleaned with $2 \%$ of the detergent Hellmanex (Hellma Analytic, Müllheim, Germany) in bath sonicator at $70^{\circ} \mathrm{C}(100 \%$ power $)$ for 30 mins. After extensive washing in $\mathrm{ddH}_{2} \mathrm{O}$, the cleaned coverslips were stored immersed in water at $4^{\circ} \mathrm{C}$.

\subsubsection{Generation of Giant Unilamellar Vesicles (GUV's)}

In order to generate giant unilamellar vesicles (GUV's) by electroformation [327], the appropriate volume of the lipid stock solutions intended to be used were added to chloroform and dried at RT in an Eppendorf Vacuum Concentrator. Afterwards, the lipids were dissolved in chloroform and if intended, DPPE-Rhodamine (Invitrogen, Munich, Germany) was added for visualization at the microscope. Twenty five $\mu \mathrm{L}$ of lipid solution were spread 
on the conducting sides of indium-tin oxide (ITO)-coated glass slides (precleaned with a Hamilton syringe) (PGO, Iserlohn, Germany). The slides were dried at medium heat in an Eppendorf Vacuum Concentrator over night. The next day, the glass slides were assembled into chambers in such a way that the conducting sides of the ITO-coated glass sides were facing each other. The chamber containing $100 \mathrm{mM}$ sucrose was sealed with Hematokrit sealing paste (Brand, Wertheim, Germany) and on either side a copper tape was attached to connect to the electrodes. At 12 hertz in sinus wave, the voltage was increased stepwise to $1.6 \mathrm{~V}$ and then, the GUV's were grown in the chamber at $60^{\circ} \mathrm{C}$ over a time course of $3 \mathrm{~h}$. The GUV solution was removed from the chamber after budding the GUV's by switching to square wave at 5 hertz and then stored at $4^{\circ} \mathrm{C}$ for max. $2-3$ days.

\subsubsection{SLB-MBP GUV assay}

With the help of this assay, the self-association of MBP is assessed. Therefore, SUVs are spread on pre-cleaned coverslips as explained above see 2.2.6.3. After extensively washing the coverslips to remove unbound lipids, recombinant $\mathrm{MBP}$ at $7 \mu \mathrm{M}$ concentration was incubated to attach to the SLB for $1 \mathrm{~h}$ at RT. The unbound protein was washed off and then, GUV's composed of PC:PS in 2:1 molar ratio were added directly under the microscope to enable fast imaging (for their preparation see 2.2.6.5). After the GUV's sank to the SLB, the rate of bursted GUV's was calculated in $\%$ by considering 15 non-overlapping frames and representative images were taken.

\subsubsection{Liposome binding assay}

Liposomes were prepared by extrusion with the help of the mini-extruder by Avanti Polar Lipids (Alabaster, USA). As previously described, the lipid mixture $(1 \mathrm{mg} / \mathrm{mL})$ was dried and sonicated to generated multilamellar vesicles. By performing five freeze-thaw cycles (liquid nitrogen $/ 60^{\circ} \mathrm{C}$ ), these were broken in order to be able to generate unilamellar vesicles with the extrusion apparatus. This solution was passed through a polycarbonate membrane of appropriate size $(1 \mu \mathrm{m}, 400 \mathrm{~nm}, 200 \mathrm{~nm}, 100 \mathrm{~nm}) 20$ times to obtain a homogenous size distribution. $95 \mu \mathrm{L}$ of this solution was incubated with $2.5 \mu \mathrm{M}$ MBP recombinant protein (wild type or mutant) at RT and $250 \mathrm{rpm}$ for $1 \mathrm{~h}$. The binding of MBP was assessed by performing ultracentrifugation at $100.000 \mathrm{~g}$ on a Beckman TL-100 with a TLA 120.1 rotor. The pellet was resuspended in the same volume as the supernatant and fractions were subjected to Western blotting. 


\subsubsection{Spectroscopy}

\subsubsection{Circular dichroism (CD) spectroscopy in the presence of lipid vesicles}

The circular dichroism (CD) spectroscopy was carried out in collaboration with Arne Raasakka and Prof. Petri Kursula at the department of Biomedicine at the University of Bergen (for detailed information of method see [328] [329] [330]).

Peptides of the amphipathic helices of $14 \mathrm{kDa} \mathrm{MBP}$ with double phenylalanine residues (MBPp1; sequence: QDENPVVHFFKNIV; MBPp2, sequence: GILDSIGRFFSGDRGAPKR), as well as peptides with $\mathrm{F} \rightarrow \mathrm{S}$ substitutions at these motifs (MBPp1m, sequence: QDENPVVHSSKNIV; MBPp2m, sequence: GILDSIGRSSSGDRGAPKR), were obtained from JPT Peptide technologies (Berlin, Germany) and dissolved in water. CD spectra of the different peptides both in water and with $0.1 \%$ dodecyl phosphatidyl choline (DPC) micelles were measured on a Chirascan Plus instrument using a path length of $0.5 \mathrm{~mm}$ at $+20^{\circ} \mathrm{C}$ at a concentration of $0.2 \mathrm{mg} / \mathrm{ml}$. Synchrotron radiation $(\mathrm{SRCD})$ and oriented $\mathrm{CD}(\mathrm{OCD})$ spectroscopy were carried out on the SRCD beamlines UV-CD12 at ANKA (Karlsruhe, Germany) and DISCO at Soleil (France). In brief, for SRCD, the peptides were mixed with 1:1 DMPC/DMPG vesicles at a peptide/lipid molar ratio of 1:20. Three SRCD spectra were recorded in a quartz cell with a path length of $100 \mu \mathrm{m}$ and then averaged before normalising to the corresponding buffer. For OCD in lipid bilayers, $200 \mu \mathrm{l}$ of peptide/lipid mixtures (molar ratio 1:50) were spotted and dried on a UV-transparent quartz plate (Suprasil QS, Hellma Optik GmbH, Jena, Germany). The sample was then rehydrated to $97 \%$ relative humidity via the gas phase in the OCD chamber containing a saturated $\mathrm{K}_{2} \mathrm{SO}_{4}$ solution for $15 \mathrm{~h}$ at $30^{\circ} \mathrm{C}$. OCD spectra were recorded in $45^{\circ}$ angles of the sample cell rotating around the beam axis. The rotational spectra were subsequently averaged and normalised to the background spectra of lipid bilayers. In addition to PC/phosphoglycerol (PG), SRCD and OCD experiments were carried out with different lipid compositions (PC/PS, different ratios of $\mathrm{PC} / \mathrm{PG}$ ) and additives (sphingomyelin, cholesterol) and at different peptide/lipid ratios.

\subsubsection{Animal studies}

\subsubsection{Animal handling}

All experiments were performed in accordance with the german animal welfare law and the regulations of the State of Lower Saxony for animal experiments. The animals were kept in $12 \mathrm{~h}$ light dark-cycles and bred in the 
animal facility of the Max Planck Institute for Experimental Medicine.

\subsubsection{Cuprizone treatment}

For cuprizone time course studies, eight week old, male wild type (WT) C57B6 mice were fed with $0.2 \%$ cuprizone (Sigma-Aldrich, Munich, Germany) in powered chow [268] [272]. The animals were weighted once per week and if the body weight dropped below $20 \%$ of the starting weight, the animal was euthanized. The animals were treated for maximum 6 weeks and every week, one set of animals was perfused for histological studies, whereas the other set was used for electron microscopy.

\subsubsection{Experimental autoimmune encephalomyelitis (EAE)}

For induction of experimental autoimmune encephalomyelitis (EAE), Biozzi ABH mice were obtained from Harlan UK Ltd. (Biceister, UK). After two weeks of acclimatization to the animal unit, the mice were used for the experiments with 8 weeks of age. In order induce a relapsing EAE, per immunised animal, $50 \mu \mathrm{g}$ purified recombinant MOG protein dissolved in $300 \mu \mathrm{L}$ of $1 \mathrm{mg} / \mathrm{mL}$ complete Freund's adjuvants was used (incomplete Freund's adjuvant supplemented with $60 \mu \mathrm{g}$ Mycobacteria tuberculosis H37RA (both by Difco, Detroit, USA)). The solution was mixed using bridging syringues till a homogenous emulsion was obtained. After incubation of the emulsion at $4^{\circ} \mathrm{C}$, the animals were anaesthetized using ketamine/xylazine and $150 \mu \mathrm{L}$ were injected in either flank side subcutaneously. Furthermore, the animals received an ip injection of $50 \mathrm{ng}$ pertussis toxin (PTX) (List Biological Laboratories, Campbell, USA) on the day of the immunization and the day after. The procedure of immunization was repeated a week later (day $7,(\mathrm{~d} 7)$ ) and in addition, the animals again received $200 \mathrm{ng}$ PTX on d7 and $\mathrm{d} 8$. The animals were scored daily from d11 onwards for the development of the paralytic clinical disease. Animals were scored $0=$ normal, 1 for a fully flaccid tail, 2 for a impaired rigthing reflex, 3 for a hindlimb paresis, 4 for a complete hindlimb paralysis and 5 , if moribund or death. The mice were perfused in the peak of the acute (d17 to 18), in the first relapse (d25 to 30$)$ and chronic phase (d98) of EAE.

\subsubsection{Stereotactic injection of Lewis rats with antibodies}

Stereotactic injection of anti-MOG or anti-AQP4 antibody was carried out in adult Lewis rats obtained from Harlan (Horst, Netherlands). The injections were carried out by Anne Winkler at the Department for Neuropathology 
of the University of Göttingen in the group of Prof. Christine StadelmannNessler. The rats were anaesthetized by isoflurane inhalation anaesthesia and mounted in a stereotactic device. Access to the brain was obtained by drilling a fine hole through the skull $1 \mathrm{~mm}$ caudal to the bregma and $2 \mathrm{~mm}$ lateral to the sagittal suture. By inserting a glass capillary $3 \mathrm{~mm}$ deep, the corpus callosum (CC) was targeted and the rats were then injected with $1 \mu \mathrm{L}$ of MOG or AQP4 antibody solution with human complement over a 5 min period. As control, $1 \mu \mathrm{L}$ PBS and complement was injected in the contralateral site of the brain. Monastral blue (Sigma-Aldrich, Munich, Germany) was added to the solution injected as a marker dye for better visibility. After injection, the glass capillary was left in place for 5 mins before it was carefully withdrawn and the injection site was sealed by suture. Post-surgical recovery was uneventful in all cases, with no overt clinical signs.

\subsubsection{Acute brain slices of lesion site of AQP4 antibody injected Lewis rats}

After injection of Lewis rats with AQP4 antibody and complement, rats were decapitated at indicated times. Coronal slices were cut using a Leica VT1200S Microtome (Leica) at $200 \mu \mathrm{m}$ thickness in ice-cold artificial CSF (aCSF, $\mathrm{pH}$ 7.4). Afterwards, the acute slices were allowed to recover in aCSF at $35^{\circ} \mathrm{C}$ for $1 \mathrm{~h}$. The slices were then treated ex vivo with $25 \mathrm{mM}$ EGTA in aCSF or aCSF alone for $2 \mathrm{~h}$ at RT. ACSF was continuously bubbled with carbogen $\left(95 \% \mathrm{O}_{2}\right.$ and $\left.5 \% \mathrm{CO}_{2}\right)$ gas. The acute slices were then fixed with $4 \%$ PFA and subsequently stained with guinea pig GFAP (Synaptic systems), mouse QD9 (abcam) and rabbit MBP (Dako) as described above.

\subsubsection{Electron microscopy}

\subsubsection{Negative staining of liposomes for EM}

For negative staining of liposomes, a drop of the solution was placed on parafilm and fixed with the same volume of $0.2 \%$ glutaraldehyde. The Formwarcoated 100 hexagonal mesh copper EM grids (Plano, Wetzlar, Germany) were incubated on top of the drop for 5 mins. After washing, the samples were contrasted with $2 \%$ uranylacetate (SPI-Chem, Trezzo Sull Adda, Italy) for 15 secs and dried before imaging. Another method to achieve negative staining was to contrast with $2 \%$ neutral uranyl acetate (containing $0.7 \mathrm{M}$ oxalate titrated to $\mathrm{pH} 7$ with ammonium hydroxide) for 5 mins and then embed the samples in $1.8 \%$ methylcellulose and $0.4 \%$ uranyl acetate [331]. 


\subsubsection{Electron microscopy of high pressure frozen samples}

Animals used for electron microscopy (EM) studies were terminated by cervical dislocation and the brain was removed quickly. The brain was then cut in an appropriate orientation (sagital or coronal) with the help of the Leica vibratome VT1200S in $200 \mu \mathrm{m}$ sections. From these sections, the region of interest was punched and high pressure frozen in 20\% Poly-(vinylpyrrolidinone) (Sigma-Aldrich, Munich, Germany) using the Leica HPM100 (Leica, Wetzlar, Germany). The tissue was then freeze substituted according to the protocol in Table 2.15 using $0.1 \%$ tannic acid (Sigma Aldrich, Munich, Germany) and 2\% osmium tetraoxide (Sciences Services, Munich, Germany) using the Leica AFS II in a semi-automated fashion according to [332]. The Epon embedded tissue was cut with the Leica Ultracut S ultramicrotome (Leica, Wetzlar, Germany) and a Diatome diamond knife (Diatome, Biel, Switzerland) first in $0.5 \mu \mathrm{m}$ semithin sections that were subsequently stained with methylene blue / Azure II blue for $1 \mathrm{~min}$ to visualize myelin (lipid-rich) area in the tissue. Ultrathin section of $50 \mathrm{~nm}$ thickness of the region of interest were cut thereafter and contrasted with $4 \%$ uranylacetate (SPI-Chem, West Chester, USA) for 15 mins. Electron micrographs were obtained at the electron microscopes Zeiss EM900 or Zeiss EM912AB (Zeiss, Jena, Germany) equipped with a wide-angle dual speed 2K-CCD-camera (TRS, Moorenweis, Germany) using the ImageSP software.

For the preparation of the spinal cord, it was dissected freshly followed by embedding in $10 \%$ gelantine and cutting with the vibratome. The sections obtained were assessed with a light microscope to detect lesions within the white matter macroscopically and those displaying signs of lesions were high-pressure frozen ( $>3$ sections per animal, 5 animals). After freeze substitution and embedding, the samples were cut in semithin section $(0.5 \mu \mathrm{m})$ and stained with toluidine blue to detect lesions due to the lack of staining within the white matter. The samples containing lesions were further cut in ultrathin sections and processed for imaging. The staining of the semithin sections was used to detect the lesion site under the electron microscope. The contralateral site was used a control for the preservation of the tissue and normal myelination. Once the lesion site was localized, electron micrographs of the myelin pathology at the borders of the lesions were taken.

Three to five animals were used for each analysis. On cross-sections, randomly selected areas within the lesions were imaged per animal $(>25 \mathrm{im}-$ ages per condition). For the image analysis, the pictures were opened with the ImageJ software and the axons / myelin sheaths counted using the cell counter plug-in. Thereby, the myelin sheath was scored in one of the fol- 
lowing categories: intact, splitt (more than half of the axon diameter), vesiculated or degenerated. It should be noted that only those myelin sheaths were counted that could be categorized without doubts, hence only those that were clearly in focus were considered. Furthermore, the degenerated axons were counted by considering only those containing microtubules and neurofilaments. In the end, the percentage of the fragmentation profile was calculated for each time point.

Table 2.15: Automated freeze substitution protocol

\begin{tabular}{c|c|c} 
Solution & Duration & Temperature \\
\hline $0.1 \%$ tannic acid in acetone & $24 \mathrm{~h}$ & $-90^{\circ} \mathrm{C}$ \\
ice-cold acetone & $4 \times 30 \mathrm{mins}$ & $-90^{\circ} \mathrm{C}$ \\
$\begin{array}{c}2 \% \text { osmium tetroxide, } \\
0.1 \% \text { uranylacetate in acetone }\end{array}$ & $7 \mathrm{~h}$ & $-90^{\circ} \mathrm{C}$ \\
$"$ & $14 \mathrm{~h}$ & warm up to $-20^{\circ} \mathrm{C}$ \\
$"$ & $16 \mathrm{~h}$ & in $5^{\circ} \mathrm{C} / \mathrm{h}$ \\
$"$ & $2.4 \mathrm{~h}$ & $-20^{\circ} \mathrm{C}$ \\
& & warm up to $4^{\circ} \mathrm{C}$ \\
cold acetone & $1 \mathrm{~h}$ & in $10^{\circ} \mathrm{C} / \mathrm{h}$ \\
cold acetone & $4 \times 30 \mathrm{mins}$ & $4^{\circ} \mathrm{C}$ \\
acetone: epon mix $(2: 1)$ & $2 \mathrm{~h}$ & $4^{\circ} \mathrm{C}$ \\
acetone: epon mix $(1: 1)$ & $2 \mathrm{~h}$ & $\mathrm{RT}$ \\
acetone: epon mix $(1: 2)$ & $2 \mathrm{~h}$ & $\mathrm{RT}$ \\
$90 \%$ epon in acetone & over night & $\mathrm{RT}$ \\
fresh pure epon & over night & $\mathrm{RT}$ \\
& & $\mathrm{RT}$ \\
& & $\mathrm{RT}$
\end{tabular}

The tissue was removed from the samples carrier and placed in a gelatine capsules for embedding that were filled with pure epon. The blocks were polymerized for $24 \mathrm{~h}$ at $60^{\circ} \mathrm{C}$.

\subsubsection{Cryo-Immuno Electron microscopy}

For immunolabeling of cryosection, the brain sections were immersion-fixed in $4 \%$ PFA, $0.25 \%$ glutaraldehyde (EM grade, Science Services, Munich, Germany) in PB (pH 7.3) as described in Möbius et al. (2010) [332] [333]. After 2 days of fixation, the brain sections were infiltrated in $2.3 \mathrm{M}$ sucrose over night. Thereafter, the tissue was mounted on aluminium pins and snapfrozen in liquid nitrogen. The ultrathin Tokuyashu sections were cut on a 
Table 2.16: Antibodies for Cryo-immunoelectron microscopy

\begin{tabular}{c|c|c|c} 
Antigen & Host species & Dilution & Manufacturer \\
\hline MBP & rabbit & $1: 300$ & Dako Cytomat., Carpinteria, USA \\
PLP (A431) & rabbit & $1: 250$ & self-made from hybridoma [335]
\end{tabular}

Leica UC6 cryo-ultramicrotome using a cryoimmuno diamond knife (Diatome, Biel, Switzerland). The immunolabeling was performed as described in Peters and Pierson (2008) using one of the antibodies from 2.16 [334]. The rabbit antibody was detected using protein A gold $(10 \mathrm{~nm}, \mathrm{CMC}$, Utrecht, Netherlands) at a dilution of 1:20 for 20 mins. The sections were imaged with a Leo 912 AB electron microscope equipped with a $2 \mathrm{k}$-CCD camera (Proscan, Scheuring, Germany).

\subsubsection{Image Processing and Statistical Analysis}

For all immunohistological studies, the settings for the exposure time and the gain were maintained for the different fluorophores within one experiment in order to determine the increase in signal intensity. All images obtained at the epifluorescence microscope Leica DMI6000 or Leica confocal sp2 / sp5 (Leica, Wetzlar, Germany) were analysed in the ImageJ software by determining the integrated density (area multiplied by the signal intensity). For each time point or treatment, the mean, the standard deviation and the standard error of the mean was calculated. Statistical analysis was carried out using the GraphPad Prism software: for the comparison of two groups with normal distribution, unpaired student's t-test was carried out, whereas for the analysis of multiple groups one-way ANOVA was used. P-values above $0.05(\mathrm{P}>0.05)$ were considered non-significant (n.s.), hence $\mathrm{P}$-values below 0.05 were considered significant and marked with $*(\mathrm{P}<0.05=*)$. Pvalues smaller than 0.01 were displayed with $* *$ and $\mathrm{P}$-values below 0.001 with ***. 



\section{Chapter 3}

\section{Results}

"The great tragedy of science the slaying of a beautiful hypothesis by an ugly fact."

Thomas Henry Hexley

\subsection{Myelin pathology in demyelinating diseases}

\subsubsection{Ultrastructural characterization of myelin pathology in early NMO lesions}

Myelin fragmentation is a common feature in MS and other demyelinating diseases. In order to understand the molecular events leading to myelin pathology and subsequent demyelination, we employed different animal models known to lead to demyelination in vivo. As outlined in 1.5.2, NMO patients have autoantibodies against AQP4 in their serum and these initiate an humoral response to astrocytic endfeet leading to their apoptosis, as well as secondary demyelination. In our experiments, we employed an human recombinant antibody against AQP4 that was derived from the CSF of NMO patient (rAB53, subsequently termed AQP4-antibody)[301] and injected this antibody stereotactically in the corpus callosum of Lewis rats. This focal NMO model is known to lead to ODC apoptosis and a robust demyelination after 7 days as determined by anti-MBP immunostaining [307] [309] [301]. In order to detect myelin fragmentation profiles, the AQP4 antibody (or PBS or complement as a negative control) was injected and we processed the lesions for EM to determine the myelin ultrastructure after different time points.

In order to visualize myelin in a state that is close to native, we processed 
the samples using high pressure freezing. This sample preparation avoids chemically fixation and dehydration [336] resulting in a better myelin preservation than conventional fixation for EM [332]. Therefore, the samples were obtained from unfixed tissue and then frozen under high pressure in liquid nitrogen atmosphere. This and subsequent freeze substitution prevents ice crystal formation, as well as dehydration of the tissue resulting in a better preservation of myelin.

To determine a time course of myelin degeneration, we prepared tissue samples from lesion sites after $1 \mathrm{~h}, 18 \mathrm{~h}, 5$ days and 7 days post injection (p.i.) of the antibody. Semithin sections stained with toluidine blue, as well as the needle track and for later time points immune cells infiltration were used to localize the lesion site in the samples. Careful examination of the lesion sites generated by PBS injection was employed to establish a measure for the needle damage induced by injection. For assessing the pathology by the AQP4 antibody, this zone around the lesion site was omitted and images were acquired further away from this region. The acquired electron micrographs were quantified by counting the fragmentation profiles and the total number of myelin sheaths on the images and then assessing the percentage of different fragmentation pattern that could be identified unequivocally (see Figure $3.1 \mathrm{C}$ ).

At one hour p.i., myelin was still intact and indistinguishable from the lesions obtained for PBS injections.Previous experiments could show that there is ot difference between PBS or complement injection (see Figure 5.2). After $18 \mathrm{~h}$ p.i., a large degree of the myelin sheaths around the lesion with the AQP4 antibody were showing myelin pathology and $70 \%$ of the myelin sheaths were vesiculated at the innermost layers (see Figure $3.1 \mathrm{~B}$ with mild vacuolization). At this time point, the astrocyte pathology was already apparent and microglia/macrophage infiltration was observed (data not shown). Five days p.i., the lesion size was increased (macroscopic oberservation) and myelin sheaths showed a more severe degree of vesiculation as less compacted layers were remaining around the large vacuoles of myelin membrane (see Figure $3.1 \mathrm{~B}$ with severe vesiculation). The percentage of myelin sheath with a milder vesiculation decreased, whereas a large proportion of sheaths were severely vesiculated or completely degenerated at this time point. Frequently, the degenerated myelin sheaths were not seen in association with the axons they previously ensheathed (see Figure 3.1 B with degenerated myelin sheath). After 7 days p.i., the lesion size further increased and at the border of the lesions, the degree of myelin pathology decreased as an higher percentage of normally myelinated sheaths were detected. The myelin fragmentation patterns detected at this time point were very heterogenous with variable degree of vesiculations, as well as degenerated myelin sheaths were observed. Furthermore, this time point displayed a high degree of axons ensheathed with two myelin sheaths suggesting that the inner myelin stack lacks the signalling molecules repulsive for further myelination. 
Assessing the electron micrographs of the time course of the focal NMO lesions also reveal that the acute axonal damage was apparent mostly at the stage of severe or degenerated myelin. Here, the axons lost their round/oval shape, presented with an usually dark contrast and were devoid of cytoskeletal proteins. The acute axonal damage also presented with swellings of the axon and interestingly, occured at a rather late stage of the time course (see Figure 5.3). Hence, mild vesiculation of the inner myelin sheath does not seem to affect the axonal integrity at this stage.

Taken together, these data suggest that the first signs of myelin pathology are vesiculation of the innermost layer of myelin and over time, the vesiculation seems to involve more layers of the previously compacted sheath. At late stages, the myelin stack is then completely degenerated before it is phagocyted by microglia or other immune cells. 
A
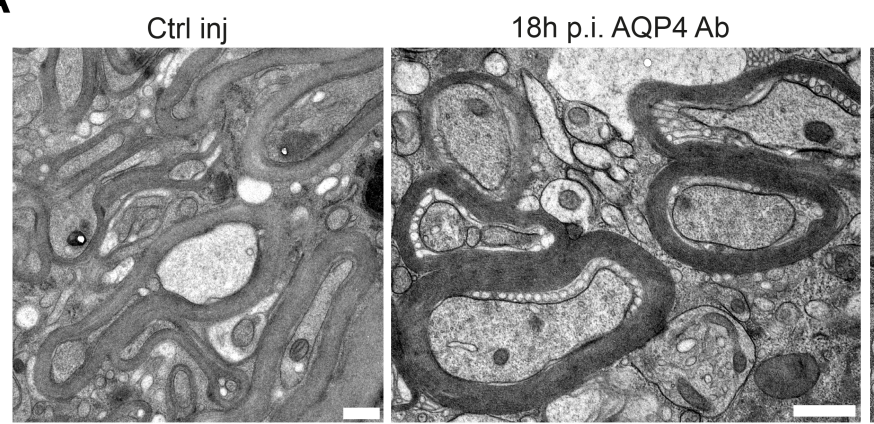

5d p.i. AQP4 Ab

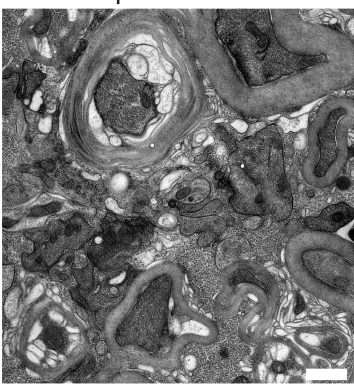

B

$$
\text { Intact }
$$

Mild

Severe Degenerated
myelin sheath

Double

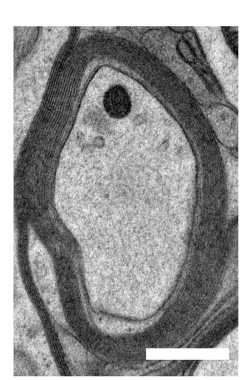

vesiculation
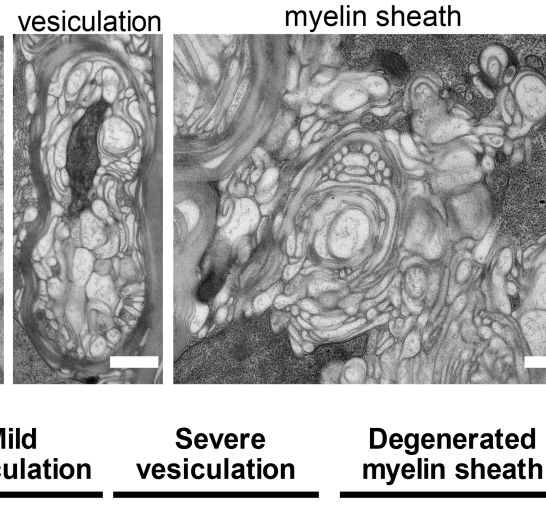
myelin sheath

C

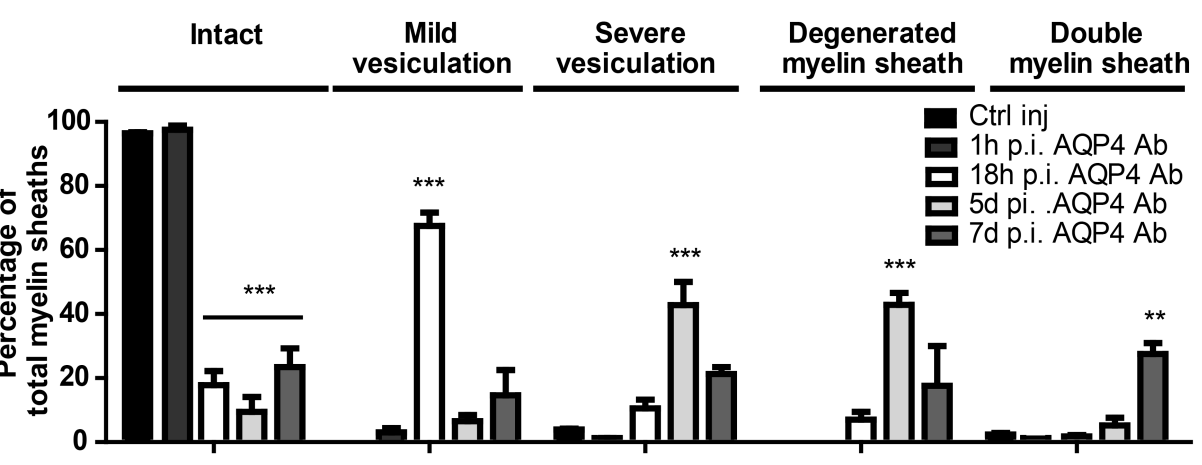

Figure 3.1: Myelin pathology in early NMO lesions

Lewis rats were injected with AQP4 antibody and tissues were processed after the indicated times for EM. The myelin fragmentation profiles observed after AQP4 injection are shown with representative images in B (scale bar $500 \mathrm{~nm}$ ), whereas $\mathrm{C}$ shows their quantification at different time p.i. ( $1 \mathrm{~h}, 18 \mathrm{~h}, 5 \mathrm{~d}$ and $7 \mathrm{~d}$ p.i.). Bars shown mean with SEM ( $\mathrm{n}=3$ animals, $>300$ axons per animal, ${ }^{* *}=\mathrm{P}<0.01,{ }^{* * *}=\mathrm{P}<0.001$, one-way ANOVA).

\subsubsection{Loss of MAG staining in focal NMO lesions}

Previous studies of early NMO lesions or a subset of MS patients could show a loss of MAG staining before other myelin proteins, such as MBP and PLP, were decreased [213] [294] [201] [295] [337]. As vesicular degeneration of innermost myelin sheaths in EM was the first sign of myelin pathology 
after injection of the AQP4 antibody and MAG is localised to the adaxonal space of myelin, we hypothesized that a decrease in MAG staining could also be identified in our focal NMO model. Therefore, we stained lesion sites of AQP4 antibody or PBS with complement after $1 \mathrm{~h}$ p.i., $18 \mathrm{~h}$ and $24 \mathrm{~h}$ p.i. with MBP and MAG (see Figure 3.2). The former time point did not display an altered MBP to MAG ratio, whereas after $18 \mathrm{~h}$ p.i. of the AQP4 antibody injection, the lesions displayed a significant reduction in MAG and p25 staining for mature oligodendrocytes (see Figure 3.2 C). At these time points, the MBP intensity was similar suggesting that the MBP to MAG ratio can be used as a histological marker for the fragmentation of the inner myelin layers in early NMO lesions. Despite that at $3 \mathrm{~d}$ p.i. also the MBP signal intensity was decreased suggesting a increased degree of myelin pathology.

Similar results were acquired in collaboration with Dr. Anne Winkler, Dr. Claudia Wrzos and Prof. Stadelmann-Nessler, University Medical Center Göttingen for human biopsies of NMO patients as the lesions could be staged depending on their immunohistochemical signal for MAG and MBP (see Figure 3.3). In early stage lesions, the selective MAG loss is associated with an active inflammation as indicated by MRP14 positive inflammatory cells. For late stage lesions, where MAG and MBP was lost from the lesion area the inflammatory cell number was decreased. These results suggested that MAG loss in early stage lesions can be correlated to an ongoing inflammation.

\subsubsection{Myelin pathology in Lewis rats injected with MOG antibody}

Apart from AQP4, antibodies against MOG are also implicated in a subset of NMO patients and infantile acute disseminated encephalomyelitis that are sero-negative for AQP4 antibodies [338] [339] [318]. AQP4 antibodies lead to the death of astrocytes and cause subsequent demyelination [307]. In contrast to that, MOG antibodies lead to a direct attack of the ODC by binding to MOG localized to the abaxonal space. We determined the myelin fragmentation patterns caused by the stereotactic injection of an humanised anti-MOG antibody [292] [340] or PBS with complement into the corpus callosum of Lewis rats and then carried out high pressure freezing. Due to the direct binding of the MOG antibody on myelin, shorter time points p.i. were chosen as compared to the AQP4 antibody injection. Furthermore, initial experiments detected nearly complete demyelination at $15 \mathrm{~h}$ p.i. of the antibody and therefore, even earlier time points were assessed.

As a control, $1 \mathrm{~h}$ p.i. of PBS or MOG antibody lesions were examined to establish the area of the needle damage. The lesion detection and the quantification of the myelin fragmentation pattern observed was essentially carried out as described above for the focal NMO model (see 3.1.1). The two condi- 
A

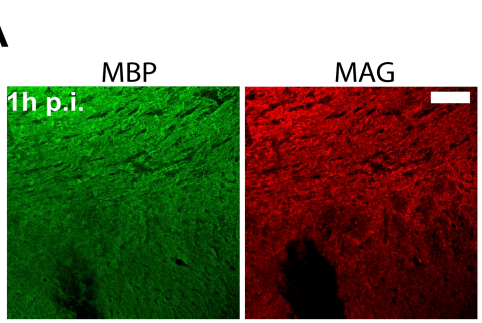

D

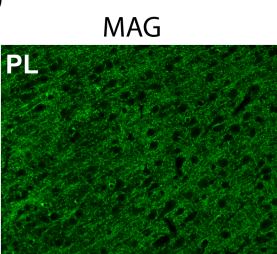

MBP

B
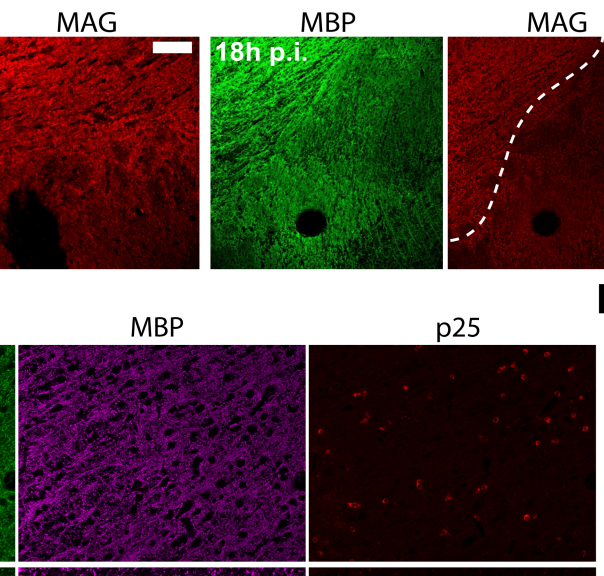

E

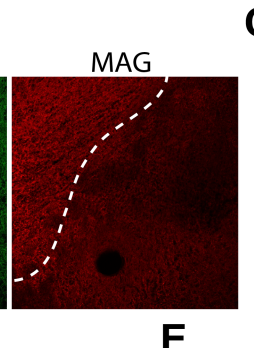

C
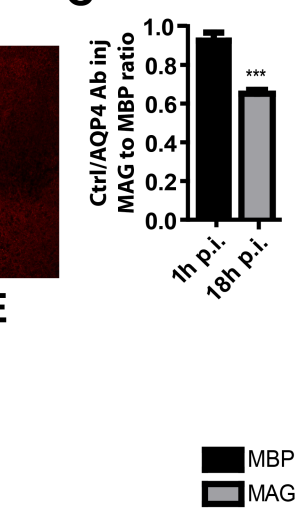
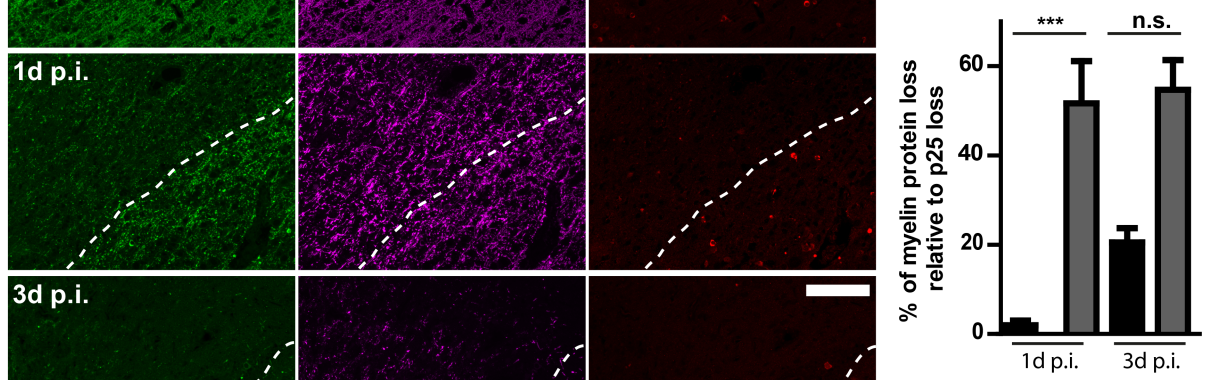

3d p.i.
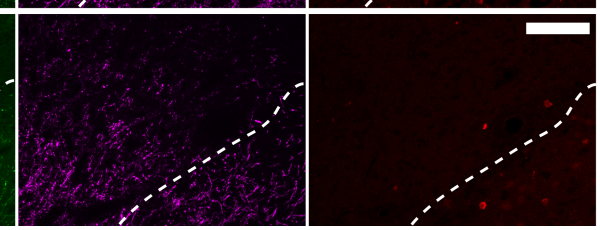

Figure 3.2: Early NMO lesions are devoid of MAG staining

Representative images of coronal sections of adult Lewis rat brains injected with human AQP4 antibody for $1 \mathrm{~h}(\mathrm{~A})$ or $18 \mathrm{~h}$ p.i. (B) stained with MBP in green and MAG in red (scale bar $1 \mathrm{~mm}$ for $\mathrm{A}$ and $\mathrm{B}$; dotted line indicates lesion border as identified by astrocyte loss). The quantification of the ratio of signal intensity of MBP and MAG is shown in C. Bars show mean with SEM ( $n=3$ animals, 3-5 regions of same size per animal, ${ }^{* *}=\mathrm{P}<0.001$, Student's t-test). (D) Time course of focal NMO lesions stained with MAG (in green), MBP (in purple) and p25 for mature oligodendrocytes (in red) (scale bar $200 \mu \mathrm{m}$, PL for perilesion, dotted line indicates lesion border as identified by astrocyte loss). The quantification of the area of the protein loss relative to the area of the p25 loss is displayed in percent. Bars show mean with SEM $\left(\mathrm{n}=3,{ }^{*}=\mathrm{P}<0.05,{ }^{* *}=\mathrm{P}<0.01\right.$, $* * *=\mathrm{P}<0.001$, one way-ANOVA). (D,E) Data acquired in collaboration with Dr. Anne Winkler and Prof. Stadelmann-Nessler at the University Medical Center Göttingen. 
A

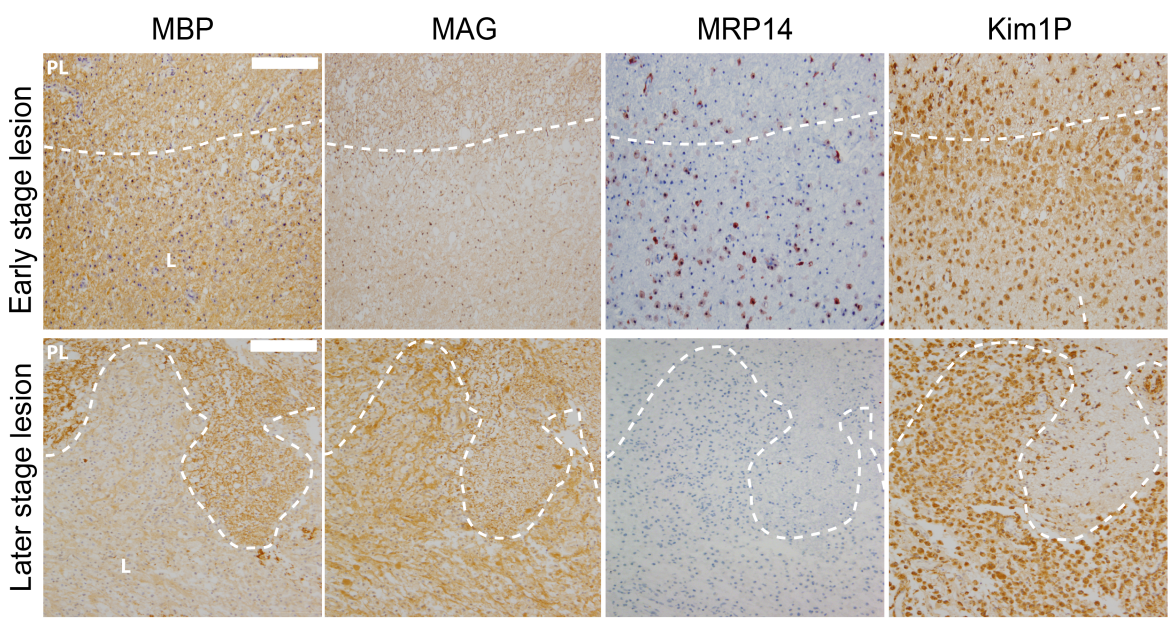

B

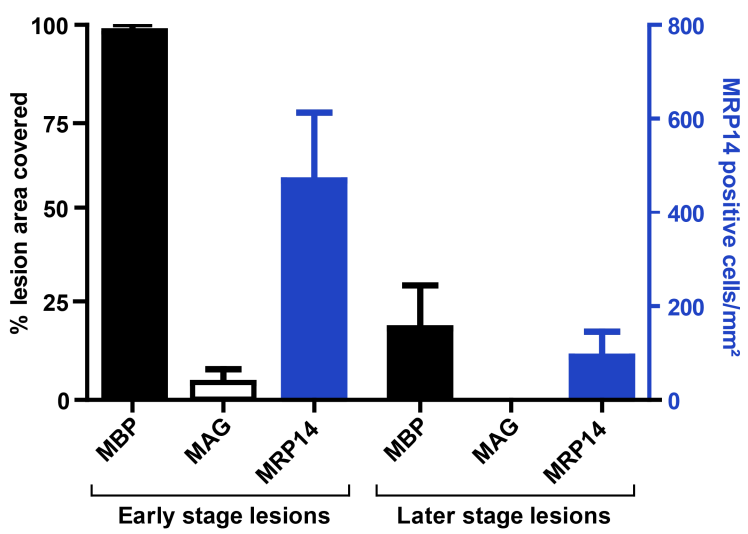

Figure 3.3: Early loss of MAG staining prior to MBP loss in human NMO lesions

(A) Representative images of immunohistochemistry of MBP, MAG, MRP14 and KiM1P of biopsies from NMO patients of the CNS $(\mathrm{n}=4)$ (scale bar $200 \mu \mathrm{m}$, PL for perilesion, $\mathrm{L}$ for lesion, dotted line indicates lesion border as identified by astrocyte loss). (B) The lesion area was identified based on GFAP and AQP4 loss and KiM1P positive macrophages. The quantification shows the lesion area covered by the MBP and MAG immunohistochemical signal and MRP14 positive early invading monocyted. Bars show mean with SEM ( $\mathrm{n}=3$ for early and late stage). Data acquired in collaboration with Dr. Claudia Wrzos and Prof. Stadelmann-Nessler at the University Medical Center Göttingen. 
tions did not display any differences at that time point suggesting that there is no myelin pathology after $1 \mathrm{~h}$ p.i.. After $8 \mathrm{~h}$ p.i. of the MOG antibody, first signs of myelin pathology (see Figure 3.4) could be detected in about $50 \%$ of the sheaths examined: in about $20 \%$ of myelin sheaths, splittings in the compact myelin were detected, whereas an increased proportion of $12 \%$ of ODC's had an enlarged inner tongue. Furthermore, vesiculation of the inner myelin sheaths could be detected in about $17 \%$ of myelin sheaths. At $12 \mathrm{~h}$ p.i., $18 \%$ of total myelin sheaths had an enlarged inner adaxonal space, whereas vesiculated inner myelin layers were increased to $25 \%$ of all myelin layers assessed. Hence, these fragmentation patterns increase in percentage compared to the earlier time point. Additionally, severely vacuolized myelin could be detected in about $12 \%$ of sheaths.

Hence, we concluded that stereotactic injection of the MOG antibody leads to split myelin sheaths and a swollen inner tongue, as well as vesiculated inner myelin layers. Over time, the myelin vesiculation seemed to increase in percentage and severity. At the early time points investigated, no significant axonal damage was apparent (see Figure 5.4). As the MOG antibody injection had a rather localized effect, no MAG immunohistochemistry was carried out. 
A
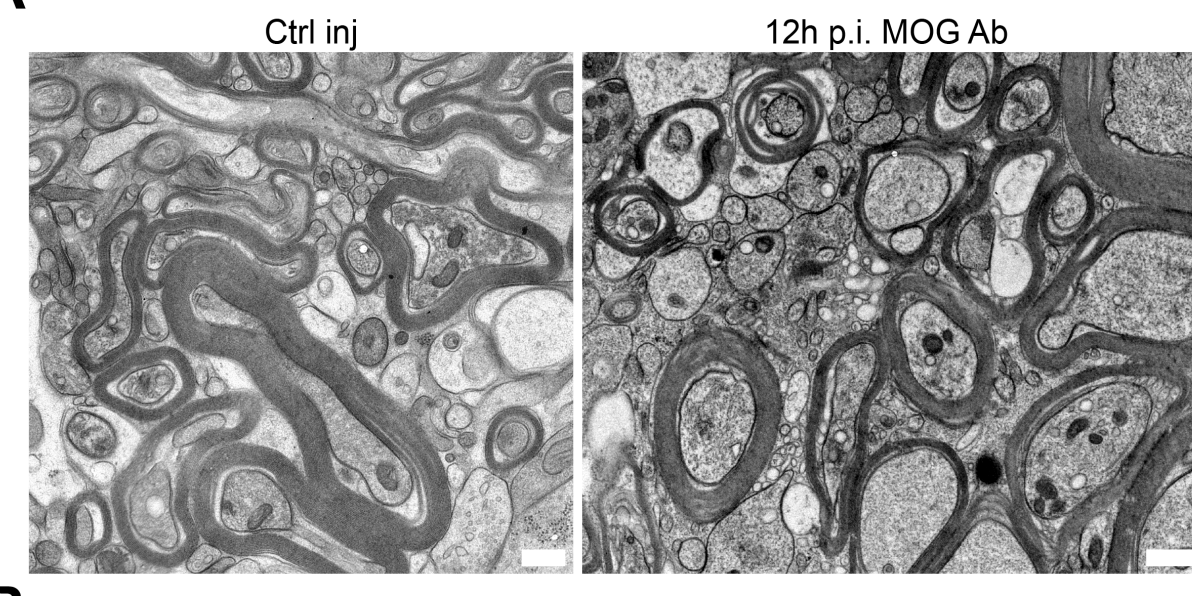

B

Intact

Split

Enlarged inner

Vesiculation

myelin sheath tongue

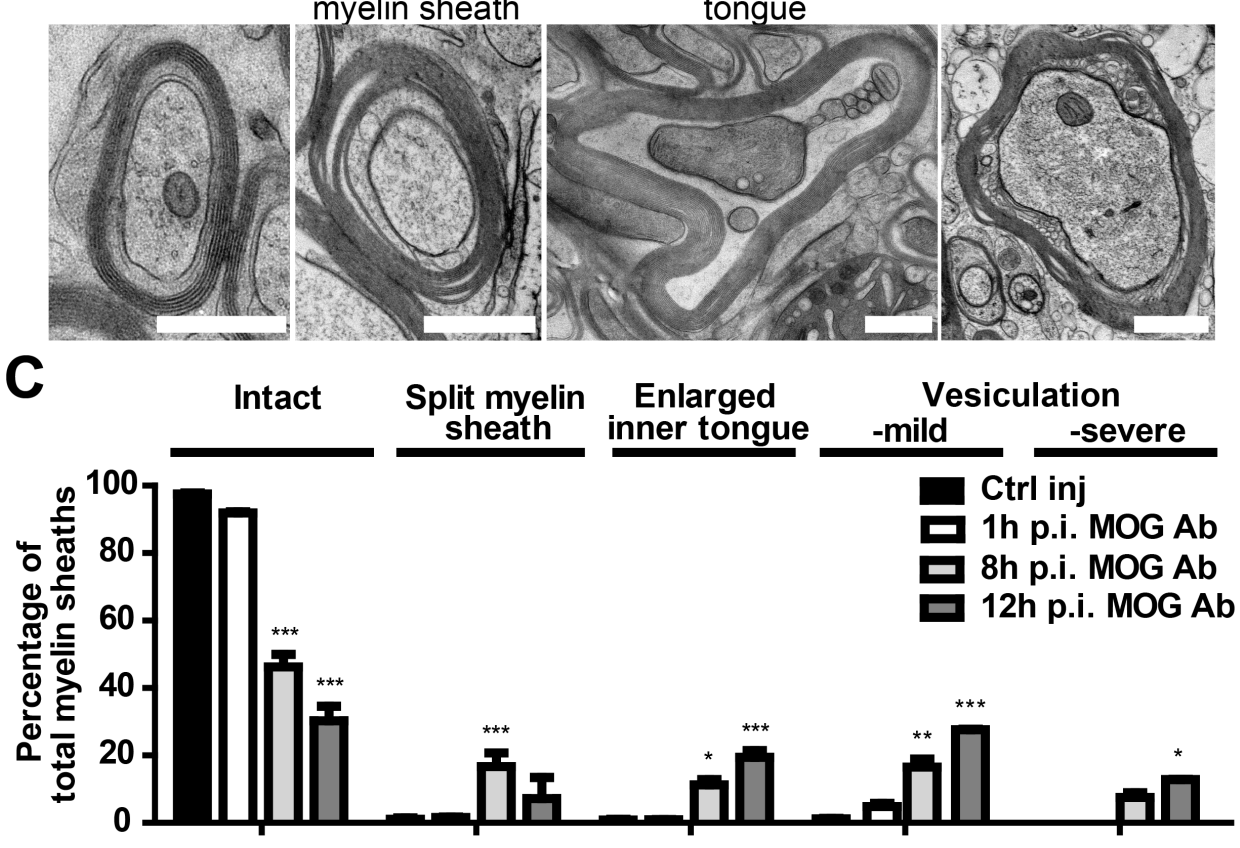

Figure 3.4: Early lesions of MOG antibody injection display enlarged inner tongue and vesiculation of inner myelin layers

In A, representative images of cross-sections of MOG antibody or PBS injection in corpus callosum of adult Lewis rats are shown at $12 \mathrm{~h}$ pi (scale bar $500 \mathrm{~nm}$ ). The upper panel in $\mathrm{B}$ depicts different myelin fragmentation profiles observed and the graph in $\mathrm{C}$ displays their quantification as percentage of total myelin sheath at $1 \mathrm{~h}, 8 \mathrm{~h}$ or $12 \mathrm{~h}$ pi of MOG antibody or PBS. Bars display mean with SEM ( $\mathrm{n}=3$ animals, $>300$ axons per animal, $* \mathrm{P}<0.05, * *=\mathrm{P}<0.01,{ }^{* * *}=\mathrm{P}<0.001$, one-way ANOVA). 


\subsubsection{Myelin degradation in Biozzi experimental autoimmune encephalomyelitis}

To investigate whether myelin fragmentation also starts at the inner tongue in other immune-mediated animals models, we employed the frequently used experimental autoimmune encephalomyelitis (EAE) model. Therefore, Biozzi mice were used as these mice have a high general antibody titer and EAE seems to be exacerbated by MOG antibodies [283] [341]. Furthermore, EAE in these mice follows a relapsing-remittent form followed by a secondary progressive disease course [342] (see 1.5.1.3.3).

For our studies, the Biozzi mice were immunized with MOG protein emulsified in complete Freund's adjuvants (at d 0 and $\mathrm{d} 7$ ) and the spinal cord of the immunized animals was processed for high pressure freezing during their first relapse (d 25 to 30) (for details on preparation see 2.2.9.2). Representative electron micrographs of the myelin pathology at the borders of the lesions are shown in Figure 3.5 A and B. In contrast to the control sites that displayed normally myelin sheaths, myelin at the border of the lesion frequently exhibits an enlarged adaxonal space, as well as vesiculation of the innermost myelin layer to about $30 \%$ for both fragmentation patterns (see Figure $3.5 \mathrm{C}$ ). These fragmentation profiles were also detected in the other demyelinating animal models. Despite that, we could detect myelin sheath that were split and contained vesicules within compact myelin. Furthermore, signs of focal bulging were and minor axonal damage (see Figure 5.5) observed.

Taken together, all investigated autoimmune-mediated animals models displayed common myelin breakdown patterns. These include an enlarged inner tongue and vesiculation of inner myelin sheath prior to demyelination. Over time, the percentage of vesiculation increased and the degree of myelin layers involved progressed. Therefore, we hypothesize that myelin fragmentation results in the vesiculation of the whole compact myelin stack before the degenerated myelin becomes degraded by microglia or macrophages. 
$\mathbf{A}$
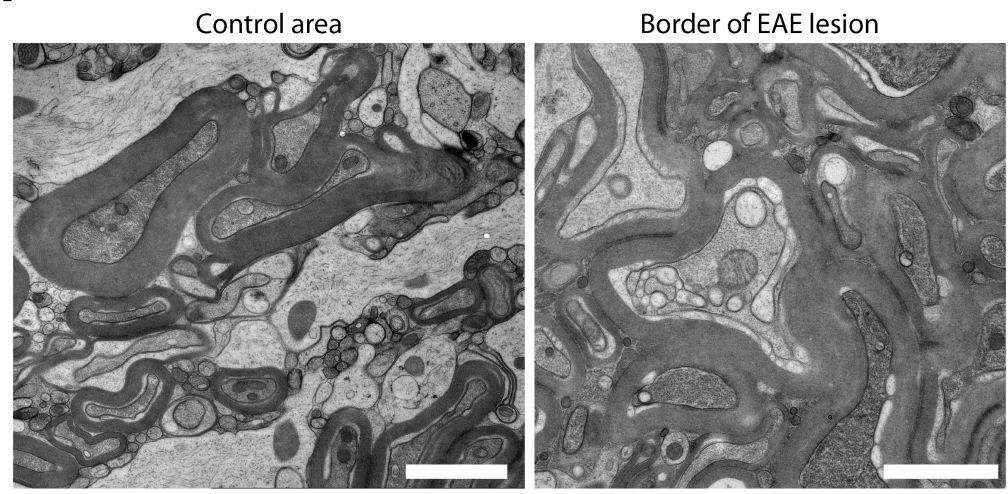

B

Intact

$$
\text { Enlarged inner }
$$

Vesiculated

Split and

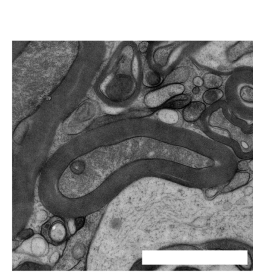

tongue sheath
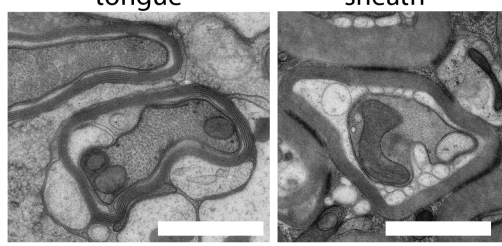
vesiculated sheath

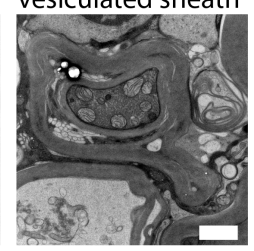

C

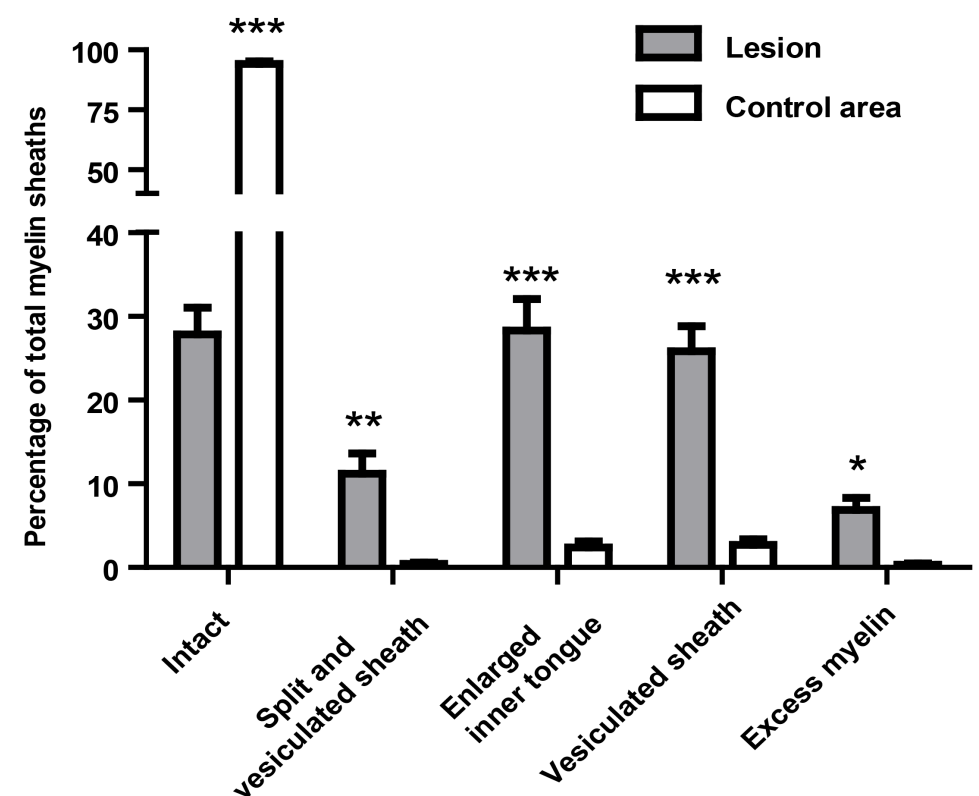

Figure 3.5: Biozzi EAE lesions during first relapse show signs of myelin pathology with enlarged inner tongue and vesiculation of the innermost layers of myelin

Representative images of cross-sections of Biozzi spinal cord in first relapse phase of EAE are shown in A. Left panel displays control area with normal myelin ultrastructure, whereas right panel displays myelin fragmentation at the border of demyelinated EAE lesion (scale bar $500 \mathrm{~nm}$ ). Myelin fragmentation profiles quantified are depicted in B, whereas the percentage of all different fragmentations is shown in $\mathrm{C}$ for the border of EAE lesion and control area. Bars display mean with SEM ( $\mathrm{n}=5$ animals, $>300$ axons per animal, $*=\mathrm{P}<0.05, * *=\mathrm{P}<0.01, * * *=\mathrm{P}<0.001$, one-way ANOVA). 


\subsubsection{Loss of MBP from vesiculated myelin in early NMO lesions}

So far MBP is the only protein known to be necessary for compaction and hence, we aimed to assess whether this protein is displaced from the membrane before the lamellae become vesiculated. As the focal NMO models induced the most uniform, synchronized and widespread pattern of myelin degeneration, we processed the lesions after $18 \mathrm{~h}$ p.i. for cryo-immunoelectron microscopy. The cryosections were labeled with a polyclonal MBP antibody and the labeling was quantified by calculating a ratio of gold particles detected in the compact myelin to the ones found in the inner tongue including the vesicles obtained upon AQP4 antibody injection. Hardly any MBP labeling was obtained in the inner tongue and the vesiculated myelin, whereas robust labeling was found in the compact membrane stacks for both conditions (see Figure 3.6 A upper panel). This results in a ratio that did not display any differences between the PBS injected control and the NMO lesion (see Figure $3.6 \mathrm{~B}$ ). Hence, the distribution of MBP upon AQP4 antibody injection does not seem to change as it can be only detected in the compact myelin and not in the vesiculated membrane. This suggests that the vesicules of myelin are devoid of MBP.

In order to assure that the vesicules detected at the inner tongue are derived from the membrane of the compact myelin, we also labeled the control and AQP4 lesions with PLP. The most abundant myelin protein is a transmembrane protein that should be contained within the vesicles, if they are derived from compact myelin. By using PLP as a second marker, we aimed to rule out that a poor antibody penetration was the cause of the lack of labeling for MBP.

Our results indicate that the ratio of labeling in the compact myelin to the staining in the inner tongue was dramatically changed in NMO lesions (see Figure $3.6 \mathrm{C}$ ). The vesiculated membrane at the inner tongue was heavily labeled with PLP, whereas hardly any labeling was detected in the adaxonal space of PBS injected animals (see Figure 3.6 A lower panel). Thus, PLP, which is normally not detected in the inner tongue is contained in the vesicules found in the inner tongue upon AQP4 antibody injection. These results lead to the conclusion that the vesiculated membrane is derived from compact myelin and contains PLP, one of the most abundant myelin proteins.

In conclusion, these results suggest that the vesicules obtained upon AQP4 antibody injection contain PLP, but are devoid of the abundant compact myelin protein MBP. Hence, the peripheral MBP seemed to have lost its ability to adhere to the myelin membrane and this might results in myelin vesiculation and subsequent, degradation of the myelin layers.

Supportive for this hypothesis is also that the myelin membranes detected in 
shiverer mice (MBP deficient mutant) form only a few uncompacted wraps and extend several microprocesses around the axon prior to vesiculation and loss of myelin (see Figure 5.8). Hence, the myelin membrane seems to be unstable without the compaction by MBP highlighting its crucial role in myelin maintenance.

A
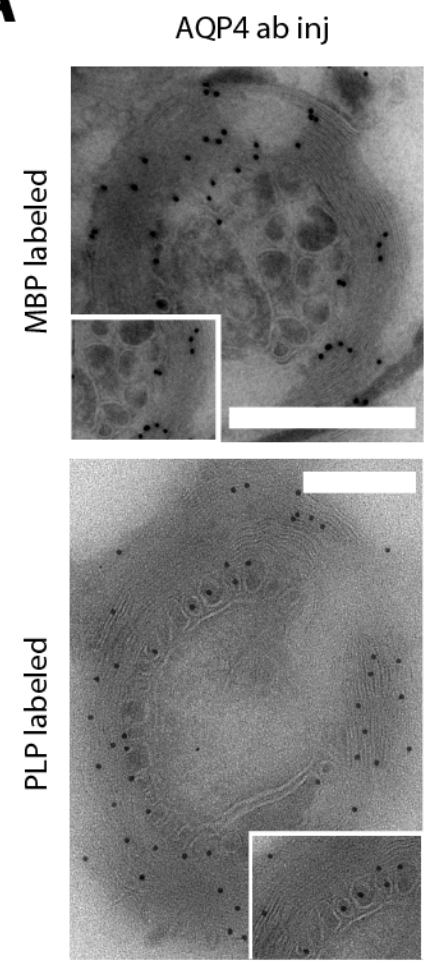
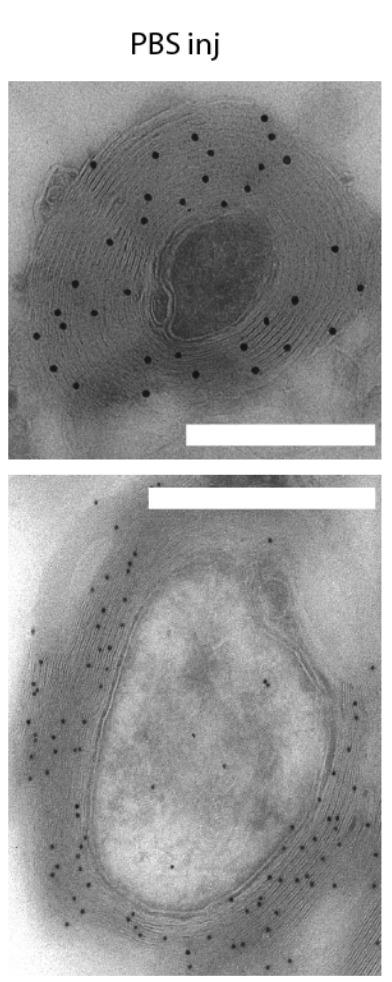

B
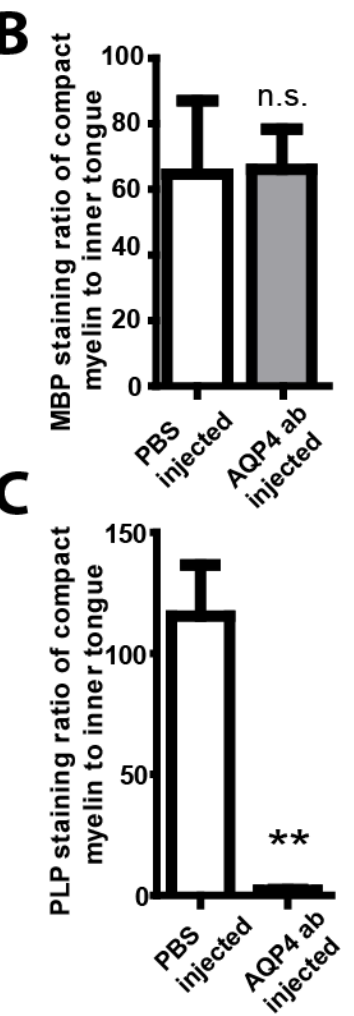

Figure 3.6: Vesicules of inner myelin layers in early NMO lesions consist of myelin membrane, but lack MBP

A. Human AQP4 antibody or PBS and complement was injected in the corpus callosum of adult Lewis rats. With immunoelectron microscopy, we determined the subcellular localization of MBP (upper panel) and PLP (lower panel) at $18 \mathrm{~h}$ p.i. (scale bar $500 \mathrm{~nm}$, gold size $15 \mathrm{~nm}$ for MBP, $10 \mathrm{~nm}$ for PLP). The vesicles at the inner tongue are enlarged in the white boxes. Quantification of MBP (B) and PLP (C) labeling distribution within the inner tongue (in PBS injection) or inner tongue with vesicles (for AQP4 ab injection). Bars shown mean with SEM ( $\mathrm{n}=3$ animals, $>70$ axons per animal, ${ }^{* * *}=\mathrm{P}<0.001$, Student's t-test).

\subsection{Reverse phase transition of MBP}

MBP is a naturally unstructured protein, which acquires an extended Cshape with a high degree of secondary structure only upon binding to the 


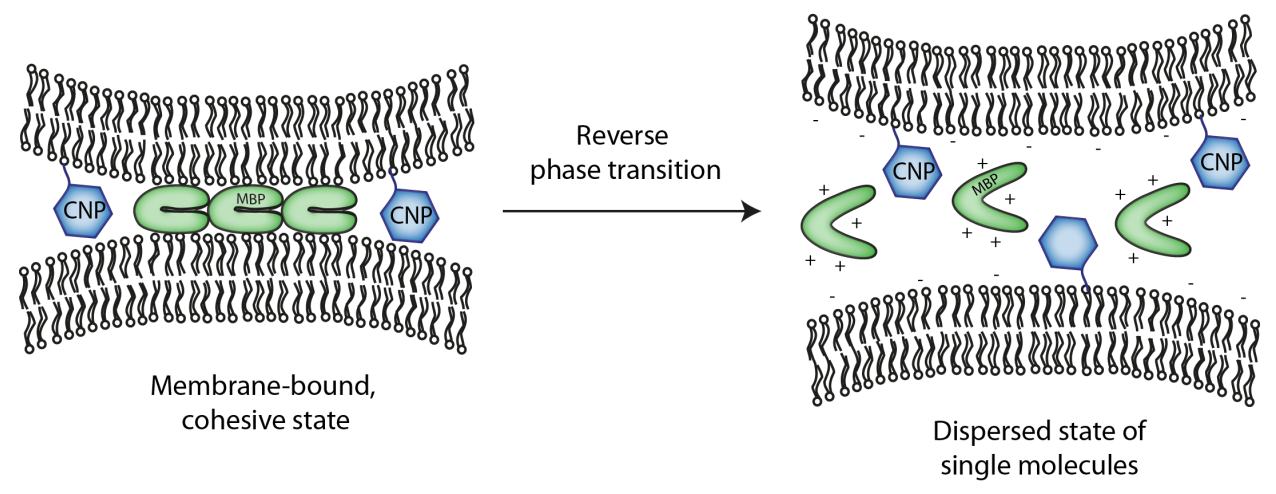

Figure 3.7: Reverse phase transition of MBP

The schematic drawing depicts MBP in its cohesive state after binding to negatively charged lipids in the membrane (on the left), where it carrys out its extrusion function of proteins with a spacious cytosolic domain (represented by blue hexagon). On the right hand side, MBP is shown in a dispersed state after detaching from the membrane and losing its self-interacting ability. Hence, MBP is not able to extrude proteins any longer.

negatively charged lipids in the myelin membrane. This conformational change allows MBP to self-interact and carry out its compaction function. Such phase transition of macromolecules generate liquid-liquid separation leading to distinct compartments with slowed diffusion and non-linear signaling pathways [343]. For example, nuclear pore complexes proteins that are intrinsically unfolded and contain phenylalanine-glycine (FG)-rich repeats were shown to form a sieve-like hydrogel that restricts the entry of molecules of a certain size into the nucleus. Mutations of phenylalanine $\rightarrow$ serine $(\mathrm{F} \rightarrow \mathrm{S})$ in the repeats blocked the hydrogel formation. The generated selective phase is important for restricting inert molecules from entering the nucleus, whereas these hydrophobic interactions were shown to break the mesh-like network [344] [345].

We hypothesize MBP also undergoes a phase transition upon binding to the membrane as it also restricts the entry of proteins with a bulky cytosolic domain to the compacted areas. Moreover, we suggest that upon changes in the lipid environment or in the ion homeostasis, MBP can revert from its cohesive membrane-bound network into a dispersed state of single soluble molecules (see Figure 3.7).

In order to prove this reverse phase transition, we need to design a tool that can detect these different MBP states.

To be able to develop these tools, we investigated the molecular interactions of MBP at the protein-protein and protein-lipid level. These molecular interactions might be mediated by specific domains that become unmasked upon disintegration of the MBP network. 
In order to assess which features of $\mathrm{MBP}$ are relevant for the molecular interaction of MBP with the membrane and/or with itself, we investigated the role of the amphipathic helices that were previously shown to play a role in the assembly of the MBP network. The amphipathic helices have two faces: one necessary for the binding to the membrane and one facing towards the cytoplasm. Aggarwal et al. (2013) showed that the phenylalanines within the amphipathic helices are not necessary for the binding to the lipids, but for the self-interaction of MBP [28] [10].

Therefore, we generated single mutants of the phenylalanines in the two amphipathic helices of the $14 \mathrm{kD}$ isoform (for localization of phenylalanines, see 3.15), the most prevalent isoform in the mouse brain, to further determine which of these amino acids are essential to the function of MBP. Using site-directed mutagenesis, we obtained the following mutants:

$$
\begin{gathered}
-\mathrm{F} 1,6 \rightarrow \mathrm{S} \\
-\mathrm{F} 2,3 \rightarrow \mathrm{S} \\
-\mathrm{F} 4,5 \rightarrow \mathrm{S} \\
-\mathrm{F} 2,3,4,5 \rightarrow \mathrm{S}
\end{gathered}
$$

We then expressed these plasmids in bacteria to obtain the recombinant proteins in order to investigate the function of these mutants in a range of biochemical and biomimetic assay explained in detail in the next paragraphs.

\subsubsection{Liposomes binding assay with $\mathrm{MBP} \mathrm{F} \rightarrow \mathrm{S}$ mutants}

To determine whether the mutants are still capable of binding their normal membrane environment, we employed liposomes of inner leaflet composition of myelin membranes (ILC) to resemble their in vivo binding conditions and carried out a in vitro binding assay.

Therefore, liposomes of ILC were incubated with the recombinant MBP proteins leading to crosslinking of liposomes via MBP. Fractions of the crosslinked liposomes were visualized in EM by negative staining. We could show that all MBP mutants were able to crosslink the membrane and form clusters with liposomes (for representative images for $\mathrm{WT} \mathrm{MBP}$ and $\mathrm{MBP} F \rightarrow \mathrm{S}$ see Figure $3.8 \mathrm{~B})$.

After allowing the protein to crosslink the liposomes, the aggregates were pelleted using ultracentrifugation. Fractions of the pellet and the supernatant were employed to perform Western Blotting for MBP protein. This revealed that the WT MBP, as well as the mutants were found in the pellet (for representative blots of the WT and the $\mathrm{F} \rightarrow \mathrm{S}$ mutant see Figure 3.8 A). Hence, we could conclude that even the mutants retain their membrane binding ability in case of liposomes with ILC. For liposomes with ILC lacking cholesterol, all proteins were still retained in the pellet fraction. In case of liposomes of ILC without PS and PIP2, we found all proteins in the su- 
pernatant; hence, they could not bind the membrane anymore. Thus, this is clear evidence that the electrostatic interactions with the membrane are necessary - at least - for initial binding. In conclusion, all $\mathrm{F} \rightarrow \mathrm{S}$ mutants generated are still capable of binding the membrane in presence of negatively charged lipids.

A
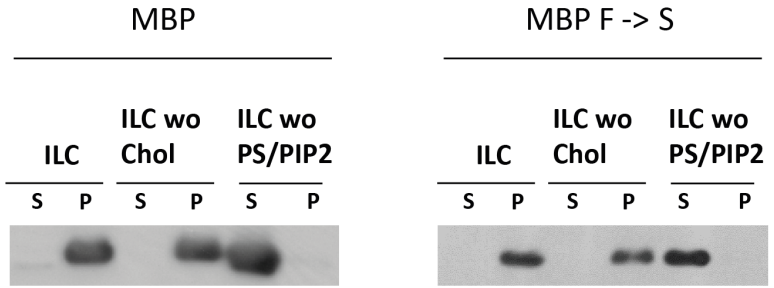

B

Liposomes only

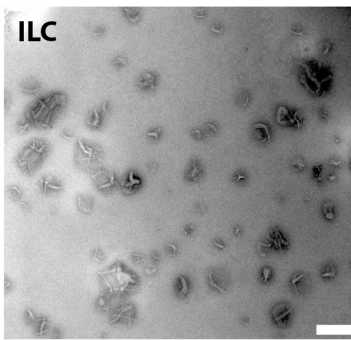

\section{ILC without Cholesterol}

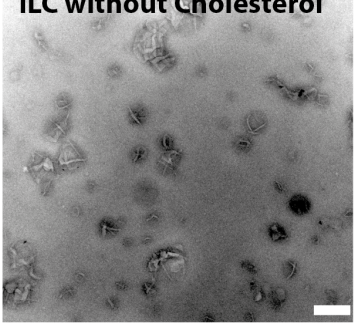

MBP with Liposomes
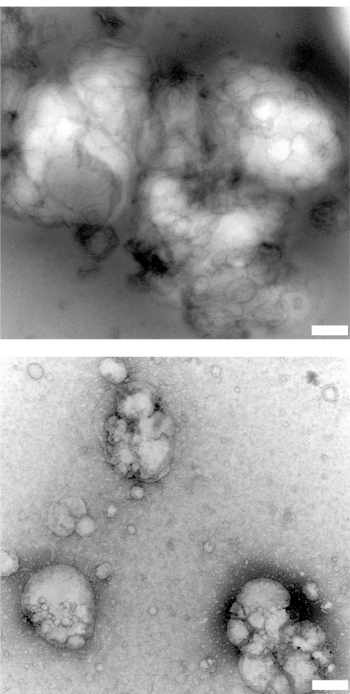

MBP F->S with Liposomes
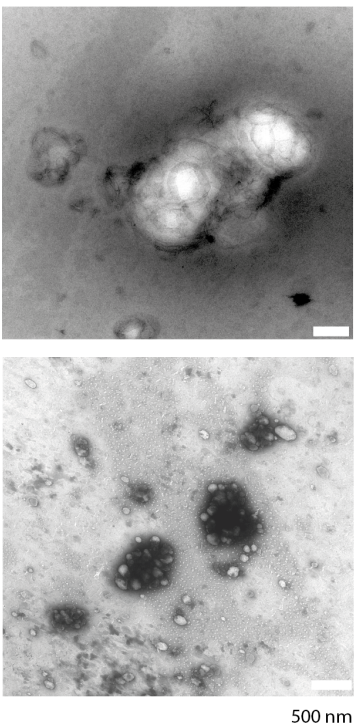

Figure 3.8: All $\mathrm{F} \rightarrow \mathrm{S}$ mutants are capable of binding the membrane and crosslinking liposomes

When liposomes are incubated with recombinant $14 \mathrm{kD}$ MBP proteins, these crosslinked liposomes can be pelleted via ultracentrifugation. Fractions of the pellet and supernatant can then indicate the binding of the proteins to the liposomes. MBP and $\mathrm{F} \rightarrow \mathrm{S}$ mutants crosslink liposomes of inner leaflet composition (ILC) with or without cholesterol, but not devoid of the negatively charged lipids, PS and PIP2 (A). These results were confirmed using negative EM (B), where the crosslinked liposomes of inner leaflet composition with or without cholesterol were detected for the $\mathrm{WT} \mathrm{MBP}$, as well as for the $\mathrm{F} \rightarrow \mathrm{S}$ mutant (scale bar $500 \mathrm{~nm}$ ). 


\subsubsection{Spectroscopy of WT peptides of amphipathic helices}

MBP is a naturally unstructured protein that acquires its three dimensional structure only upon interaction with the membrane. A high resolution structure cannot be determined as MBP cannot be crystallized. Therefore, we carried out various techniques of circular dichroism (CD) spectroscopy in collaboration with Prof. Petri Kursula (at the University of Bergen, Norway) to determine, if the phenylalanine residues within the amphipathic helices are required for the conformational change and the function of the helix.

CD spectra of WT peptides of the amphipathic helices (MBPp1 (sequence: QDENPVVHFFKNIV); MBPp2 (sequence: GILDSIGRFFSGDRGAPKR)) in membrane mimicking conditions using the detergent dodecyl phosphatidyl choline (DPC) revealed that these peptides acquire a helical conformation. In contrast to that, the $\mathrm{F} \rightarrow \mathrm{S}$ mutant peptides (MBPp1m (sequence: QDENPVVHSSKNIV); MBPp2m (sequence: GILDSIGRSSSGDRGAPKR)) remained unfolded (see Figure 3.9A). Similar results were obtained when peptides were mixed with lipid vesicles of $\mathrm{PC} / \mathrm{PG}$ in 1:1 ratio and SRCD spectra were recorded (see Figure 3.9B). Hence, the interaction of phenylalanines with the membrane seem to induce a structural change of the amphipathic helix.

To further define the orientation of the helical peptides within the membrane, OCD spectra in oriented multilayers of PC/PG were acquired. The results indicated that MBP peptide 1 (MBPp1) obtains a tilted conformation within the membrane as suggested by a loss of the peak at $208 \mathrm{~nm}$ (see Figure 3.9B). In contrast to that, MBP peptide 2 (MBPp2) seems to remain parallel to the membrane as indicated by the strong band at $208 \mathrm{~nm}$. Further experiments, in which the head group charge or the hydrophobic tail length was varied did not yield any effect.

Taken together, these results indicate that the specific interactions of the phenylalanine residues with the membrane are required for the structural change of the amphipathic helix. 
a

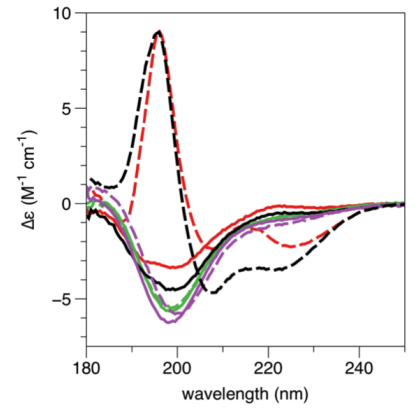

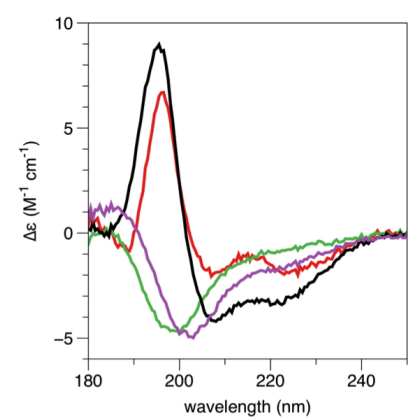

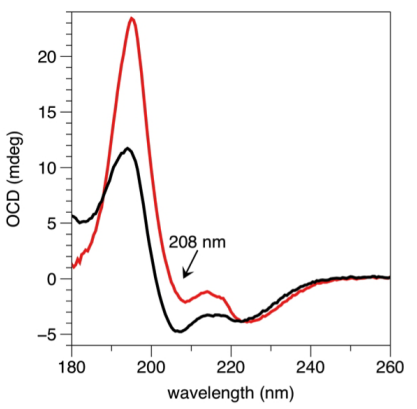

Figure 3.9: Phenylalanines mediate structural change of MBP peptides CD spectroscopic analysis of peptides mimicking the $14 \mathrm{kDa}$ MBP amphipathic helices. A.) Conventional CS spectra of peptides in absence (solid lines) or presence (dashed lines) of $0.1 \%$ DPC. MBPp1 in red (sequence: QDENPVVHFFKNIV); MBPp2 in black (sequence: GILDSIGRFFSGDRGAPKR), as well as peptides with $\mathrm{F} \rightarrow \mathrm{S}$ substitutions at these motifs: MBPp1m in green (sequence: QDENPVVHSSKNIV); MBPp2m in magenta (sequence: GILDSIGRSSSGDRGAPKR) B.) SRCD spectra of peptides mixed with lipid vesicles composed of PC/PG in 1:1 ratio. Same colours as above. C.) OCD spectra of oriented multilayers of PC/PG with peptides MBPp1 (red) and MBPp2 (black). MBPp1 acquires a tilted conformation as indicated by the loss of the peak at $208 \mathrm{~nm}$ (see arrow). Data acquired in collaboration with Arne Raasakka and Prof. Petri Kursula at the University of Bergen, Norway.

\subsubsection{Lipid droplet assay using $\mathrm{MBP} \mathrm{F} \rightarrow \mathrm{S}$ mutants}

After ensuring that the $\mathrm{F}$ to $\mathrm{S}$ mutants are still capable of binding the membrane, we aimed to investigate the functional consequences of these mutations. The first assay to assess the self-interaction of the MBP proteins is the liquid droplet assay. This assay is solely based on the charge neutralization of the highly basic MBP protein. Thus, we dissolved the protein at a high concentration of $10 \mathrm{mg} / \mathrm{mL}$ in water and then, neutralized the charge using $\mathrm{NaOH}$. In case of WT protein, $\mu \mathrm{m}$-sized droplets were observed in phase contrast microscopy (see Figure 3.10 upper panel). For the MBP mutants, where only two phenylalanines were exchanged $(\mathrm{F} 2,3 \rightarrow \mathrm{S}$ and $\mathrm{F} 4,5 \rightarrow \mathrm{S}$ ) droplets were also obtained (see Figure 3.10 middle panel). When four or all phenylalanines were exchanged $(\mathrm{F} 2,3,4,5 \rightarrow \mathrm{S} ; \mathrm{F} \rightarrow \mathrm{S})$, we were unable to observe any droplets (see Figure 3.10 lower panel). Hence, four phenylalanines seem to be necessary to mediate self-interaction of MBP. 

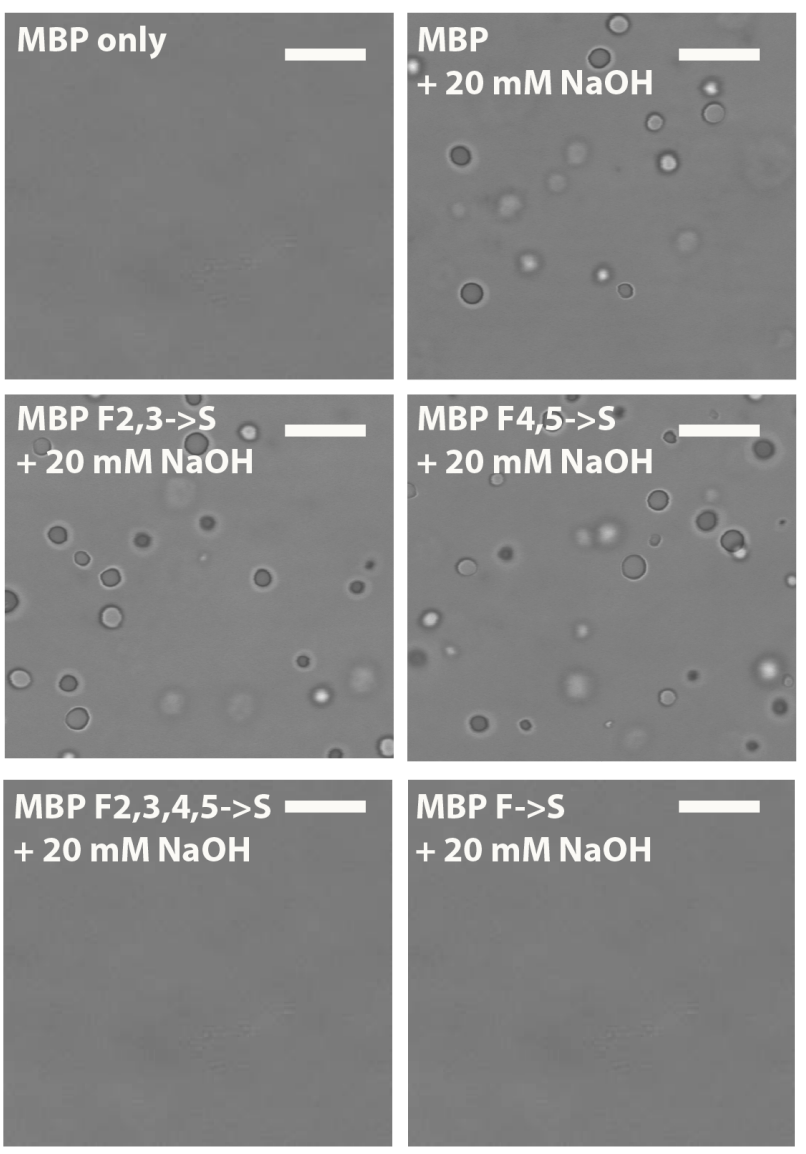

Figure 3.10: Mutations of four phenylalanines abolishes MBP droplet formation Recombinant WT MBP protein $(5 \mathrm{mg} / \mathrm{mL})$ forms $\mu \mathrm{m}$ sized droplets only in basic solution in phase contrast (upper panel). $\mathrm{F} 2,3 \rightarrow \mathrm{S}$ and $\mathrm{F} 4,5 \rightarrow \mathrm{S}$ also form droplets (middle), whereas for $\mathrm{F} 2,3,4,5 \rightarrow \mathrm{S}$ and $\mathrm{F} \rightarrow \mathrm{S}$ no droplet formation was observed (lower panel) (scale bar $10 \mu \mathrm{m})$.

\subsubsection{SLB-MBP GUV assay with MBP $\mathrm{F} \rightarrow \mathrm{S}$ mutants}

In order to further assess the self-interaction of MBP in its natural lipid environment, a biomimetic assay employing a single supported lipid bilayer (SSLB) of inner leaflet composition (ILC) and giant unilamellar vesicles (GUV's) was used.

In brief, small unilamellar vesicles (SUV's) of ILC were spread to form the SSLB. Afterwards, the MBP protein or its mutants were allowed to adhere and thereafter, GUV's composed of phosphatidylcholine/phosphatidylserine (PC/PS) were added (see Figure $3.11 \mathrm{~A}$ ). In case of the WT protein, the self-interaction of MBP induces a friction in the GUV's that causes their bursting and spreading on the coverslip (for representative images see Fig- 
ure $3.11 \mathrm{~B}$ ). If no protein was employed in this assay, the GUV stayed intact (for representative images, see Figure $3.11 \mathrm{~B}$ ). For the MBP WT protein, the GUV's bursted almost to $100 \%$, whereas the mutants that have an exchange of two phenylalanines $(\mathrm{F} 2,3 \rightarrow \mathrm{S}$ and $\mathrm{F} 4,5 \rightarrow \mathrm{S})$ had a slightly decreased rate of bursted GUV's (see Figure $3.11 \mathrm{C}$ ). For the mutants that had four or more phenylalanines were exchanged, we could observe a significant reduction of GUV bursting. This result further supports our hypothesis that four phenylalanines are necessary for the self-interaction of MBP in the presence of lipids.

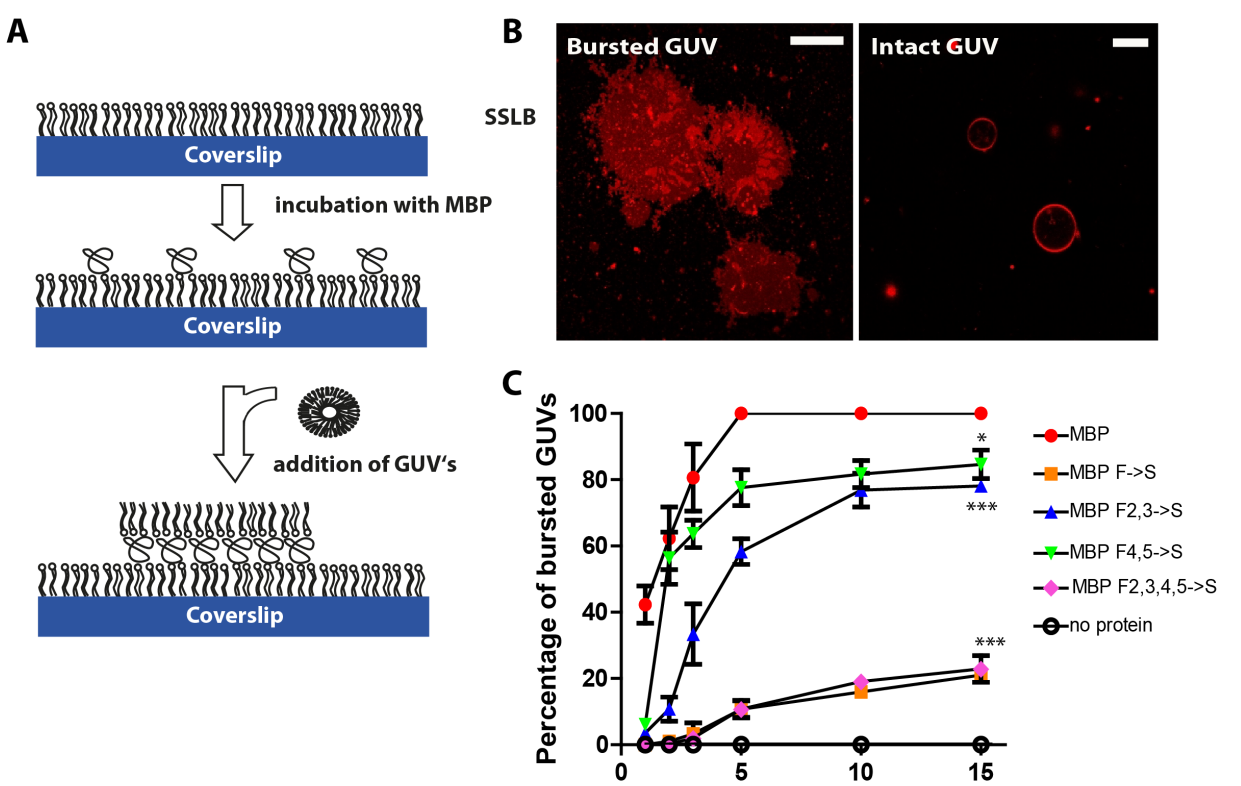

Figure 3.11: Four phenylalanines in MBP are necessary to mediate GUV spreading The left panel shows a scheme of the biomimetic SLB-MBP-GUV spreading assay: Coverslips are coated with single supported lipid bilayers (SSLB) of inner leaflet composition (44\% cholesterol, $27 \%$ PE, $2 \%$ PIP2, $11.5 \%$ PC, $12.5 \%$ PS, $3 \%$ SM) followed by incubation with $7 \mu \mathrm{M}$ recombinant MBP protein. Subsequently, GUV composed of PS and PC (1:2 ratio) are added. The WT protein induces spreading of the GUVs (see bursted GUV in $\mathrm{B}$ ), whereas the $\mathrm{F} 2,3,4,5 \rightarrow \mathrm{S}$ or $\mathrm{F} \rightarrow \mathrm{S}$ mutants do not have this effect (see intact GUV in B) (scale bar $10 \mu \mathrm{m}$ ). For every mutant employed, the percentage of GUVs bursted over time was quantified and is depicted in C.

\subsubsection{Transfection of PtK2 cells with $\mathrm{MBP} F \rightarrow \mathrm{S}$ mutants}

To further assess the self-interaction in vitro, we transfected the MBP $\mathrm{F} \rightarrow \mathrm{S}$ mutants linked to a transmembrane domain and labeled with GFP in the epithelial cell line PtK2. Upon transfection, the transmembrane domain 4 of PLP is retained in the cortical endoplasmatic reticulum (ER) with GFP in 
the lumen of the ER and MBP compacting the plasma and the ER membrane (see schematic drawing in Figure 3.12). Therefore, MBP polymerized and forms sheet-like domains as depicted in Figure $3.12 \mathrm{~B}$ on the left hand side. The domains were observed also for the $\mathrm{F} 2,3 \rightarrow \mathrm{S}$ and $\mathrm{F} 4,5 \rightarrow \mathrm{S}$ mutants, whereas upon expression of the $\mathrm{F} 2,3,4,5 \rightarrow \mathrm{S}$ mutant or $\mathrm{F} \rightarrow \mathrm{S}$ mutant this domain formation was abolished (see Figure $3.12 \mathrm{~B}$ on the right hand side). In conclusion, the phenyalanines in the amphipathic helices (F2,3 and F4,5) seem to be critical for the self-interaction of MBP into a polymer.

A

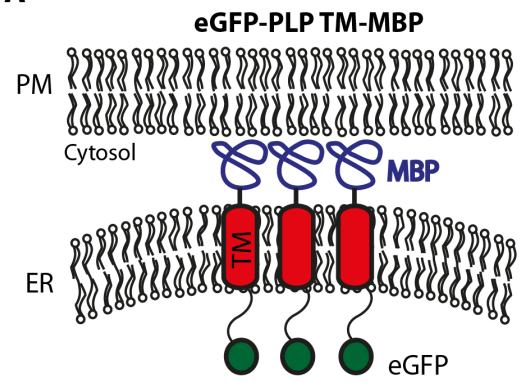

B

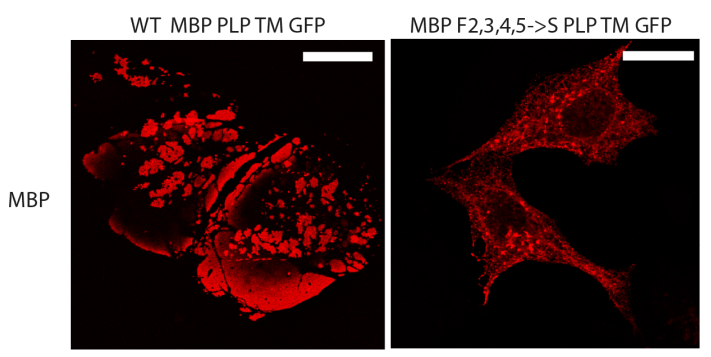

Figure 3.12: Four phenylalanines in MBP mediate domain formation in PtK2 cells PtK2 cells were transfected with plamids containing an MBP attached to a transmembrane domain of PLP (TM) and eGFP that leads to an apposition of the plasma membrane (PM) and the membrane of the endoplasmatic reticulum (ER) as depicted schemetically in A. For WT MBP, as well as F2,3 $\rightarrow \mathrm{S}$ and $\mathrm{F} 4,5 \rightarrow \mathrm{S}$ sheet-like domains were observed as shown in a representative image on the left side in $\mathrm{B}$. For $\mathrm{F} 2,3,4,5 \rightarrow \mathrm{S}$ and $\mathrm{F} \rightarrow \mathrm{S}$, no domain formation was observed as shown in the right panel in B (scale bar $25 \mu \mathrm{m}$ ).

\subsubsection{Transfection of primary mouse oligodendrocytes with MBP $\mathrm{F} \rightarrow \mathrm{S}$ mutants}

Finally, we aimed to test the integration of the $\mathrm{F} \rightarrow \mathrm{S}$ mutants in sheaths of primary WT mouse ODC's. Primary mouse ODC's were used as a wellestablished 2D model of the myelin sheath that is easy to manipulate and nevertheless displays the essential features of myelin. The branches protruding from the cell body can be stained for CNP and these so-called processes resemble feature of the non-compact myelin in vivo. The MBP positive areas in between the processes that are devoid of cytoplasm and protein with a bulky cytosolic domain [28] display similar characteristics as compact myelin in vivo with proper protein/lipid separation [346] and are termed sheets. In this study, we only used mature ODC's (days in vitro (DIV) 4 to 5) that can be stained with MBP in the sheets and CNP in the processes and thus, display features of compaction and extrusion. 

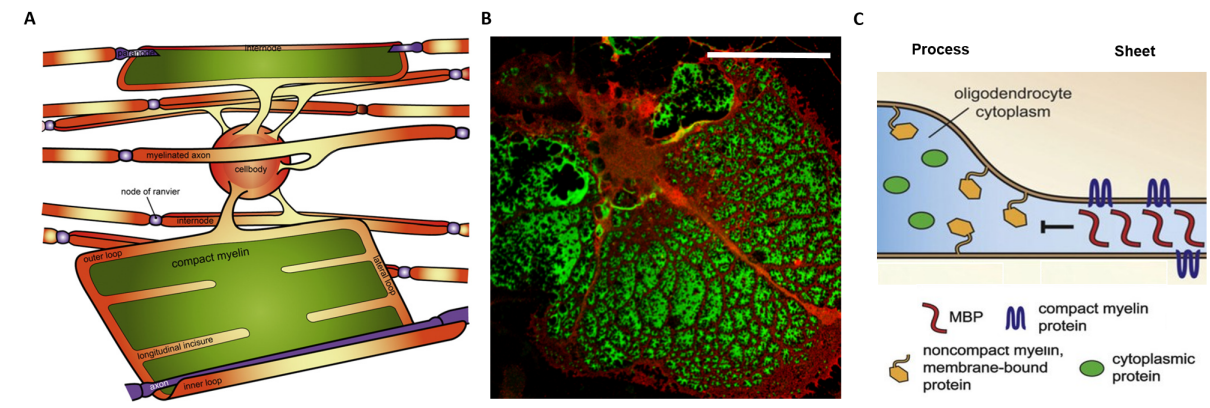

Figure 3.13: Primary mouse oligodendrocytes as in vitro model system The compartmentalization of myelin in vivo is schematically depicted in A with the compact myelin in green and the non-compacted areas in red. B shows a primary mouse oligodendrocyte stained with MBP in green and CNP in red. The MBP-positive areas, the so called sheets, ressemble compact myelin in vivo due to their close apposition of the plasma membrane in vitro as indicated in $\mathrm{C}$. This results also in the extrusion of proteins with a spacious cytosolic domain to the non-compacted area of the cells named processes. These processes resemble non-compact myelin in vivo. Figure modified from [10].

To test the localization of the $\mathrm{F} \rightarrow \mathrm{S}$ mutants in these primary cells, the plasmids encoding for the MBP WT proteins or its mutants were transfected into ODC's on DIV 3. Sixteen hours after transfection, the cells were stained for total MBP and the modified MBP via the myc-tag of the transfected construct (see Figure $3.14 \mathrm{~A}$ ). Results of this targeting assay revealed that the WT MBP and the mutants containing only two mutated phenylalanines were still incorporated into the sheets suggesting that they are still capable of self-interaction. Strikingly, the mutants with four or more mutated phenylalanines were retained in the processes and failed to be incorporated into the sheets of the ODC's.

Taken together all data from the biochemical, biomimetic and in vitro studies suggest that the MBP $\mathrm{F} \rightarrow \mathrm{S}$ mutants with at least 4 amino acids changes exhibit a normal membrane binding, but display an impaired self-interaction and therefore, a loss of function-variant of the MBP protein. Hence, the hydrophobic interaction of the amphipathic helices of MBP with the lipids in the bilayer are crucial for the proper function of the protein. 


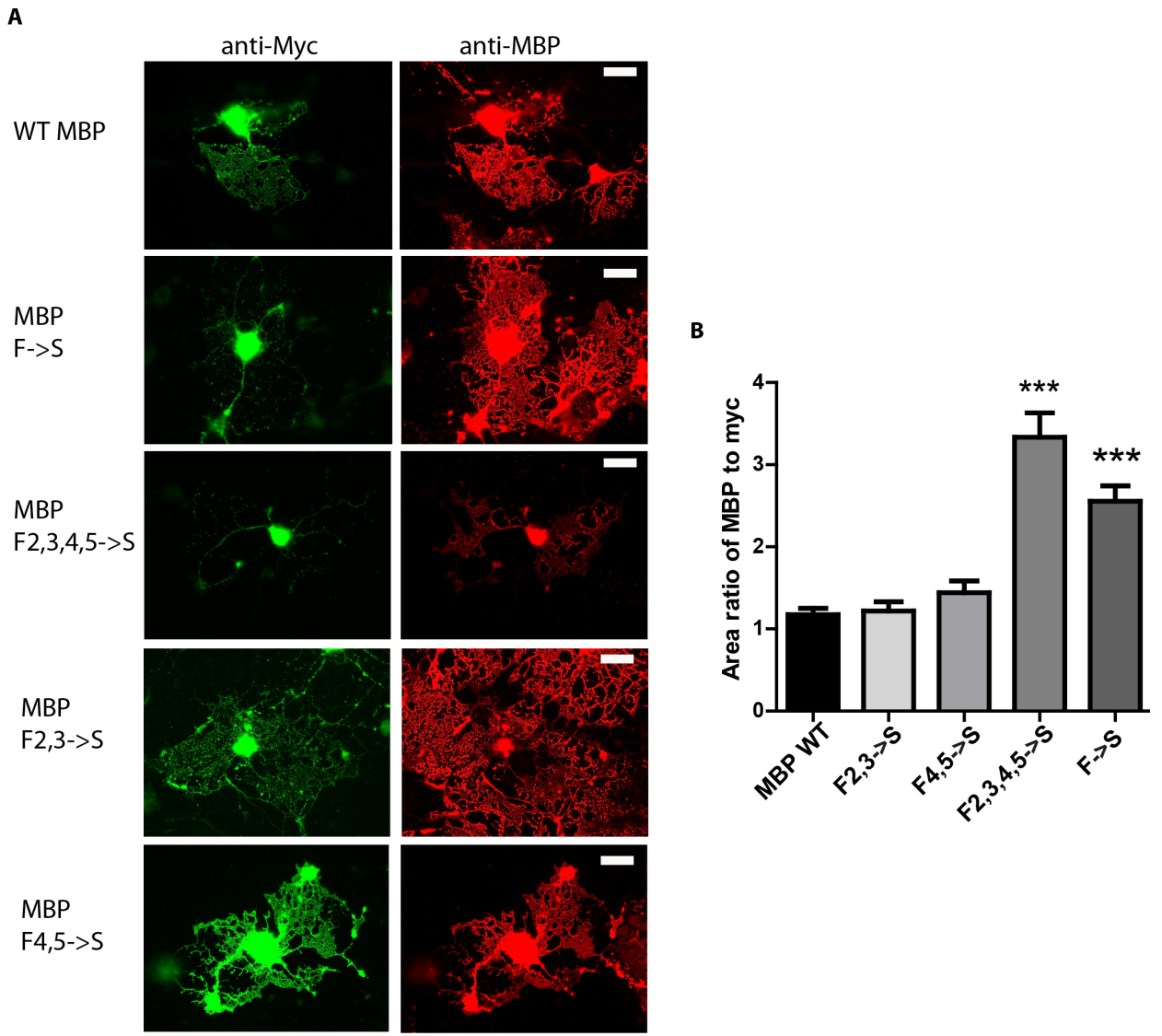

Figure 3.14: Four phenylalanines are necessary for the integration of MBP in sheets of oligodendrocytes

Primary mouse oligodendrocytes were transfected with WT MBP or the different $\mathrm{F} \rightarrow \mathrm{S}$ mutants at DIV 4 and subsequently stained for total MBP (in red) and the transfected plasmid linked to a myc tag (in green). For WT MBP, F2,3 $\rightarrow \mathrm{S}$ and F4,5 $\rightarrow \mathrm{S}$, the transfected constructs were targeted to the sheets, whereas the other mutants were retained in the processes (A) (scale bar $25 \mu \mathrm{m}$ ). The area occupied by MBP and myc staining was quantified in B. Bars show mean with SEM $\left(n=3,>20\right.$ cells per condition, ${ }^{* * *}=P<0.001$, one-way ANOVA).

\subsubsection{Tools to detect reverse phase transition}

We could show that the phenylalanine residues within the amphipathic helices of MBP are necessary for self-interaction of MBP and its function for compaction. In order to test whether MBP undergoes reverse phase transition, we had to develop tools to detect the different states of MBP. Due to the finding that the amphipathic helices play a role in the network formation of MBP, these helices that are normally buried within the membrane might get exposed upon disassembly of the MBP network. 
Hence, we screened several antibodies raised against epitopes within the amphipathic helices: We found an antibody named QD9 commercially available from abcam (Cambridge, UK) is raised against the human MBP residues 82-88 (marked in Figure 3.15 A in the black box). This epitope is adjacent to one of the amphipathic helices that we found to be relevant to self-association of MBP. Therefore, it might recognize the dispersed and dysfunctional state of MBP. Interestingly, Matsuo et al. (1998) showed that it specifically stains areas of degenerated myelin in Multiple Sclerosis and Multiple System Atrophy [347].

As this antibody is a potential tool to detect reverse phase transition of MBP from a cohesive state to a dispersed state, we tested the QD9 antibody in Western blots of myelin fractions of adult brains (see Figure 3.15 B). The QD9 antibody detected only full length monomeric MBP and no fragments of smaller molecular weight. The most prominent isoform of the $14 \mathrm{kD}$ MBP was the band with the highest intensity, whereas also bands at 17 and $18 \mathrm{kD}$ were stained with this antibody. Despite long exposures, employing higher amount of samples or ECL solution with high sensitivity, no band of higher molecular weight were obtained at any time. In contrast to that, a polyclonal MBP antibody (Dako Cytomat., Carpinteria, USA) detected oligomeric bands of higher molecular weight. 
A

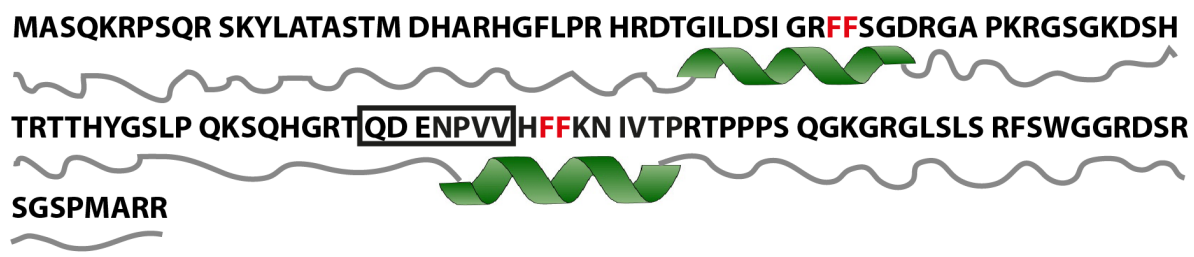

B
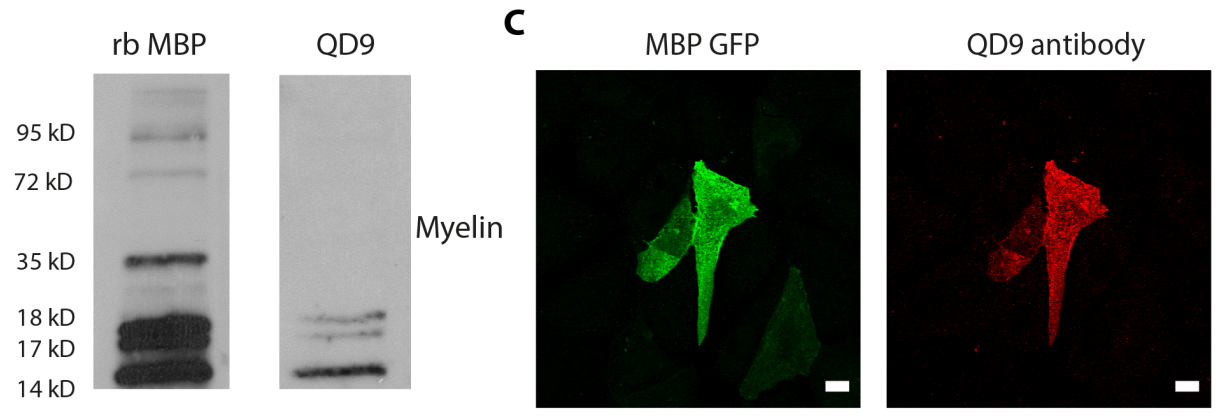

Figure 3.15: QD9 recognizes full length monomeric MBP

The QD9 antibody epitope of human MBP residues amino acid 82 to 88 is marked in the black box of the $14 \mathrm{kD}$ MBP amino acid sequence in A, whereas the amphipathic helices of MBP are marked in green underneath the sequence. B. Myelin fractions were employed for Western blotting and stained with an anti-MBP or QD9 antibody. The MBP antibody recognizes several MBP bands also of higher molecular weight, whereas QD9 only detects the monomeric MBP isoforms. In C, MBP eGFP transfected PtK2 cells were stained with the QD9 antibody (scale bar $25 \mu \mathrm{m}$ ).

Furthermore, the QD9 antibody stains MBP under non-physiological conditions, such as in soluble MBP-eGFP tagged transfected PtK2 cells (see Figure $3.15 \mathrm{C}$ ). As the amphipathic helix epitope of QD9 is normally buried within the membrane, the fixation of the cells or tissue is crucial for a good staining as these regions are easily exposed upon permeabilisation. Therefore, we fixed the cells used for this study with $4 \%$ PFA and $0.25 \%$ glutaraldeyhde. Hardly any QD9 staining was obtained in the sheets of mature primary ODC's that are positive for MBP when staining with a polyclonal MBP antibody (see control cells in Figure $3.17 \mathrm{C}$ ). Moreover, when we expressed myc-tagged MBP F2,3,4,5 $\rightarrow$ S previously shown to be retained in the processes of primary ODC's (see Figure 3.14) and stained these cells with QD9, we obtained a much more intense staining in the processes of these cells than in the sheets of ODC's that incorporated transfected WT MBP.

Taken together these results suggest that the QD9 antibody detects full length MBP under non-physiological conditions when it is not correctly assembled and seems to be able to discriminate different MBP states. Hence, we suggest that this antibody can be used as a tool to test our hypothesis 
of reverse phase transition.
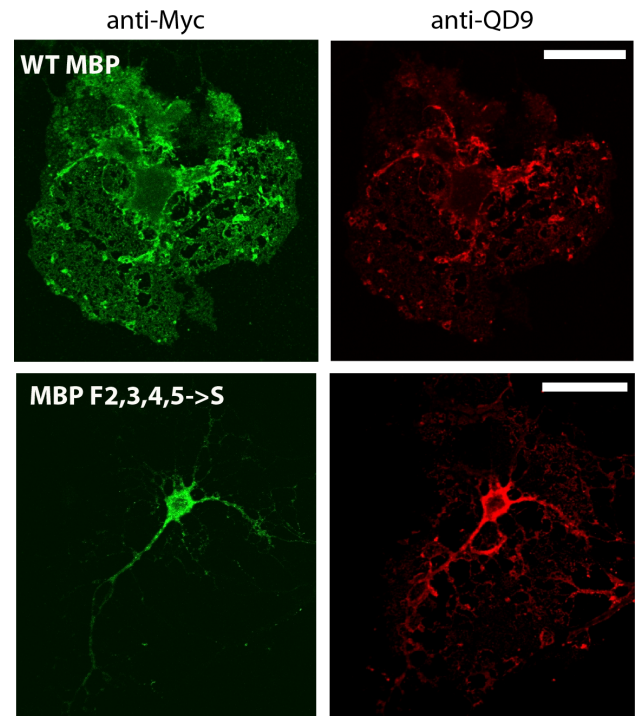

Figure 3.16: QD9 recognizes structurally aberrant MBP like $\mathrm{F} \rightarrow \mathrm{S}$ MBP mutant Primary mouse oligodendrocytes were transfected with WT MBP or the different $\mathrm{F} \rightarrow \mathrm{S}$ mutants at DIV 4 and subsequently stained for QD9 (in red) and the transfected plasmid linked to a myc tag (in green). For WT MBP, the transfected constructed was targeted to the sheets, but hardly stained with QD9. In contrast to that, the F2,3,4,5 $\rightarrow$ S mutant was retained in the processes and heavily stained there with QD9 (scale bar $25 \mu \mathrm{m}$ ).

\subsection{In vitro reverse phase transition model}

\subsubsection{Disruption of electrostatic interaction between MBP and the membrane}

In order to further test whether MBP undergoes reverse phase transition in vitro, we employed conditions previously known to affect the MBP network. MBP is known to interact with PIP2 and this interaction can be abolished by increasing intracellular calcium levels [348]. Upon treatment with the calcium ionophore Ionomycin $(10 \mu \mathrm{M}, 2$ mins $)$, MBP detaches from PIP2 in the membrane. Hence, the PIP2 epitope is unmasked and can be stained, despite being masked by strong binding of MBP under normal conditions (see Figure $3.17 \mathrm{C}$ ). Upon antagonizing of the electrostatic interactions of MBP with the membrane by treatment of ODC's with Ionomycin, MBP looses its function. This can be shown as CNP, a protein normally found in the processes of ODC's, is then found in MBP positive areas (see Figure 3.17 E). Previous studies from our laboratory have shown that MBP compacts the membranes and extrudes proteins with a big cytosolic domain, 
like CNP from these areas. Hence, a co-localization of MBP and CNP upon Ionomycin treatment indicates that MBP lost its extrusion function.

Thus, Ionomycin leads to detachment and the loss of function of MBP and hence, we stained Ionomycin treated cells with QD9 and MBP to test whether the antibody can discriminate the different MBP states. Upon increase of intracellular calcium by Ionomycin, the QD9 epitope was rapidly unmasked in the MBP positive areas. By calculating a ratio of the MBP to QD9 staining using the integrated density, we could show a significant decrease in this ratio upon Ionomycin treatment of primary ODC's. Similar results were obtained upon antagonizing of the electrostatic interactions using $100 \mu \mathrm{M}$ sphingosine for 5 mins in our ODC model as also MBP to QD9 ratio was vastly decreased (see Figure 3.18).

Collectively, these data suggest that the electrostatic interactions of MBP with the membrane are not only important for its binding, but also for executing its extrusion function. Moreover, the QD9 antibody can be used to define a non-physiological dysfunctional state of MBP in vitro upon disruption of the electrostatic interactions with the membrane. 
A

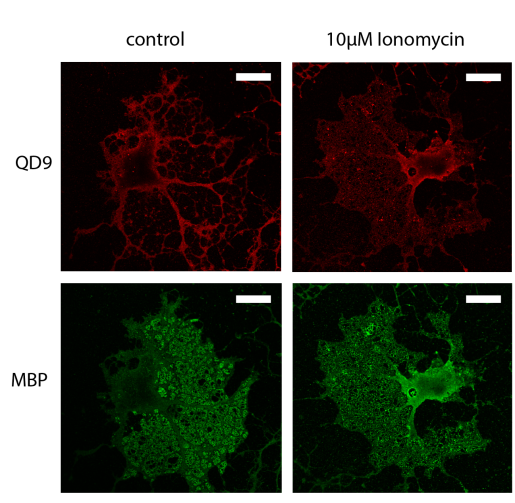

B

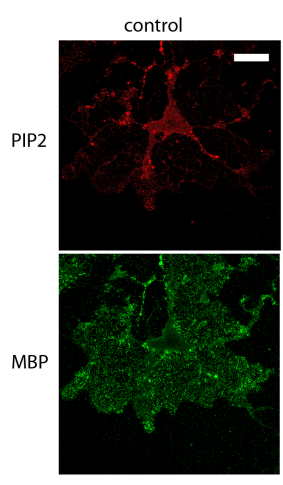

$\mathbf{E}$

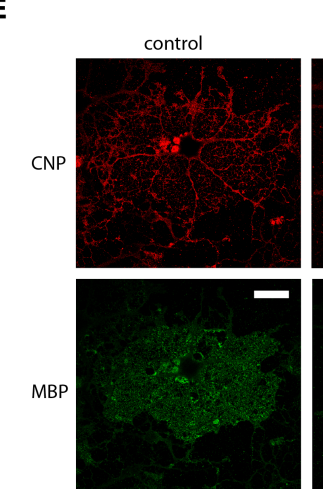

$10 \mu \mathrm{M}$ lonomycin

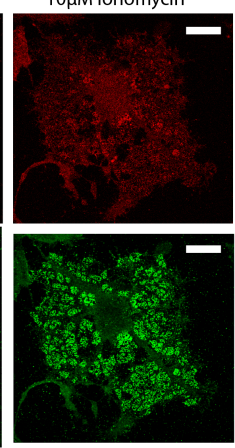

D
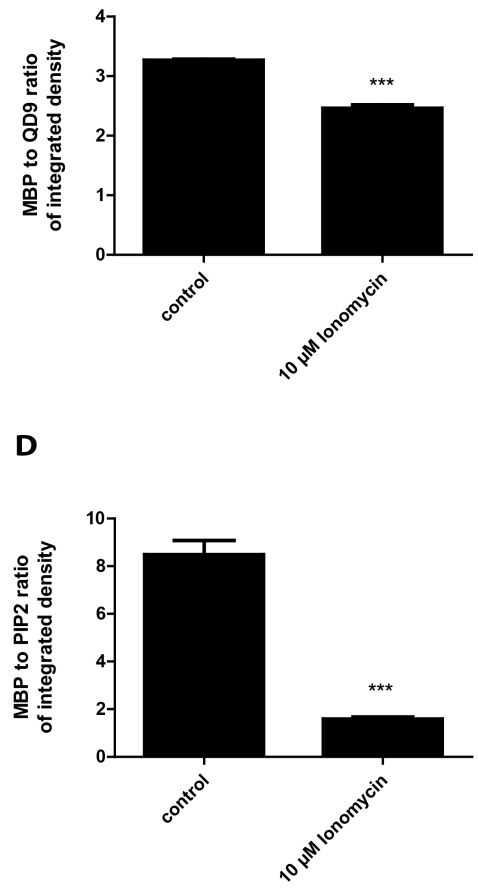

$\mathbf{F}$

$10 \mu \mathrm{M}$ lonomycin
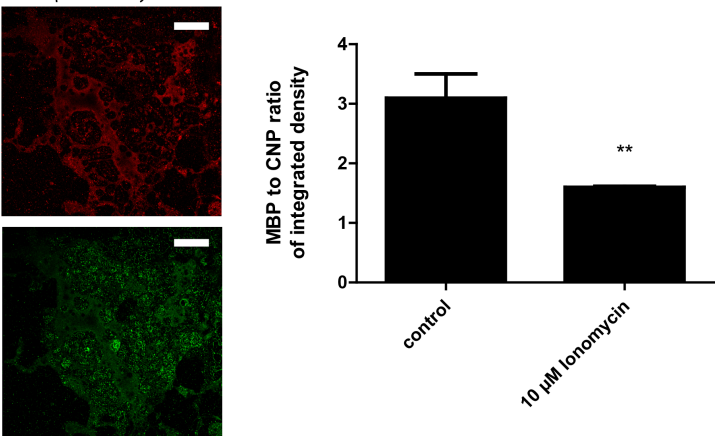

Figure 3.17: Ionomycin detaches MBP from the membrane and leads to decompaction Primary mouse oligodendrocytes at DIV 5 were treated with $10 \mu \mathrm{M}$ Ionomycin in KrebsRinger solution for 2 mins and subsequently, stained for MBP (in green) and QD9 (in red, A), or PIP2 (in red, B) or CNP (in red, C) (scale bar $25 \mu \mathrm{m}$ ). Quantification of the staining are depicted in $\mathrm{B}, \mathrm{D}$ and $\mathrm{F}$ respectively. Bars show mean with SEM $(\mathrm{n}=3$, $>20$ cells per condition, ${ }^{* *}=\mathrm{P}<0.01,{ }^{* * *}=\mathrm{P}<0.001$, Student's t-test). 
A

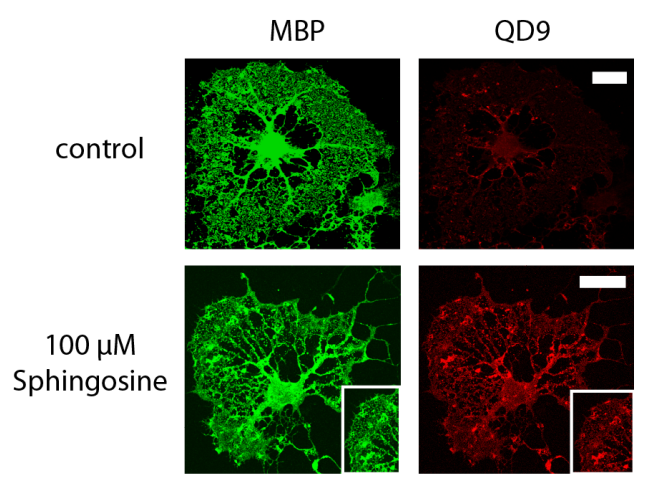

B

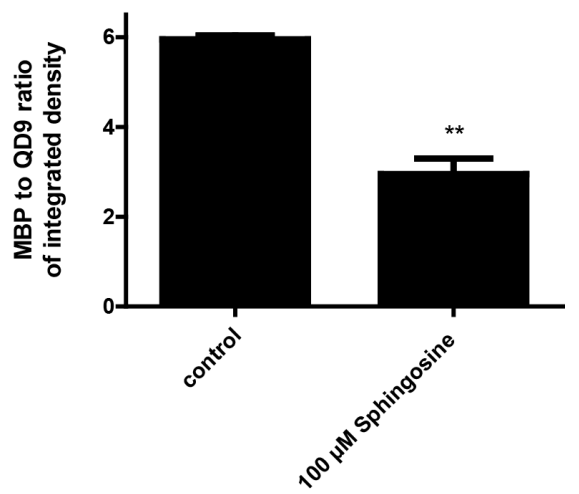

Figure 3.18: Sphingosine treatment of oligodendrocytes lead to QD9 unmasking Primary mouse oligodendrocytes at DIV 5 treated with $100 \mu \mathrm{M}$ Sphingosine for 5 mins were stained for MBP (in green) and QD9 (in red, A) (scale bar $25 \mu \mathrm{m}$ ). Quantification of the ratio of the integrated density of the MBP to QD9 is depicted in B. Bars show mean with SEM ( $\mathrm{n}=3,>20$ cells per condition, ${ }^{* *}=\mathrm{P}<0.01$, Student's t-test).

\subsubsection{Disruption of hydrophobic interaction between MBP and the membrane}

Not only electrostatic interactions, but also hydrophobic interactions with the membrane are important for the binding and function of MBP. Therefore, we employed several treatments to decrease hydrophobic lipids, such as cholesterol and ceramides within the membrane to study the effect on the adhesion and extrusion abilities of MBP in primary mouse ODC's.

Glycosphingolipids and cholesterol are an important component of the membrane microdomains, so called lipid rafts. The de novo biosynthesis of sphingolipids can be blocked by inhibition of ceramide synthase using the mycotoxin Fumonisin B1 (FB1). Spingolipids are an integral component of the myelin membrane and important for the binding of MBP. Hence, we depleted these lipids by treating primary ODC's with $50 \mu \mathrm{M}$ FB1 from d1. This lead to an unmasking of the QD9 epitope in mature ODC's and a significant decrease in the total MBP to QD9 ratio suggesting an increase in dysfunctional MBP (see Figure 3.19 A and B).

To determine the loss of compaction in FB1 treated ODC's, we stained these cells with MBP and CNP. We found focal regions where MBP and CNP colocalized in the sheets of FB1 treated cells that do not occur in control cells (see arrow in Figure $3.19 \mathrm{C}$ ). Hence, a decrease in MBP to CNP ratio could be detected in FB1 treated cells. Hence, depletion of sphingolipids leads to a loss of compaction and extrusion function of MBP and the pool of dysfunctional MBP can stained with the QD9 antibody. 
A
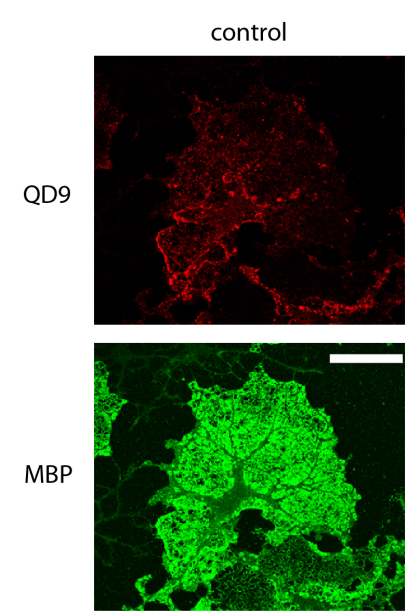

C

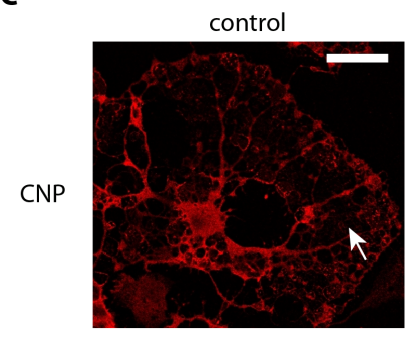

MBP

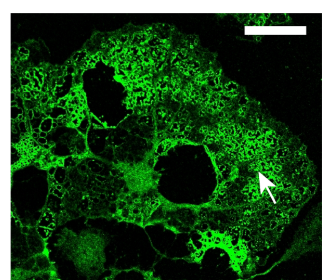

$50 \mu \mathrm{M}$ Fumonisin B1
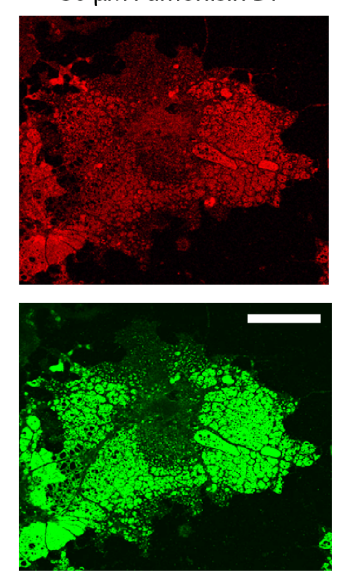

$50 \mu \mathrm{M}$ Fumonisin B1
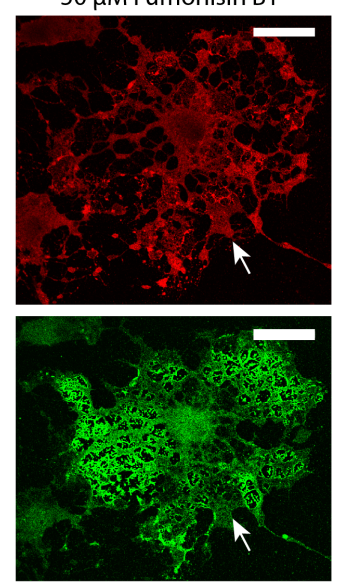

B

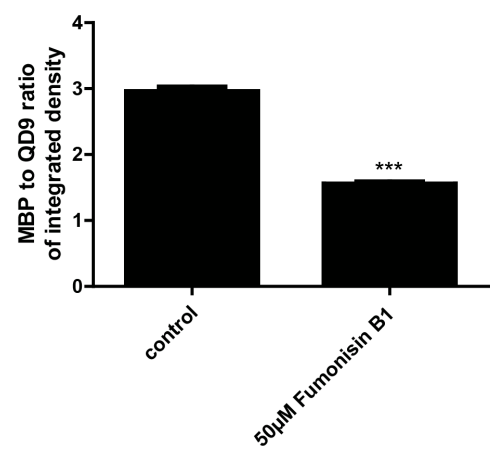

D

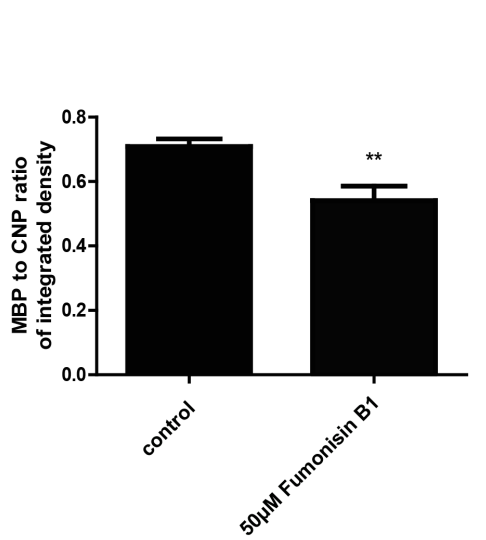

Figure 3.19: Fumonisin B1 induces QD9 unmasking and decompaction in primary mouse oligodendrocytes

Fumonisin B1 (50 $\mu \mathrm{M}$ for $96 \mathrm{~h})$ treated primary mouse oligodendrocytes were stained with MBP (in green) and QD9 (in red, A) or CNP (in red, B) (scale bar $25 \mu \mathrm{m}$ ). The quantification of the integrated density of the MBP to QD9 (in B) or CNP (in D) ratio is shown in $B$ or D respectively. Bars show mean with SEM $(n=3,>20$ cells per condition, $* *=\mathrm{P}<0.01,{ }^{* * *}=\mathrm{P}<0.001$, Student's t-test).

Another integral membrane lipid is cholesterol that is contained in the lipid rafts and detergent-resistant membrane fractions that MBP associates with. Cholesterol can be removed from cultured cells by treatment with cyclodextrins. These cyclic oligosaccharides are capable of forming soluble complexes with cholesterol that they extract from the membrane, thereby disrupting lipid rafts [349]. As cholesterol is the most abundant lipid in myelin and cultured ODC's, the sheets are retracting upon treatment. The fraction of 
MBP protein that is still contained with in the remaining sheets is highly stained with QD9 antibody. Hardly any staining of QD9 could detected in control cells (see Figure 3.20). Thus, extraction of cholesterol with Methyl$\beta$-cyclodextrin (MCD) leads to a significant decrease in MBP to QD9 ratio compared to the control indicating an increased pool of dysfunctional MBP upon treatment.

A
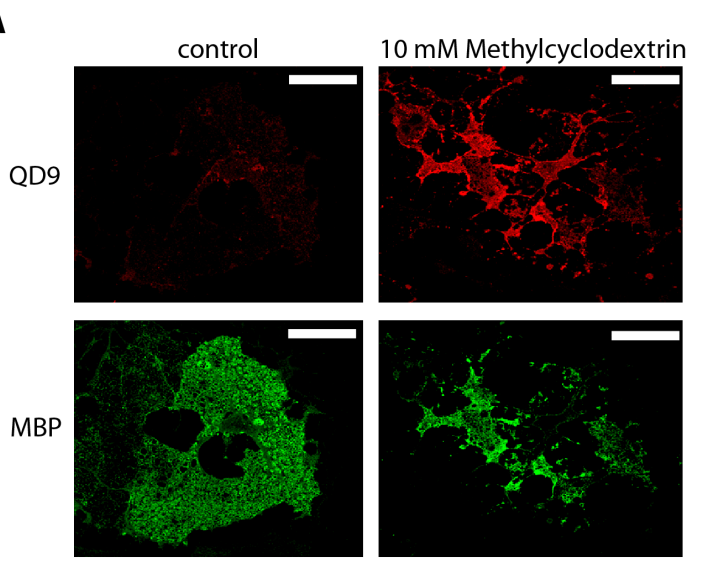

B

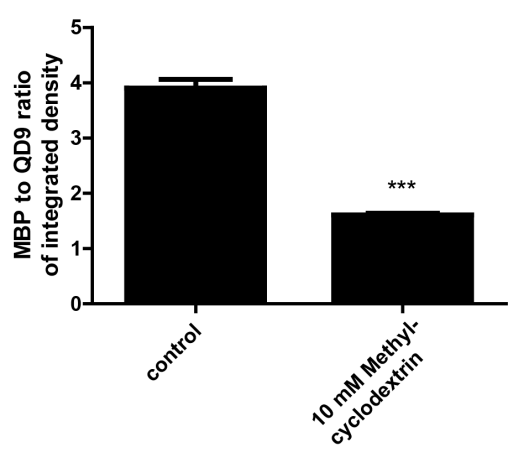

Figure 3.20: Methyl- $\beta$-cyclodextrin increases QD9 staining in the sheets of mouse oligodendrocytes

Primary mouse oligodendrocytes at DIV5 were treated with Methyl- $\beta$-cyclodextrin (10 $\mathrm{mM}$ for $5 \mathrm{mins}$ ) and then stained with MBP (in green) and QD9 (in red) (scale bar $25 \mu \mathrm{m}$ ). The quantification of the integrated density of the MBP to QD9 (in B) ratio is shown in B. Bars show mean with $\operatorname{SEM}(n=3,>20$ cells per condition, $* * *=P<0.001$, Student's t-test).

\subsection{Characterization of reverse phase transition in vitro}

As preliminary results of various treatments of cultured ODC's showed that QD9 recognized a dysfunctional state of MBP that cannot compact the membrane any longer, we studied the effect of the detachment of MBP from the membrane in more detail. Therefore, we employed antibodies detecting myelin surface epitopes, such as MOG, O1 (Galc epitope) or O4 (sulfatide epitope). Treatments of ODC's with high concentration of $\mathrm{O} 1$ antibodies led to contracted membrane sheets and redistribution of the cytoskeleton [350].

Therefore, we used these antibodies in a low concentration $(200 \mu \mathrm{M}$ for $8 \mathrm{~h})$ for treatment of mature ODC's. Treated cells were stained with a polyclonal MBP and the QD9 antibody that indicated an unmasking of the QD9 epitope in antibody treated cells. The significant decrease in MBP to QD9 
ratio was apparent for all antibodies conditions employed despite the isotype control.

In vivo the demyelinating effect of antibody treatments is often mediated via complement or serum, but our experiments in vitro showed that no serum was required to lead to a decreased MBP to QD9 ratio. This effect was already detectable after $30 \mathrm{mins}$ for $\mathrm{O} 4$ and after 60 mins for $\mathrm{O} 1$, whereas for MOG, the effect became apparent only after $4 \mathrm{~h}$ of treatment (see Figure $3.21)$. 
A
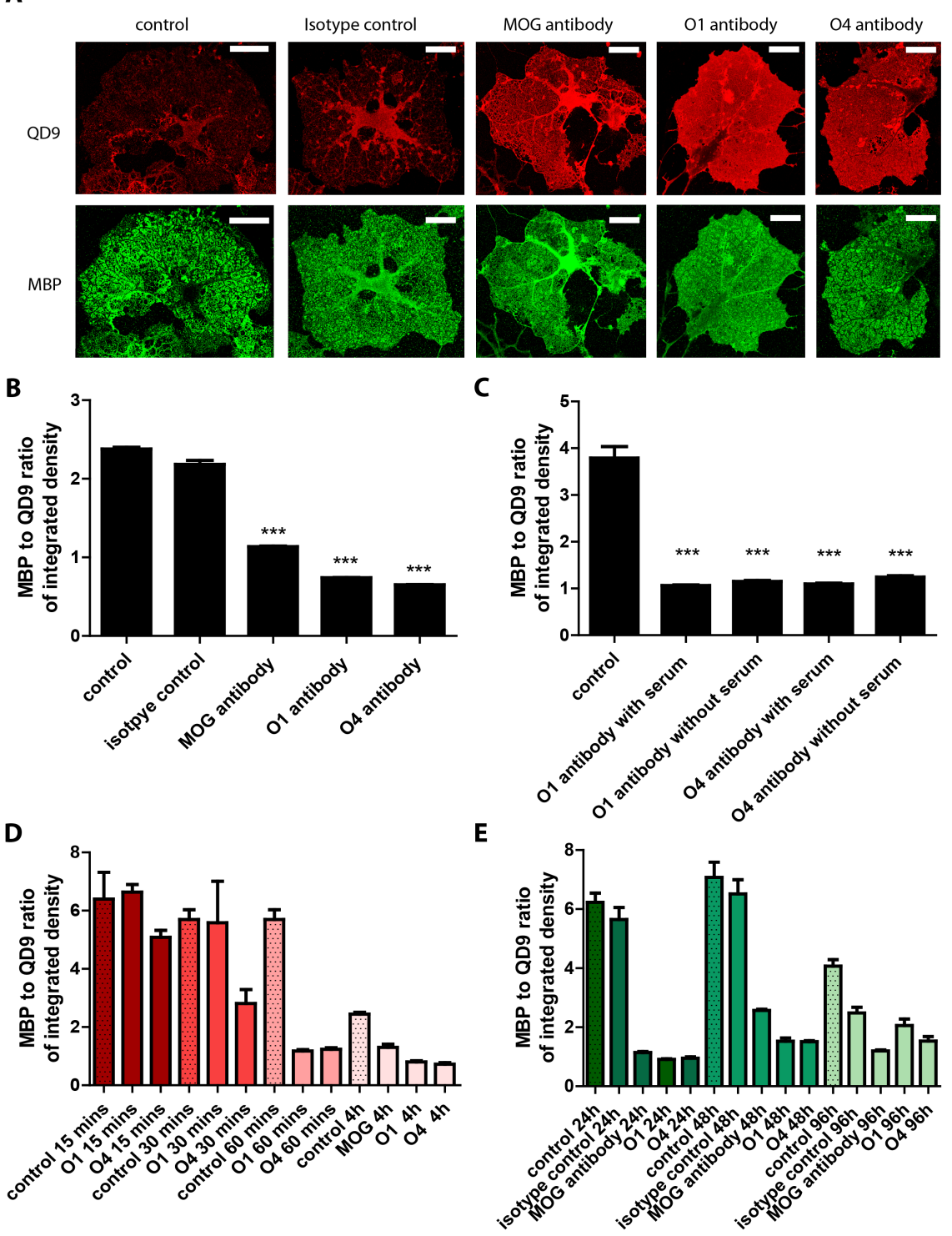

Figure 3.21: Clustering of myelin surface epitopes lead to rapid QD9 unmasking in the absence of complement

Primary mouse oligodendrocytes at DIV5 were treated with myelin surface antibodies, such as anti- MOG, O1 or O4 antibodies (200 $\mu \mathrm{M}$ for $8 \mathrm{~h}$ ) and then stained with MBP (in green) and QD9 (in red) (scale bar $25 \mu \mathrm{m}$ ). The quantification of the integrated density of the MBP to QD9 (in B) ratio is shown in B, whereas C shows the quantification for treatment in presence or absence of serum for $8 \mathrm{~h}$ treatment. D and E depict the quantification for short term and long term treatment with the antibodies respectively. Bars show mean with SEM $\left(\mathrm{n}=3,>20\right.$ cells per condition, ${ }^{* *}=\mathrm{P}<0.01, * * *=\mathrm{P}<0.001$, Student's t-test). 


\subsubsection{Detachment of MBP from the membrane upon treat- ment with antibodies against $\mathrm{O} 1$ and $\mathrm{O} 4$}

In order to further assure that QD9 stains a pool of MBP that lost its membrane binding, we stained for PIP2. As explained above, this epitope can only be stained in the sheets upon detachment of MBP from the membrane due its strong binding to MBP. Upon treatment of ODC's with myelin surface epitope antibodies against O1, O4 and MOG $(200 \mu \mathrm{M}$ for $8 \mathrm{~h})$, PIP2 staining in the sheets increased significantly indicating the detachment of MBP from the membrane (see magnification in the white box in Figure $3.22)$.

As staining for PIP2 is an indirect method of assessing the membrane binding of MBP, we wanted to support this finding using a detergent solubility assay. As MBP bind to lipid rafts under normal conditions, a high fraction of the protein is detergent insoluble. Upon detachment of MBP from the membrane, the protein becomes more detergent soluble.

For the detergent solubility assays, we treated the cells in culture as explained above and subsequently lysed them in lysis buffer containing $20 \mathrm{mM}$ CHAPS. After centrifugation of the samples, equal fractions of the pellet and the supernatant were subjected to Western blotting with an MBP antibody to assess the content of MBP in the fraction in control and treated cells. Upon treatment of ODC's with anti-O1 or O4 antibodies, the MBP signal in the pellet decreases and the supernatant increases (see Figure 3.23). It is known that MBP can be found mainly in the pellet fraction, if it is bound to lipid rafts and hence, we concluded that MBP detaches from the membrane upon clustering of lipids via anti-Galc or sulfatides antibodies. 
A

control
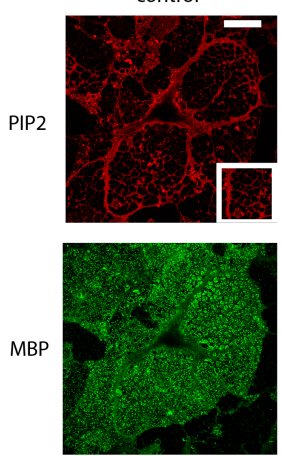

Isotype control
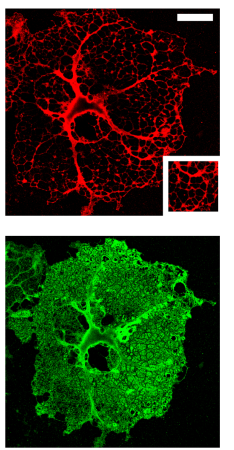

MOG antibody
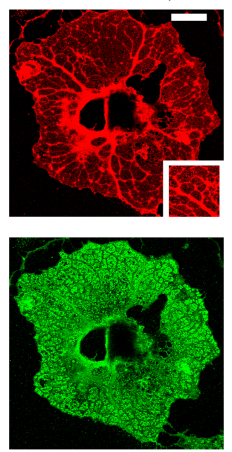

01 antibody
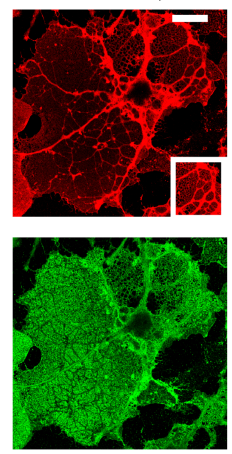

O4 antibody
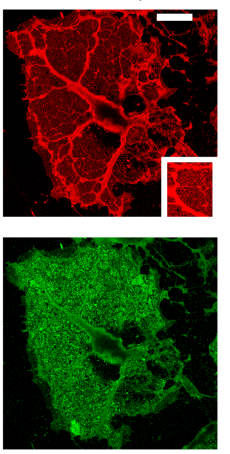

\section{B}

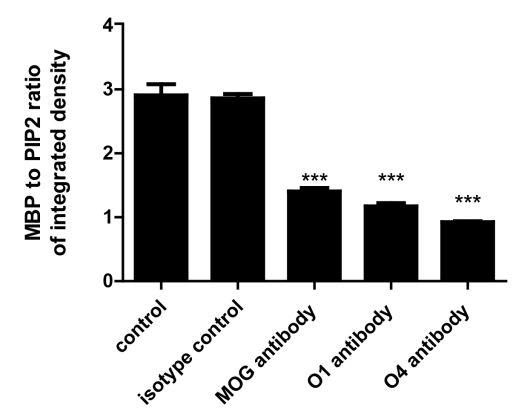

Figure 3.22: Treatment with myelin surface antibodies leads to MBP detachment from membrane

Treatment of primary mouse oligodendrocytes at DIV5 with myelin surface antibodies (O1, O4, MOG) for $8 \mathrm{~h}$ at $200 \mu \mathrm{M}$ and subsequent staining of MBP (in green) and PIP2 (in red) leads to PIP2 staining in the sheets as shown in A (scale bar $25 \mu \mathrm{m}$ ). The quantification of the integrated density of the MBP to PIP2 (in B) ratio is shown in B. Bars show mean with SEM $\left(\mathrm{n}=3,>20\right.$ cells per condition, ${ }^{* * *}=\mathrm{P}<0.001$, Student's t-test). 
A

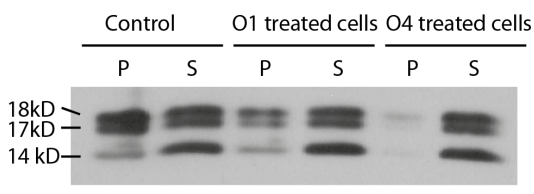

B

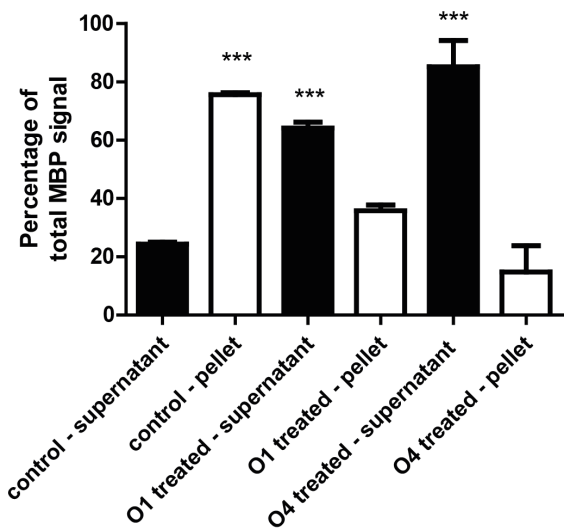

Figure 3.23: $\mathrm{O} 1$ and $\mathrm{O} 4$ treatment of cells increases the CHAPS-solubility of MBP After treatment of primary mouse oligodendrocytes at DIV5 with myelin surface antibodies (O1, O4, MOG) for $8 \mathrm{~h}$ at $200 \mu \mathrm{M}$, the cells were solubilised and the detergent-soluble fraction of the membrane with its associated proteins was extracted with CHAPS. The cell lysate was subjected to ultracentrifugation and fractions of the pellet $(\mathrm{P})$ and the supernatant $(\mathrm{S})$ were employed for Western blot analysis. A shows the result of a representative Western blot with an anti-MBP antibody, whereas B shows the quantification of the band intensities as percentage of the total MBP signal in supernatant and pellet. Bars show mean with SEM $\left(\mathrm{n}=3,{ }^{* * *}=\mathrm{P}<0.001\right.$, one-way ANOVA).

\subsubsection{Self-interaction of MBP upon $\mathrm{O} 1$ and $\mathrm{O} 4$ treatment}

So far, we could show that MBP detaches from the membrane upon treatment with $\mathrm{O} 1$ or $\mathrm{O} 4$ antibodies. As membrane binding is a prerequisite for the self-interaction of MBP, we investigated the integrity of the MBP network upon treatment with these lipid antibodies.

To determine whether MBP is self-interacting, we chose an antibody-mediated FRET assay. Therefore, we treated our ODC's with various treatments (see 2.2.2.3 for details) and afterwards fixed the cells. MBP antibodies were incubated with fluorescently labeled Fab-fragments ( $\mathrm{Cy} 3$ or $\mathrm{Cy} 5)$ and then used to stain the treated cells. Thereafter, the acceptor bleaching method was employed to study the proximity of the MBP molecules. This method relies on bleaching the acceptor leading to an increase in emission of the donor, if the molecules are within close proximity. From the fluorescence intensities before and after the bleaching of the acceptor, the FRET efficiency can be calculated and serves as a measure for the proximity of molecules.

In control cells, where the MBP network is intact and the MBP molecules are in close interaction leading to a FRET efficiency of about $20 \%$. Treatment affecting the membrane binding of MBP, e.g anti-O1 and $\mathrm{O} 4$ antibodies, as well as Ionomycin and MCD, the FRET efficiency decreased significantly to 
around $5 \%$ (see Figure 3.24). As the FRET efficiency is a measure for proximity, this dramatic reduction suggests the loss of self-interaction between the MBP molecules. The self-interaction of MBP is crucial for the network formation and its extrusion function and therefore, we next investigated the functional consequences of the loss of self-assembly upon O1 and O4 treatment. 


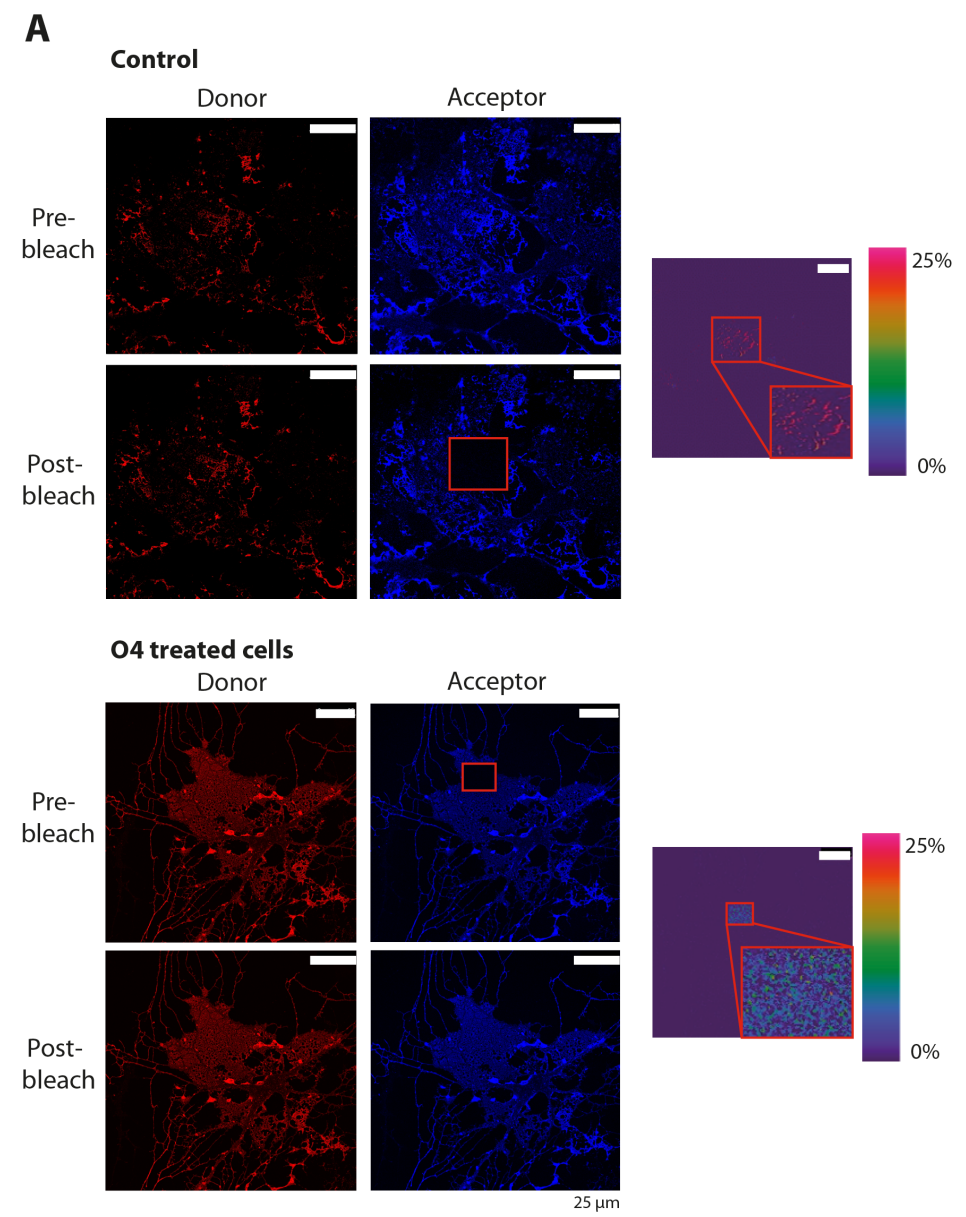

B

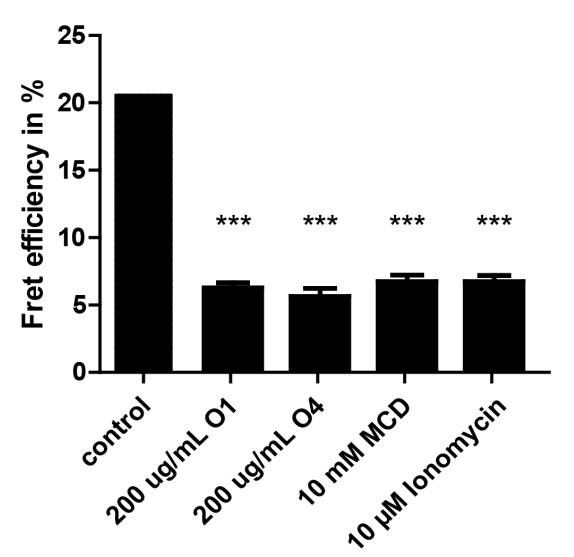

Figure 3.24: Antibody-mediated FRET assay indicates loss of self-interaction upon detachment of MBP from the membrane

Primary mouse oligodendrocytes were treated with O1 or O4 antibody ( $200 \mu \mathrm{M}$ for $8 \mathrm{~h}$ ), $10 \mathrm{mM} \beta$-methylcyclodextrin (MCD) (for $15 \mathrm{mins}$ ) or $10 \mu \mathrm{M}$ Ionomycin (for $2 \mathrm{mins}$ ) before fixation. Afterwards, the cells were stained with antibodies against MBP pre-labeled with Fab-Cy3 or Fab-Cy5. Using the acceptor bleaching FRET assay, we determined the increase in donor fluorescence after acceptor bleaching (see A) (scale bar $25 \mu \mathrm{m}$ ). From that the FRET efficiency can be calculated and that is a measure for the interaction of MBP molecules. As shown in B, the FRET efficiency decreased upon treatment with the antibodies, MCD or Ionomycin. Bars show mean with SEM $\left(\mathrm{n}=3,{ }^{* * *}=\mathrm{P}<0.001\right.$, one-way ANOVA). 


\subsubsection{Compaction and extrusion of MBP upon $\mathrm{O} 1$ and $\mathrm{O} 4$ treatment or primary oligodendrocytes}

We could show that treatment of primary mouse ODC's with O1 and O4 antibodies leads to the detachment of MBP from the membrane and a loss of self-assembly. As the self-interaction of MBP is crucial for the function of the protein, we assessed its ability to extrude proteins with spacious cytosolic domains, such as CNP from compacted areas. Hence, we treated the cells with the surface lipid antibodies $(200 \mu \mathrm{M}$ of $\mathrm{O} 1$ and $\mathrm{O} 4$ for $8 \mathrm{~h})$ and afterwards stained for MBP and CNP. In untreated or isotype control treated cells, there is a separation between CNP-positive processes and MBP-positive sheets. Only upon treatment with Galc and sulfatide antibodies, CNP can be detected in the MBP positive sheets (see Figure 3.25). This results supports our hypothesis that surface lipid antibody exposure leads not only to loss of membrane binding and self-interaction, but also to a loss of function of the MBP protein. This dysfunctional state of MBP can be selectively stained with the QD9 antibody and is a proof of principle for our hypothesis that MBP can undergo reverse phase transition. To assess, if the QD9 antibody can also be used in vivo to discriminate a pool of dysfunctional MBP, we performed immunohistochemistry on demyelinating animals models. 


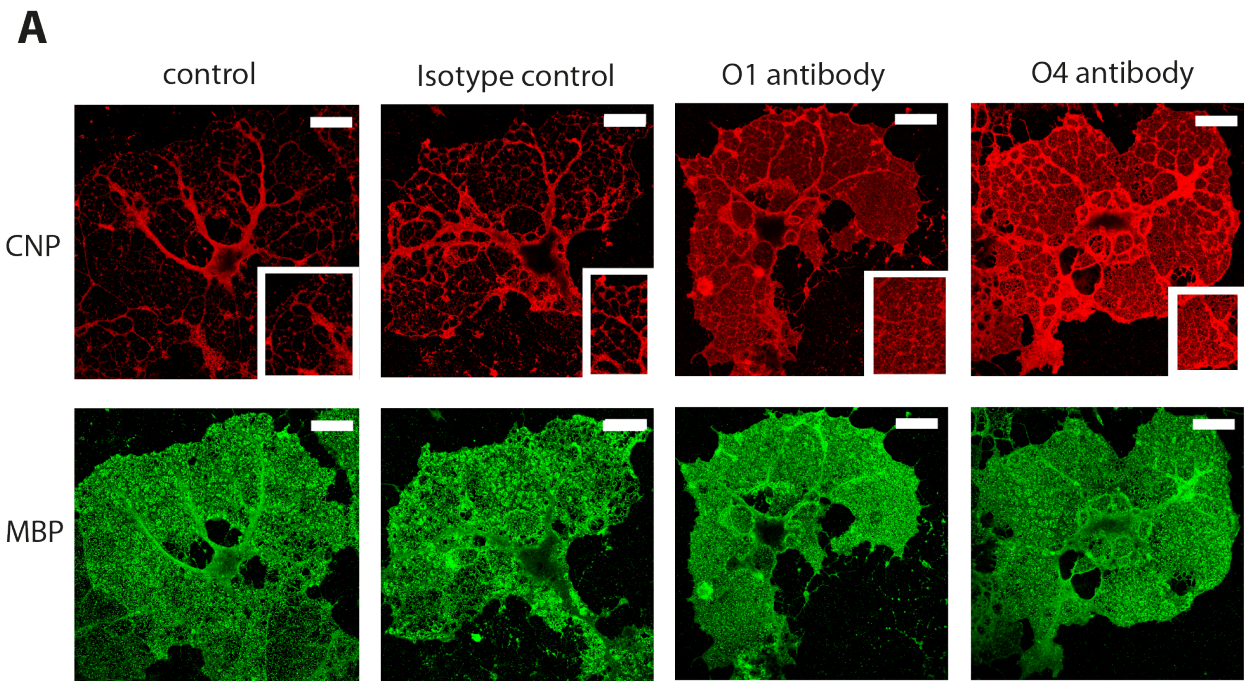

\section{B}

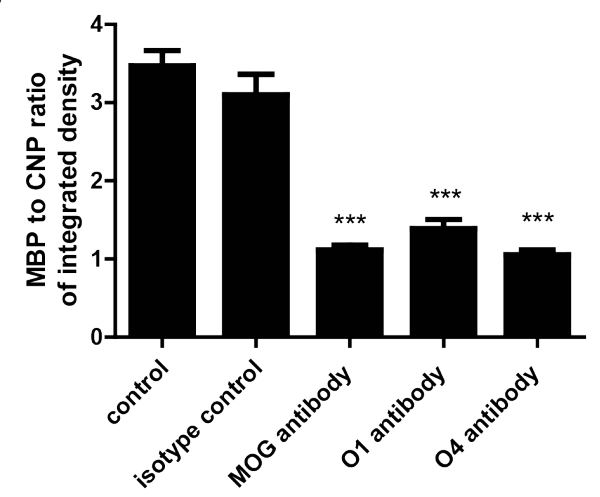

Figure 3.25: Decompaction of primary mouse oligodendrocytes upon myelin surface antibody treatment

Primary mouse oligodendrocytes treated with MOG, O1 or O4 antibody (200 $\mu \mathrm{M}$ for $8 \mathrm{~h}$ ) were stained for MBP (in green) and CNP (in red). The boxes display the usually compacted area of the cells that is devoid of CNP in control cells, but contains CNP in treated cells (A) (scale bar $25 \mu \mathrm{m}$ ). Quantification of the integrated density ratio of MBP to CNP are displayed in B. Bars show mean with SEM $\left(\mathrm{n}=3,{ }^{* * *}=\mathrm{P}<0.001\right.$, one-way ANOVA).

\subsection{Characterization of reverse phase transition of MBP in the cuprizone model in vivo}

\subsubsection{QD9 immunohistochemistry of cuprizone time course}

In culture, we could show MBP undergoes reverse phase transition into a dispersed, dysfunctional state that displays the QD9 epitope. Next, we aimed 
to assess whether this dysfunctional MBP state also occurs in vivo in early stages of demyelinating disease. Hence, we employed the widely used cuprizone model: C57B6 mice are fed with $0.2 \%$ cuprizone that induces highly reproducible demyelination after 4 weeks of treatment. To understand, if the MBP structure was altered, we performed QD9 staining on sections from mice after 1, 2, 3, 4 and 5 weeks of cuprizone exposure. Initial testing of the antibody revealed that it only works in cryo- and not in paraffin sections, if the antigen retrieval is omitted (see Method section for more details). Immunohistochemistry using the QD9 and the MBP antibody (see Figure 3.26) showed that the MBP absolute staining intensity is comparable during the whole time course apart from the 5 weeks cuprizone exposure. Using the standard monochromatic myelin stain Luxol Fast Blue, this time point displayed complete demyelination (see Figure 5.6 in Appendix). In contrast to that, the QD9 absolute staining intensity increased after already two weeks of cuprizone treatment in focal areas of the corpus callosum and peaked at 4 weeks of exposure. The QD9 intensity also declined at the 5 weeks cuprizone time point. As reported previously, there are regional differences upon cuprizone exposure as the caudal corpus callosum was shown to demyelinate before the rostral part [264]. This finding could be reproduced by our results as the QD9 intensity increased in the caudal part before the rostral corpus callosum (see Figure 3.26). Furthermore, demyelination as indicated by Luxol Fast Blue started also in the caudal corpus callosum before progression to the more frontal regions (see Figure 5.6 in Appendix). By calculating the ratio between the MBP and QD9 staining intensities, we obtained a robust measure of the dysfunctional MBP normalized to the total MBP. This ratio further indicated that dysfunctional MBP appeared already after 2 weeks of cuprizone treatment. Hence, dysfunctional MBP appears before demyelination can be detected with standard staining techniques, such as Luxol Fast Blue. 
A
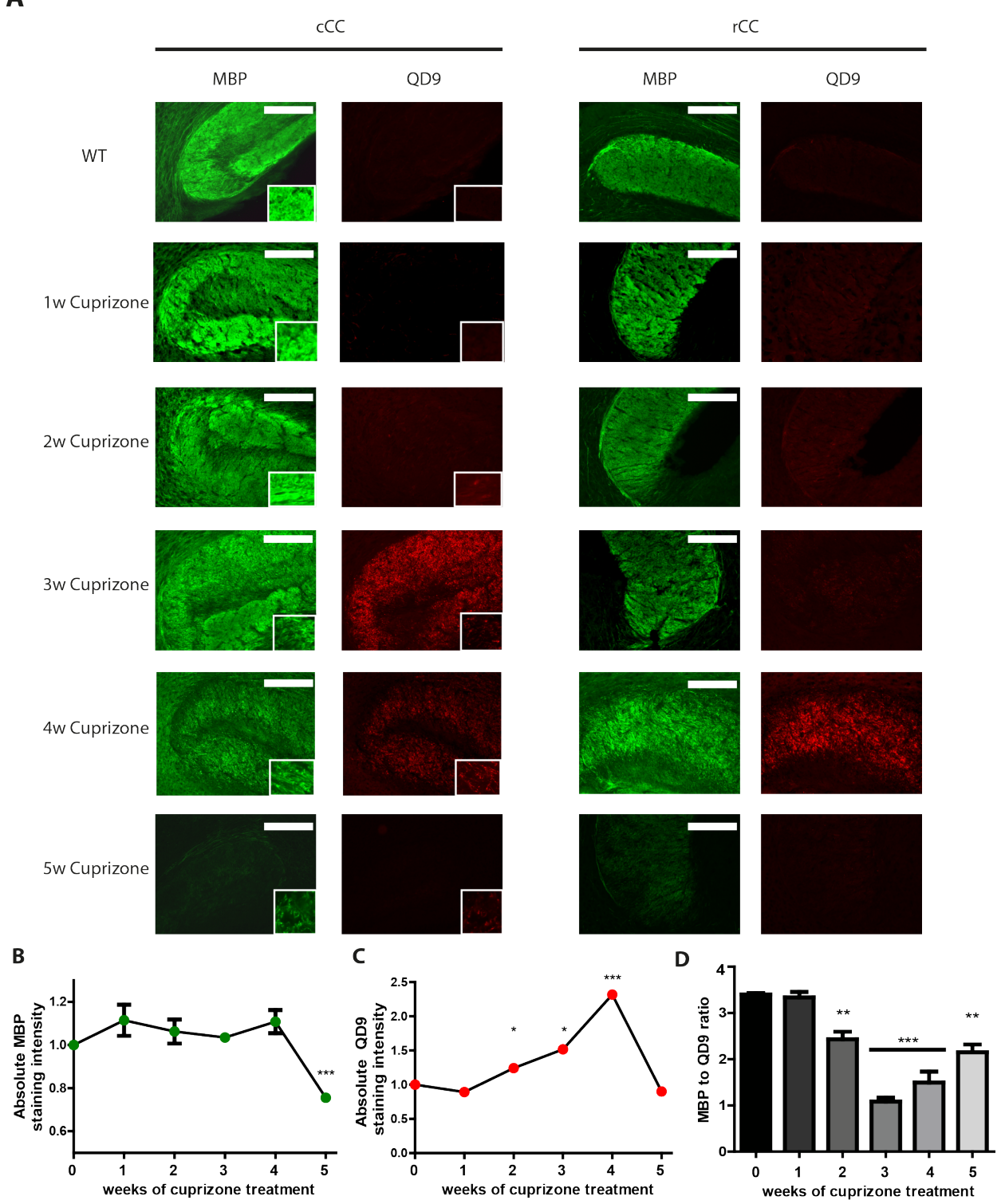

Figure 3.26: QD9 unmasking in early stages of cuprizone time course

C57B6 mice were treated with $0.2 \%$ of cuprizone and each week, one set of mice was processed for immunohistochemistry with QD9 (in red) and MBP (in green) (A, magnification of caudal corpus callosum in B) (scale bar $1 \mathrm{~mm}$ in A and $200 \mu \mathrm{m}$ in B). C and D show the absolute staining intensity of MBP and QD9 (normalized to WT) in corpus callosum respectively, whereas $\mathrm{E}$ shows their ratio over the cuprizone treatment time course. Bars show mean with SEM $\left(\mathrm{n}=3,{ }^{*}=\mathrm{P}<0.05,{ }^{* *}=\mathrm{P}<0.01,{ }^{* * *}=\mathrm{P}<0.001\right.$, one-way ANOVA). 


\subsubsection{Early loss of MAG staining in cuprizone treated mice}

Next we assessed the staining of MAG, a protein of the adaxonal space that is frequently absent in lesions of patients with demyelinating diseases and as we showed also disappears from the early lesions of NMO injections. Immunohistochemistry using MAG and MBP (see Figure 3.27) revealed that MAG was lost in caudal regions of the corpus callosum after 3 weeks cuprizone treatment. The MAG staining intensity further declined after 4 weeks of cuprizone exposure.

a
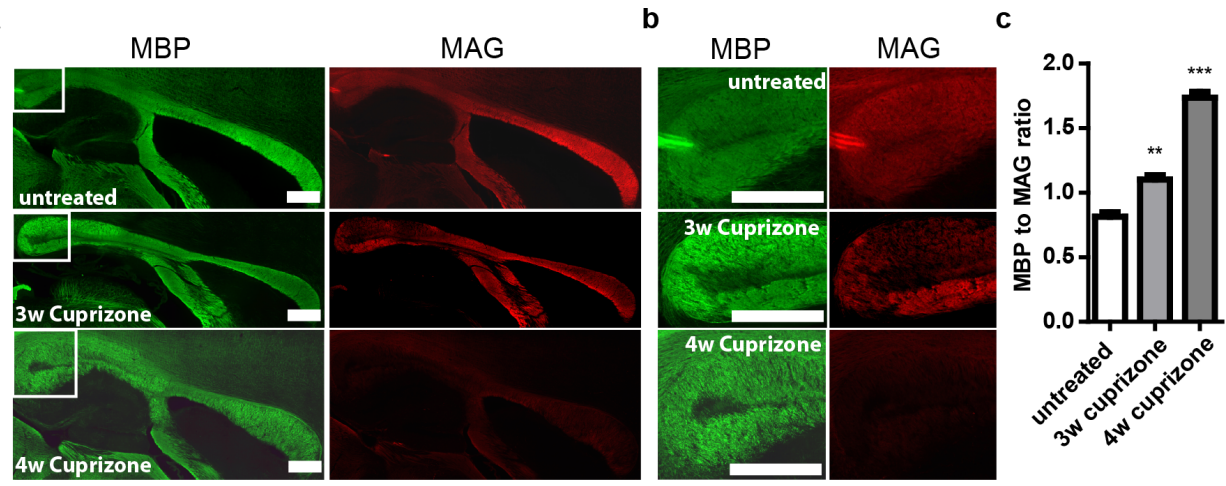

Figure 3.27: Loss of MAG staining upon short cuprizone exposure

Tissue sections of cuprizone treated mice at the indicated times were stained with MBP (in green) and MAG (in red) (A). Quantification of the signal intensities show MAG loss already three weeks after cuprizone treatment. Bars show mean with SEM $(n=3$, $* *=\mathrm{P}<0.01, * * *=\mathrm{P}<0.001$, one-way ANOVA).

\subsubsection{Myelin pathology in cuprizone time course}

The loss of MAG staining in the focal NMO model was manifested on an ultrastructural level by vesiculation of the innermost myelin lamellae. In order to establish whether this vesiculation also occurs in the cuprizone model and which myelin fragmentation patterns are stained with the QD9 antibody, we processed animals of the whole cuprizone time course (1 to 5 weeks) by high-pressure freezing for EM.

The first detectable changes were observed after 2 weeks of cuprizone exposure, where about $30 \%$ of split myelin lamellae were detected (see Figure 3.28). As shown in the white box in Figure $3.28 \mathrm{~A}$, the splitting occured not only at the intraperiodal line as published previously [268] [272], but also in the major dense line formed by MBP. After 3 weeks of cuprizone treatment, myelin pathology included split myelin sheath to about $25 \%$ of total myelin sheath, vesiculation of myelin with about $30 \%$ and degenerated myelin stacks of about $30 \%$. Further exposure to cuprizone up to 4 weeks led to an vastly increased proportion of degenerated myelin sheath up to 
$60 \%$ of all myelin sheaths assessed. Usually, the degenerated myelin displayed dramatically reduced contrast in EM and was not in association with axons any longer. Strikingly, at 5 weeks of cuprizone treatment, almost all axons were demyelinated and hardly any signs of myelin pathology was detected. Hence, the microglia and macrophages cleared the myelin debris efficiently until this then. Moreover, signs of early remyelination could be detected (data not shown). Having these ultrastructural changes upon cuprizone exposure in hand, we could correlate the QD9 staining with the myelin pathology detected. Of note, we assessed the QD9 antibody also in cryoimmunoelectron microscopy. Due to the processing of the samples and the subsequent handling, an high immunoreactivity of QD9 to MBP in control sections was detected, but was not specific to dysfunctional MBP states. Due to this finding, we can only correlate the EM data with the histology of QD9: Conclusively, we can assume that QD9 antibody detects split compact myelin, as well as areas where vesiculated myelin is present. As cryo-immunoelectron microscopy detected that the vesicules of myelin are devoid of MBP, the QD9 antibody possibly detects the pool of MBP that is currently undergoing a structural change and is not yet degraded. Hence, the cuprizone model seems to be a proof of principle for MBP undergoing reverse phase transition in vivo. 
A
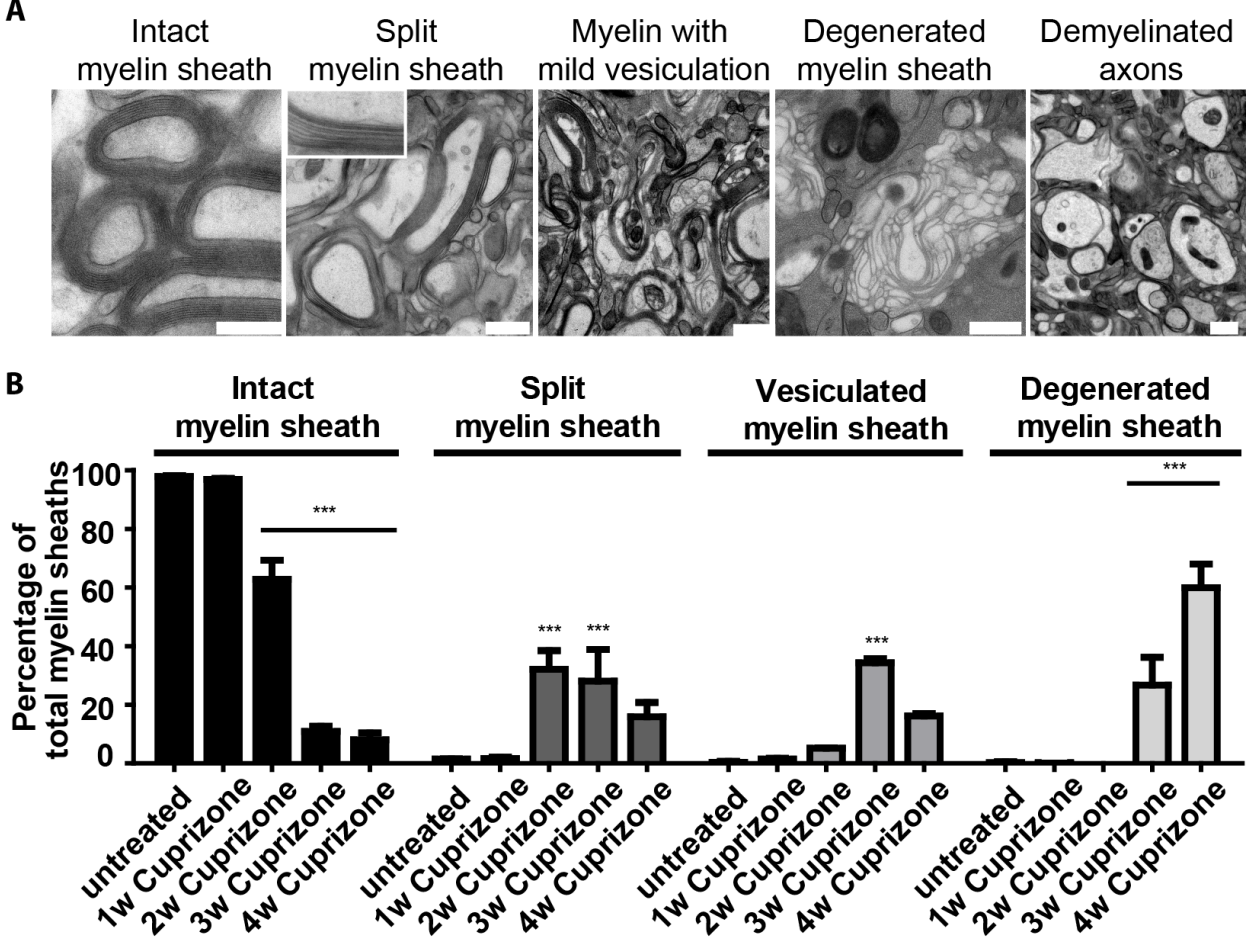

Figure 3.28: Ultrastructural changes in myelin upon cuprizone treatment Corpus callosum of C57B6N mice trated with $0.2 \%$ of cuprizone for one to five weeks were high pressure frozen for electron microscopy. A depicts representative images obtained at the different cuprizone treatment times (scale bar $500 \mathrm{~nm}$ ). The quantification of the different myelin fragmentation profiles found over time as percentage of the total myelin sheath is shown in B: split myelin sheath, vesiculated and degenerated myelin (for representative images see 2, 3 and 4 weeks cuprizone exposure in A). Bars show mean with SEM $\left(\mathrm{n}=3,3\right.$ animals per time point, ${ }^{* * *}=\mathrm{P}<0.001$, one-way ANOVA).

\subsubsection{QD9 histology in early NMO lesions}

So far, we could establish a correlation between myelin pathology and QD9 staining in the cuprizone time course. To assure this correlation with another demyelinating model, we re-assessed the focal NMO model. As decribed earlier, we could show earlier that a large fraction of myelin sheaths were vesiculated at the innermost layers at $18 \mathrm{~h}$ p.i.. We employed this time point together with $1 \mathrm{~h}$ p.i. of the AQP4 antibody and the PBS injection on the contralateral site for immunohistochemistry of QD9 and MBP (see Figure 3.29). QD9 staining of the earlier time point only showed staining at the rim of the injection site that is thought to be due to tissue damage. Upon $18 \mathrm{~h}$ p.i. PBS injection, a slight increase in QD9 staining can be detected around the lesion. For $18 \mathrm{~h}$ p.i. of AQP4 antibody, a significant increase in QD9 staining efficiency, as well as area can be detected. The MBP staining did not 
display significant variations in staining intensity. The MBP to QD9 ratio was calculated and due to the different background in the various animals assessed, we normalized the ratio at the focal NMO lesion site to the one generated with PBS. The resulting normalized MBP to QD9 ratio displayed an increase of about to twofold of the $18 \mathrm{~h}$ p.i. AQP4 lesion compared to the $1 \mathrm{~h}$ p.i. NMO lesion (see Figure $3.29 \mathrm{C}$ ).

With this focal NMO model, we could also establish a correlation between vesiculation of myelin as a result of the AQP4 antibody injection and an increase in the QD9 immunohistochemistry.

A
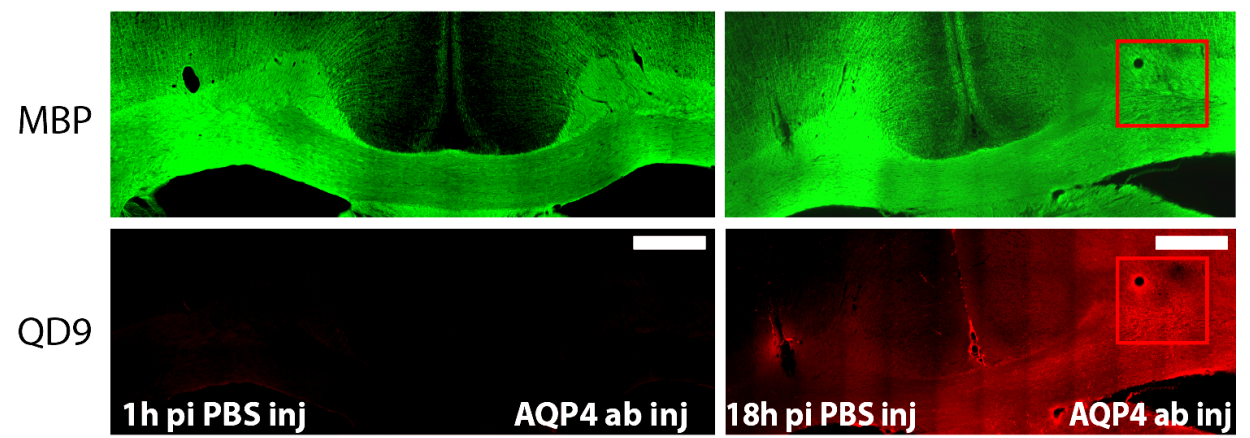

B
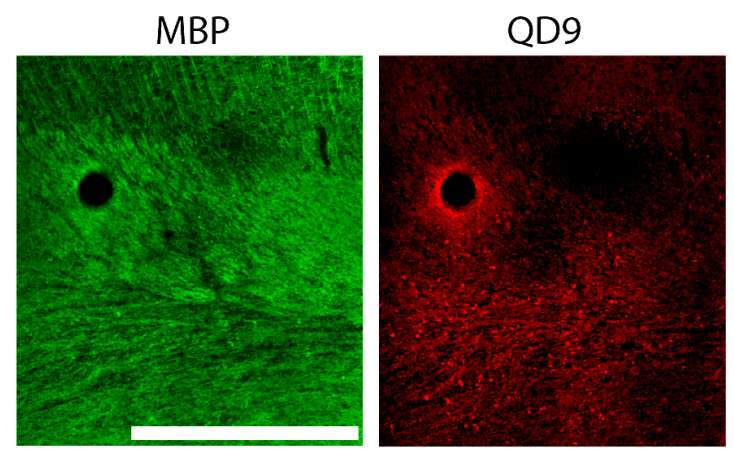

C
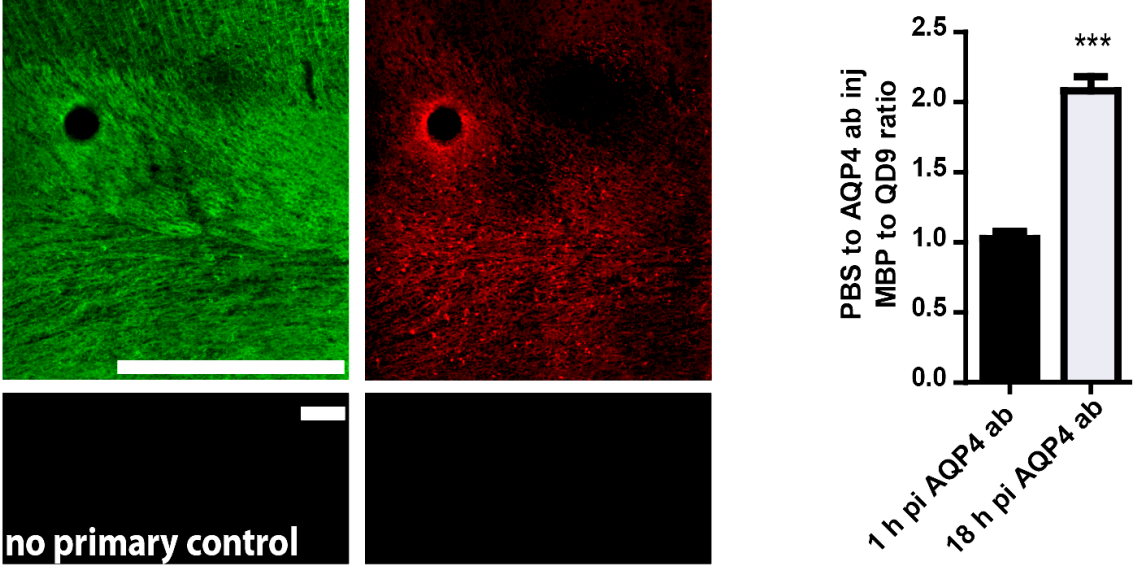

Figure 3.29: Early NMO lesions in Lewis rats display QD9 unmasking

Lewis rats were injected with human AQP4 antibody and complement and processed for immunohistochemistry after 1 and $18 \mathrm{~h}$ p.i. . The tissue sections were stained with MBP (in green) and QD9 (in red) as shown in A and a magnified version of the red box for $\mathrm{B}$ (scale bar $1 \mathrm{~mm}$ ). The quantification of the MBP to QD9 ratio is depicted in C. Bars show mean with SEM ( $\mathrm{n}=3,3$ animals per time point, ${ }^{* * *}=\mathrm{P}<0.001$, Student's t-test). 


\subsection{QD9 staining in lesions of Multiple sclerosis patients}

Finally, we investigated the QD9 and MBP staining in human brain tissue of four MS patients, as well as two control tissues in collaboration with Lukas Enz and Prof. Nicole Schaeren-Wiemers at the University Hospital Basel. Histology of healthy human control tissue did not display any staining with QD9 or only very weak staining with no clear myelin sheath morphology. In contrast to that, staining with the QD9 antibody could be detected around chronic white matter lesions of MS patients, albeit there is no or hardly any staining in the grey matter and the chronic lesion itself (see Figure 3.30). The QD9 staining can be detected in the border zone of the lesion in the cytoplasm of ODC's (see Figure 3.30) adjacent to CD68-positive cells indicative of ongoing inflammation. Hence, these findings support our hypothesis of QD9 labeling dysfunctional state of MBP that can be assumed to reside in these areas around demyelinating lesions.

A

A $\quad$ MBP

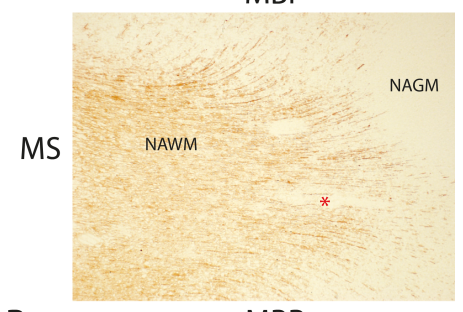

B

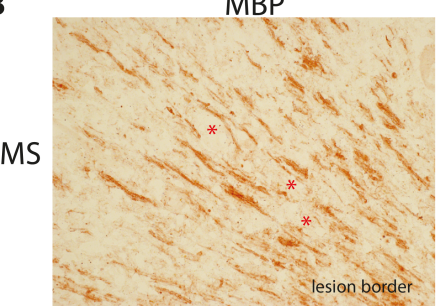

QD9

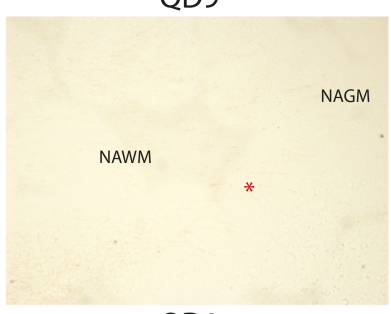

QD9

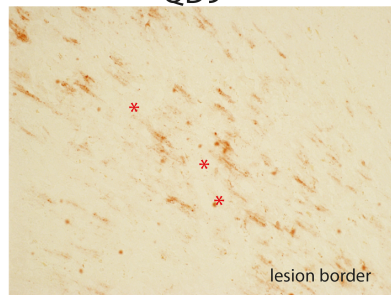

FLB

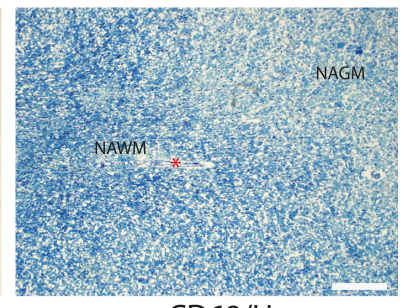

CD68/H

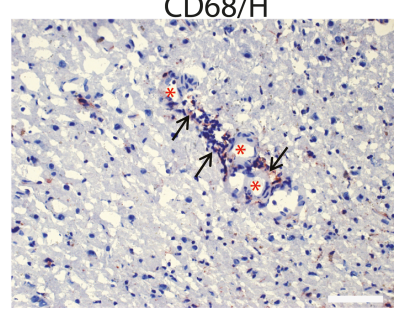

Figure 3.30: QD9 stains border zone around chronic white matter lesions of Multiple Sclerosis patients

Representative image of myelin stains at the interface from subcortical white to cortical grey matter in Multiple Sclerosis patients stained with MBP, QD9 and Luxol Fast Blue (FLB) in A and and CD68 counterstained with hemalaun (H) in B (left to right panel). (A) QD9 antibody does not stain MBP-positive fibers in normal apearing white matter (NAWM). (B) Magnification of the lesion border of the chronic active white matter lesion of the same MS case in an adjacent gyrus. QD9-positive fibers can be found in the vicinity of active ongoing inflammation as indicated by CD68-positive cells. Blood vessels are indicated by asterisks. Scale bar $500 \mu \mathrm{m}$ in A, $100 \mu \mathrm{m}$ in B. Abbreviations: FLB, fast luxol blue; NAWM, normal appearing white matter; NAGM, normal appearing grey matter. Staining of human samples was carried out in collaboration with Lukas Enz and Prof. Nicole Schaeren-Wiemers at the University Hospital Basel. 


\subsection{Calcium as underlying cause for reverse phase transition of MBP}

So far, we could show that MBP undergoes reverse phase transition to a dysfunctional state in early stages of demyelinating conditions in vivo. The dysfunctional MBP is associated with the loss of compaction in vivo as indicated by the vesiculation detected in the various animal models. Having established the tools to detect different pools of MBP molecules in vivo, we assessed whether we could induce reverse phase transition of MBP and hence, trigger myelin fragmentation.

\subsubsection{Treatment of acute brain slices with Ionomycin}

I have shown that increased intracellular calcium leads to MBP detachment from the membrane and our laboratory has further shown that treatment of brain slices with Ionomycin leads to vesiculation of myelin stacks [348].

Therefore, we treated acute brain slices with $50 \mu \mathrm{M}$ Ionomycin and performed immunohistochemistry with QD9 and MBP, but also EM on highpressure frozen samples after different treatment times from 5 to 30 mins. Histology of the Ionomycin treated brain slices revealed an unmasking of QD9 in early time points and a significant decrease in MBP to QD9 ratio was detected upon 15 and 30 mins of Ionomycin exposure (see Figure $3.31 \mathrm{~A}$ and B). At the same time, the EM of high-pressure frozen samples indicates a vesicular disruption after 15 mins in about $40 \%$ of myelin sheaths assessed. Prolonged treatment with Ionomycin up to $30 \mathrm{mins}$, further increased the vesiculation of the inner myelin layers to about $60 \%$. This vesiculation of myelin was indistinguishable from myelin fragmentation observed in focal NMO lesions. 
A
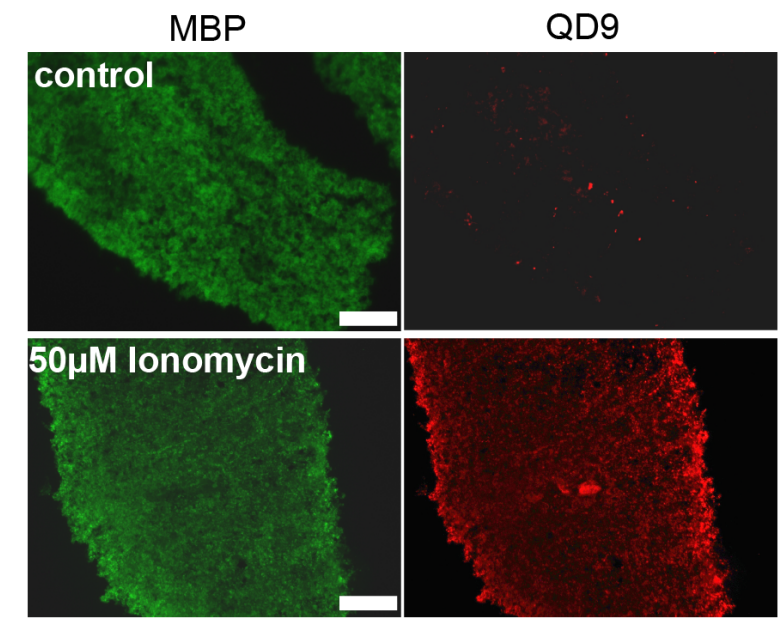

C

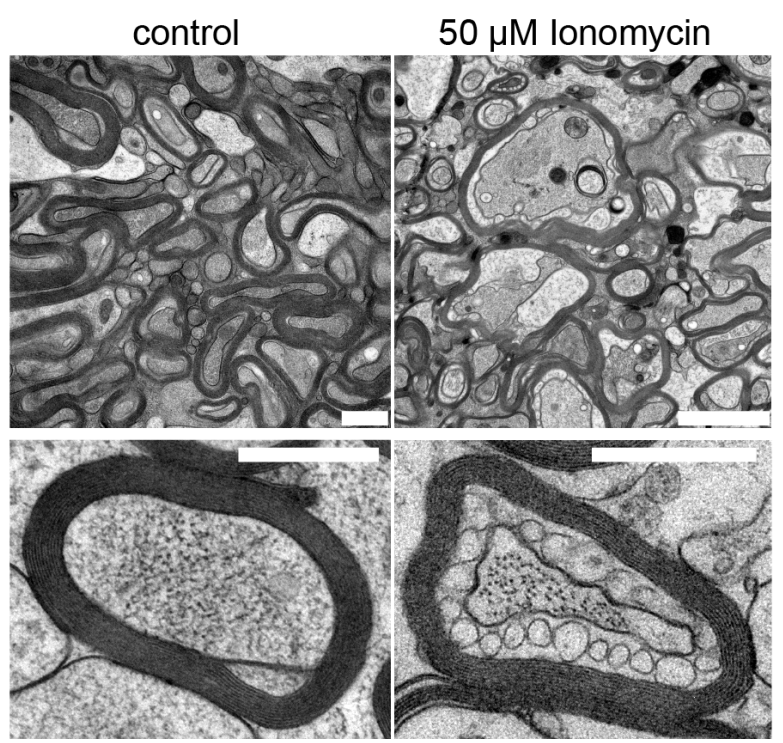

B

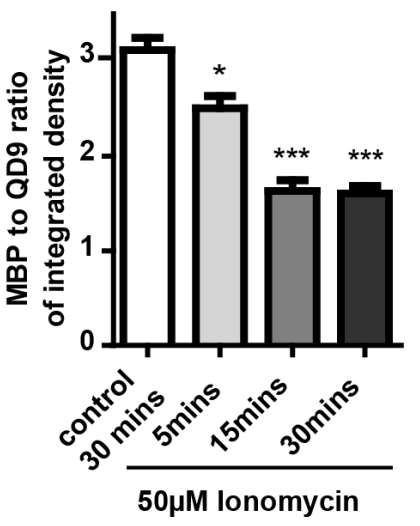

D

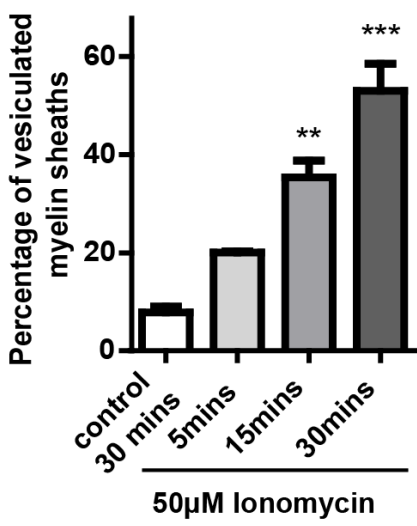

Figure 3.31: Vesiculation of the innermost myelin sheath upon Ionomycin treatment of acute brain slices

Acute brain slices were obtained from adult mice and treated with $50 \mu \mathrm{M}$ Ionomycin in aCSF and subsequently processed for immunohistochemistry or high-pressure frozen for electron microscopy analysis. The tissue was stained with MBP (in green) and QD9 (in red) (A) (scale bar $200 \mu \mathrm{m}$ ) and the signal was quantified in B over a short treatment time course. The representative images of electron micrographs are depicted in $\mathrm{C}$ (scale bar $500 \mathrm{~nm}$ ) and the percentage of vesiculated myelin sheath over the Ionomycin treatment time course was quantified in D. Bars show mean with SEM ( $\mathrm{n}=3,4$ section of different animals per time point, ${ }^{*}=\mathrm{P}<0.05,{ }^{* *}=\mathrm{P}<0.01,{ }^{* * *}=\mathrm{P}<0.001$, one-way ANOVA). 


\subsubsection{Treatment of ex vivo brain slices with EGTA after AQP4 antibody injection in Lewis rats}

My studies could show that AQP4 antibody injection in Lewis rats causes myelin vesiculation and QD9 unmasking at early time points. In order to shown that this process is mediated by calcium influx, acute brain slices of early NMO lesions (12 h p.i.) were prepared. Subsequently, ex vivo treatment of the lesions with $25 \mathrm{mM}$ EGTA or only artificial cerebrospinal fluid (aCSF) was carried out for $2 \mathrm{~h}$ at RT. Afterwards, the staining of the acute slices with GFAP revealed the GFAP loss of $30 \%$ in the NMO lesions after $12 \mathrm{~h}$ p.i. despite of the subsequent ex vivo treatment. Furthermore, the lesion area was determined by loss and fragmented astrocytes as displayed in Figure 3.32 and was increased significantly in all conditions compared to the complement only injection. Moreover, I carried out staining of the acute brain section of the NMO lesions treated with EGTA ex vivo with MBP and QD9. The increase in QD9 staining was abolished by treatment with EGTA of early NMO lesions (see Figure 3.32). Hence, calcium influx seems to be mediating the QD9 unmasking in vivo.

In conclusion, by disruption of the membrane binding of MBP via increased intracellular calcium, reverse phase transition can be induced ex vivo by e.g. Ionomycin treatment. This leads to an increase in dysfunctional MBP molecules detected by QD9 histology. Hence, the QD9 staining can be serve as a histological marker to assess myelin pathology in vivo due to a strong correlation of QD9 staining and myelin fragmentation detected in EM. 
A

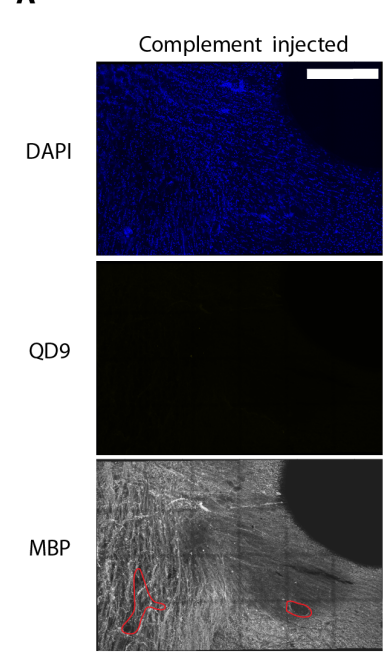

B

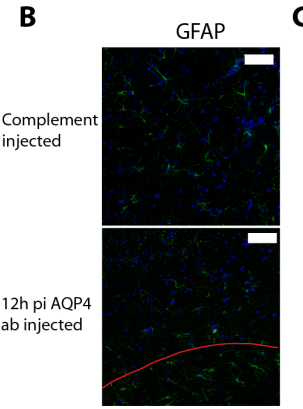

C

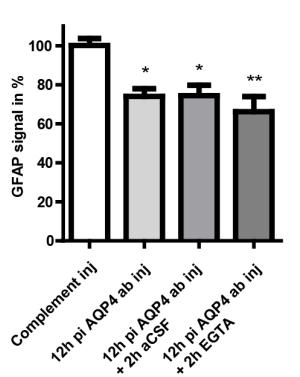

D 12h pi AQP4 ab injected

$12 \mathrm{~h}$ pi AQP4 ab injected 12h pi AQP4 ab inject
$+2 \mathrm{~h}$ aCSF
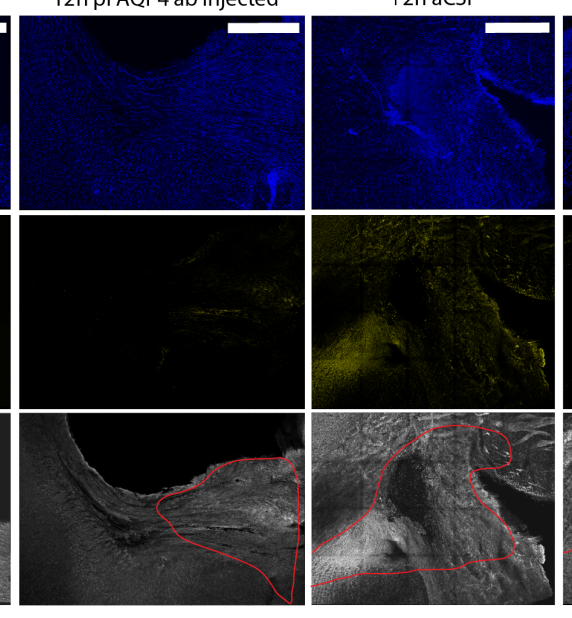

12h pi AQP4 ab injected
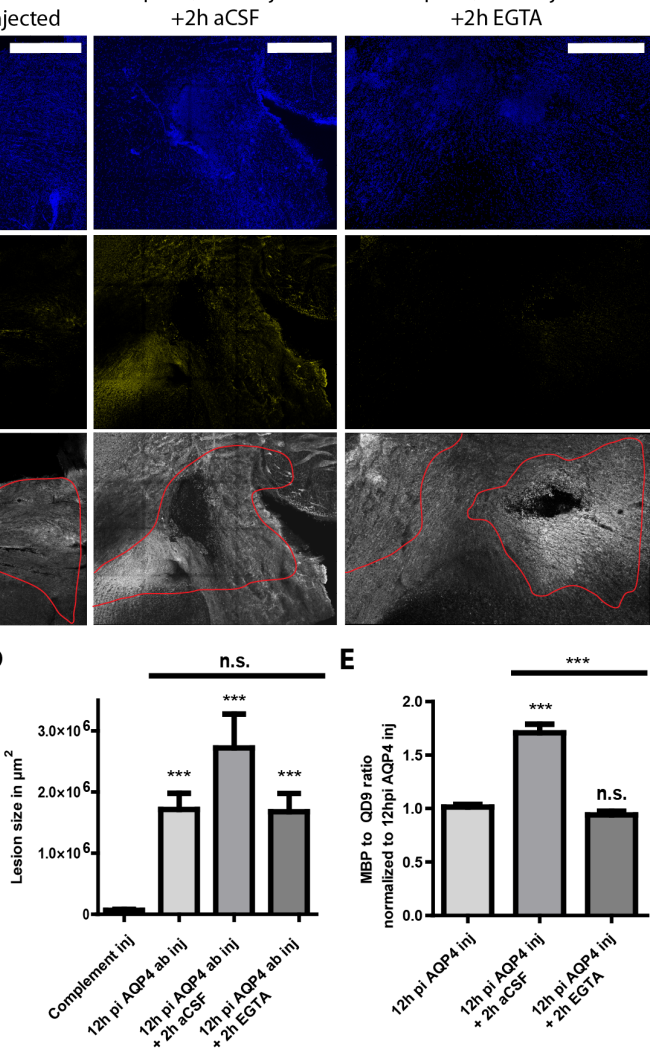

E

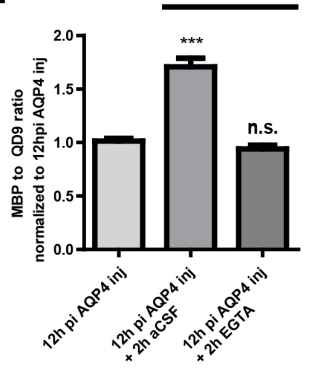

Figure 3.32: EGTA treatment of acute brain slices after AQP4 antibody injection prevents QD9 unmasking

Lewis rats were injected with AQP4 antibody PBS or PBS and human complement. After $12 \mathrm{~h}$ pi., acute brain slices were prepared and treated ex vivo with $25 \mathrm{mM}$ EGTA in aCSF or aCSF alone for $2 \mathrm{~h}$. The acute brain slices were then stained for DAPI (in blue), MBP (in grey) and QD9 (in red) (scale bar $500 \mu \mathrm{m}$ ). The lesion area marked by the red line was determined by the GFAP staining depicted with representative images in B (scale bar $75 \mu \mathrm{m}$ ). The GFAP signal in the lesion area was quantified in C, whereas D displays the size of the lesion as determined by lost and/or fragmented astrocytes. The MBP to QD9 intensity ratio that was normalized to the $12 \mathrm{~h}$ pi AQP4 antibody is displayed in E. Bars show mean with SEM $\left(\mathrm{n}=4, *=\mathrm{P}<0.05,{ }^{* *}=\mathrm{P}<0.01, * * *=\mathrm{P}<0.001\right.$, one-way ANOVA $)$. 



\title{
Chapter 4
}

\section{Discussion}

\author{
"By three methods, we may \\ learn wisdom: \\ First, by reflection, \\ which is the noblest; \\ second, by imitation, \\ which is the easiest; \\ and third, by experience, \\ which is the bitterest." \\ -Confucius
}

Myelin is a specialized membrane that spirally wraps around axons to enable saltatory nerve conduction and trophic support of axons. This multilamellar structure is extremely stable as the proteins localized to the compact myelin are among the most long-lived proteins in the body [27]. However, myelin is also the target of many autoimmune diseases of nervous system. The scope of the thesis was to shed light on the molecular mechanisms leading to the destabilization and disassembly of myelin in diseases. I aimed to investigate the myelin fragmentation at early stages of demyelinating pathologies.

The study of myelin structure in different rat and mouse models of demyelination showed that the earliest signs of myelin pathology could be mainly detected at the innermost layers of myelin. Over time, the vesiculation of the innermost lamellae progressed and involved an increasing number of previously compacted myelin lamellae. The completely fragmented myelin sheath was never found in association with axons and the demyelinated axons were found adjacent to the remaining lipid membranes.

So far, MBP is the only known protein necessary for the compaction of the cytoplasmic leaflets. We could show that upon changes to the membrane environment leading to calcium influx, MBP detaches from the membrane and undergoes a conformational change. Hence, the protein is not able to 
self-interact and compact the membrane any longer. Moreover, this dysfunctional MBP seems to allow membrane vesiculation. We propose the QD9 antibody as a tool that allows the discrimination between the nonmembrane bound, dispersed state and the membrane-bound cohesive network of MBP. With the help of the QD9 antibody, a correlation between the dysfunctional, dispersed MBP and the vesiculated state of myelin could be established. Taken together, our data suggests that the generation of dysfunctional MBP in early stages of myelin disassembly and that this is the key event for subsequent myelin fragmentation.

\subsection{Myelin pathology in demyelinating diseases}

Within the framework of these studies, I investigated several different demyelinating models. I not only studied demyelinating models in mice, but also rats: The toxin mediated models based on injection of lysolecithine or cuprizone treatment and the immune-mediated models based on the injections of the antibodies anti-AQP4 and anti-MOG.

\subsubsection{Myelin pathology in oligodendropathies}

Cuprizone is a copper chelating agents that disrupts the mitochondrial respiration leading to ODC apoptosis. Apoptotic ODC's with enlarged mitochondria were observed to appear within the first week of cuprizone exposure [271], which is in line with our results. Interestingly, the earliest signs of oligodendroglial degeneration were reported only upon two to three weeks of cuprizone treatment. The observations by Blackmore [272] [351] included splitting of the intraperiodal line, as well as the expansion of the inner tongue that was filled with fibers and vesiculated tubular material. They also reported demyelination at 5 weeks upon phagocytosis of vacuolized myelin by microglia.

We obtained similar results, despite that the splitting of the myelin sheath detected as early as 2 weeks of cuprizone exposure, did not only occur at the intraperiodal line, but also involved the major dense line. This new observation was achieved by state-of-the-art sample preparation for EM, the high pressure freezing. With the help of this technique, chemical fixation and dehydration of the sample can be avoided leading to samples that are fixed in a close to native state. This approach enabled me to detect subtle changes in myelin compaction by avoiding artefacts introduced by chemical fixation. Hence, the earliest sign of myelin pathology upon the cuprizone treatment of mice was splitting of the intraperiodal and major dense line. Three weeks of cuprizone exposure resulted in a vesiculation of the innermost myelin lamellae that was not only increasing in the extent of vesiculation (less compact 
myelin layer detected), but also affected a higher percentage of sheaths upon further exposure. Surprisingly, only one week later all fragmented sheaths were removed, to only leave the naked axons behind.

Interestingly, a comparable time course was found in an alternative model, where ODC expressing the diphtheria toxin receptor were ablated with diphtheria toxin leading to the death of these cells within one week [352]. The myelin pathology started only 3 weeks later and complete demyelination was detected after 5 weeks. In contrast to that, the demyelination was not widespread and induced only moderate microglial activation.

In the cuprizone and the diphtheria toxin model, acute axonal injury was observed prior to demyelination supporting the hypothesis that myelin provides trophic support to axons [352]. In this context, it would be crucial to understand when and how the axon senses the missing trophic support of the degenerating ODC's. So far, this question still remains to be elucidated. It can be hypothesized that the vesiculation of the inner tongue that interferes with the adhesion to the axon is one signal. Aged transgenic animals deficient of MAG exhibit signs of axonal damage [41] [196]. As this is a rather late phenotype, it is unlikely to be the only signal to the axon. Transporters and enzymes that ensure the neuronal high energy demand (such as monocarboxylate transporter 1) [353] and gap junctions that remove the excess potassium produced during axonal firing (Connexin 32 and 47; and their astrocytic outerpart Connexin 30 and 43) might also take part in mediating axonal injury [354]. More research in this area is necessary to understand the complex interplay between myelin and axons and to understand the detrimental effect of myelin loss on axons.

The toxin mediated model investigated was the stereotactic injection of lysolecithin in the corpus callosum of mice. This membrane solubilizing agent directly acts on the myelin sheath, but also leads to toxicity of myelinating cells. Upon focal injection in the brain, I could detect that $6 \mathrm{~d}$ p.i. the myelin sheatsh in the vicinity of the injection site were also split and severely vesiculated. Similar myelin pathology was detected by Fu et al. [355] after already 90 mins with $10 \mathrm{mg} / \mathrm{mL}$ lysolecithine injection. Their group could establish a clear correlation between the degradation of myelin at ultrastructural level and a decrease in coherent anti-Stokes Raman Scattering ex vivo and in vivo after lysolecithin injection. A similar correlation was established between the myelin pathology in EM and MRI with the help of the cuprizone model. These findings allow the use of these methods for in vivo time course studies of animal models of demyelinating diseases and might facilitate pharmaceutical trials.

In order to investigate, if there is a putative common mechanism between the different demyelinating models, immune-mediated models of oligodendro- 
pathy were also assessed in term of the myelin fragmentation profiles. First, I assessed the stereotactic injections of an AQP4 antibody that was derived from an NMO patient. This focal NMO model leads to apoptosis of astrocytes within a few hours p.i. due to the binding of the AQP4 antibody to the astrocytic endfeet. While the mechanism of toxicity of the AQP4 antibody is not clear, it is generally agreed that AQP4 is necessary to mediate the astrocytopathy of the AQP4 autoantibodies. Furthermore, the loss of astrocytes was found to be mediated by complement and an involvement of microglia and macrophages was shown to play a role [307]. It was suggested that astrocytes release a so far unknown soluble factor that renders ODC's vulnerable and leads to secondary demyelination. Several groups suggested a metabolic coupling of ODC and astrocytes as connexin 32 and 47 deficient mice cause ODC death. To this end, more research is necessary to investigate the cause of apoptosis of ODC after loss of astrocytes.

In line with previous reports, in our focal NMO model, a loss of astrocytes was apparent already after $12 \mathrm{~h}$ p.i. as identified by the significantly reduced GFAP and AQP4 antibody staining around the lesion site. Electron microscopy observations after $18 \mathrm{~h}$ p.i. detected about $70 \%$ of myelin sheaths with vesiculation at the inner tongue. A later time point ( $5 \mathrm{~d}$ p.i.) displayed a high degree of severe vesiculation and myelin sheath degeneration, whereas after 1 week p.i. a very heterogenous pattern of these fragmentation profiles was observed. To my knowledge, this is so far the first electron microscopic study of a demyelinating animal model for NMO. Importantly, similar fragmentation profiles as for the cuprizone time course were detected in the focal NMO. This leads to the assumption that demyelinating diseases potentially undergo myelin fragmentation in a similar way prior to demyelination.

\subsubsection{Immune-mediated myelin pathology}

In order to prove whether the sequence of myelin pathology was a common feature in many demyelinating animal models, we also assessed focal injection of MOG antibody and complement in the corpus callosum of Lewis rats. This immune-mediated model is assumed to bind to the myelin sheath directly and exert its demyelinating effect. In our electron microscopic studies, we detected split myelin sheath, an enlarged inner tongue, as well as vesiculated myelin. To my knowledge, no studies on focal MOG antibody injection alone were carried out on the ultrastructural level.

The immune-mediated EAE model in Biozzi mice we applied showed similar myelin pathology as determined for the other models by displaying an enlarged inner tongue and vesiculated myelin sheath in the adaxonal space. Moreover, we also detected vesicules within the compacted myelin layer that 
were associated with focal bulging of the whole sheath. This finding might be due to the pulling of microglia on the surface of the sheath. Despite that, the myelin fragmentation pattern in EAE were more heterogenous that in the focal NMO model as we determined other myelin pathology at a low percentage, such as myelin bulbs and microglia that seems to be attached to the myelin sheath. One possible explanation for the formation of these myelin bulbs is the pulling of microglia on the sheaths. Further research in the generation of these myelin bulbs will be necessary.

\subsubsection{Mechanism of myelin fragmentation}

Taken together, we could show that all demyelinating diseases follow a similar myelin fragmentation pattern (see Figure 4.1): Initially, the adaxonal space of the myelin sheath becomes enlarged. This is followed by vesiculation at the innermost myelin layers, which progresses until all previously compacted myelin layers are affected. This leaves behind the nude axon and the fragmented myelin sheath. In immune mediated models that target the sheath directly, the compact myelin - in addition to the fragments described- was split and contained vesicules that formed focal bulging of the sheath prior to demyelination.

These findings of a common mechanism for the early events of myelin fragmentation suggest a retrogradual spread of myelin pathology. Here, the distal processes of the ODC's are affected at the metabolically active site and progress further till the whole compact myelin is vesiculated. Subsequently, the cell body degenerates, which suggests a "dying back oligodendropathy". A similar observation was made by Ludwin and Johnson [270] when examining the cuprizone model and the "dying back" pathology of ODC's was also suggested to be involved in the formation of MS lesions [356]. The inability of the metabolically stressed ODC to support its distal processes is also revealed by the preferential loss of MAG in early stages of virus mediated models of demyelination and is also evident in MS and NMO lesions [213] [201] [294].

Overall, there seems to be a common sequence of myelin fragmentation regardless whether the insult is due to direct attack of the myelin sheath or secondary due to a toxic insult. This hypothesis is supported by the observation of vesiculated myelin sheath in various demyelinating models in the CNS and PNS: in animal models of leukodystrophies, e.g. the twitcher mouse [357]; and of the extrapontine myelinolysis of the osmotic demyelinating syndrome, further in injection of phospholipidase A2 (the enzyme that hydrolyzes PC to lysolecithin, [358]), upon treatment with calcium ionophores [359] [360], in intraneural injection of anti-Galc [361] or anti-myelin serum [362], in Theiler's virus infection [363], upon spinal cord injury [364] etc. 
These fragmentation profiles were also observed in Guillan Barre-syndrome and MS [219] [356] [365] [366] [220]. Within the lesion center, usually complete demyelination was observed, whereas at the rim of the lesions swollen and vesiculated adaxonal compartments were detected with normal appearing axons. The detection of similar myelin fragmentation profiles highlights the relevance of this study and the necessity to find agents that block myelin pathology from progressing or that quickly restore normal myelination by promoting remyelination.

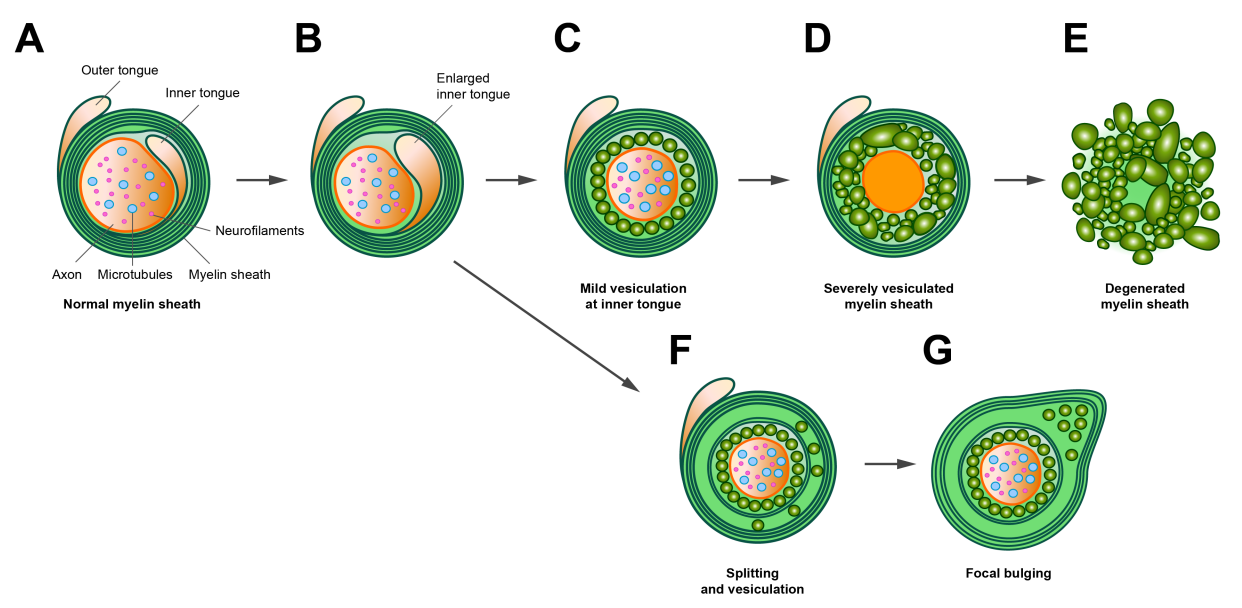

Figure 4.1: Myelin fragmentation in demyelination

Schematic drawing of myelin breakdown in early stages of demyelination. A.) cross-section of a myelinated axon (with microtubules in blue and neurofilaments in pink). Myelin membrane is highly compacted with its tight stacks of membrane (in green), but contains cytoplasm-rich domains at the outer and inner tongue (depicted in orange). Initially, the inner tongue becomes enlarged (B) before it collapses into small vesicles in the adaxonal space $(\mathrm{C})$. The vesiculation progresses outwards over time (E) before the whole myelin stack is degenerated (F). In immune-mediated model, splittings a with the membrane stacks can also contain vesiculated myelin. Focal bulging of the membrane seems to occur before myelin is removed from the axon.

The involvement of immune cells during demyelination is tremendous as it was shown that the myelin sheath can be stripped by invading macrophages [219] [220]. Similar results were detected in the EAE models, where the microglia were found in association with the paranodes, but infiltrating macrophages were physically stripping the sheaths from the axons. Hence, monocyte-derived macrophages seem to initiate the demyelination, whereas microglia clear the resulting debris [367]. During my studies, I could detect microglia or macrophages in close association to vesiculated myelin at the ultrastructural level, but also in histology. There, the microglia processes were in close contact with QD9 positive fibers and even engulfed QD9 positive myelin debris (see Figure 4.2). Hence, they seem to clear degenerating 
myelin, but an active role of these cells in demyelination would need to be further assessed. Moreover, it is an ongoing debate in the field, whether the demyelination is caused by the immune system or whether the immune response is caused by the myelin pathology. Therefore, it is of interest to investigate further when the different immune cells get involved and to assess the underlying cause to shed light on this fundamental question.

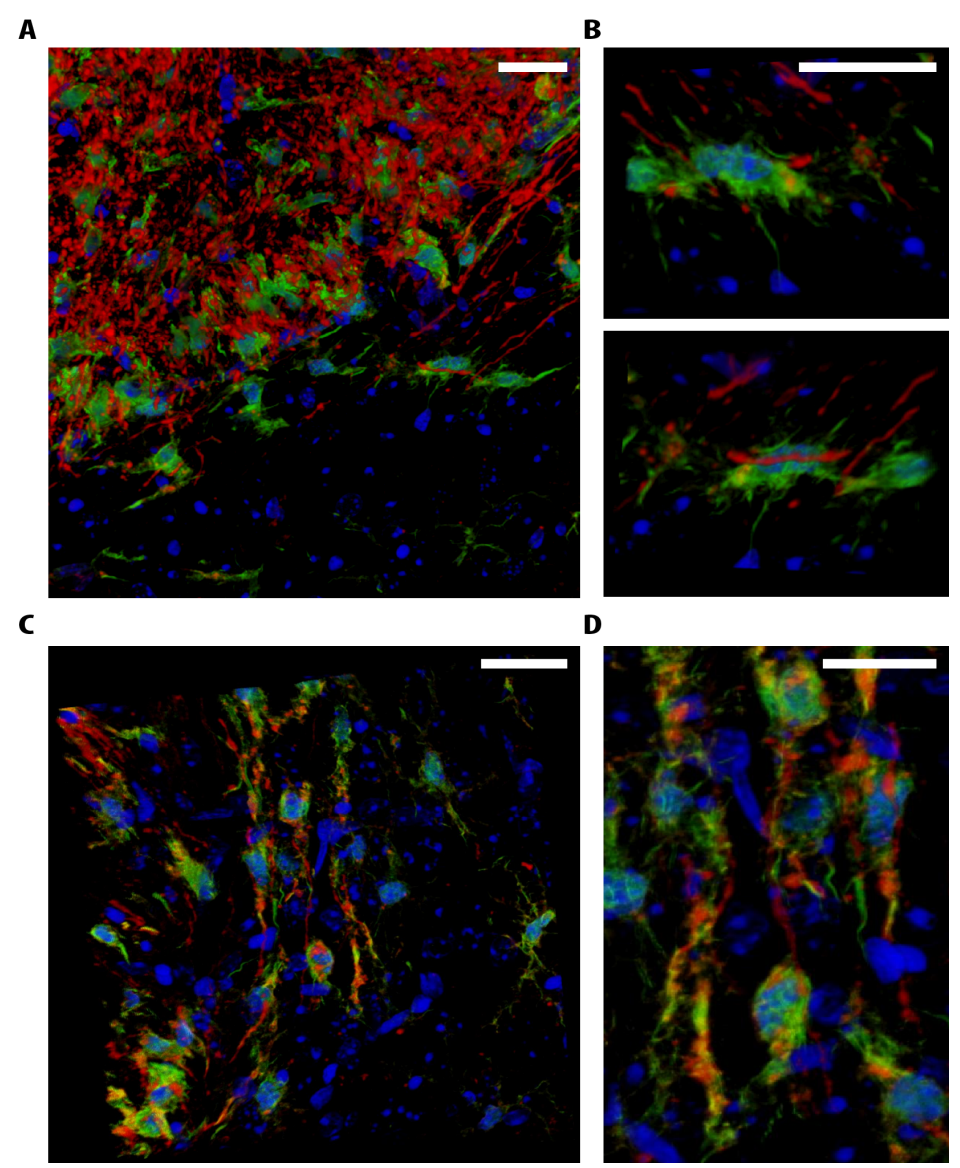

Figure 4.2: Microglia engulf QD9 positive material and are in association with QD9 positive fibers in the corpus callosum of cuprizone treated mice

Brain sections of cuprizone treated mice were stained for Iba1 (in green) and QD9 (in red). At 3 weeks of $0.2 \%$ cuprizone treatment, a high number of microglia was detected in the caudal corpus callosum (see A and C for over view). Magnification and 3D reconstruction revealed that the microglia engulf QD9 positive material (in B, same cell rotated in a $90^{\circ}$ angle around the vertical axis) and can be found in association with QD9 positive fibers (in D) (scale bar $25 \mu \mathrm{m}$ ). 


\subsubsection{Inner tongue as initiation site for myelin pathology}

In all demyelinating animal models assessed, the initial myelin pathology was detected in the adaxonal space with an enlarged inner tongue and its vesiculation. The adaxonal space is a relative cytoplasmic-rich compartment that is connected with the cell body via cytoplasmic channels and the paranodes (see Figure 4.3) [34] [368]. Metabolic disturbances caused by the long distance from the cell body and the high energy demand might render the inner tongue vulnerable [369].

As changes in the ion homeostasis leads to a decrease in the binding of MBP to the membrane, this cytoplasm-rich compartment is affected first. The decreased adhesion of the leaflets induces the vesiculation of the leaflets that is energetically more favorable. Once, the MBP network was assaulted, it seems to unzip the major dense line progressively. It was shown for the cuprizone model that even if the toxic diet was terminated after the first week the axons still demyelinated [260]. This finding suggests that once the myelin pathology is induced, it cannot be reverted. As cuprizone induces apoptosis of ODC, the myelin sheath can survive long before fragmentation, but it will eventually be degraded. In order to assess whether the myelin pathology is reversible, one would have to chose a treatment that does not kill the ODC. This question is of vital importance and should be assessed also in the context whether the restoration of the myelin sheath can be induced. 


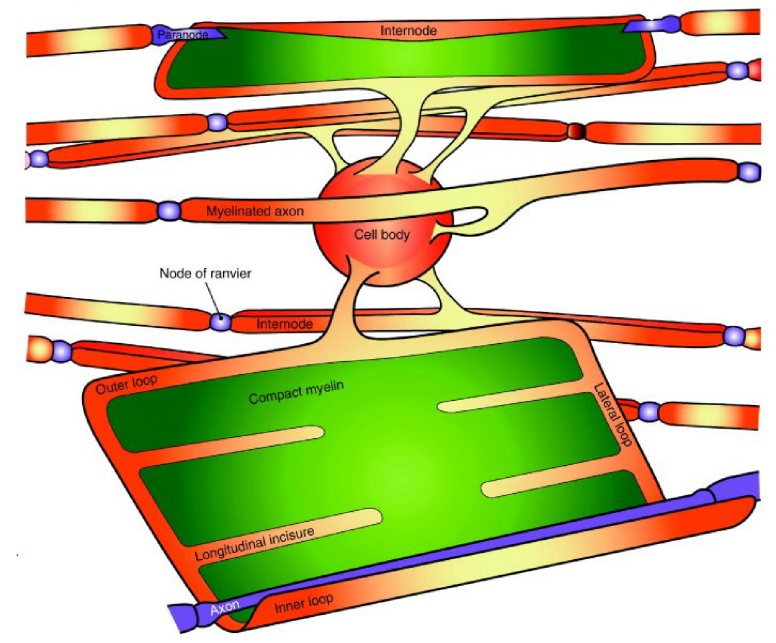

Figure 4.3: Cytoplasmic domains of mature oligodendrocytes Oligodendrocytes extend multiple processes that enwrap different axons and form a mature internode. The mature myelin sheath is depicted in its unwrapped state with the compact myelin areas marked in green. At the borders of the compact myelin, continuous cytoplasmic channels form the paranodal loops, the ad- and abaxonal space. Figure modified from [28].

\subsection{Causes of Myelin Degradation}

Many of the demyelinating animal models either cause the death of the ODC (e.g. cuprizone or virus infection) or directly attack the sheath as for anti-MOG or anti-Galc antibodies. One common mechanism in the early stages of the molecular disassembly of myelin seems to be an increase in intracellular calcium. Albeit, calcium is a very general second messenger that ODC's maintain at a low concentration [370]. Increases in calcium ions can be caused also by the binding of anti-myelin antibodies (such as antiGalc and anti-sulfatide [371] [146]) that leads to a sustained calcium influx and disruption of the MBP network in vitro. Furthermore, it is known that increased intracellular calcium leads to activation of calcium-dependent proteases, such as caspases and eventually, apoptosis.

Additionally, other stimuli such as ischemia cause an initial axonal injury and ultimately results in a non-synaptic release of glutamate in the extracellular space, where glutamate can activate AMPA/kainate receptors on ODC's. The activation of these non-NMDA ionotrophic receptors can directly damage the myelin sheath and injure the ODC. The calcium increase in the compact myelin in ischemia models can be blocked by AMPA receptor antagonist GYKI52466 and NBQX [372]. Furthermore, several 
groups showed that the systematic administration of NBQX during EAE has a beneficial effect on the clinical outcome. Antagonizing the AMPA/kainate receptor signaling resulted in an reduced myelin pathology and was further suggested to have a secondary protective effect on axons during ischemia [373].

Supportive data for the relevance of calcium in myelin pathology also comes from studies on the peripheral nerve and our own studies on acute brain slices, where the vesiculation of the myelin membrane was induced by calcium ionophore Ionomycin [360]. In both studies, the calcium chelator EGTA was shown to abolish myelin fragmentation ex vivo. Hence, calcium seems to play a key role in the initiation of the molecular disassembly of myelin. Conclusively, my data and the data from the literature highlight the detrimental effect of increased calcium influx in the ODC. Moreover, these results shed light on the need for pharmaceutical agents that block calcium influx specifically in ODC prior to demyelination.

\subsection{Downstream effects of calcium infl ux}

The increase of the intracellular calcium concentration in ODC's has various effects that promote the disassembly of proteins and lipids and finally, the fragmentation of compact myelin. Within this thesis, I aim to briefly highlight some of the many possible influences calcium influx can have on myelin disassembly.

Calcium influx can lead to activation of phospholipase $\mathrm{C}$ that mediates the hydrolysis of PIP2. This negatively charged lipid is an important binding partner of MBP and its degradation causes MBP to detach from the membrane [348]. This dissociation of MBP from the membrane renders the protein accessible to post-translation modifications and proteolytic degradation [131].

Post-translational modifications of MBP implicated in demyelinating diseases are mainly phosphorylation and citrullination. In MS, MBP exhibits a decreased phosphorylation that was associated with a loss of $\beta$-sheets and hence, a change in secondary structure [123] [119]. The detected increased citrullination of MBP in MS by calcium-sensitive peptidylarginine deiminase (PAD) [124] is thought to exhibit a decreased membrane binding and cause vesiculation of multilamellar vesicles as shown in vitro [374]. Furthermore, the decrease in positive charge of citrunillated MBP could be correlated with the presence of less compact myelin [375] and even with the severity of MS [131]. Citrunillated MBP was shown to form a shorter and more surfaceexposed amphipathic helix that leads to a faster digestion by proteases.

Calcium-dependent activation of neutral proteinases degrades preferentially MBP (as compared to PLP) [376]. Specifically, calpain was shown to medi- 
ate the proteolysis of MBP in a calcium dependent manner and was found to be increased in lesions of MS patients [377]. Additionally, acid proteinases such as cathepsin D were detected in MS tissue with increased activity [130] [376] and cleave especially citrullinated MBP within the amphipathic helices (reviewed in [131]). The proteolytic cleavage then exposes the immunodominant epitope that allows $\mathrm{T}$ cell recognition in the MHC class II context. Taken together, the calcium influx enables post-translational modifications of MBP that enhance its proteolytic digest and initiate the immune response.

The adhesion of proteins to the membrane is always determined by hydrophobic and electrostatic interactions that mainly depend on the lipid composition and its properties in terms of charge and fluidity. Changes of the lipid composition were reported for the EAE model and unraveled an increased lipid polyunsaturation, as well as an increased content of negatively charged PS, while SM, PC and sulfatide decrease. The EAE lipid composition allows an increased lipid phase fluidity and curvature favouring a decreased adhesion of the myelin leaflets and inducing vesiculation [378]. The lipid composition does not only affect the membrane itself, but also the binding of peripheral proteins. In myelin, proteins and lipids exert a mutual regulation in a cooperative way that perturbation of one affects the other [83], e.g. the adhesion of MBP is dependent on the surface charges of the lipid bilayer. Thus, the altered lipid composition in EAE initiates changes in biophysical properties and the myelin pathology. So far, it could not be elucidated, when the changes in lipid composition occur.

Calcium influx can also be caused by other cell types through detrimental soluble factors: Infiltrating macrophages were also shown to produce complement components and the membrane attack complex that can lead to the influx of various proteases and calcium. Neutral proteases and phosholipases released by the macrophages and potentiate myelin destruction [379]. Furthermore, the C9 component of the complement cascade was detected in EAE and MS tissue [380]. More surprisingly, terminal complement complexes were detected on the myelin sheath and ODC's prior to the onset of demyelination [381]. Thus, it would certainly be interesting to investigate what and when the formation of this membrane attack complex is triggered that has a detrimental effect on the myelin sheath. 


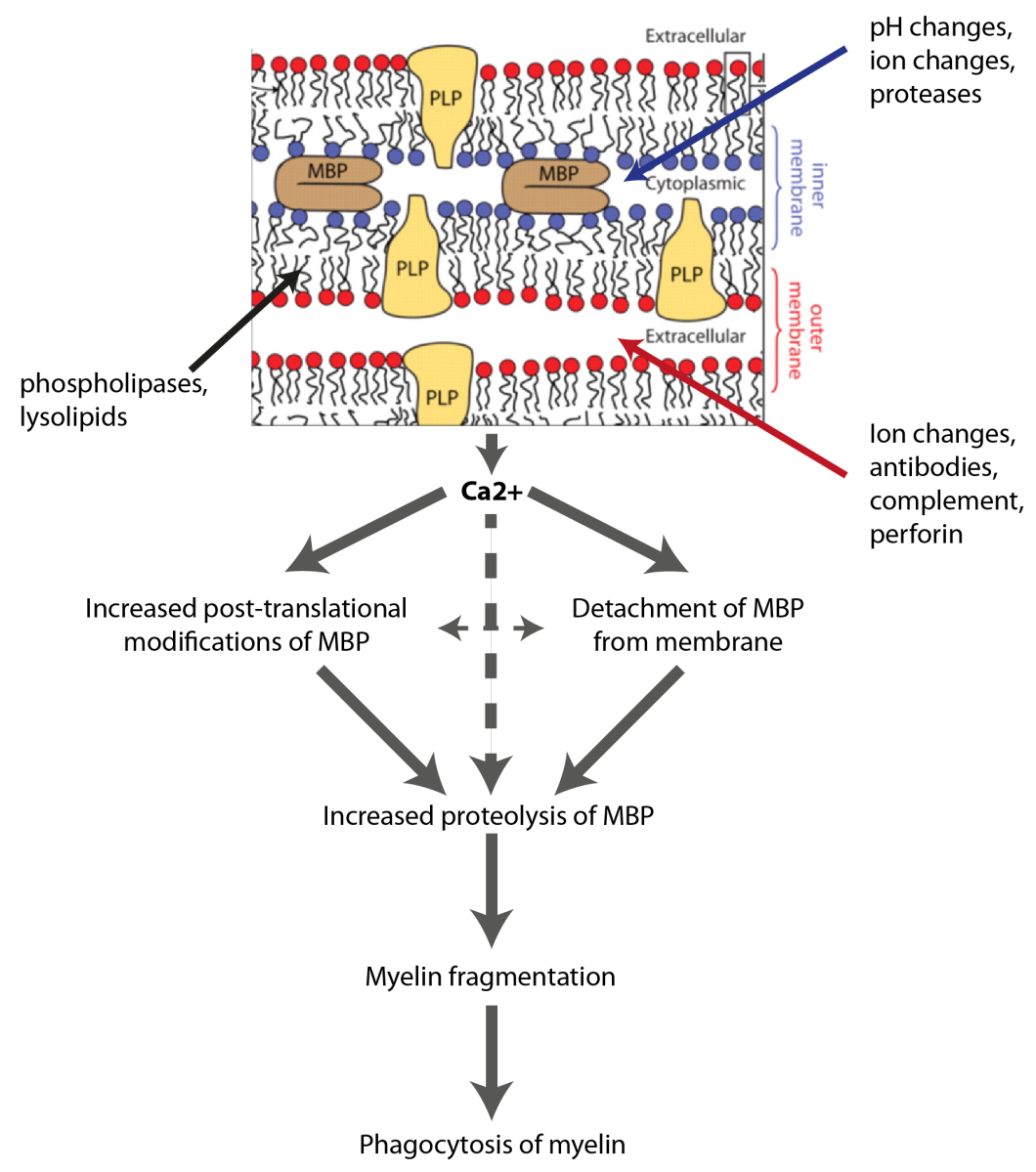

Figure 4.4: Molecular disassembly of myelin

Different possible causes of myelin degeneration are indicated with their target structure in the lipid bilayer (black arrow), in the outer membrane (red arrow) and in the inner membrane (blue arrow). One potential mechanism for the effect on MBP and on the whole myelin sheath is depicted below. Figure modified from [28].

\subsection{Reverse phase transition of MBP}

MBP is so far the only known protein necessary for compaction of the cytoplasmic leaflets in compact myelin [88]. Due to its basic charges, this naturally unstructured protein requires negatively charged lipids to bind the membrane. The interaction with the membrane allows MBP to acquire a secondary structure that enables it to self-interact and form a sieve-like network. Hence, it creates a network that restricts the entry for proteins with a big cytosolic domain [10]. This process was found to be mediated by hydrophobic interactions of phenylalanines within the amphipathic helices 
with the membrane. As a result, MBP is considered to undergo a phase transition from a dispersed state of single molecules to a cohesive network. Hence, MBP creates a liquid-liquid phase separation to a domain with distinct protein and lipid composition.

Phase transitions are frequently described for intrinsically unstructured proteins (IUP) with long, flexible connections between the binding motifs [343]. The modest affinity between low complexity sequences of these proteins often allow dynamic, reversible interactions [344] [345] [382]. The switch-like formation of these liquid compartments results in domains with a slowed diffusion rate and non-linear signaling pathways [343] [382] [383] [383] such as proteins of the nuclear pore complex[344] [345]. Strikingly, many IUP's were implicated in different neurodegenerative diseases, e.g. accumulation of tau leads to frontotemporal dementia and synuclein aggregates cause multiple system atrophy [384] [385] [386]. For some IUP's, such as RNA granule-like proteins, a reversible transformation from the soluble to a polymeric state was observed [382]. For other IUP's, mutations in proteins leads to the acceleration of the phenotype due to an irreversible transformation. For example, the mutation of the amyotrophic lateral sclerosis protein FUS leads to the formation of a irreversible hydrogel with impaired RNP granule function I could show that MBP exists in two states, namely the membrane-bound state of MBP and the dysfunctional single MBP molecules. In vitro studies inducing dysfunctional MBP in primary ODC's could show that changes in the membrane environment (e.g. depletion of specific type of lipids) can cause the detachment of MBP from the membrane. The disruption of the interaction with the membrane seems to also abolish the self-interaction of the MBP molecules. Furthermore, the dissociation from the membrane renders MBP dysfunctional as it cannot compact the membrane anymore allowing proteins with a bulky cytosol like CNP to enter the MBP positive areas.

In order to carry out its compaction function, MBP need to bind the membrane to be able to self-interact. I could show that the amphipathic helices in MBP are crucial for the molecular interactions with the membrane and formation of the MBP network. Interestingly, the QD9 antibody can detect dysfunctional MBP specifically binds an epitope adjacent to one of the amphipathic helices of MBP. This antibody was shown to specifically detect degenerated myelin in MS and Multiple System Atrophy patients [387] [347]. I could show with in vitro studies that treatments abolishing the membrane binding or function of MBP unmasked the QD9 epitope. Thus, the QD9 antibody can be used as a marker to distinguish the different MBP states depending on their membrane binding. Histological analysis of our cuprizone and focal NMO model revealed that this antibody epitope is unmasked already at very early time points prior to demyelination. Correlative studies could be applied that suggest a correlation of the QD9 staining with myelin pathology in EM. Taken together, the QD9 antibody can be used as a marker for dysfunctional MBP that lost its membrane binding and is 
implicated in early stages of myelin pathology.

Conditions that cause MBP to dissociate from the membrane (see above in section 3.4) seem to lead to a disassembly of the MBP network. The disruption of the major dense line seems to be the reason for the vesiculation of the myelin leaflets in early stages of myelin pathology. This finding is supported by the fact that these vesicles are devoid of MBP. Hence, the gradual loss of adhesion of MBP to the membrane leads to a destabilization of the myelin stacks and thus, initiates myelin vesiculation. Strikingly, the vesiculation of the innermost myelin lamellae manifest at the histological level in a loss of MAG staining in our studies, but also in lesions of NMO patients [294]. The trigger that causes MBP to lose its membrane adhesion seems to be mediated by calcium [348]. This second messenger signal triggers a whole range of effects that abolish the binding of MBP to the membrane and renders it dysfunctional before it is degraded. The disintegration of the MBP network into single molecules renders the myelin membrane stacks instable and hence, the lipid membranes are degraded.

In conclusion, the reverse phase transition of MBP from a cohesive network into single soluble protein molecules initiates the disassembly of myelin. Dysfunctional MBP molecules and the early pathological states of myelin can be marked with the QD9 antibody.

Taken together, this thesis sheds light on the molecular mechanisms of myelin disassembly and showed that the loss of function of MBP is the key event for initiation of myelin pathology. 


\section{Chapter 5}

\section{Summary}

"The beginning of knowledge is the discovery of something we do not understand."

- Frank Herbert

Myelin is the most frequent target of autoimmune diseases of the central nervous system. The scope of this thesis was to investigate the breakdown of myelin in demyelinating diseases and elucidate the underlying mechanism of myelin pathology (see Figure 5.1).

With the help of several models of demyelination, such as the toxin-mediated cuprizone and lysolecithin models, as well as antibody-mediated models employing EAE or focal lesions with anti-AQP4 or anti-MOG antibodies, I could identify a common sequence of myelin fragmentation: Initially, the innermost myelin lamellae become vesiculated before the pathology progressively encompasses the entire myelin sheath. The vesicules were detected preferentially in the adaxonal space and are derived from compact myelin as they contain PLP, but are devoid of MBP. The axonal damage became evident at the ultrastructural level only after severe vesiculation of myelin. Additionally to the common patterns of myelin breakdown, I could also detect myelin bulb formation, as well as split and vesiculated myelin formation in direct immune-mediated myelin damage. In conclusion, the vesiculation of the inner myelin layers seems to be a common mechanism for myelin breakdown in demyelinating diseases.

On an immunohistochemical level, the vesiculation of the inner myelin layers leads to a loss of the adaxonal protein MAG and only upon progressive myelin fragmentation, a loss of MBP could be detected. Furthermore, the loss of MAG in human NMO lesions was associated with a high number of infiltrating inflammatory cells. In contrast to that, when MBP was devoid 
from late stage lesions, a decreased number of immune cells could be detected. Hence, staining for MBP, MAG and inflammatory cells allows for the staging of the demyelinating lesions also in human samples.

In order to elucidate the underlying mechanism of the myelin breakdown, I focused on MBP as the essential protein for myelin compaction. MBP molecules can undergo a phase transition from a single, soluble state to a cohesive network that is able to compact the lipid membranes. This phase transition can be reverted to yield dysfunctional MBP molecules in vitro by changes in the lipid composition (mediated by Fumonisine B1 or methyl$\beta$-cyclodextrin), as well as clustering of lipids via anti-lipid antibodies. I could show that these treatments lead to the loss of the membrane binding of MBP as indicative by the unmasking of PIP2 that is strongly associated with MBP in normal conditions. Furthermore, the resulting dysfunctional $\mathrm{MBP}$ is not able to compact the lipid membranes any longer as shown by the intermixing of MBP and CNP that are normally clearly separated. Additionally, this result was supported by a FRET assay indicating the loss of self-interactions between MBP molecules. Hence, changes in the lipid composition induce the reverse phase transition and thus, a loss of function of MBP.

To assess if MBP loses its adhesive and self-interacting function in early stages of demyelinating animal models, I employed the QD9 antibody that specifically detects the pool of dysfunctional MBP molecules. Myelin fragmentation in different antibody- or toxin-mediated models could be correlated with an increase in QD9 staining. Hence, suggesting that a phase transition of MBP from a cohesive network to a non-adhesive, dispersed state is the key event to initiate myelin fragmentation. Additionally, the QD9 antibody could be shown to stain border zones of chronic white matter lesions with ongoing inflammation as indicated by CD68-positive cells in Multiple Sclerosis patients.

Moreover, I could show that this reverse phase transition and the resulting myelin vesiculation can be triggered by calcium influx. When the increase of intracellular calcium is abolished, the induction of dysfunction MBP molecules is prevented. So far, our studies suggest calcium as a trigger for demyelination, but further studies might discover new molecules involved in the mechanism, which, if inhibited, will slow down or block the loss of myelin.

This thesis sheds light on the underlying mechanism involving the aberrant phase transition of MBP that triggers myelin fragmentation in early stages of demyelinating diseases. 
Normal state
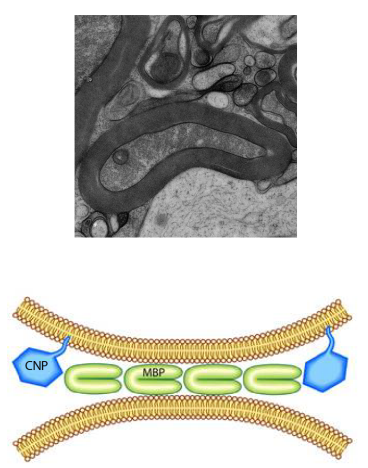

Cohesive MBP network

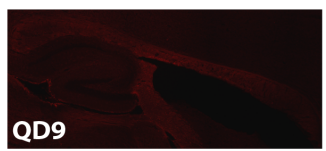

Pathological state
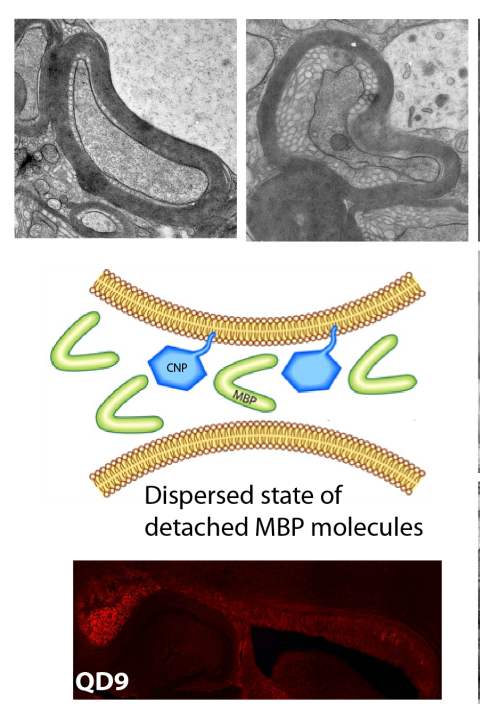

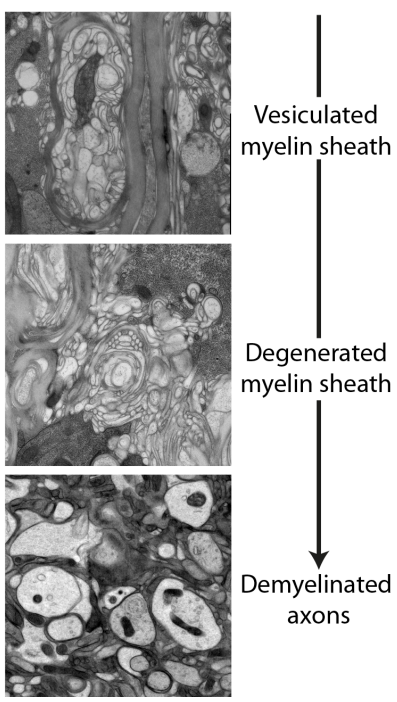

Figure 5.1: Correlation of myelin ultrastructure with QD9 staining revealing different structural states of MBP under normal and pathological conditions

In the left panel, the myelin ultrastructure with its alternating electron dense and light lines is depicted as it occurs under normal conditions, where MBP molecules assemble into a cohesive network. Due to masking of the QD9 epitope in this membrane bound state of MBP, hardly any QD9 staining is obtained. Under pathological conditions as depicted in the right panel, the inner tongue becomes vesiculated progressively (see pictures from left to right) before the membrane stacks degenerated and the axons becomes demyelinated. During this time course, MBP detaches from the membrane and is rendered soluble. Therefore, the QD9 epitope becomes unmasked and its staining efficiency increases intensively. 

Appendix 


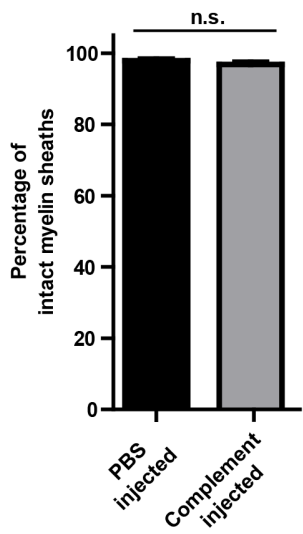

Figure 5.2: Acute axonal damage in focal NMO

Lewis rats were injected in the corpus callosum with complement alone or PBS. Myelin fragmentation was quantified and did not significantly differ between the two groups. Bars show mean with SEM $\left(\mathrm{n}=3,{ }^{* *}=\mathrm{P}<0.01\right.$, two-tailed t-test $)$.

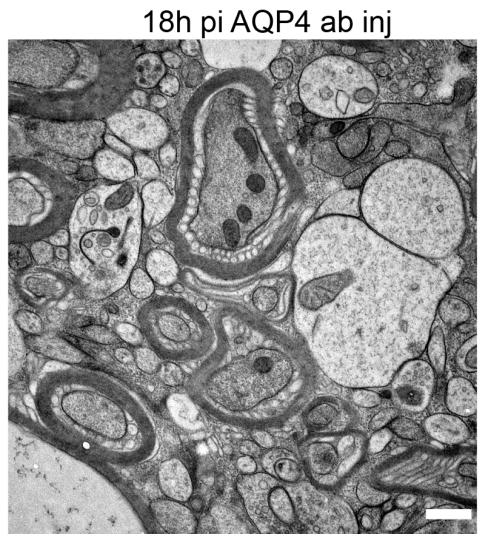

5d pi AQP4 ab inj

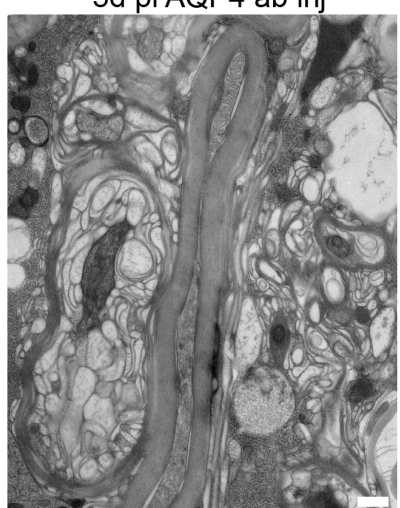

b

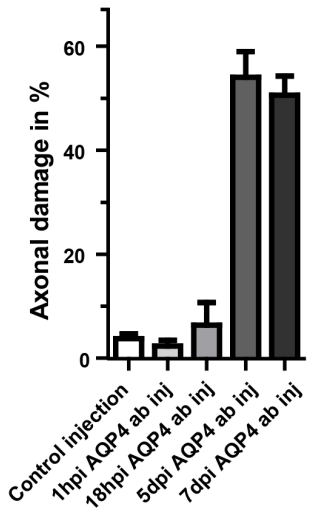

Figure 5.3: Acute axonal damage in focal NMO

Lewis rats were injected in the corpus callosum with AQP4 antibody and complement or complement alone (scale bar $500 \mathrm{~nm}$ ). Representative electron micrographs of focal NMO lesions at $18 \mathrm{~h}$ and $5 \mathrm{~d}$ p.i. are shown in A. The axonal damage was quantified based on the dark contrast and the loss of cytoskeletal proteins, as well as axonal swellings. This quantification is depicted in B for the whole time course investigated. Bars show mean with SEM $\left(\mathrm{n}=3,{ }^{* *}=\mathrm{P}<0.01\right.$, two-tailed t-test $)$. 


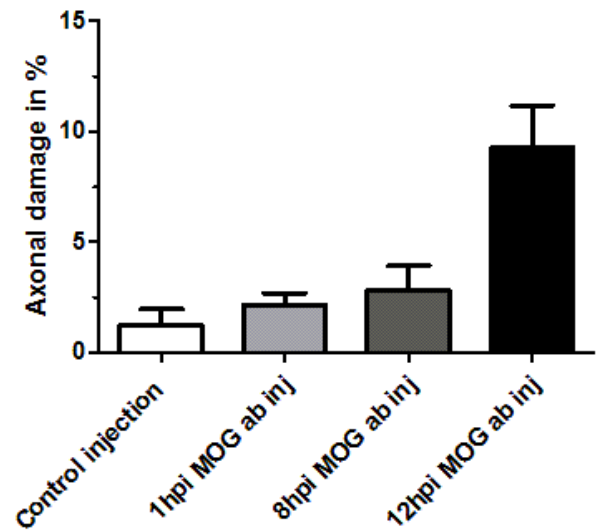

Figure 5.4: Acute axonal damage in MOG-antibody induced lesions

Lewis rats were injected in the corpus callosum with MOG antibody and complement or PBS (scale bar $500 \mathrm{~nm}$ ). The axonal damage was quantified based on the dark contrast and the loss of cytoskeletal proteins, as well as axonal swellings. Bars show mean with $\operatorname{SEM}(\mathrm{n}=3, \mathrm{n} . \mathrm{s} .=$ not significant, two-tailed t-test).

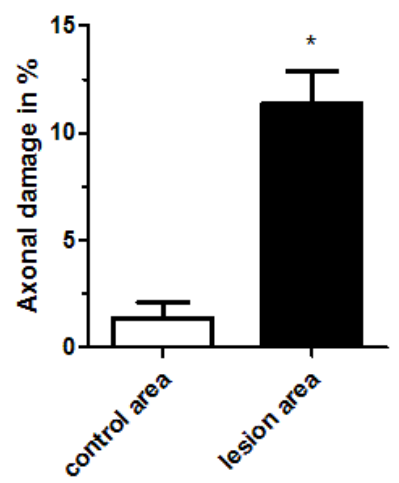

Figure 5.5: Acute axonal damage in EAE lesions

Axonal damage at the borders of demyelinating EAE lesions was quantified. Bars show mean with SEM $(\mathrm{n}=3, *=\mathrm{P}<0.1$, two-tailed t-test $)$. 
Table 5.1: Generated plasmids

\begin{tabular}{|c|c|c|c|c|c|}
\hline Plasmid name & Insert details & Size & Resistance & Sequencing primers & Backbone \\
\hline MBP $14 \mathrm{kD}$ & $\begin{array}{lr}\text { MOG Signal } \\
\text { peptide, myc } \\
\text { tag, MBP } 14 \\
\text { kDa WT }\end{array}$ & $550 \mathrm{bp}$ & ampicillin & $620 / 621$ & pcDNA \\
\hline $\begin{array}{l}\mathrm{MBP} 14 \mathrm{kD} \mathrm{F} \\
\rightarrow \mathrm{S}\end{array}$ & $\begin{array}{l}\text { MOG Signal } \\
\text { peptide, myc } \\
\text { tag, MBP } 14 \\
\mathrm{kDa} \mathrm{F} \rightarrow \mathrm{S}\end{array}$ & $550 \mathrm{bp}$ & ampicillin & $620 / 621$ & pcDNA \\
\hline $\begin{array}{l}\text { MBP } 14 \mathrm{kD} \mathrm{F} \\
1,6 \rightarrow \mathrm{S}\end{array}$ & $\begin{array}{l}\text { MOG Signal } \\
\text { peptide, myc } \\
\text { tag, MBP } 14 \\
\text { kDa F } 1,6 \rightarrow \mathrm{S}\end{array}$ & $550 \mathrm{bp}$ & ampicillin & $620 / 621$ & pcDNA \\
\hline $\begin{array}{l}\text { MBP } 14 \mathrm{kD} \mathrm{F} \\
2,3 \rightarrow \mathrm{S}\end{array}$ & $\begin{array}{l}\text { MOG Signal } \\
\text { peptide, myc } \\
\text { tag, MBP } 14 \\
\text { kDa F } 2,3 \rightarrow \mathrm{S}\end{array}$ & $550 \mathrm{bp}$ & ampicillin & $620 / 621$ & pcDNA \\
\hline $\begin{array}{l}\mathrm{MBP} 14 \mathrm{kD} \mathrm{F} \\
4,5 \rightarrow \mathrm{S}\end{array}$ & $\begin{array}{l}\text { MOG Signal } \\
\text { peptide, myc } \\
\text { tag, MBP } 14 \\
\text { kDa F } 4,5 \rightarrow \mathrm{S}\end{array}$ & $550 \mathrm{bp}$ & ampicillin & $620 / 621$ & pcDNA \\
\hline $\begin{array}{l}\text { MBP } 14 \mathrm{kD} \mathrm{F} \\
2,3,4,5 \rightarrow \mathrm{S}\end{array}$ & 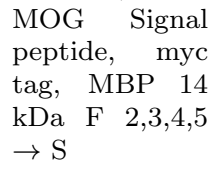 & $550 \mathrm{bp}$ & ampicillin & $620 / 621$ & pcDNA \\
\hline $\begin{array}{l}\text { MBP 14 } 14 \text { kD } \\
\mathrm{F} 2,3 \rightarrow \mathrm{S} \\
(\mathrm{pSF} 2279)\end{array}$ & $\begin{array}{l}\text { MBP } 14 \mathrm{kDaF} \\
2,3 \rightarrow \mathrm{S} \text { with } \\
\mathrm{N}-\text { terminal } \\
\text { His-tag and } \\
\text { C-terminal } \\
\text { Cysteine for } \\
\text { protein purific- } \\
\text { ation }\end{array}$ & $500 \mathrm{bp}$ & kanamycin & 492 & pQE \\
\hline $\begin{array}{l}\text { MBP } 14 \quad \mathrm{kD} \\
\mathrm{F} \quad 4,5 \rightarrow \mathrm{S} \\
(\mathrm{pSF} 2284)\end{array}$ & $\begin{array}{l}\mathrm{MBP} 14 \mathrm{kDa} F \\
4,5 \rightarrow \mathrm{S} \text { with } \\
\mathrm{N}-\text { terminal } \\
\text { His-tag and } \\
\text { C-terminal } \\
\text { Cysteine for } \\
\text { protein purific- } \\
\text { ation }\end{array}$ & $500 \mathrm{bp}$ & kanamycin & 492 & pQE \\
\hline $\begin{array}{l}\text { MBP 14 kD } \\
\text { F 2,3,4,5 } \rightarrow \mathrm{S} \\
(\mathrm{pSF} 2300)\end{array}$ & $\begin{array}{l}\text { MBP } 14 \text { kDa } \\
\mathrm{F} \quad 2,3,4,5 \\
\rightarrow \quad \mathrm{S} \text { with } \\
\text { N-terminal } \\
\text { His-tag and } \\
\text { C-terminal } \\
\text { Cysteine for } \\
\text { protein purific- } \\
\text { ation }\end{array}$ & $500 \mathrm{bp}$ & kanamycin & 492 & pQE \\
\hline
\end{tabular}




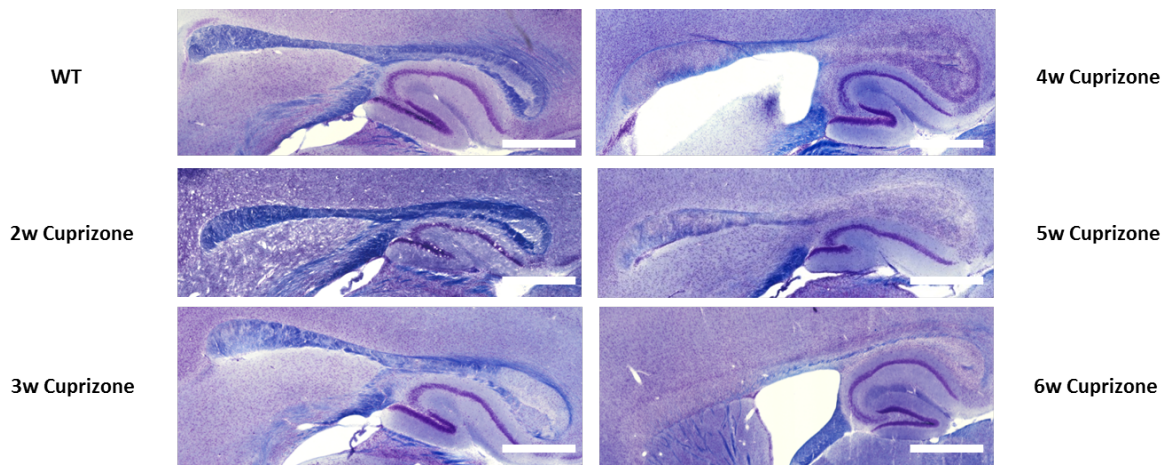

Figure 5.6: Luxol Fast blue staining of cuprizone time course

C57B6 mice were treated with $0.2 \%$ of cuprizone and each week for 6 weeks, one set of mice was processed for immunohistochemistry and subsequently stained with Luxol Fast blue (LFB) and cresyl violet. Loss of LFB stain can already be detected after 3 weeks of cuprizone in the caudal corpus callosum. After further exposure (4 to 6 weeks cuprizone), the whole corpus callosum lost the LFB stain that is indicative for demyelination in these areas (scale bar $1 \mathrm{~mm}$ ). 
A

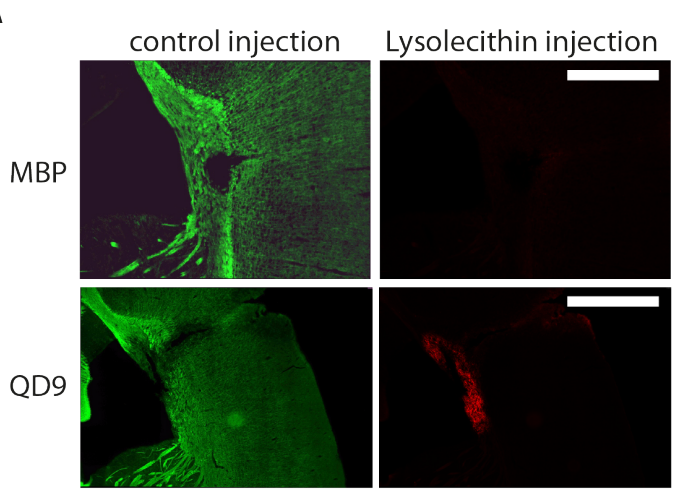

B

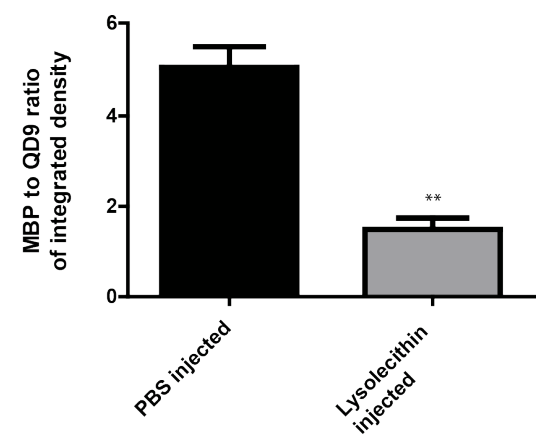

C

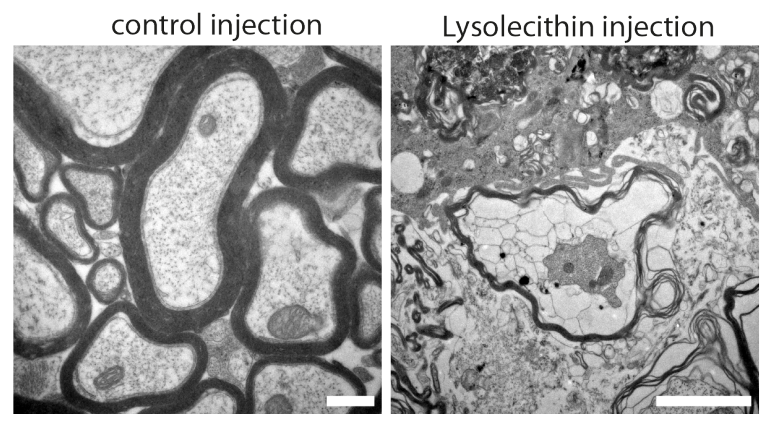

Figure 5.7: Myelin pathology in Lysolecithin injections

C57B6 mice were stereotactically injected in the CC with $0.1 \%$ of lysolecithin and the lesion site was assessed after $7 \mathrm{dpi}$. The brain lesions were stained with MBP (in green) and QD9 (in red) as shown in A (scale bar $1 \mathrm{~mm}$ ). The MBP to QD9 ratio was quantified in B. Bars show mean with SEM $\left(\mathrm{n}=3,{ }^{* *}=\mathrm{P}<0.01\right.$, two-tailed t-test). Representative images of electron micrographs of the control and lysolecithin lesions are depicted in $\mathrm{C}$ (scale bar $500 \mathrm{~nm}$ ). 
a

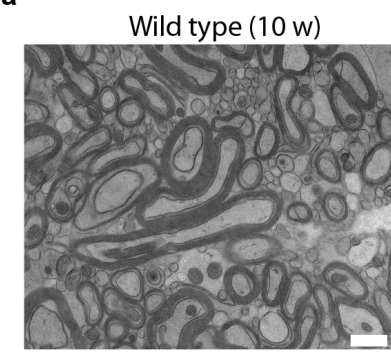

b

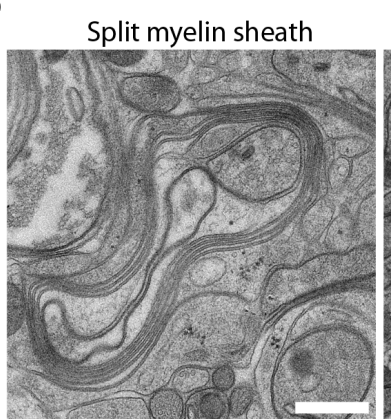

Shiverer $(10 \mathrm{w})$

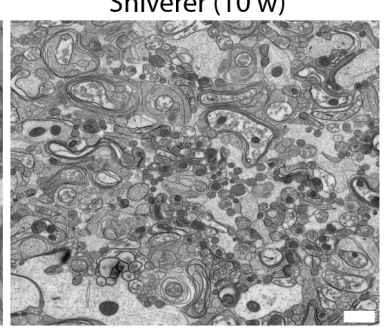

vesiculated myelin sheath

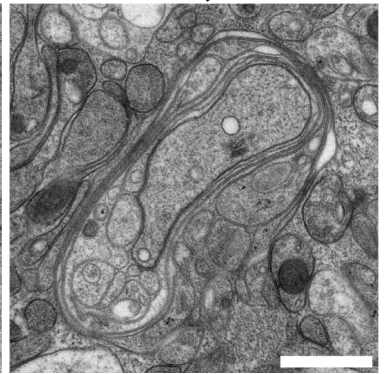

C

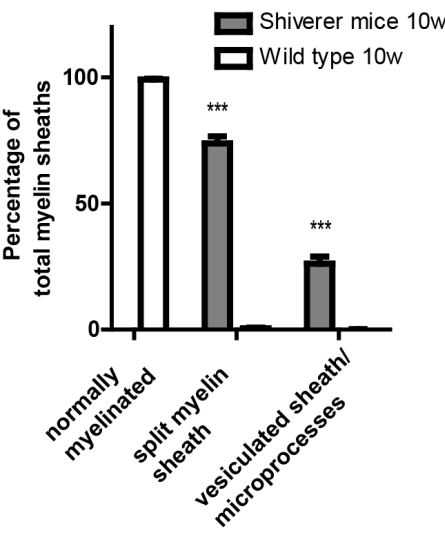

Figure 5.8: Fragmentation of myelin in shiverer mice

Representative electron micrographs of shiverer mice and age-matched wild-type mice are depicted in $\mathrm{A}$ and $\mathrm{B}$ respectively with a magnification of the myelin pathology detected in the panel below (scale bar $500 \mathrm{~nm}$ ). The split or vesiculated myelin sheaths (including microprocesses) were quantified as percentage of total myelin sheaths in B. Bars show mean with SEM $(\mathrm{n}=3, * * *=\mathrm{P}<0.001$, two-way ANOVA). 



\section{Bibliography}

[1] N. J. Allen and B. A. Barres. Neuroscience: Glia - more than just brain glue. Nature, 457(7230):675-7, feb 2009.

[2] T. Kandel, E.R.; Schwartz, J.H.; Jessel. Principle of Neural Sciences. McGraw-Hill, 2000.

[3] O. Jahn, S. Tenzer, and H. B. Werner. Myelin proteomics: molecular anatomy of an insulating sheath. Mol. Neurobiol., 40(1):55-72, aug 2009.

[4] M. B. BUNGE, R. P. BUNGE, and G. D. PAPPAS. Electron microscopic demonstration of connections between glia and myelin sheaths in the developing mammalian central nervous system. J. Cell Biol., 12:448-53, feb 1962.

[5] K.-A. Nave. Myelination and the trophic support of long axons. Nat. Rev. Neurosci., 11(4):275-83, apr 2010.

[6] D. K. Hartline and D. R. Colman. Rapid conduction and the evolution of giant axons and myelinated fibers. Curr. Biol., 17(1):R29-35, jan 2007.

[7] R. Reynolds and G. P. Wilkin. Development of macroglial cells in rat cerebellum. II. An in situ immunohistochemical study of oligodendroglial lineage from precursor to mature myelinating cell. Development, 102(2):409-25, feb 1988.

[8] A. Compston, J. Zajicek, J. Sussman, A. Webb, G. Hall, D. Muir, C. Shaw, A. Wood, and N. Scolding. Glial lineages and myelination in the central nervous system. $J$. Anat., 190 ( Pt 2:161-200, feb 1997.

[9] J. M. Levine, R. Reynolds, and J. W. Fawcett. The oligodendrocyte precursor cell in health and disease. Trends Neurosci., 24(1):39-47, jan 2001.

[10] S. Aggarwal, N. Snaidero, G. Pähler, S. Frey, P. Sánchez, M. Zweckstetter, A. Janshoff, A. Schneider, M. T. Weil, I. A. T. Schaap, D. Görlich, and M. Simons. Myelin Membrane Assembly Is Driven by a Phase Transition of Myelin Basic Proteins Into a Cohesive Protein Meshwork. PLoS Biol., 11(6), 2013.

[11] S. C. Zhang. Defining glial cells during CNS development. Nat. Rev. Neurosci., 2(11):840-3, nov 2001.

[12] K. M. Young, K. Psachoulia, R. B. Tripathi, S.-J. Dunn, L. Cossell, D. Attwell, K. Tohyama, and W. D. Richardson. Oligodendrocyte dynamics in the healthy adult CNS: evidence for myelin remodeling. Neuron, 77(5):873-85, mar 2013.

[13] S. H. Kang, M. Fukaya, J. K. Yang, J. D. Rothstein, and D. E. Bergles. NG2+ CNS glial progenitors remain committed to the oligodendrocyte lineage in postnatal life and following neurodegeneration. Neuron, 68(4):668-81, nov 2010.

[14] N. Baumann and D. Pham-Dinh. Biology of oligodendrocyte and myelin in the mammalian central nervous system. Physiol. Rev., 81(2):871-927, apr 2001. 
[15] R. D. Fields. White matter in learning, cognition and psychiatric disorders. Trends Neurosci., 31(7):361-70, jul 2008.

[16] M. A. Matthews and D. Duncan. A quantitative study of morphological changes accompanying the initiation and progress of myelin production in the dorsal funiculus of the rat spinal cord. J. Comp. Neurol., 142(1):1-22, may 1971.

[17] R. G. Almeida, T. Czopka, C. Ffrench-Constant, and D. A. Lyons. Individual axons regulate the myelinating potential of single oligodendrocytes in vivo. Development, 138(20):4443-4450, aug 2011.

[18] S. Remahl and C. Hildebrand. Changing relation between onset of myelination and axon diameter range in developing feline white matter. J. Neurol. Sci., 54(1):33-45, apr 1982 .

[19] S. G. Waxman and M. V. Bennett. Relative conduction velocities of small myelinated and non-myelinated fibres in the central nervous system. Nat. New Biol., 238(85):217-9, aug 1972.

[20] M. Siegel, G.j.; Agranoff, B.W.; Albers, R.W.; Fisher, S.K.; Uhler. Basic Neurochemistry. Lippincott Williams and Wilkins, 6th edition, 1998.

[21] W. A. H. RUSHTON. A theory of the effects of fibre size in medullated nerve. J. Physiol., 115(1):101-22, sep 1951.

[22] T. Chomiak and B. Hu. What is the optimal value of the g-ratio for myelinated fibers in the rat CNS? A theoretical approach. PLoS One, 4(11):e7754, jan 2009.

[23] S. G. Waxman. Determinants of conduction velocity in myelinated nerve fibers. Muscle Nerve, 3(2):141-50, jan 1980.

[24] J. A. Murray and W. F. Blakemore. The relationship between internodal length and fibre diameter in the spinal cord of the cat. J. Neurol. Sci., 45(1):29-41, feb 1980.

[25] C. Hildebrand, S. Remahl, H. Persson, and C. Bjartmar. Myelinated nerve fibres in the CNS. Prog. Neurobiol., 40(3):319-384, mar 1993.

[26] S. Pfeiffer, A. Warrington, and R. Bansal. The oligodendrocyte and its many cellular processes. Trends Cell Biol., 3(6):191-197, jun 1993.

[27] B. H. Toyama, J. N. Savas, S. K. Park, M. S. Harris, N. T. Ingolia, J. R. Yates, and M. W. Hetzer. Identification of long-lived proteins reveals exceptional stability of essential cellular structures. Cell, 154(5):971-82, aug 2013.

[28] S. Aggarwal, L. Yurlova, N. Snaidero, C. Reetz, S. Frey, J. Zimmermann, G. Pähler, A. Janshoff, J. Friedrichs, D. J. Müller, C. Goebel, and M. Simons. A size barrier limits protein diffusion at the cell surface to generate lipid-rich myelin-membrane sheets. Dev. Cell, 21(3):445-56, sep 2011.

[29] V. Bennett and S. Lambert. Physiological roles of axonal ankyrins in survival of premyelinated axons and localization of voltage-gated sodium channels. J. Neurocytol., 28(4-5):303-18, jan.

[30] S. Tait, F. Gunn-Moore, J. M. Collinson, J. Huang, C. Lubetzki, L. Pedraza, D. L. Sherman, D. R. Colman, and P. J. Brophy. An oligodendrocyte cell adhesion molecule at the site of assembly of the paranodal axo-glial junction. J. Cell Biol., 150(3):657-66, aug 2000.

[31] A. M. Pillai, C. Thaxton, A. L. Pribisko, J.-G. Cheng, J. L. Dupree, and M. A. Bhat. Spatiotemporal ablation of myelinating glia-specific neurofascin (Nfasc NF155) in mice reveals gradual loss of paranodal axoglial junctions and concomitant disorganization of axonal domains. J. Neurosci. Res., 87(8):1773-93, jun 2009.

[32] Y. Takai, K. Irie, K. Shimizu, T. Sakisaka, and W. Ikeda. Nectins and nectinlike molecules: roles in cell adhesion, migration, and polarization. Cancer Sci., 94(8):655-67, aug 2003. 
[33] S. Poliak and E. Peles. The local differentiation of myelinated axons at nodes of Ranvier. Nat. Rev. Neurosci., 4(12):968-980, dec 2003.

[34] N. Snaidero and M. Simons. Myelination at a glance. J. Cell Sci., 127(Pt 14):29993004, jul 2014.

[35] R. H. Quarles. Myelin-associated glycoprotein (MAG): past, present and beyond. J. Neurochem., 100(6):1431-48, mar 2007.

[36] C. Brunner, H. Lassmann, T. V. Waehneldt, J. M. Matthieu, and C. Linington. Differential ultrastructural localization of myelin basic protein, myelin/oligodendroglial glycoprotein, and 2',3'-cyclic nucleotide 3'-phosphodiesterase in the CNS of adult rats. J. Neurochem., 52(1):296-304, jan 1989.

[37] U. Fünfschilling, L. M. Supplie, D. Mahad, S. Boretius, A. S. Saab, J. Edgar, B. G. Brinkmann, C. M. Kassmann, I. D. Tzvetanova, W. Möbius, F. Diaz, D. Meijer, U. Suter, B. Hamprecht, M. W. Sereda, C. T. Moraes, J. Frahm, S. Goebbels, and K.-A. Nave. Glycolytic oligodendrocytes maintain myelin and long-term axonal integrity. Nature, 485(7399):517-21, may 2012.

[38] D.-H. Lee, E. Geyer, A.-C. Flach, K. Jung, R. Gold, A. Flügel, R. A. Linker, and F. Lühder. Central nervous system rather than immune cell-derived BDNF mediates axonal protective effects early in autoimmune demyelination. Acta Neuropathol., 123(2):247-58, feb 2012.

[39] K.-A. Nave and B. D. Trapp. Axon-Glial Signaling and the Glial Support of Axon Function. Annu. Rev. Neurosci., 31(1):535-561, jul 2008.

[40] I. Griffiths, M. Klugmann, T. Anderson, D. Yool, C. Thomson, M. H. Schwab, A. Schneider, F. Zimmermann, M. McCulloch, N. Nadon, and K. A. Nave. Axonal swellings and degeneration in mice lacking the major proteolipid of myelin. Science, 280(5369):1610-3, jun 1998.

[41] X. Yin, T. O. Crawford, J. W. Griffin, P. h. Tu, V. M. Lee, C. Li, J. Roder, and B. D. Trapp. Myelin-associated glycoprotein is a myelin signal that modulates the caliber of myelinated axons. J. Neurosci., 18(6):1953-62, mar 1998.

[42] C. Lappe-Siefke, S. Goebbels, M. Gravel, E. Nicksch, J. Lee, P. E. Braun, I. R. Griffiths, and K.-A. Nave. Disruption of Cnp1 uncouples oligodendroglial functions in axonal support and myelination. Nat. Genet., 33(3):366-74, mar 2003.

[43] T. Norton and L. A. Autilio. The chemical composition of bovine CNS myelin. Ann. N. Y. Acad. Sci., 122:77-85, mar 1965.

[44] W. T. Norton and S. E. Poduslo. Myelination in rat brain: Method of myelin isolation. J. Neurochem., 21(4):749-757, oct 1973.

[45] J. S. O'Brien and E. L. Sampson. Myelin Membrane: a molecular abnormality. Science, 150(3703):1613-4, dec 1965.

[46] M. Bohdanowicz and S. Grinstein. Role of phospholipids in endocytosis, phagocytosis, and macropinocytosis. Physiol. Rev., 93(1):69-106, jan 2013.

[47] M. O. Li, M. R. Sarkisian, W. Z. Mehal, P. Rakic, and R. A. Flavell. Phosphatidylserine receptor is required for clearance of apoptotic cells. Science, 302(5650):1560-3, nov 2003.

[48] J. F. Hancock, H. Paterson, and C. J. Marshall. A polybasic domain or palmitoylation is required in addition to the CAAX motif to localize p21ras to the plasma membrane. Cell, 63(1):133-9, oct 1990.

[49] A. A. Musse, E. Polverini, R. Raijmakers, and G. Harauz. Kinetics of human peptidylarginine deiminase 2 (hPAD2)-reduction of $\mathrm{Ca} 2+$ dependence by phospholipids and assessment of proposed inhibition by paclitaxel side chains. Biochem. Cell Biol., 86(5):437-47, oct 2008. 
[50] A. A. Farooqui, L. A. Horrocks, and T. Farooqui. Glycerophospholipids in brain: their metabolism, incorporation into membranes, functions, and involvement in neurological disorders. Chem. Phys. Lipids, 106(1):1-29, jun 2000.

[51] N. Nagan and R. A. Zoeller. Plasmalogens: biosynthesis and functions. Prog. Lipid Res., 40(3):199-229, may 2001.

[52] A. Teigler, D. Komljenovic, A. Draguhn, K. Gorgas, and W. W. Just. Defects in myelination, paranode organization and Purkinje cell innervation in the ether lipid-deficient mouse cerebellum. Hum. Mol. Genet., 18(11):1897-908, jun 2009.

[53] S. McLaughlin, J. Wang, A. Gambhir, and D. Murray. PIP(2) and proteins: interactions, organization, and information flow. Annu. Rev. Biophys. Biomol. Struct., 31:151-75, jan 2002.

[54] J. M. Haugh, F. Codazzi, M. Teruel, and T. Meyer. Spatial sensing in fibroblasts mediated by 3' phosphoinositides. J. Cell Biol., 151(6):1269-80, dec 2000.

[55] A. Simonsen, A. E. Wurmser, S. D. Emr, and H. Stenmark. The role of phosphoinositides in membrane transport. Curr. Opin. Cell Biol., 13(4):485-92, aug 2001.

[56] M. P. Czech. PIP2 and PIP3: complex roles at the cell surface. Cell, 100(6):603-6, mar 2000.

[57] A. S. Sechi and J. Wehland. The actin cytoskeleton and plasma membrane connection: PtdIns $(4,5) \mathrm{P}(2)$ influences cytoskeletal protein activity at the plasma membrane. J. Cell Sci., 113 Pt 21:3685-95, nov 2000.

[58] A. I. Flores, S. P. Narayanan, E. N. Morse, H. E. Shick, X. Yin, G. Kidd, R. L. Avila, D. A. Kirschner, and W. B. Macklin. Constitutively active Akt induces enhanced myelination in the CNS. J. Neurosci., 28(28):7174-83, jul 2008.

[59] S. Degroote, J. Wolthoorn, and G. van Meer. The cell biology of glycosphingolipids. Semin. Cell Dev. Biol., 15(4):375-87, aug 2004.

[60] R. G. Farrer and R. H. Quarles. GT3 and its O-acetylated derivative are the principal A2B5-reactive gangliosides in cultured O2A lineage cells and are down-regulated along with O-acetyl GD3 during differentiation to oligodendrocytes. J. Neurosci. Res., 57(3):371-80, aug 1999.

[61] S. Imgrund, D. Hartmann, H. Farwanah, M. Eckhardt, R. Sandhoff, J. Degen, V. Gieselmann, K. Sandhoff, and K. Willecke. Adult ceramide synthase 2 (CERS2)deficient mice exhibit myelin sheath defects, cerebellar degeneration, and hepatocarcinomas. J. Biol. Chem., 284(48):33549-60, nov 2009.

[62] O. Ben-David, Y. Pewzner-Jung, O. Brenner, E. L. Laviad, A. Kogot-Levin, I. Weissberg, I. E. Biton, R. Pienik, E. Wang, S. Kelly, J. Alroy, A. Raas-Rothschild, A. Friedman, B. Brügger, A. H. Merrill, and A. H. Futerman. Encephalopathy caused by ablation of very long acyl chain ceramide synthesis may be largely due to reduced galactosylceramide levels. J. Biol. Chem., 286(34):30022-33, aug 2011.

[63] T. Coetzee, N. Fujita, J. Dupree, R. Shi, A. Blight, K. Suzuki, and B. Popko. Myelination in the absence of galactocerebroside and sulfatide: normal structure with abnormal function and regional instability. Cell, 86(2):209-19, jul 1996.

[64] J. Marcus, S. Honigbaum, S. Shroff, K. Honke, J. Rosenbluth, and J. L. Dupree. Sulfatide is essential for the maintenance of CNS myelin and axon structure. Glia, 53(4):372-81, mar 2006.

[65] T. Hoshi, A. Suzuki, S. Hayashi, K. Tohyama, A. Hayashi, Y. Yamaguchi, K. Takeuchi, and H. Baba. Nodal protrusions, increased Schmidt-Lanterman incisures, and paranodal disorganization are characteristic features of sulfatide-deficient peripheral nerves. Glia, 55(6):584-94, apr 2007. 
[66] K. Suzuki. Globoid cell leukodystrophy (Krabbe's disease): update. J. Child Neurol., 18(9):595-603, sep 2003.

[67] V. Gieselmann. Metachromatic leukodystrophy: recent research developments. J. Child Neurol., 18(9):591-4, sep 2003.

[68] G. van Meer and Q. Lisman. Sphingolipid transport: rafts and translocators. J. Biol. Chem., 277(29):25855-8, jul 2002.

[69] J. B. Massey. Interaction of ceramides with phosphatidylcholine, sphingomyelin and sphingomyelin/cholesterol bilayers. Biochim. Biophys. Acta, 1510(1-2):167-84, feb 2001.

[70] A. Rietveld and K. Simons. The differential miscibility of lipids as the basis for the formation of functional membrane rafts. Biochim. Biophys. Acta, 1376(3):467-79, nov 1998.

[71] P. J. Espenshade and A. L. Hughes. Regulation of Sterol Synthesis in Eukaryotes. Annu. Rev. Genet., 41(1):401-427, dec 2007.

[72] G. J. Snipes and W. Orfali. Common themes in peripheral neuropathy disease genes. Cell Biol. Int., 22(11-12):815-35, nov 1998.

[73] I. Björkhem and S. Meaney. Brain cholesterol: long secret life behind a barrier. Arterioscler. Thromb. Vasc. Biol., 24(5):806-15, may 2004.

[74] D. Lingwood and K. Simons. Lipid rafts as a membrane-organizing principle. Science, 327(5961):46-50, jan 2010.

[75] J. M. Dietschy and S. D. Turley. Thematic review series: brain Lipids. Cholesterol metabolism in the central nervous system during early development and in the mature animal. J. Lipid Res., 45(8):1375-97, aug 2004.

[76] G. Saher, B. Brügger, C. Lappe-Siefke, W. Möbius, R.-i. Tozawa, M. C. Wehr, F. Wieland, S. Ishibashi, and K.-A. Nave. High cholesterol level is essential for myelin membrane growth. Nat. Neurosci., 8(4):468-75, apr 2005.

[77] G. Bu. Apolipoprotein E and its receptors in Alzheimer's disease: pathways, pathogenesis and therapy. Nat. Rev. Neurosci., 10(5):333-44, may 2009.

[78] G. J. Snipes and U. Suter. Cholesterol and myelin. Subcell. Biochem., 28:173-204, jan 1997.

[79] D. Boison and W. Stoffel. Disruption of the compacted myelin sheath of axons of the central nervous system in proteolipid protein-deficient mice. Proc. Natl. Acad. Sci. U. S. A., 91(24):11709-13, nov 1994.

[80] W. B. Macklin, C. W. Campagnoni, P. L. Deininger, and M. V. Gardinier. Structure and expression of the mouse myelin proteolipid protein gene. J. Neurosci. Res., 18(3):383-94, jan 1987.

[81] T. Weimbs and W. Stoffel. Proteolipid protein (PLP) of CNS myelin: positions of free, disulfide-bonded, and fatty acid thioester-linked cysteine residues and implications for the membrane topology of PLP. Biochemistry, 31(49):12289-96, dec 1992.

[82] M. Simons, E. M. Krämer, C. Thiele, W. Stoffel, and J. Trotter. Assembly of myelin by association of proteolipid protein with cholesterol- and galactosylceramide-rich membrane domains. J. Cell Biol., 151(1):143-54, oct 2000.

[83] K. A. Nave. Neurological mouse mutants and the genes of myelin. J. Neurosci. Res., 38(6):607-12, aug 1994.

[84] M. Klugmann, M. H. Schwab, A. Pühlhofer, A. Schneider, F. Zimmermann, I. R. Griffiths, and K. A. Nave. Assembly of CNS myelin in the absence of proteolipid protein. Neuron, 18(1):59-70, jan 1997. 
[85] D. A. Yool, J. M. Edgar, P. Montague, and S. Malcolm. The proteolipid protein gene and myelin disorders in man and animal models. Hum. Mol. Genet., 9(6):987-92, apr 2000.

[86] M. Simons, F. Schwärzler, D. Lütjohann, K. von Bergmann, K. Beyreuther, J. Dichgans, H. Wormstall, T. Hartmann, and J. B. Schulz. Treatment with simvastatin in normocholesterolemic patients with Alzheimer's disease: A 26-week randomized, placebo-controlled, double-blind trial. Ann. Neurol., 52(3):346-50, sep 2002.

[87] G. Saher, F. Rudolphi, K. Corthals, T. Ruhwedel, K.-F. Schmidt, S. Löwel, P. Dibaj, B. Barrette, W. Möbius, and K.-A. Nave. Therapy of Pelizaeus-Merzbacher disease in mice by feeding a cholesterol-enriched diet. Nat. Med., 18(7):1130-5, jul 2012.

[88] A. Roach, K. Boylan, S. Horvath, S. B. Prusiner, and L. E. Hood. Characterization of cloned cDNA representing rat myelin basic protein: absence of expression in brain of shiverer mutant mice. Cell, 34(3):799-806, oct 1983.

[89] J. M. Boggs, D. D. Wood, M. A. Moscarello, and D. Papahadjopoulos. Lipid phase separation induced by a hydrophobic protein in phosphatidylserinephosphatidylcholine vesicles. Biochemistry, 16(11):2325-9, may 1977.

[90] V. V. Bamm, M. De Avila, G. S. T. Smith, M. A. M. Ahmed, and G. Harauz. Structured functional domains of myelin basic protein: cross talk between actin polymerization and $\mathrm{Ca}(2+)$-dependent calmodulin interaction. Biophys. J., 101(5):1248-56, sep 2011.

[91] G. S. T. Smith, J. A. M. Voyer-Grant, and G. Harauz. Monitoring cleaved caspase-3 activity and apoptosis of immortalized oligodendroglial cells using live-cell imaging and cleaveable fluorogenic-dye substrates following potassium-induced membrane depolarization. J. Vis. Exp., (59), jan 2012.

[92] G. Harauz and D. S. Libich. The classic basic protein of myelin-conserved structural motifs and the dynamic molecular barcode involved in membrane adhesion and protein-protein interactions. Curr. Protein Pept. Sci., 10(3):196-215, jun 2009.

[93] V. Majava, E. Polverini, A. Mazzini, R. Nanekar, W. Knoll, J. Peters, F. Natali, P. Baumgärtel, I. Kursula, and P. Kursula. Structural and functional characterization of human peripheral nervous system myelin protein P2. PLoS One, 5(4):e10300, jan 2010 .

[94] D. S. Libich and G. Harauz. Backbone dynamics of the $18.5 \mathrm{kDa}$ isoform of myelin basic protein reveals transient alpha-helices and a calmodulin-binding site. Biophys. J., 94(12):4847-66, jun 2008.

[95] D. S. Libich, C. M. D. Hill, I. R. Bates, F. R. Hallett, S. Armstrong, A. Siemiarczuk, and G. Harauz. Interaction of the 18.5-kD isoform of myelin basic protein with $\mathrm{Ca} 2+$ -calmodulin: effects of deimination assessed by intrinsic Trp fluorescence spectroscopy, dynamic light scattering, and circular dichroism. Protein Sci., 12(7):1507-21, jul 2003.

[96] F. de Ferra, H. Engh, L. Hudson, J. Kamholz, C. Puckett, S. Molineaux, and R. A. Lazzarini. Alternative splicing accounts for the four forms of myelin basic protein. Cell, 43(3 Pt 2):721-7, dec 1985.

[97] J. Kamholz, J. Toffenetti, and R. A. Lazzarini. Organization and expression of the human myelin basic protein gene. J. Neurosci. Res., 21(1):62-70, sep 1988.

[98] J. Kamholz, R. Spielman, K. Gogolin, W. Modi, S. O'Brien, and R. Lazzarini. The human myelin-basic-protein gene: chromosomal localization and RFLP analysis. Am. J. Hum. Genet., 40(4):365-73, apr 1987.

[99] J. Kamholz, F. de Ferra, C. Puckett, and R. Lazzarini. Identification of three forms of human myelin basic protein by cDNA cloning. Proc. Natl. Acad. Sci. U. S. A., 83(13):4962-6, jul 1986. 
[100] A. T. Campagnoni, T. M. Pribyl, C. W. Campagnoni, K. Kampf, S. Amur-Umarjee, C. F. Landry, V. W. Handley, S. L. Newman, B. Garbay, and K. Kitamura. Structure and developmental regulation of Golli-mbp, a 105-kilobase gene that encompasses the myelin basic protein gene and is expressed in cells in the oligodendrocyte lineage in the brain. J. Biol. Chem., 268(7):4930-8, mar 1993.

[101] P. M. Paez, V. Spreuer, V. Handley, J.-M. Feng, C. Campagnoni, and A. T. Campagnoni. Increased expression of golli myelin basic proteins enhances calcium influx into oligodendroglial cells. J. Neurosci., 27(46):12690-9, nov 2007.

[102] C. F. Landry, J. Ellison, E. Skinner, and A. T. Campagnoni. Golli-MBP proteins mark the earliest stages of fiber extension and terminal arboration in the mouse peripheral nervous system. J. Neurosci. Res., 50(2):265-71, oct 1997.

[103] T. M. Pribyl, C. W. Campagnoni, K. Kampf, T. Kashima, V. W. Handley, J. McMahon, and A. T. Campagnoni. The human myelin basic protein gene is included within a 179-kilobase transcription unit: expression in the immune and central nervous systems. Proc. Natl. Acad. Sci. U. S. A., 90(22):10695-9, nov 1993.

[104] H. J. Roth, K. E. Kronquist, N. Kerlero de Rosbo, B. F. Crandall, and A. T. Campagnoni. Evidence for the expression of four myelin basic protein variants in the developing human spinal cord through cDNA cloning. J. Neurosci. Res., 17(4):321-8, jan 1987.

[105] E. Barbarese, P. E. Braun, and J. H. Carson. Identification of prelarge and presmall basic proteins in mouse myelin and their structural relationship to large and small basic proteins. Proc. Natl. Acad. Sci. U. S. A., 74(8):3360-4, aug 1977.

[106] L. Pedraza. Nuclear transport of myelin basic protein. J. Neurosci. Res., 50(2):25864, oct 1997.

[107] D. R. Colman, G. Kreibich, A. B. Frey, and D. D. Sabatini. Synthesis and incorporation of myelin polypeptides into CNS myelin. J. Cell Biol., 95(2 Pt 1):598-608, nov 1982.

[108] K. Kristensson, N. K. Zeller, M. E. Dubois-Dalcq, and R. A. Lazzarini. Expression of myelin basic protein gene in the developing rat brain as revealed by in situ hybridization. J. Histochem. Cytochem., 34(4):467-73, apr 1986.

[109] B. D. Trapp, T. Moench, M. Pulley, E. Barbosa, G. Tennekoon, and J. Griffin. Spatial segregation of mRNA encoding myelin-specific proteins. Proc. Natl. Acad. Sci. U. S. A., 84(21):7773-7, nov 1987.

[110] K. Ainger, D. Avossa, F. Morgan, S. J. Hill, C. Barry, E. Barbarese, and J. H. Carson. Transport and localization of exogenous myelin basic protein mRNA microinjected into oligodendrocytes. J. Cell Biol., 123(2):431-41, oct 1993.

[111] K. Ainger, D. Avossa, A. S. Diana, C. Barry, E. Barbarese, and J. H. Carson. Transport and localization elements in myelin basic protein mRNA. J. Cell Biol., 138(5):1077-87, sep 1997.

[112] R. White, C. Gonsior, E.-M. Krämer-Albers, N. Stöhr, S. Hüttelmaier, and J. Trotter. Activation of oligodendroglial Fyn kinase enhances translation of mRNAs transported in hnRNP A2-dependent RNA granules. J. Cell Biol., 181(4):579-86, may 2008.

[113] S. S. Zamvil, D. J. Mitchell, A. C. Moore, K. Kitamura, L. Steinman, and J. B. Rothbard. T-cell epitope of the autoantigen myelin basic protein that induces encephalomyelitis. Nature, 324(6094):258-60, jan 1986.

[114] L. Cao, R. Goodin, D. Wood, M. A. Moscarello, and J. N. Whitaker. Rapid release and unusual stability of immunodominant peptide 45-89 from citrullinated myelin basic protein. Biochemistry, 38(19):6157-63, may 1999. 
[115] T. Vartanian, S. Szuchet, G. Dawson, and A. T. Campagnoni. Oligodendrocyte adhesion activates protein kinase C-mediated phosphorylation of myelin basic protein. Science, 234(4782):1395-8, dec 1986.

[116] C. M. D. Hill and G. Harauz. Charge effects modulate actin assembly by classic myelin basic protein isoforms. Biochem. Biophys. Res. Commun., 329(1):362-9, apr 2005 .

[117] J. M. Boggs, G. Rangaraj, W. Gao, and Y.-M. Heng. Effect of phosphorylation of myelin basic protein by MAPK on its interactions with actin and actin binding to a lipid membrane in vitro. Biochemistry, 45(2):391-401, jan 2006.

[118] J. B. Ulmer and P. E. Braun. In vivo phosphorylation of myelin basic proteins in developing mouse brain: evidence that phosphorylation is an early event in myelin formation. Dev. Neurosci., 6(6):345-55, jan 1983.

[119] J. K. Kim, F. G. Mastronardi, D. D. Wood, D. M. Lubman, R. Zand, and M. a. Moscarello. Multiple sclerosis: an important role for post-translational modifications of myelin basic protein in pathogenesis. Mol. Cell. Proteomics, 2(7):453-62, jul 2003.

[120] L. S. DeBruin, J. D. Haines, L. A. Wellhauser, G. Radeva, V. Schonmann, D. Bienzle, and G. Harauz. Developmental partitioning of myelin basic protein into membrane microdomains. J. Neurosci. Res., 80(2):211-25, apr 2005.

[121] L. S. DeBruin, J. D. Haines, D. Bienzle, and G. Harauz. Partitioning of myelin basic protein into membrane microdomains in a spontaneously demyelinating mouse model for multiple sclerosis. Biochem. Cell Biol., 84(6):993-1005, dec 2006.

[122] L. S. Debruin and G. Harauz. White matter rafting-membrane microdomains in myelin. Neurochem. Res., 32(2):213-28, feb 2007.

[123] J. J. Ramwani, R. M. Epand, and M. A. Moscarello. Secondary structure of charge isomers of myelin basic protein before and after phosphorylation. Biochemistry, 28(16):6538-43, aug 1989.

[124] J. M. Boggs, P. M. Yip, G. Rangaraj, and E. Jo. Effect of posttranslational modifications to myelin basic protein on its ability to aggregate acidic lipid vesicles. Biochemistry, 36(16):5065-71, apr 1997.

[125] D. R. Beniac, D. D. Wood, N. Palaniyar, F. P. Ottensmeyer, M. A. Moscarello, and G. Harauz. Cryoelectron microscopy of protein-lipid complexes of human myelin basic protein charge isomers differing in degree of citrullination. J. Struct. Biol., 129(1):80-95, feb 2000.

[126] G. W. Brady, D. B. Fein, D. D. Wood, and M. A. Moscarello. The role of charge microheterogeneity of human myelin basic protein in the formation of phosphatidylglycerol multilayers. Biochem. Biophys. Res. Commun., 126(3):1161-5, feb 1985.

[127] L. B. Pritzker, S. Joshi, J. J. Gowan, G. Harauz, and M. A. Moscarello. Deimination of myelin basic protein. 1. Effect of deimination of arginyl residues of myelin basic protein on its structure and susceptibility to digestion by cathepsin D. Biochemistry, 39(18):5374-81, may 2000.

[128] A. A. Musse, J. M. Boggs, and G. Harauz. Deimination of membrane-bound myelin basic protein in multiple sclerosis exposes an immunodominant epitope. Proc. Natl. Acad. Sci. U. S. A., 103(12):4422-7, mar 2006.

[129] E. R. Einstein, J. Csejtey, K. B. Dalal, C. W. Adams, O. B. Bayliss, and J. F. Hallpike. Proteolytic activity and basic protein loss in and around multiple sclerosis plaques: combined biochemical and histochemical observations. J. Neurochem., 19(3):653-62, mar 1972.

[130] M. L. Cuzner and A. N. Davison. Changes in cerebral lysosomal enzyme activity and lipids in multiple sclerosis. J. Neurol. Sci., 19(1):29-36, may 1973. 
[131] G. Harauz and A. A. Musse. A tale of two citrullines-structural and functional aspects of myelin basic protein deimination in health and disease. Neurochem. Res., 32(2):137-58, feb 2007.

[132] M. A. Moscarello, F. G. Mastronardi, and D. D. Wood. The role of citrullinated proteins suggests a novel mechanism in the pathogenesis of multiple sclerosis. Neurochem. Res., 32(2):251-6, feb 2007.

[133] D. D. Wood, C. A. Ackerley, B. van den Brand, L. Zhang, R. Raijmakers, F. G. Mastronardi, and M. A. Moscarello. Myelin localization of peptidylarginine deiminases 2 and 4: comparison of PAD2 and PAD4 activities. Lab. Invest., 88(4):354-64, apr 2008.

[134] C. M. Bradford, I. Ramos, A. K. Cross, G. Haddock, S. McQuaid, A. P. Nicholas, and M. N. Woodroofe. Localisation of citrullinated proteins in normal appearing white matter and lesions in the central nervous system in multiple sclerosis. $J$. Neuroimmunol., 273(1-2):85-95, aug 2014.

[135] M. A. Moscarello, D. D. Wood, C. Ackerley, and C. Boulias. Myelin in multiple sclerosis is developmentally immature. J. Clin. Invest., 94(1):146-54, jul 1994.

[136] D. D. Wood and M. A. Moscarello. The isolation, characterization, and lipidaggregating properties of a citrulline containing myelin basic protein. J. Biol. Chem., 264(9):5121-7, mar 1989.

[137] J. M. Boggs, G. Rangaraj, K. M. Koshy, C. Ackerley, D. D. Wood, and M. A. Moscarello. Highly deiminated isoform of myelin basic protein from multiple sclerosis brain causes fragmentation of lipid vesicles. J. Neurosci. Res., 57(4):529-35, aug 1999.

[138] Y. Hu, I. Doudevski, D. Wood, M. Moscarello, C. Husted, C. Genain, J. A. Zasadzinski, and J. Israelachvili. Synergistic interactions of lipids and myelin basic protein. Proc. Natl. Acad. Sci. U. S. A., 101(37):13466-71, sep 2004.

[139] H.-a. Ro and J. H. Carson. pH microdomains in oligodendrocytes. J. Biol. Chem., 279(35):37115-23, aug 2004.

[140] E. Jo and J. M. Boggs. Aggregation of acidic lipid vesicles by myelin basic protein: dependence on potassium concentration. Biochemistry, 34(41):13705-16, oct 1995.

[141] D. Marsh, L. I. Horváth, M. J. Swamy, S. Mantripragada, and J. H. Kleinschmidt. Interaction of membrane-spanning proteins with peripheral and lipid-anchored membrane proteins: perspectives from protein-lipid interactions (Review). Mol. Membr. Biol., 19(4):247-55, jan 2002.

[142] D. Fitzner, A. Schneider, A. Kippert, W. Möbius, K. I. Willig, S. W. Hell, G. Bunt, K. Gaus, and M. Simons. Myelin basic protein-dependent plasma membrane reorganization in the formation of myelin. EMBO J., 25(21):5037-48, nov 2006.

[143] C. M. Rosetti and B. Maggio. Protein-induced surface structuring in myelin membrane monolayers. Biophys. J., 93(12):4254-67, dec 2007.

[144] Y. Hu and J. Israelachvili. Lateral reorganization of myelin lipid domains by myelin basic protein studied at the air-water interface. Colloids Surf. B. Biointerfaces, 62(1):22-30, mar 2008.

[145] C. A. Dyer and J. A. Benjamins. Organization of oligodendroglial membrane sheets: II. Galactocerebroside:antibody interactions signal changes in cytoskeleton and myelin basic protein. J. Neurosci. Res., 24(2):212-21, oct 1989.

[146] C. A. Dyer, T. M. Philibotte, M. K. Wolf, and S. Billings-Gagliardi. Myelin basic protein mediates extracellular signals that regulate microtubule stability in oligodendrocyte membrane sheets. J. Neurosci. Res., 39(1):97-107, sep 1994. 
[147] J. M. Boggs, W. Gao, and Y. Hirahara. Signal transduction pathways involved in interaction of galactosylceramide/sulfatide-containing liposomes with cultured oligodendrocytes and requirement for myelin basic protein and glycosphingolipids. J. Neurosci. Res., 86(7):1448-58, may 2008.

[148] C. M. Hill, I. R. Bates, G. F. White, F. R. Hallett, and G. Harauz. Effects of the osmolyte trimethylamine-N-oxide on conformation, self-association, and twodimensional crystallization of myelin basic protein. J. Struct. Biol., 139(1):13-26, jul 2002.

[149] P. Romero, Z. Obradovic, X. Li, E. C. Garner, C. J. Brown, and A. K. Dunker. Sequence complexity of disordered protein. Proteins, 42(1):38-48, jan 2001.

[150] P. Tompa. Intrinsically unstructured proteins. Trends Biochem. Sci., 27(10):527-33, oct 2002 .

[151] M. A. Keniry and R. Smith. Circular dichroic analysis of the secondary structure of myelin basic protein and derived peptides bound to detergents and to lipid vesicles. Biochim. Biophys. Acta, 578(2):381-91, jun 1979.

[152] E. Polverini, A. Fasano, F. Zito, P. Riccio, and P. Cavatorta. Conformation of bovine myelin basic protein purified with bound lipids. Eur. Biophys. J., 28(4):351-5, jan 1999.

[153] R. A. Ridsdale, D. R. Beniac, T. A. Tompkins, M. A. Moscarello, and G. Harauz. Three-dimensional structure of myelin basic protein. II. Molecular modeling and considerations of predicted structures in multiple sclerosis. J. Biol. Chem., 272(7):4269-75, feb 1997.

[154] D. W. Lee, X. Banquy, K. Kristiansen, Y. Min, A. Ramachandran, J. M. Boggs, and J. N. Israelachvili. Adsorption Mechanism of Myelin Basic Protein on Model Substrates and Its Bridging Interaction between the Two Surfaces. Langmuir, 31(10):3159-66, mar 2015.

[155] K. A. Vassall, A. D. Jenkins, V. V. Bamm, and G. Harauz. Thermodynamic Analysis of the Disorder-to- $\alpha$-Helical Transition of $18.5-\mathrm{kDa}$ Myelin Basic Protein Reveals an Equilibrium Intermediate Representing the Most Compact Conformation. J. Mol. Biol., 427(10):1977-92, may 2015.

[156] C. Bakolitsa, J. M. de Pereda, C. R. Bagshaw, D. R. Critchley, and R. C. Liddington. Crystal structure of the vinculin tail suggests a pathway for activation. Cell, 99(6):603-13, dec 1999.

[157] K. W. Wucherpfennig, J. Zhang, C. Witek, M. Matsui, Y. Modabber, K. Ota, and D. A. Hafler. Clonal expansion and persistence of human $\mathrm{T}$ cells specific for an immunodominant myelin basic protein peptide. J. Immunol., 152(11):5581-92, jun 1994.

[158] R. Martin, M. D. Howell, D. Jaraquemada, M. Flerlage, J. Richert, S. Brostoff, E. O. Long, D. E. McFarlin, and H. F. McFarland. A myelin basic protein peptide is recognized by cytotoxic $\mathrm{T}$ cells in the context of four HLA-DR types associated with multiple sclerosis. J. Exp. Med., 173(1):19-24, jan 1991.

[159] C. Farès, D. S. Libich, and G. Harauz. Solution NMR structure of an immunodominant epitope of myelin basic protein. Conformational dependence on environment of an intrinsically unstructured protein. FEBS J., 273(3):601-14, feb 2006.

[160] I. R. Bates, J. B. Feix, J. M. Boggs, and G. Harauz. An immunodominant epitope of myelin basic protein is an amphipathic alpha-helix. J. Biol. Chem., 279(7):5757-64, feb 2004.

[161] E. Strandberg and J. A. Killian. Snorkeling of lysine side chains in transmembrane helices: how easy can it get? FEBS Lett., 544(1-3):69-73, jun 2003. 
[162] I. R. Bates, J. M. Boggs, J. B. Feix, and G. Harauz. Membrane-anchoring and charge effects in the interaction of myelin basic protein with lipid bilayers studied by site-directed spin labeling. J. Biol. Chem., 278(31):29041-7, aug 2003.

[163] K. A. Vassall, K. Bessonov, M. De Avila, E. Polverini, and G. Harauz. The effects of threonine phosphorylation on the stability and dynamics of the central molecular switch region of 18.5-kDa myelin basic protein. PLoS One, 8(7):e68175, jan 2013.

[164] D. R. Beniac, M. D. Luckevich, G. J. Czarnota, T. A. Tompkins, R. A. Ridsdale, F. P. Ottensmeyer, M. A. Moscarello, and G. Harauz. Three-dimensional structure of myelin basic protein. I. Reconstruction via angular reconstitution of randomly oriented single particles. J. Biol. Chem., 272(7):4261-8, feb 1997.

[165] G. F. Chernoff. Shiverer: an autosomal recessive mutant mouse with myelin deficiency. J. Hered., 72(2):128, jan.

[166] A. Privat, C. Jacque, J. M. Bourre, P. Dupouey, and N. Baumann. Absence of the major dense line in myelin of the mutant mouse "shiverer". Neurosci. Lett., 12(1):107-12, apr 1979.

[167] C. Readhead and L. Hood. The dysmyelinating mouse mutations shiverer (shi) and myelin deficient (shimld). Behav. Genet., 20(2):213-34, mar 1990.

[168] X. Y. Shen, S. Billings-Gagliardi, R. L. Sidman, and M. K. Wolf. Myelin deficient (shimld) mutant allele: morphological comparison with shiverer (shi) allele on a B6C3 mouse stock. Brain Res., 360(1-2):235-47, dec 1985.

[169] C. A. Dyer, T. M. Philibotte, S. Billings-Gagliardi, and M. K. Wolf. Cytoskeleton in myelin-basic-protein-deficient shiverer oligodendrocytes. Dev. Neurosci., 17(1):5362, jan 1995.

[170] C. Readhead, B. Popko, N. Takahashi, H. D. Shine, R. A. Saavedra, R. L. Sidman, and L. Hood. Expression of a myelin basic protein gene in transgenic shiverer mice: correction of the dysmyelinating phenotype. Cell, 48(4):703-12, feb 1987.

[171] M. Kimura, M. Sato, A. Akatsuka, S. Nozawa-Kimura, R. Takahashi, M. Yokoyama, T. Nomura, and M. Katsuki. Restoration of myelin formation by a single type of myelin basic protein in transgenic shiverer mice. Proc. Natl. Acad. Sci. U. S. A., 86(14):5661-5, jul 1989.

[172] M. Katsuki, M. Sato, M. Kimura, M. Yokoyama, K. Kobayashi, and T. Nomura. Conversion of normal behavior to shiverer by myelin basic protein antisense cDNA in transgenic mice. Science, 241(4865):593-5, jul 1988.

[173] H. Okano, M. Miura, A. Moriguchi, K. Ikenaka, Y. Tsukada, and K. Mikoshiba. Inefficient transcription of the myelin basic protein gene possibly causes hypomyelination in myelin-deficient mutant mice. J. Neurochem., 48(2):470-6, feb 1987.

[174] A. A. Akowitz, E. Barbarese, K. Scheld, and J. H. Carson. Structure and expression of myelin basic protein gene sequences in the mld mutant mouse: reiteration and rearrangement of the MBP gene. Genetics, 116(3):447-64, jul 1987.

[175] A. Iglesias, J. Bauer, T. Litzenburger, A. Schubart, and C. Linington. T- and Bcell responses to myelin oligodendrocyte glycoprotein in experimental autoimmune encephalomyelitis and multiple sclerosis. Glia, 36(2):220-34, nov 2001.

[176] J. F. Kroepfl, L. R. Viise, A. J. Charron, C. Linington, and M. V. Gardinier. Investigation of myelin/oligodendrocyte glycoprotein membrane topology. J. Neurochem., 67(5):2219-22, nov 1996.

[177] N. J. Scolding, S. Frith, C. Linington, B. P. Morgan, A. K. Campbell, and D. A. Compston. Myelin-oligodendrocyte glycoprotein (MOG) is a surface marker of oligodendrocyte maturation. J. Neuroimmunol., 22(3):169-76, may 1989. 
[178] T. Berger and M. Reindl. Immunopathogenic and clinical relevance of antibodies against myelin oligodendrocyte glycoprotein (MOG) in Multiple Sclerosis. J. Neural Transm. Suppl., (60):351-60, jan 2000.

[179] T. G. Johns and C. C. Bernard. The structure and function of myelin oligodendrocyte glycoprotein. J. Neurochem., 72(1):1-9, jan 1999.

[180] C. Delarasse, P. Daubas, L. T. Mars, C. Vizler, T. Litzenburger, A. Iglesias, J. Bauer, B. Della Gaspera, A. Schubart, L. Decker, D. Dimitri, G. Roussel, A. Dierich, S. Amor, A. Dautigny, R. Liblau, and D. Pham-Dinh. Myelin/oligodendrocyte glycoprotein-deficient (MOG-deficient) mice reveal lack of immune tolerance to MOG in wild-type mice. J. Clin. Invest., 112(4):544-53, aug 2003.

[181] M. M. Morris-Downes, P. A. Smith, J. L. Rundle, S. J. Piddlesden, D. Baker, D. Pham-Dinh, N. Heijmans, and S. Amor. Pathological and regulatory effects of anti-myelin antibodies in experimental allergic encephalomyelitis in mice. $J$. Neuroimmunol., 125(1-2):114-24, apr 2002.

[182] K. L. de Graaf, M. Albert, and R. Weissert. Autoantigen conformation influences both B- and T-cell responses and encephalitogenicity. J. Biol. Chem., 287(21):17206-13, may 2012.

[183] P. H. Lalive. Autoantibodies in inflammatory demyelinating diseases of the central nervous system. Swiss Med. Wkly., 138(47-48):692-707, nov 2008.

[184] S. Saadoun, P. Waters, G. P. Owens, J. L. Bennett, A. Vincent, and M. C. Papadopoulos. Neuromyelitis optica MOG-IgG causes reversible lesions in mouse brain. Acta Neuropathol. Commun., 2:35, jan 2014.

[185] N. H. Sternberger, R. H. Quarles, Y. Itoyama, and H. D. Webster. Myelin-associated glycoprotein demonstrated immunocytochemically in myelin and myelin-forming cells of developing rat. Proc. Natl. Acad. Sci. U. S. A., 76(3):1510-4, mar 1979.

[186] B. D. Trapp and R. H. Quarles. Presence of the myelin-associated glycoprotein correlates with alterations in the periodicity of peripheral myelin. J. Cell Biol., 92(3):877-82, mar 1982.

[187] D. Burger, G. Perruisseau, M. Simon, and A. J. Steck. Comparison of the N-linked oligosaccharide structures of the two major human myelin glycoproteins MAG and P0: assessment and relative occurrence of oligosaccharide structures by serial lectin affinity chromatography of 14C-glycopeptides. J. Neurochem., 58(3):845-53, mar 1992.

[188] A. Varki and T. Angata. Siglecs-the major subfamily of I-type lectins. Glycobiology, 16(1):1R-27R, jan 2006.

[189] P. Kursula. The current status of structural studies on proteins of the myelin sheath (Review). Int. J. Mol. Med., 8(5):475-9, nov 2001.

[190] P. Kursula, V. P. Lehto, and A. M. Heape. S100beta inhibits the phosphorylation of the L-MAG cytoplasmic domain by PKA. Brain Res. Mol. Brain Res., 76(2):407-10, mar 2000 .

[191] M. L. Jaramillo, D. E. Afar, G. Almazan, and J. C. Bell. Identification of tyrosine 620 as the major phosphorylation site of myelin-associated glycoprotein and its implication in interacting with signaling molecules. J. Biol. Chem., 269(44):272405, nov 1994.

[192] D. E. Afar, J. L. Salzer, J. Roder, P. E. Braun, and J. C. Bell. Differential phosphorylation of myelin-associated glycoprotein isoforms in cell culture. J. Neurochem., 55(4):1418-26, oct 1990.

[193] R. L. Schnaar, B. E. Collins, L. P. Wright, M. Kiso, M. B. Tropak, J. C. Roder, and P. R. Crocker. Myelin-associated glycoprotein binding to gangliosides. Structural specificity and functional implications. Ann. N. Y. Acad. Sci., 845:92-105, jun 1998. 
[194] H. Umemori, S. Sato, T. Yagi, S. Aizawa, and T. Yamamoto. Initial events of myelination involve Fyn tyrosine kinase signalling. Nature, 367(6463):572-6, feb 1994.

[195] D. Montag, K. P. Giese, U. Bartsch, R. Martini, Y. Lang, H. Blüthmann, J. Karthigasan, D. A. Kirschner, E. S. Wintergerst, and K. A. Nave. Mice deficient for the myelin-associated glycoprotein show subtle abnormalities in myelin. Neuron, 13(1):229-46, jul 1994.

[196] C. Li, M. B. Tropak, R. Gerlai, S. Clapoff, W. Abramow-Newerly, B. Trapp, A. Peterson, and J. Roder. Myelination in the absence of myelin-associated glycoprotein. Nature, 369(6483):747-50, jun 1994.

[197] U. S. Vogel, R. Reynolds, R. J. Thompson, and G. P. Wilkin. Expression of the 2',3'-cyclic nucleotide 3'-phosphohydrolase gene and immunoreactive protein in oligodendrocytes as revealed by in situ hybridization and immunofluorescence. Glia, 1(3):184-90, jan 1988.

[198] R. C. O'Neill, J. Minuk, M. E. Cox, P. E. Braun, and M. Gravel. CNP2 mRNA directs synthesis of both CNP1 and CNP2 polypeptides. J. Neurosci. Res., 50(2):24857, oct 1997.

[199] P. E. Braun, D. De Angelis, W. W. Shtybel, and L. Bernier. Isoprenoid modification permits 2',3'-cyclic nucleotide 3'-phosphodiesterase to bind to membranes. $J$. Neurosci. Res., 30(3):540-4, nov 1991.

[200] J. Lee, M. Gravel, R. Zhang, P. Thibault, and P. E. Braun. Process outgrowth in oligodendrocytes is mediated by CNP, a novel microtubule assembly myelin protein. J. Cell Biol., 170(4):661-73, aug 2005.

[201] B. F. G. Popescu and C. F. Lucchinetti. Pathology of demyelinating diseases. Annu. Rev. Pathol., 7:185-217, jan 2012.

[202] S. L. Hauser and J. R. Oksenberg. The neurobiology of multiple sclerosis: genes, inflammation, and neurodegeneration. Neuron, 52(1):61-76, oct 2006.

[203] C. Confavreux, G. Aimard, and M. Devic. Course and prognosis of multiple sclerosis assessed by the computerized data processing of 349 patients. Brain, 103(2):281-300, jun 1980 .

[204] J. H. Noseworthy, C. Lucchinetti, M. Rodriguez, and B. G. Weinshenker. Multiple sclerosis. N. Engl. J. Med., 343(13):938-52, sep 2000.

[205] C. Confavreux, S. Vukusic, T. Moreau, and P. Adeleine. Relapses and progression of disability in multiple sclerosis. N. Engl. J. Med., 343(20):1430-8, nov 2000.

[206] J. Antel, S. Antel, Z. Caramanos, D. L. Arnold, and T. Kuhlmann. Primary progressive multiple sclerosis: part of the MS disease spectrum or separate disease entity? Acta Neuropathol., 123(5):627-38, may 2012.

[207] B. D. Trapp, J. Peterson, R. M. Ransohoff, R. Rudick, S. Mörk, and L. Bö. Axonal transection in the lesions of multiple sclerosis. N. Engl. J. Med., 338(5):278-85, jan 1998.

[208] A. Bitsch, J. Schuchardt, S. Bunkowski, T. Kuhlmann, and W. Brück. Acute axonal injury in multiple sclerosis. Correlation with demyelination and inflammation. Brain, 123 ( Pt 6:1174-83, jun 2000.

[209] B. Ferguson, M. K. Matyszak, M. M. Esiri, and V. H. Perry. Axonal damage in acute multiple sclerosis lesions. Brain, 120 ( Pt 3:393-9, mar 1997.

[210] P. van der Valk and C. J. De Groot. Staging of multiple sclerosis (MS) lesions: pathology of the time frame of MS. Neuropathol. Appl. Neurobiol., 26(1):2-10, feb 2000 . 
[211] M. Kipp, T. Clarner, J. Dang, S. Copray, and C. Beyer. The cuprizone animal model: new insights into an old story. Acta Neuropathol., 118(6):723-36, dec 2009.

[212] H. Lassmann. Mechanisms of inflammation induced tissue injury in multiple sclerosis. J. Neurol. Sci., 274(1-2):45-7, nov 2008.

[213] C. Lucchinetti, W. Brück, J. Parisi, B. Scheithauer, M. Rodriguez, and H. Lassmann. Heterogeneity of multiple sclerosis lesions: implications for the pathogenesis of demyelination. Ann. Neurol., 47(6):707-17, jun 2000.

[214] S. Cepok, M. Jacobsen, S. Schock, B. Omer, S. Jaekel, I. Böddeker, W. H. Oertel, N. Sommer, and B. Hemmer. Patterns of cerebrospinal fluid pathology correlate with disease progression in multiple sclerosis. Brain, 124(Pt 11):2169-76, nov 2001.

[215] A. Kutzelnigg, J. C. Faber-Rod, J. Bauer, C. F. Lucchinetti, P. S. Sorensen, H. Laursen, C. Stadelmann, W. Brück, H. Rauschka, M. Schmidbauer, and H. Lassmann. Widespread demyelination in the cerebellar cortex in multiple sclerosis. Brain Pathol., 17(1):38-44, jan 2007.

[216] C. A. Husted, G. B. Matson, D. A. Adams, D. S. Goodin, and M. W. Weiner. In vivo detection of myelin phospholipids in multiple sclerosis with phosphorus magnetic resonance spectroscopic imaging. Ann. Neurol., 36(2):239-41, aug 1994.

[217] D. Kidd, F. Barkhof, R. McConnell, P. R. Algra, I. V. Allen, and T. Revesz. Cortical lesions in multiple sclerosis. Brain, 122 ( Pt 1:17-26, jan 1999.

[218] J. W. Peterson, L. Bö, S. Mörk, A. Chang, and B. D. Trapp. Transected neurites, apoptotic neurons, and reduced inflammation in cortical multiple sclerosis lesions. Ann. Neurol., 50(3):389-400, sep 2001.

[219] O. Périer and A. Grégoire. Electron microscopic features of multiple sclerosis lesions. Brain, 88(5):937-52, dec 1965.

[220] P. K. Stys. Multiple sclerosis: autoimmune disease or autoimmune reaction? Can. J. Neurol. Sci., 37 Suppl 2:S16-23, sep 2010.

[221] Y. K. Chou, D. N. Bourdette, H. Offner, R. Whitham, R. Y. Wang, G. A. Hashim, and A. A. Vandenbark. Frequency of T cells specific for myelin basic protein and myelin proteolipid protein in blood and cerebrospinal fluid in multiple sclerosis. $J$. Neuroimmunol., 38(1-2):105-13, may 1992.

[222] J. Zhang, S. Markovic-Plese, B. Lacet, J. Raus, H. L. Weiner, and D. A. Hafler. Increased frequency of interleukin 2-responsive $\mathrm{T}$ cells specific for myelin basic protein and proteolipid protein in peripheral blood and cerebrospinal fluid of patients with multiple sclerosis. J. Exp. Med., 179(3):973-84, mar 1994.

[223] A. E. Lovett-Racke, J. L. Trotter, J. Lauber, P. J. Perrin, C. H. June, and M. K. Racke. Decreased dependence of myelin basic protein-reactive T cells on CD28mediated costimulation in multiple sclerosis patients. A marker of activated/memory T cells. J. Clin. Invest., 101(4):725-30, feb 1998.

[224] N. Hellings, M. Barée, C. Verhoeven, M. B. D’hooghe, R. Medaer, C. C. Bernard, J. Raus, and P. Stinissen. T-cell reactivity to multiple myelin antigens in multiple sclerosis patients and healthy controls. J. Neurosci. Res., 63(3):290-302, feb 2001.

[225] M. Jacobsen, S. Cepok, E. Quak, M. Happel, R. Gaber, A. Ziegler, S. Schock, W. H. Oertel, N. Sommer, and B. Hemmer. Oligoclonal expansion of memory CD8+ T cells in cerebrospinal fluid from multiple sclerosis patients. Brain, $125(\mathrm{Pt} 3): 538-50$, mar 2002.

[226] H. Babbe, A. Roers, A. Waisman, H. Lassmann, N. Goebels, R. Hohlfeld, M. Friese, R. Schröder, M. Deckert, S. Schmidt, R. Ravid, and K. Rajewsky. Clonal expansions of CD8(+) $\mathrm{T}$ cells dominate the $\mathrm{T}$ cell infiltrate in active multiple sclerosis lesions as shown by micromanipulation and single cell polymerase chain reaction. J. Exp. Med., 192(3):393-404, aug 2000. 
[227] A. Jurewicz, W. E. Biddison, and J. P. Antel. MHC class I-restricted lysis of human oligodendrocytes by myelin basic protein peptide-specific CD8 T lymphocytes. $J$. Immunol., 160(6):3056-9, mar 1998.

[228] I. Medana, M. A. Martinic, H. Wekerle, and H. Neumann. Transection of major histocompatibility complex class I-induced neurites by cytotoxic $\mathrm{T}$ lymphocytes. Am. J. Pathol., 159(3):809-15, sep 2001.

[229] R. Höftberger, F. Aboul-Enein, W. Brueck, C. Lucchinetti, M. Rodriguez, M. Schmidbauer, K. Jellinger, and H. Lassmann. Expression of major histocompatibility complex class I molecules on the different cell types in multiple sclerosis lesions. Brain Pathol., 14(1):43-50, jan 2004.

[230] D. Sun, J. N. Whitaker, Z. Huang, D. Liu, C. Coleclough, H. Wekerle, and C. S. Raine. Myelin antigen-specific CD8+ T cells are encephalitogenic and produce severe disease in C57BL/6 mice. J. Immunol., 166(12):7579-87, jun 2001.

[231] R. Martin, H. F. McFarland, and D. E. McFarlin. Immunological aspects of demyelinating diseases. Annu. Rev. Immunol., 10:153-87, jan 1992.

[232] M. Sospedra and R. Martin. Immunology of multiple sclerosis. Annu. Rev. Immunol., 23:683-747, jan 2005.

[233] H. Wiendl and R. Hohlfeld. Therapeutic approaches in multiple sclerosis: lessons from failed and interrupted treatment trials. BioDrugs, 16(3):183-200, jan 2002.

[234] M. E. Smith. Phagocytic properties of microglia in vitro: implications for a role in multiple sclerosis and EAE. Microsc. Res. Tech., 54(2):81-94, jul 2001.

[235] K. J. Smith, R. Kapoor, and P. A. Felts. Demyelination: the role of reactive oxygen and nitrogen species. Brain Pathol., 9(1):69-92, jan 1999.

[236] M. Kipp, S. Karakaya, S. Johann, E. Kampmann, J. Mey, and C. Beyer. Oestrogen and progesterone reduce lipopolysaccharide-induced expression of tumour necrosis factor-alpha and interleukin-18 in midbrain astrocytes. J. Neuroendocrinol., 19(10):819-22, oct 2007.

[237] M. Kipp, A. Norkute, S. Johann, L. Lorenz, A. Braun, A. Hieble, S. Gingele, F. Pott, J. Richter, and C. Beyer. Brain-region-specific astroglial responses in vitro after LPS exposure. J. Mol. Neurosci., 35(2):235-43, jun 2008.

[238] S. A. Back, T. M. F. Tuohy, H. Chen, N. Wallingford, A. Craig, J. Struve, N. L. Luo, F. Banine, Y. Liu, A. Chang, B. D. Trapp, B. F. Bebo, M. S. Rao, and L. S. Sherman. Hyaluronan accumulates in demyelinated lesions and inhibits oligodendrocyte progenitor maturation. Nat. Med., 11(9):966-72, sep 2005.

[239] C. L. Vanderlugt and S. D. Miller. Epitope spreading in immune-mediated diseases: implications for immunotherapy. Nat. Rev. Immunol., 2(2):85-95, feb 2002.

[240] A. M. Carrizosa, L. B. Nicholson, M. Farzan, S. Southwood, A. Sette, R. A. Sobel, and V. K. Kuchroo. Expansion by self antigen is necessary for the induction of experimental autoimmune encephalomyelitis by $\mathrm{T}$ cells primed with a cross-reactive environmental antigen. J. Immunol., 161(7):3307-14, oct 1998.

[241] K. W. Wucherpfennig and J. L. Strominger. Molecular mimicry in T cell-mediated autoimmunity: viral peptides activate human $\mathrm{T}$ cell clones specific for myelin basic protein. Cell, 80(5):695-705, mar 1995.

[242] R. S. Fujinami and M. B. Oldstone. Amino acid homology between the encephalitogenic site of myelin basic protein and virus: mechanism for autoimmunity. Science, 230(4729):1043-5, nov 1985.

[243] G. Martino, F. Grohovaz, E. Brambilla, F. Codazzi, A. Consiglio, E. Clementi, M. Filippi, G. Comi, and L. M. Grimaldi. Proinflammatory cytokines regulate antigen-independent T-cell activation by two separate calcium-signaling pathways in multiple sclerosis patients. Ann. Neurol., 43(3):340-9, mar 1998. 
[244] M. Buntinx, P. Stinissen, P. Steels, M. Ameloot, and J. Raus. Immune-mediated oligodendrocyte injury in multiple sclerosis: molecular mechanisms and therapeutic interventions. Crit. Rev. Immunol., 22(5-6):391-424, jan 2002.

[245] J. K. Fazakerley and R. Walker. Virus demyelination. J. Neurovirol., 9(2):148-64, apr 2003.

[246] D. Baker and S. J. Jackson. Models of Multiple Sclerosis. ACNR, 6(6):4-6, 2007.

[247] H. Wekerle, K. Kojima, J. Lannes-Vieira, H. Lassmann, and C. Linington. Animal models. Ann. Neurol., 36 Suppl:S47-53, jan 1994.

[248] W. F. Blakemore, R. A. Eames, K. J. Smith, and W. I. McDonald. Remyelination in the spinal cord of the cat following intraspinal injections of lysolecithin. J. Neurol. Sci., 33(1-2):31-43, aug 1977.

[249] R. H. Woodruff and R. J. Franklin. Demyelination and remyelination of the caudal cerebellar peduncle of adult rats following stereotaxic injections of lysolecithin, ethidium bromide, and complement/anti-galactocerebroside: a comparative study. Glia, 25(3):216-28, feb 1999.

[250] S. M. Hall. The effect of injections of lysophosphatidyl choline into white matter of the adult mouse spinal cord. J. Cell Sci., 10(2):535-46, mar 1972.

[251] N. D. Jeffery and W. F. Blakemore. Remyelination of mouse spinal cord axons demyelinated by local injection of lysolecithin. J. Neurocytol., 24(10):775-81, oct 1995.

[252] E. Birgbauer, T. S. Rao, and M. Webb. Lysolecithin induces demyelination in vitro in a cerebellar slice culture system. J. Neurosci. Res., 78(2):157-66, oct 2004.

[253] B. V. Bassa, D. D. Roh, N. D. Vaziri, M. A. Kirschenbaum, and V. S. Kamanna. Lysophosphatidylcholine activates mesangial cell PKC and MAP kinase by PLCgamma-1 and tyrosine kinase-Ras pathways. Am. J. Physiol., 277(3 Pt 2):F328-37, sep 1999.

[254] Q. Jing, S. M. Xin, W. B. Zhang, P. Wang, Y. W. Qin, and G. Pei. Lysophosphatidylcholine activates $\mathrm{p} 38$ and p42/44 mitogen-activated protein kinases in monocytic THP-1 cells, but only p38 activation is involved in its stimulated chemotaxis. Circ. Res., 87(1):52-9, jul 2000.

[255] G. K. Matsushima and P. Morell. The neurotoxicant, cuprizone, as a model to study demyelination and remyelination in the central nervous system. Brain Pathol., 11(1):107-16, jan 2001.

[256] L. Xiao, H. Xu, Y. Zhang, Z. Wei, J. He, W. Jiang, X. Li, L. E. Dyck, R. M. Devon, Y. Deng, and X. M. Li. Quetiapine facilitates oligodendrocyte development and prevents mice from myelin breakdown and behavioral changes. Mol. Psychiatry, 13(7):697-708, jul 2008.

[257] G. Venturini. Enzymic activities and sodium, potassium and copper concentrations in mouse brain and liver after cuprizone treatment in vivo. J. Neurochem., 21(5):1147-51, nov 1973.

[258] W. W. Carlton. Studies on the induction of hydrocephalus and spongy degeneration by cuprizone feeding and attempts to antidote the toxicity. Life Sci., 6(1):11-9, jan 1967.

[259] L. A. Pasquini, C. A. Calatayud, A. L. Bertone Uña, V. Millet, J. M. Pasquini, and E. F. Soto. The neurotoxic effect of cuprizone on oligodendrocytes depends on the presence of pro-inflammatory cytokines secreted by microglia. Neurochem. Res., 32(2):279-92, feb 2007.

[260] V. Doan, A. M. Kleindienst, E. J. McMahon, B. R. Long, G. K. Matsushima, and L. C. Taylor. Abbreviated exposure to cuprizone is sufficient to induce demyelination and oligodendrocyte loss. J. Neurosci. Res., 91(3):363-73, mar 2013. 
[261] T. Skripuletz, M. Lindner, A. Kotsiari, N. Garde, J. Fokuhl, F. Linsmeier, C. Trebst, and M. Stangel. Cortical demyelination is prominent in the murine cuprizone model and is strain-dependent. Am. J. Pathol., 172(4):1053-61, apr 2008.

[262] L. C. Taylor, W. Gilmore, and G. K. Matsushima. SJL mice exposed to cuprizone intoxication reveal strain and gender pattern differences in demyelination. Brain Pathol., 19(3):467-79, jul 2009.

[263] K.-A. Irvine and W. F. Blakemore. Age increases axon loss associated with primary demyelination in cuprizone-induced demyelination in C57BL/6 mice. J. Neuroimmunol., 175(1-2):69-76, jun 2006.

[264] A. J. Steelman, J. P. Thompson, and J. Li. Demyelination and remyelination in anatomically distinct regions of the corpus callosum following cuprizone intoxication. Neurosci. Res., 72(1):32-42, jan 2012.

[265] V. Gudi, S. Gingele, T. Skripuletz, and M. Stangel. Glial response during cuprizoneinduced de- and remyelination in the CNS: lessons learned. Front. Cell. Neurosci., 8:73, jan 2014.

[266] P. Morell, C. V. Barrett, J. L. Mason, A. D. Toews, J. D. Hostettler, G. W. Knapp, and G. K. Matsushima. Gene expression in brain during cuprizone-induced demyelination and remyelination. Mol. Cell. Neurosci., 12(4-5):220-7, nov 1998.

[267] S. R. Plant, Y. Wang, S. Vasseur, J. C. Thrash, E. J. McMahon, D. T. Bergstralh, H. A. Arnett, S. D. Miller, M. J. Carson, J. L. Iovanna, and J. P.-Y. Ting. Upregulation of the stress-associated gene $\mathrm{p} 8$ in mouse models of demyelination and in multiple sclerosis tissues. Glia, 53(5):529-37, apr 2006.

[268] W. F. Blakemore. Observations on oligodendrocyte degeneration, the resolution of status spongiosus and remyelination in cuprizone intoxication in mice. J. Neurocytol., 1(4):413-26, dec 1972.

[269] H. Jurevics, C. Largent, J. Hostettler, D. W. Sammond, G. K. Matsushima, A. Kleindienst, A. D. Toews, and P. Morell. Alterations in metabolism and gene expression in brain regions during cuprizone-induced demyelination and remyelination. J. Neurochem., 82(1):126-36, jul 2002.

[270] S. K. Ludwin and E. S. Johnson. Evidence for a "dying-back" gliopathy in demyelinating disease. Ann. Neurol., 9(3):301-5, mar 1981.

[271] K. Suzuki and Y. Kikkawa. Status spongiosus of CNS and hepatic changes induced by cuprizone (biscyclohexanone oxalyldihydrazone). Am. J. Pathol., 54(2):307-25, feb 1969 .

[272] W. F. Blakemore. Demyelination of the superior cerebellar peduncle in the mouse induced by cuprizone. J. Neurol. Sci., 20(1):63-72, sep 1973.

[273] J. D. Thiessen, Y. Zhang, H. Zhang, L. Wang, R. Buist, M. R. Del Bigio, J. Kong, X.-M. Li, and M. Martin. Quantitative MRI and ultrastructural examination of the cuprizone mouse model of demyelination. NMR Biomed., 26(11):1562-81, nov 2013.

[274] F. Mokhtarian, D. E. McFarlin, and C. S. Raine. Adoptive transfer of myelin basic protein-sensitized $\mathrm{T}$ cells produces chronic relapsing demyelinating disease in mice. Nature, 309(5966):356-8, jan 1984.

[275] U. Traugott, C. S. Raine, and D. E. McFarlin. Acute experimental allergic encephalomyelitis in the mouse: immunopathology of the developing lesion. Cell. Immunol., 91(1):240-54, mar 1985.

[276] I. Huitinga, N. van Rooijen, C. J. de Groot, B. M. Uitdehaag, and C. D. Dijkstra. Suppression of experimental allergic encephalomyelitis in Lewis rats after elimination of macrophages. J. Exp. Med., 172(4):1025-33, oct 1990. 
[277] H. Wekerle, B. Engelhardt, W. Risau, and R. Meyermann. Interaction of T lymphocytes with cerebral endothelial cells in vitro. Brain Pathol., 1(2):107-14, jan 1991.

[278] W. F. Hickey, B. L. Hsu, and H. Kimura. T-lymphocyte entry into the central nervous system. J. Neurosci. Res., 28(2):254-60, feb 1991.

[279] S. S. Zamvil and L. Steinman. The T lymphocyte in experimental allergic encephalomyelitis. Annu. Rev. Immunol., 8:579-621, jan 1990.

[280] T. Nomura, Y. Bando, H. Bochimoto, D. Koga, T. Watanabe, and S. Yoshida. Three-dimensional ultra-structures of myelin and the axons in the spinal cord: application of SEM with the osmium maceration method to the central nervous system in two mouse models. Neurosci. Res., 75(3):190-7, mar 2013.

[281] R. M. Ransohoff. Animal models of multiple sclerosis: the good, the bad and the bottom line. Nat. Neurosci., 15(8):1074-7, aug 2012.

[282] D. Baker and S. J. Jackson. No Title. ACNR, 6(6):10-12, 2007.

[283] D. Baker, J. K. O’Neill, S. E. Gschmeissner, C. E. Wilcox, C. Butter, and J. L. Turk. Induction of chronic relapsing experimental allergic encephalomyelitis in Biozzi mice. J. Neuroimmunol., 28(3):261-70, aug 1990.

[284] J. K. O'Neill, D. Baker, and J. L. Turk. Inhibition of chronic relapsing experimental allergic encephalomyelitis in the Biozzi AB/H mouse. J. Neuroimmunol., 41(2):17787, dec 1992.

[285] N. M. Thoua, J. M. van Noort, D. Baker, A. Bose, A. C. van Sechel, M. J. van Stipdonk, P. J. Travers, and S. Amor. Encephalitogenic and immunogenic potential of the stress protein alphaB-crystallin in Biozzi ABH $(\mathrm{H}-2 \mathrm{~A}(\mathrm{~g} 7))$ mice. J. Neuroimmunol., 104(1):47-57, apr 2000.

[286] S. Amor, N. Groome, C. Linington, M. M. Morris, K. Dornmair, M. V. Gardinier, J. M. Matthieu, and D. Baker. Identification of epitopes of myelin oligodendrocyte glycoprotein for the induction of experimental allergic encephalomyelitis in SJL and Biozzi AB/H mice. J. Immunol., 153(10):4349-56, nov 1994.

[287] J. K. O’Neill, D. Baker, M. M. Morris, S. E. Gschmeissner, H. G. Jenkins, A. M. Butt, S. L. Kirvell, and S. Amor. Optic neuritis in chronic relapsing experimental allergic encephalomyelitis in Biozzi ABH mice: demyelination and fast axonal transport changes in disease. J. Neuroimmunol., 82(2):210-8, mar 1998.

[288] J. Trotter and M. E. Smith. The role of phospholipases from inflammatory macrophages in demyelination. Neurochem. Res., 11(3):349-61, mar 1986.

[289] P. Z. Goldenberg, E. E. Kwon, J. A. Benjamins, J. N. Whitaker, R. H. Quarles, and J. W. Prineas. Opsonization of normal myelin by anti-myelin antibodies and normal serum. J. Neuroimmunol., 23(2):157-66, jul 1989.

[290] G. R. Moore and C. S. Raine. Immunogold localization and analysis of IgG during immune-mediated demyelination. Lab. Invest., 59(5):641-8, nov 1988.

[291] H. J. Schluesener, R. A. Sobel, C. Linington, and H. L. Weiner. A monoclonal antibody against a myelin oligodendrocyte glycoprotein induces relapses and demyelination in central nervous system autoimmune disease. J. Immunol., 139(12):4016-21, dec 1987 .

[292] C. Linington, M. Bradl, H. Lassmann, C. Brunner, and K. Vass. Augmentation of demyelination in rat acute allergic encephalomyelitis by circulating mouse monoclonal antibodies directed against a myelin/oligodendrocyte glycoprotein. Am. J. Pathol., 130(3):443-54, mar 1988.

[293] D. M. Wingerchuk. Neuromyelitis optica: new findings on pathogenesis. Int. Rev. Neurobiol., 79:665-88, jan 2007. 
[294] W. Brück, B. Popescu, C. F. Lucchinetti, S. Markovic-Plese, R. Gold, D. R. Thal, and I. Metz. Neuromyelitis optica lesions may inform multiple sclerosis heterogeneity debate. Ann. Neurol., 72(3):385-94, sep 2012.

[295] J. D. E. Parratt and J. W. Prineas. Neuromyelitis optica: a demyelinating disease characterized by acute destruction and regeneration of perivascular astrocytes. Mult. Scler., 16(10):1156-72, oct 2010.

[296] C. F. Lucchinetti, R. N. Mandler, D. McGavern, W. Bruck, G. Gleich, R. M. Ransohoff, C. Trebst, B. Weinshenker, D. Wingerchuk, J. E. Parisi, and H. Lassmann. A role for humoral mechanisms in the pathogenesis of Devic's neuromyelitis optica. Brain, 125(Pt 7):1450-61, jul 2002.

[297] T. Misu, K. Fujihara, A. Kakita, H. Konno, M. Nakamura, S. Watanabe, T. Takahashi, I. Nakashima, H. Takahashi, and Y. Itoyama. Loss of aquaporin 4 in lesions of neuromyelitis optica: distinction from multiple sclerosis. Brain, 130(Pt 5):1224-34, may 2007.

[298] C. Papeix, J.-S. Vidal, J. de Seze, C. Pierrot-Deseilligny, A. Tourbah, B. Stankoff, C. Lebrun, T. Moreau, P. Vermersch, B. Fontaine, O. Lyon-Caen, and O. Gout. Immunosuppressive therapy is more effective than interferon in neuromyelitis optica. Mult. Scler., 13(2):256-9, mar 2007.

[299] V. A. Lennon, D. M. Wingerchuk, T. J. Kryzer, S. J. Pittock, C. F. Lucchinetti, K. Fujihara, I. Nakashima, and B. G. Weinshenker. A serum autoantibody marker of neuromyelitis optica: distinction from multiple sclerosis. Lancet, 364(9451):2106-12, jan.

[300] V. A. Lennon, T. J. Kryzer, S. J. Pittock, A. S. Verkman, and S. R. Hinson. IgG marker of optic-spinal multiple sclerosis binds to the aquaporin- 4 water channel. $J$. Exp. Med., 202(4):473-7, aug 2005.

[301] J. L. Bennett, C. Lam, S. R. Kalluri, P. Saikali, K. Bautista, C. Dupree, M. Glogowska, D. Case, J. P. Antel, G. P. Owens, D. Gilden, S. Nessler, C. Stadelmann, and B. Hemmer. Intrathecal pathogenic anti-aquaporin-4 antibodies in early neuromyelitis optica. Ann. Neurol., 66(5):617-29, nov 2009.

[302] T. Takahashi, K. Fujihara, I. Nakashima, T. Misu, I. Miyazawa, M. Nakamura, S. Watanabe, Y. Shiga, C. Kanaoka, J. Fujimori, S. Sato, and Y. Itoyama. Antiaquaporin-4 antibody is involved in the pathogenesis of NMO: a study on antibody titre. Brain, 130(Pt 5):1235-43, may 2007.

[303] S. Jarius, F. Aboul-Enein, P. Waters, B. Kuenz, A. Hauser, T. Berger, W. Lang, M. Reindl, A. Vincent, and W. Kristoferitsch. Antibody to aquaporin-4 in the long-term course of neuromyelitis optica. Brain, 131(Pt 11):3072-80, nov 2008.

[304] S. F. Roemer, J. E. Parisi, V. A. Lennon, E. E. Benarroch, H. Lassmann, W. Bruck, R. N. Mandler, B. G. Weinshenker, S. J. Pittock, D. M. Wingerchuk, and C. F. Lucchinetti. Pattern-specific loss of aquaporin-4 immunoreactivity distinguishes neuromyelitis optica from multiple sclerosis. Brain, 130(Pt 5):1194-205, may 2007.

[305] H. Zhang, J. L. Bennett, and A. S. Verkman. Ex vivo spinal cord slice model of neuromyelitis optica reveals novel immunopathogenic mechanisms. Ann. Neurol., 70(6):943-54, dec 2011.

[306] M. Bradl, T. Misu, T. Takahashi, M. Watanabe, S. Mader, M. Reindl, M. Adzemovic, J. Bauer, T. Berger, K. Fujihara, Y. Itoyama, and H. Lassmann. Neuromyelitis optica: pathogenicity of patient immunoglobulin in vivo. Ann. Neurol., 66(5):630-43, nov 2009.

[307] C. Wrzos, A. Winkler, I. Metz, D. M. Kayser, D. R. Thal, C. Wegner, W. Brück, S. Nessler, J. L. Bennett, and C. Stadelmann. Early loss of oligodendrocytes in human and experimental neuromyelitis optica lesions. Acta Neuropathol., 127(4):52338 , apr 2014. 
[308] M. Kinoshita, Y. Nakatsuji, T. Kimura, M. Moriya, K. Takata, T. Okuno, A. Kumanogoh, K. Kajiyama, H. Yoshikawa, and S. Sakoda. Anti-aquaporin-4 antibody induces astrocytic cytotoxicity in the absence of CNS antigen-specific T cells. Biochem. Biophys. Res. Commun., 394(1):205-10, mar 2010.

[309] S. Saadoun, P. Waters, B. A. Bell, A. Vincent, A. S. Verkman, and M. C. Papadopoulos. Intra-cerebral injection of neuromyelitis optica immunoglobulin $\mathrm{G}$ and human complement produces neuromyelitis optica lesions in mice. Brain, 133(Pt 2):349-61, feb 2010.

[310] M. Kinoshita, Y. Nakatsuji, T. Kimura, M. Moriya, K. Takata, T. Okuno, A. Kumanogoh, K. Kajiyama, H. Yoshikawa, and S. Sakoda. Neuromyelitis optica: Passive transfer to rats by human immunoglobulin. Biochem. Biophys. Res. Commun., 386(4):623-7, sep 2009.

[311] R. Marignier, A. Nicolle, C. Watrin, M. Touret, S. Cavagna, M. Varrin-Doyer, G. Cavillon, V. Rogemond, C. Confavreux, J. Honnorat, and P. Giraudon. Oligodendrocytes are damaged by neuromyelitis optica immunoglobulin $\mathrm{G}$ via astrocyte injury. Brain, 133(9):2578-91, sep 2010.

[312] S. R. Hinson, M. F. Romero, B. F. G. Popescu, C. F. Lucchinetti, J. P. Fryer, H. Wolburg, P. Fallier-Becker, S. Noell, and V. A. Lennon. Molecular outcomes of neuromyelitis optica (NMO)-IgG binding to aquaporin-4 in astrocytes. Proc. Natl. Acad. Sci. U. S. A., 109(4):1245-50, jan 2012.

[313] Y. Zhou and N. C. Danbolt. Glutamate as a neurotransmitter in the healthy brain. J. Neural Transm., 121(8):799-817, aug 2014.

[314] S. R. Hinson, S. F. Roemer, C. F. Lucchinetti, J. P. Fryer, T. J. Kryzer, J. L. Chamberlain, C. L. Howe, S. J. Pittock, and V. A. Lennon. Aquaporin-4-binding autoantibodies in patients with neuromyelitis optica impair glutamate transport by down-regulating EAAT2. J. Exp. Med., 205(11):2473-81, oct 2008.

[315] J. Ratelade, N. Asavapanumas, A. M. Ritchie, S. Wemlinger, J. L. Bennett, and A. S. Verkman. Involvement of antibody-dependent cell-mediated cytotoxicity in inflammatory demyelination in a mouse model of neuromyelitis optica. Acta Neuropathol., 126(5):699-709, nov 2013.

[316] P.-W. Phuan, H. Zhang, N. Asavapanumas, M. Leviten, A. Rosenthal, L. Tradtrantip, and A. S. Verkman. C1q-targeted monoclonal antibody prevents complement-dependent cytotoxicity and neuropathology in in vitro and mouse models of neuromyelitis optica. Acta Neuropathol., 125(6):829-40, jun 2013.

[317] G. P. Nicchia, M. Mastrototaro, A. Rossi, F. Pisani, C. Tortorella, M. Ruggieri, A. Lia, M. Trojano, A. Frigeri, and M. Svelto. Aquaporin-4 orthogonal arrays of particles are the target for neuromyelitis optica autoantibodies. Glia, 57(13):136373 , oct 2009 .

[318] K. Ikeda, N. Kiyota, H. Kuroda, D. K. Sato, S. Nishiyama, T. Takahashi, T. Misu, I. Nakashima, K. Fujihara, and M. Aoki. Severe demyelination but no astrocytopathy in clinically definite neuromyelitis optica with anti-myelin-oligodendrocyte glycoprotein antibody. Mult. Scler., 21(5):656-9, apr 2015.

[319] M. C. Papadopoulos, J. L. Bennett, and A. S. Verkman. Treatment of neuromyelitis optica: state-of-the-art and emerging therapies. Nat. Rev. Neurol., 10(9):493-506, sep 2014.

[320] S. Kuhlmann-Krieg, I. Sommer, and M. Schachner. Ultrastructural features of cultured oligodendrocytes expressing stage-specific cell-surface antigens. Brain Res., 467(2):269-80, apr 1988.

[321] M. C. Raff, R. H. Miller, and M. Noble. A glial progenitor cell that develops in vitro into an astrocyte or an oligodendrocyte depending on culture medium. Nature, 303(5916):390-396, jun 1983. 
[322] J. Trotter and M. Schachner. Cells positive for the O4 surface antigen isolated by cell sorting are able to differentiate into astrocytes or oligodendrocytes. Brain Res. Dev. Brain Res., 46(1):115-22, mar 1989.

[323] K. Trajkovic, A. S. Dhaunchak, J. T. Goncalves, D. Wenzel, A. Schneider, G. Bunt, K.-A. Nave, and M. Simons. Neuron to glia signaling triggers myelin membrane exocytosis from endosomal storage sites. J. Cell Biol., 172(6):937-48, mar 2006.

[324] K. V. Toyka, D. B. Brachman, A. Pestronk, and I. Kao. Myasthenia gravis: passive transfer from man to mouse. Science, 190(4212):397-9, oct 1975.

[325] H. Kluver and E. Barrera. A method for the combined staining of cells and fibers in the nervous system. J. Neuropathol. Exp. Neurol., 12(4):400-3, oct 1953.

[326] N. B. Eisele, S. Frey, J. Piehler, D. Görlich, and R. P. Richter. Ultrathin nucleoporin phenylalanine-glycine repeat films and their interaction with nuclear transport receptors. EMBO Rep., 11(5):366-72, may 2010.

[327] N. Kahya, E. I. Pécheur, W. P. de Boeij, D. A. Wiersma, and D. Hoekstra. Reconstitution of membrane proteins into giant unilamellar vesicles via peptide-induced fusion. Biophys. J., 81(3):1464-74, sep 2001.

[328] S. Ruskamo, M. Chukhlieb, J. Vahokoski, S. P. Bhargav, F. Liang, I. Kursula, and P. Kursula. Juxtanodin is an intrinsically disordered F-actin-binding protein. Sci. Rep., 2:899, jan 2012.

[329] S. Ruskamo, R. P. Yadav, S. Sharma, M. Lehtimäki, S. Laulumaa, S. Aggarwal, M. Simons, J. Bürck, A. S. Ulrich, A. H. Juffer, I. Kursula, and P. Kursula. Atomic resolution view into the structure-function relationships of the human myelin peripheral membrane protein P2. Acta Crystallogr. D. Biol. Crystallogr., 70(Pt 1):16576, jan 2014.

[330] G. Muruganandam, A. S. Ulrich, I. Kursula, and P. Kursula. Lipid Membrane Association of Myelin Proteins and Peptide Segments Studied by Oriented and Synchrotron Radiation Circular Dichroism Spectroscopy. 2013.

[331] W. Liou, H. J. Geuze, and J. W. Slot. Improving structural integrity of cryosections for immunogold labeling. Histochem. Cell Biol., 106(1):41-58, jul 1996.

[332] W. Möbius, B. Cooper, W. A. Kaufmann, C. Imig, T. Ruhwedel, N. Snaidero, A. S. Saab, and F. Varoqueaux. Electron microscopy of the mouse central nervous system. Methods Cell Biol., 96:475-512, jan 2010.

[333] H. B. Werner, K. Kuhlmann, S. Shen, M. Uecker, A. Schardt, K. Dimova, F. Orfaniotou, A. Dhaunchak, B. G. Brinkmann, W. Möbius, L. Guarente, P. CasacciaBonnefil, O. Jahn, and K.-A. Nave. Proteolipid protein is required for transport of sirtuin 2 into CNS myelin. J. Neurosci., 27(29):7717-30, jul 2007.

[334] P. J. Peters and J. Pierson. Immunogold labeling of thawed cryosections. Methods Cell Biol., 88:131-49, jan 2008.

[335] M. Jung, I. Sommer, M. Schachner, and K. A. Nave. Monoclonal antibody O10 defines a conformationally sensitive cell-surface epitope of proteolipid protein (PLP): evidence that PLP misfolding underlies dysmyelination in mutant mice. J. Neurosci., 16(24):7920-9, dec 1996.

[336] N. Korogod, C. C. Petersen, and G. W. Knott. Ultrastructural analysis of adult mouse neocortex comparing aldehyde perfusion with cryo fixation. Elife, 4:e05793, aug 2015.

[337] Y. Itoyama and H. D. Webster. Immunocytochemical study of myelin-associated glycoprotein (MAG) and basic protein (BP) in acute experimental allergic encephalomyelitis (EAE). J. Neuroimmunol., 3(4):351-64, dec 1982. 
[338] A. K. Pröbstel, K. Dornmair, R. Bittner, P. Sperl, D. Jenne, S. Magalhaes, A. Villalobos, C. Breithaupt, R. Weissert, U. Jacob, M. Krumbholz, T. Kuempfel, A. Blaschek, W. Stark, J. Gärtner, D. Pohl, K. Rostasy, F. Weber, I. Forne, M. Khademi, T. Olsson, F. Brilot, E. Tantsis, R. C. Dale, H. Wekerle, R. Hohlfeld, B. Banwell, A. Bar-Or, E. Meinl, and T. Derfuss. Antibodies to MOG are transient in childhood acute disseminated encephalomyelitis. Neurology, 77(6):580-8, aug 2011.

[339] D. K. Sato, D. Callegaro, M. A. Lana-Peixoto, P. J. Waters, F. M. de Haidar Jorge, T. Takahashi, I. Nakashima, S. L. Apostolos-Pereira, N. Talim, R. F. Simm, A. M. M. Lino, T. Misu, M. I. Leite, M. Aoki, and K. Fujihara. Distinction between MOG antibody-positive and AQP4 antibody-positive NMO spectrum disorders. Neurology, 82(6):474-81, feb 2014.

[340] S. Kuenzle, H.-C. von Büdingen, M. Meier, M. D. Harrer, E. Urich, B. Becher, and N. Goebels. Pathogen specificity and autoimmunity are distinct features of antigen-driven immune responses in neuroborreliosis. Infect. Immun., 75(8):3842-7, aug 2007.

[341] M. M. Morris, S. Piddlesden, N. Groome, and S. Amor. Anti-myelin antibodies modulate experimental allergic encephalomyelitis in Biozzi ABH mice. Biochem. Soc. Trans., 25(2):168S, may 1997.

[342] S. Al-Izki, G. Pryce, J. K. O'Neill, C. Butter, G. Giovannoni, S. Amor, and D. Baker. Practical guide to the induction of relapsing progressive experimental autoimmune encephalomyelitis in the Biozzi ABH mouse. Mult. Scler. Relat. Disord., 1(1):29-38, jan 2012 .

[343] P. Li, S. Banjade, H.-C. Cheng, S. Kim, B. Chen, L. Guo, M. Llaguno, J. V. Hollingsworth, D. S. King, S. F. Banani, P. S. Russo, Q.-X. Jiang, B. T. Nixon, and M. K. Rosen. Phase transitions in the assembly of multivalent signalling proteins. Nature, 483(7389):336-40, mar 2012.

[344] S. Frey and D. Görlich. A saturated FG-repeat hydrogel can reproduce the permeability properties of nuclear pore complexes. Cell, 130(3):512-23, aug 2007.

[345] S. Frey, R. P. Richter, and D. Görlich. FG-rich repeats of nuclear pore proteins form a three-dimensional meshwork with hydrogel-like properties. Science, 314(5800):815-7, nov 2006.

[346] B. Kachar, T. Behar, and M. Dubois-Dalcq. Cell shape and motility of oligodendrocytes cultured without neurons. Cell Tissue Res., 244(1):27-38, jan 1986.

[347] A. Matsuo, I. Akiguchi, G. C. Lee, E. G. McGeer, P. L. McGeer, and J. Kimura. Myelin degeneration in multiple system atrophy detected by unique antibodies. $\mathrm{Am}$. J. Pathol., 153(3):735-44, sep 1998.

[348] S. Nawaz, A. Kippert, A. S. Saab, H. B. Werner, T. Lang, K.-A. Nave, and M. Simons. Phosphatidylinositol 4,5-bisphosphate-dependent interaction of myelin basic protein with the plasma membrane in oligodendroglial cells and its rapid perturbation by elevated calcium. J. Neurosci., 29(15):4794-807, apr 2009.

[349] S. K. Rodal, G. Skretting, O. Garred, F. Vilhardt, B. van Deurs, and K. Sandvig. Extraction of Cholesterol with Methyl-beta -Cyclodextrin Perturbs Formation of Clathrin-coated Endocytic Vesicles. Mol. Biol. Cell, 10(4):961-974, apr 1999.

[350] C. A. Dyer and J. A. Benjamins. Antibody to galactocerebroside alters organization of oligodendroglial membrane sheets in culture. J. Neurosci., 8(11):4307-18, nov 1988.

[351] W. F. Blakemore. Remyelination of the superior cerebellar peduncle in the mouse following demyelination induced by feeding cuprizone. J. Neurol. Sci., 20(1):73-83, sep 1973. 
[352] L.-J. Oluich, J. A. S. Stratton, Y. L. Xing, S. W. Ng, H. S. Cate, P. Sah, F. Windels, T. J. Kilpatrick, and T. D. Merson. Targeted ablation of oligodendrocytes induces axonal pathology independent of overt demyelination. J. Neurosci., 32(24):8317-30, jun 2012 .

[353] K.-A. Nave and H. B. Werner. Myelination of the Nervous System: Mechanisms and Functions. Annu. Rev. Cell Dev. Biol., 30(1):503-533, oct 2014.

[354] J. E. Rash. Molecular disruptions of the panglial syncytium block potassium siphoning and axonal saltatory conduction: pertinence to neuromyelitis optica and other demyelinating diseases of the central nervous system. Neuroscience, 168(4):9821008, jul 2010.

[355] Y. Fu, H. Wang, T. B. Huff, R. Shi, and J.-X. Cheng. Coherent anti-Stokes Raman scattering imaging of myelin degradation reveals a calcium-dependent pathway in lyso-PtdCho-induced demyelination. J. Neurosci. Res., 85(13):2870-81, oct 2007.

[356] M. Rodriguez, B. W. Scheithauer, G. Forbes, and P. J. Kelly. Oligodendrocyte injury is an early event in lesions of multiple sclerosis. Mayo Clin. Proc., 68(7):627-36, jul 1993.

[357] K. Suzuki and K. Suzuki. Myelin Pathology in the Twitcher Mouse. Ann. N. Y. Acad. Sci., 605(1 Myelination a):313-324, nov 1990.

[358] S. M. Hall and N. A. Gregson. The in vivo and ultrastructural effects of injection of lysophosphatidyl choline into myelinated peripheral nerve fibres of the adult mouse. J. Cell Sci., 9(3):769-89, nov 1971.

[359] W. W. Schlaepfer. Vesicular disruption of myelin simulated by exposure of nerve to calcium ionophore. Nature, 265(5596):734-6, feb 1977.

[360] K. J. Smith, S. M. Hall, and C. L. Schauf. Vesicular demyelination induced by raised intracellular calcium. J. Neurol. Sci., 71(1):19-37, nov 1985.

[361] T. Saida, K. Saida, M. J. Brown, and D. H. Silberberg. Peripheral nerve demyelination induced by intraneural injection of experimental allergic encephalomyelitis serum. J. Neuropathol. Exp. Neurol., 38(5):498-518, sep 1979.

[362] A. F. Hahn, J. J. Gilbert, and T. E. Feasby. Passive transfer of demyelination by experimental allergic neuritis serum. Acta Neuropathol., 49(3):169-76, jan 1980.

[363] M. C. Dal Canto and H. L. Lipton. Primary demyelination in Theiler's virus infection. An ultrastructural study. Lab. Invest., 33(6):626-37, dec 1975.

[364] J. D. Balentine. Pathology of experimental spinal cord trauma. II. Ultrastructure of axons and myelin. Lab. Invest., 39(3):254-66, sep 1978.

[365] C. S. Raine, B. Cannella, S. L. Hauser, and C. P. Genain. Demyelination in primate autoimmune encephalomyelitis and acute multiple sclerosis lesions: a case for antigen-specific antibody mediation. Ann. Neurol., 46(2):144-60, aug 1999.

[366] C. P. Genain, B. Cannella, S. L. Hauser, and C. S. Raine. Identification of autoantibodies associated with myelin damage in multiple sclerosis. Nat. Med., 5(2):170-5, feb 1999 .

[367] R. Yamasaki, H. Lu, O. Butovsky, N. Ohno, A. M. Rietsch, R. Cialic, P. M. Wu, C. E. Doykan, J. Lin, A. C. Cotleur, G. Kidd, M. M. Zorlu, N. Sun, W. Hu, L. Liu, J.-C. Lee, S. E. Taylor, L. Uehlein, D. Dixon, J. Gu, C. M. Floruta, M. Zhu, I. F. Charo, H. L. Weiner, and R. M. Ransohoff. Differential roles of microglia and monocytes in the inflamed central nervous system. J. Exp. Med., 211(8):1533-49, jul 2014.

[368] W. Möbius, J. Patzig, K.-A. Nave, and H. B. Werner. Phylogeny of proteolipid proteins: divergence, constraints, and the evolution of novel functions in myelination and neuroprotection. Neuron Glia Biol., 4(2):111-27, may 2008. 
[369] M. Simons, T. Misgeld, and M. Kerschensteiner. A unified cell biological perspective on axon-myelin injury. J. Cell Biol., 206(3):335-345, aug 2014.

[370] G. S. T. Smith, P. M. Paez, V. Spreuer, C. W. Campagnoni, J. M. Boggs, A. T. Campagnoni, and G. Harauz. Classical 18.5-and 21.5-kDa isoforms of myelin basic protein inhibit calcium influx into oligodendroglial cells, in contrast to golli isoforms. J. Neurosci. Res., 89(4):467-80, apr 2011.

[371] C. A. Dyer. Novel oligodendrocyte transmembrane signaling systems. Investigations utilizing antibodies as ligands. Mol. Neurobiol., 7(1):1-22, jan 1993.

[372] P. K. Stys. White matter injury mechanisms. Curr. Mol. Med., 4(2):113-30, mar 2004.

[373] I. Micu, Q. Jiang, E. Coderre, A. Ridsdale, L. Zhang, J. Woulfe, X. Yin, B. D. Trapp, J. E. McRory, R. Rehak, G. W. Zamponi, W. Wang, and P. K. Stys. NMDA receptors mediate calcium accumulation in myelin during chemical ischaemia. Nature, 439(7079):988-92, mar 2006.

[374] F. G. Mastronardi and M. A. Moscarello. Molecules affecting myelin stability: a novel hypothesis regarding the pathogenesis of multiple sclerosis. J. Neurosci. Res., 80(3):301-8, may 2005.

[375] M. A. Moscarello, G. W. Brady, D. B. Fein, D. D. Wood, and T. F. Cruz. The role of charge microheterogeneity of basic protein in the formation and maintenance of the multilayered structure of myelin: a possible role in multiple sclerosis. J. Neurosci. Res., 15(1):87-99, jan 1986.

[376] N. L. Banik, W. W. McAlhaney, and E. L. Hogan. Calcium-stimulated proteolysis in myelin: evidence for a Ca2+-activated neutral proteinase associated with purified myelin of rat CNS. J. Neurochem., 45(2):581-8, aug 1985.

[377] N. L. Banik. Pathogenesis of myelin breakdown in demyelinating diseases: role of proteolytic enzymes. Crit. Rev. Neurobiol., 6(4):257-71, jan 1992.

[378] B. Ohler, K. Graf, R. Bragg, T. Lemons, R. Coe, C. Genain, J. Israelachvili, and C. Husted. Role of lipid interactions in autoimmune demyelination. Biochim. Biophys. Acta, 1688(1):10-7, jan 2004.

[379] W. Cammer, B. R. Bloom, W. T. Norton, and S. Gordon. Degradation of basic protein in myelin by neutral proteases secreted by stimulated macrophages: a possible mechanism of inflammatory demyelination. Proc. Natl. Acad. Sci. U. S. A., 75(3):1554-8, mar 1978.

[380] D. A. Compston, B. P. Morgan, D. Oleesky, R. Fifield, and A. K. Campbell. Cerebrospinal fluid C9 in demyelinating disease. Neurology, 36(11):1503-6, nov 1986.

[381] D. A. Compston and N. J. Scolding. Immune-mediated oligodendrocyte injury. Ann. N. Y. Acad. Sci., 633:196-204, jan 1991.

[382] M. Kato, T. W. Han, S. Xie, K. Shi, X. Du, L. C. Wu, H. Mirzaei, E. J. Goldsmith, J. Longgood, J. Pei, N. V. Grishin, D. E. Frantz, J. W. Schneider, S. Chen, L. Li, M. R. Sawaya, D. Eisenberg, R. Tycko, and S. L. McKnight. Cell-free formation of RNA granules: low complexity sequence domains form dynamic fibers within hydrogels. Cell, 149(4):753-67, may 2012.

[383] A. A. Hyman, C. A. Weber, and F. Jülicher. Liquid-liquid phase separation in biology. Annu. Rev. Cell Dev. Biol., 30:39-58, jan 2014.

[384] V. N. Uversky. The triple power of D: protein intrinsic disorder in degenerative diseases. Front. Biosci. (Landmark Ed., 19:181-258, jan 2014.

[385] C. Richter-Landsberg. Protein Aggregate Formation In Oligodendrocytes: Tau And The Cytoskeleton At The Intersection Of Neuroprotection And Neurodegeneration. Biol. Chem., jun 2015. 
[386] J. H. Wong, G. M. Halliday, and W. S. Kim. Exploring myelin dysfunction in multiple system atrophy. Exp. Neurobiol., 23(4):337-44, dec 2014.

[387] A. Matsuo, G. C. Lee, K. Terai, K. Takami, W. F. Hickey, E. G. McGeer, and P. L. McGeer. Unmasking of an unusual myelin basic protein epitope during the process of myelin degeneration in humans: a potential mechanism for the generation of autoantigens. Am. J. Pathol., 150(4):1253-66, apr 1997. 



\section{Curriculum Vitae}

Marie-Theres Weil

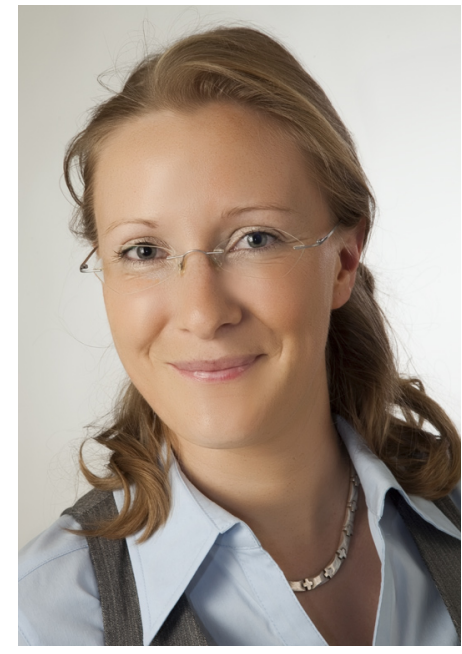

born on $22^{\text {th }}$ July 1987

in Mainz, Germany

Immanuel-Kant-Str. 28

37083 Göttingen, Germany

Phone +49 (151)-46520019

E-Mail weil@em.mpg.de

\section{Education}

02/2012 - present PhD thesis: Molecular mechanisms of myelin disassembly, Max Planck Institute for Experimental Medicine, Göttingen, Germany

03/2010-10/2011 Master of Science in Biomedical Sciences and Technology, University of Applied Sciences in Mannheim, Germany

10/2006-02/2010 Bachelor of Science in Biological Chemistry, University of Applied Sciences in Mannheim, Germany Secondary School (Gymnasium in Nieder-Olm, Germany) 


\section{Employment}

$09-12 / 2011$

03/2011-09/2011

03/2009-07/2009

$10 / 2006-02 / 2010$

$10 / 2009-01 / 2010$

08/2008-01/2009
Research Assistant at the University of Applied Sciences Mannheim in the Institute of Analytical Chemistry

Master thesis at pharmaceutical company Abbott in the Laboratory "GGRP Pharmacology", Ludwigshafen, Germany

Research Assistant at the University of Applied Sciences Mannheim in the Institute of Analytical Chemistry

Student research project at the University of Applied Sciences Mannheim in the "Molecular Cell Biology Laboratory"

Bachelor thesis at the "Alzheimer Cell Biology Laboratory" in Sydney, Australia

Internship at the Kerry Packer Research Institute in Westmead in the "Genetic Metabolic Diseases Laboratory", Sydney, Australia

\section{Scholarships \& Prizes}

\section{Scientific Talks}

Award by the University of Applied Sciences Mannheim as best graduating student of the master course

Award by the University of Applied Sciences Mannheim as best graduating student of the bachelor course

DAAD-scholarship "Temporary employment abroad for the completion of degree work" Scholarship from the University of Applied Sciences Mannheim as one of the two best students in faculty
Microscopy Conference in Göttingen, Germany Symposium "Current Topics in Myelin Research" in Kassel, Germany Joint lab symposium "Cellular Mechanisms of Nervous System Development and Disease" in Munich, Germany

Joint lab symposium "Cell Biology of Myelinating Glia" in Göttingen, Germany Forschungskonferenz der Universitätsmedizin Göttingen, Germany 


\section{Posters}

2015 Marie-Theres Weil, Wiebke Möbius, Torben Ruhwedel, Steffen Frey, Petri Kursula, Martin Kerschensteiner, Christine StadelmannNessler, Mikael Simons, "Molecular mechanisms of myelin disassembly", Conference XII European Meeting on Glial Cells in Health and Disease, Bilbao, Spain

2015 Marie-Theres Weil, Wiebke Möbius, Torben Ruhwedel, Steffen Frey, Petri Kursula, Martin Kerschensteiner, Christine StadelmannNessler, Mikael Simons, "Molecular mechanisms of myelin disassembly", SAB meeting, Göttingen, Germany

2014 Marie-Theres Weil, Anne Flach, Fred Lühder, Gesa Pähler, Mikael Simons, "Distortion of the membrane binding of MBP causes its structural change and leads to decompaction in vitro", PhD retreat, Steinhudder Meer, Germany

2014 Marie-Theres Weil, Anne Flach, Fred Lühder, Gesa Pähler, Mikael Simons, "Distortion of the membrane binding of MBP causes its structural change and leads to decompaction in vitro", GGNB Science day, Göttingen, Germany

2013 Marie-Theres Weil, S. Löblein, J. Lochead, T. Werner "Kontaktloses Glukose-Monitoring in 24-well Zellkulturplatten", ideas competition Biotechnology and medical technology, Baden-Württemberg, Stuttgart, Germany

\section{Publications}

Weil MT., Möbius M., Winkler A., Ruhwedel T., Raasakka A., Bennett J., Enz L., Frey S., Kursula P., Schaeren-Wiemers N., Stadelmann-Nessler C., Simons M. (2015). A phase transition of myelin basic proteins triggers myelin breakdown during demyelination, submitted

Aggarwal S1., Snaidero N., Pähler G., Frey S., Sanchez P., Zweckstetter M., Janshoff A., Schneider A., Weil MT., Schaap IA., Görlich D., Simons M. (2013). Myelin membrane assembly is driven by a phase transition of myelin basic proteins into a cohesive protein meshwork, PLOS Biol, 11(6)

Weil, MT. and Werner, T. (2012) High Throughput Respiration Assay for Cytotoxicity Determination - Development of a New and Time Saving Method for Evaluation of New Active Pharmaceutical Ingredients, GIT Laboratory

Journal Europe 


\section{Languages}

Mother tongue: German

other languages: fluent english, good french

\section{Soft skills}

PhD representative from 2013 to 2014 (involved organization of monthly $\mathrm{PhD}$ student meetings and the annual retreat),

Supervision of students during master practical course Assay Development, during $\mathrm{PhD}$ (one master student, one lab rotation student) and one student research project (for 2 months),

Participation in the student council (semester 2008 /2009),

Mentoring a soccer team with disabled persons,

Volunteer work with youth groups (1.MBC Speyer),

Training as Mediator.

\section{Technical skills}

Good computer knowledge (Windows, Excel, Word, Powerpoint, Origin, GraphPad Prism, Abode Photoshop and Illustrator, ImageJ, several Reference Manager etc.) 